\title{
Dynamics of Deforming Drops
}

Wilco Bouwhuis 
DYNAMICS OF DEFORMING DROPS

Wilco Bouwhuis 


\section{Samenstelling promotiecommissie:}

Prof. dr. Hans Hilgenkamp (voorzitter) Universiteit Twente

Prof. dr. Jacco H. Snoeijer (promotor) Universiteit Twente

Prof. dr. Devaraj van der Meer (promotor) Universiteit Twente

Prof. dr. Philippe Brunet

University of Paris Diderot,

France

Prof. dr. Michiel T. Kreutzer

Ir. Michel Riepen

Technische Universiteit Delft

ASML

Prof. dr. Serge G. Lemay

Universiteit Twente

Dr. Rob Hagmeijer

Universiteit Twente
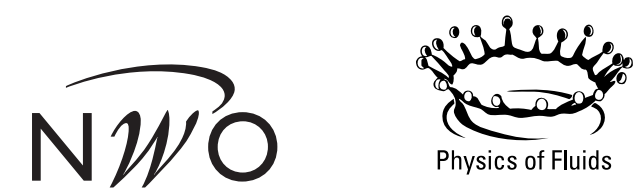

The work in this thesis was carried out at the Physics of Fluids group of the Faculty of Science and Technology of the University of Twente. It is funded by the Netherlands Organisation for Scientific Research (NWO) through VIDI Grant No. 11304.

Nederlandse titel:

Dynamica van vervormende druppels

Publisher:

Wilco Bouwhuis, Physics of Fluids, University of Twente, P.O. Box 217, 7500 AE Enschede, The Netherlands

pof.tnw.utwente.nl

Print: Gildeprint Drukkerijen, Enschede

(C) Wilco Bouwhuis, Enschede, The Netherlands, 2015

No part of this work may be reproduced by print

photocopy or any other means without the permission

in writing from the publisher.

ISBN: 978-90-365-3897-8

DOI: $10.3990 / 1.9789036538978$ 


\section{DYNAMICS OF DEFORMING DROPS}

\section{PROEFSCHRIFT}

ter verkrijging van

de graad van doctor aan de Universiteit Twente, op gezag van de rector magnificus,

Prof. dr. H. Brinksma,

volgens besluit van het College voor Promoties

in het openbaar te verdedigen

op vrijdag 28 augustus 2015 om 16.45 uur

door

Wilco Bouwhuis

geboren op 1 mei 1987

te Vroomshoop 
Dit proefschrift is goedgekeurd door de promotoren:

Prof. dr. Jacco H. Snoeijer

Prof. dr. Devaraj van der Meer 


\section{Contents}

1 Introduction 1

1.1 Deforming drops . . . . . . . . . . . . . . 1

1.2 Impact phenomena and the influence of the surrounding air . . 3

1.3 Leidenfrost drops . . . . . . . . . . . . . . . . . . 5

1.4 Drop deformation by laser-pulse impact . . . . . . . . . 7

1.5 The relevance of wetting properties . . . . . . . . . . 8

1.6 Guide through the thesis . . . . . . . . . . . . 10

2 Maximal air bubble entrainment at liquid drop impact $\quad 15$

2.1 Introduction . . . . . . . . . . . . . . 16

2.2 Interferometry experiments . . . . . . . . . . . . 16

2.3 Boundary Integral simulations . . . . . . . . . . . . . . . . . . 19

2.4 Scaling laws . . . . . . . . . . . . . . . . . . . . . . 23

2.5 Conclusion . . . . . . . . . . . . . . . . . . . . . 24

3 Universal mechanism for air entrainment during liquid im$\begin{array}{ll}\text { pact } & 27\end{array}$

3.1 Introduction . . . . . . . . . . . . . . . . . 28

3.2 Theory . . . . . . . . . . . . . . . . . . 30

3.2.1 Dimensional analysis and numerical method . . . . . . 30

3.2.2 Lubrication in moving and tilted coordinate system . . 33

Flow profile within the air film . . . . . . . . . 34

Solving the first order inhomogeneous ODE for $G(s) \ldots 36$

3.3 Results . . . . . . . . . . . . . . . . . . 37

3.3.1 Drop impact onto a pool . . . . . . . . . . . . 37

3.3 .2 Rigid sphere impact onto a pool . . . . . . . . . . 40

3.3.3 Deformations of interfaces: symmetrical behavior . . . . 41

3.4 Conclusion . . . . . . . . . . . . . . . 45

3.A Comparison $(r, z)$-system with $(n, s)$-system $\ldots \ldots \ldots$

3.B Continuity in curvilinear coordinates . . . . . . . . . 46 
3.C Sensitivity of the entrapped bubble volume to the rupture thick-

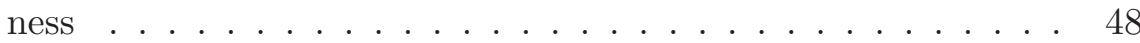

4 Initial surface deformations during impact on a liquid pool 53

4.1 Introduction . . . . . . . . . . . . . . . . . 54

4.2 Formulation . . . . . . . . . . . . . 56

4.2.1 Dimensional analysis . . . . . . . . . . . . . . 57

4.2.2 From gas pressure to interface deflection . . . . . . . 58

4.3 Results . . . . . . . . . . . . . . . . . . 60

4.3.1 Stokes gas flow . . . . . . . . . . . 60

4.3 .2 Potential gas flow . . . . . . . . . . . . . . . . 64

Large-gap regime: $h_{0} \gg R \ldots \ldots \ldots$. . . . . . 6 65

Thin-gap regime: $h_{0} \ll R \ldots \ldots 6$

Numerical simulations . . . . . . . . . . . . . 68

4.4 Conclusion . . . . . . . . . . . . . . . . . . 72

5 Impact of a high-speed train of microdrops on a liquid pool $\quad 77$

5.1 Introduction . . . . . . . . . . . . . . 78

5.1 .1 Background and motivation . . . . . . . . 78

5.1.2 Experiments: the creation and impact of a high velocity microdrop train . . . . . . . . . . . . . . . 79

5.1 .3 Objectives . . . . . . . . . . . . . . . 80

5.2 Problem statement and numerical method . . . . . . . 80

5.2.1 Parameters and assumptions . . . . . . . . . . 80

5.2 .2 Numerical method . . . . . . . . . . . . . . . . . 83

Boundary Integral simulations . . . . . . . . . . 83

The coalescence . . . . . . . . . . . . . 84

5.3 Results . . . . . . . . . . . . . . . . 85

5.3 .1 Comparison to experiments . . . . . . . . . 85

5.3 .2 Cavity shape . . . . . . . . . . . . . . . 88

5.3 .3 Cavity dynamics . . . . . . . . . . . . . . . . 91

5.4 Discussion . . . . . . . . . . . . . . . . . . . . . 94

5.A Solution of the cylindrical Rayleigh equation including the influence of surface tension . . . . . . . . . . . . . . . 96

6 Oscillating and star-shaped drops levitated by an airflow 103

6.1 Introduction . . . . . . . . . . . . . . . . . . . 104

6.2 Experimental setup . . . . . . . . . . . . . . . 106

6.3 Experimental results . . . . . . . . . . . . . . . . . . . 109 
6.3.1 Low-viscosity drops . . . . . . . . . . . . . . . . 109

6.3 .2 High-viscosity drops . . . . . . . . . . . . . . . . . 111

6.4 Numerical method . . . . . . . . . . . . . . . . . . 115

6.4.1 Parameters \& dimensional analysis . . . . . . . . . 115

6.4.2 Boundary Integral method coupled to lubricating gas layer 117

6.4.3 'Artificial' viscous damping . . . . . . . . . . . . . . 118

6.4 .4 Numerical details . . . . . . . . . . . . . . . . . . . 119

6.5 Numerical results . . . . . . . . . . . . . . . . . . . . 120

6.5.1 Steady shapes \& chimneys . . . . . . . . . . . 120

6.5 .2 Drop oscillations . . . . . . . . . . . . . . . 122

Observations . . . . . . . . . . . . . . 122

Stability diagram . . . . . . . . . . . . . . 126

Frequency analysis . . . . . . . . . . . . . 126

6.6 Discussion . . . . . . . . . . . . . . . . . . 127

7 Drop shaping by laser-pulse impact 133

7.1 Introduction . . . . . . . . . . . . . . . . . . 134

7.2 Experimental methods . . . . . . . . . . . . . . . 135

7.3 Results \& interpretation . . . . . . . . . . . . . . 137

7.4 Numerical results . . . . . . . . . . . . . . . . . . . . . . . 141

7.5 Conclusions . . . . . . . . . . . . . . . . . . 145

7.A Measurement of energy absorption . . . . . . . . . 145

7.B Measurement of drop displacement . . . . . . . . . . . 146

8 Effect of surface wettability on inertial pouring flows $\quad 151$

8.1 Introduction . . . . . . . . . . . . . . . . . . . 152

8.2 Model . . . . . . . . . . . . . . . . . . . . . 153

8.2.1 Definitions and assumptions . . . . . . . . . . 153

8.2 .2 Flow profiles and pressures . . . . . . . . . 156

8.2 .3 Calculating the forces . . . . . . . . . . . . . 159

8.2.4 Resulting system of equations . . . . . . . . . . 160

8.3 Results . . . . . . . . . . . . . . . . . . 161

8.3.1 Solutions . . . . . . . . . . . . . 161

8.3.2 Asymptotic expansion . . . . . . . . . . . . . . 164

8.4 Discussion . . . . . . . . . . . . . . . . 165

9 Summary and outlook $\quad 169$

9.1 Summary . . . . . . . . . . . . . . . . . . . . . 169

9.1.1 Small air bubble entrapment at liquid impact . . . . . 169 
9.1.2 High-speed microdrop train impact on a pool . . . . . . 171

9.1 .3 Levitated drops . . . . . . . . . . . . . . . . . . 171

9.1.4 Drop shaping by laser-pulse impact . . . . . . . . . . . 172

9.1.5 The effect of wettability on inertial pouring flows . . . . 172

9.2 Outlook . . . . . . . . . . . . . . . . 173

9.2.1 Small air bubble entrapment at liquid impact . . . . . . 173

9.2.2 High-speed microdrop train impact on a pool . . . . . . 174

9.2.3 Levitated drops . . . . . . . . . . . . . . . . . 175

9.2.4 Drop shaping by laser-pulse impact . . . . . . . . . . 175

$\begin{array}{ll}\text { Samenvatting (Nederlands) } & 177\end{array}$

$\begin{array}{ll}\text { List of scientific articles } & 181\end{array}$

$\begin{array}{lc}\text { Acknowledgements } & 183\end{array}$

$\begin{array}{ll}\text { About the author } & 191\end{array}$ 


\section{1 \\ Introduction}

\section{$1.1 \quad$ Deforming drops}

Liquid drops are everywhere around us. We encounter them in many situations in our daily lives - sometimes desirable, sometimes undesirable - and we make them play a dominant role in numerous industrial applications. Two obvious daily life examples are raindrops (Fig. 1.1a) and the drops falling from a dripping faucet (Fig. 1.1b). Examples of how we make use of drops are spray coating or spray painting (Fig. 1.1c), inkjet printing (Fig. 1.1d) [1], and spraying/sprinkling in agriculture or gardening (Fig. 1.1e) [2]. In all of the three latter examples, the generation, flight, impact, and spreading of the drops are separate stages of the corresponding industrial or agricultural processes, which researchers are trying to understand in full detail.

The shapes of falling drops through air has been thoroughly studied for many years [3-6]. Contrary to popular belief, drops that fall through the atmosphere do not have a pointy tear shape like a sessile drop sliding over a solid surface (inset of Fig. 1.1a). In the case of small raindrops with radius smaller than about $1 \mathrm{~mm}$, surface tension keeps the drop spherical. Larger drops deviate from this spherical shape, and evolve towards a 'pancake' shape, due to the flattening drag forces working on the drop during its fall. Very large falling drops are unstable and break into smaller drops, which is the 


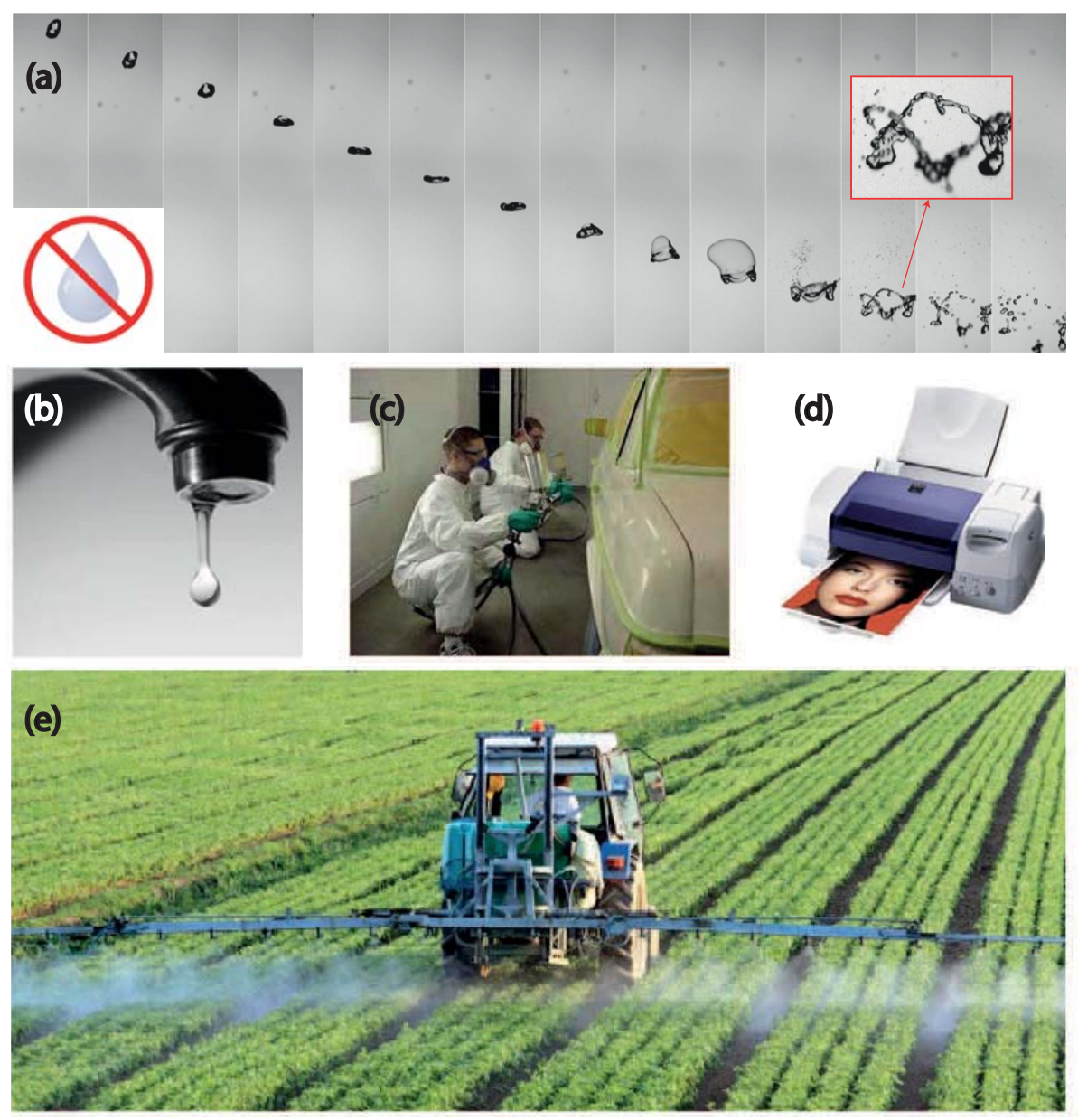

Figure 1.1: Five examples of drops in our daily life. (a) Raindrops; taken from Villermaux et al., 2009 [6]; inset: the 'popular' idea of the shape of a raindrop, which is incorrect; taken from http://www.wikipedia.com. (b) Drops dripping from a faucet; taken from http://www.popularmechanics.com/home/improvement/electricalplumbing/5-steps-to-fix-a-leaky-faucet-15470175. (c) Spray painting; taken from http://www.dudhopecoachworks.co.uk/Car-Spray-Painting.html. (d) Inkjet printing; taken from http://www.igraphicinc.com/how-do-inkjetprinters-work. (e) Spraying in agriculture - a common image in Twente; taken from http://natuurlijkgezondenmooi.blogspot.nl/2012/11/meest-enminst-bespoten-groente-en-fruit.html (web links as found on September 4 2014) 
scenario observed in the experiments of Fig. 1.1a. Raindrops are a perfect example of the fact that drops are highly susceptible to external forces, such as a surrounding airflow. The larger the drop, the more easy it is to deform the drop from its spherical equilibrium shape. This is what we will focus on in large part of this thesis: what is the influence of the external forces, in particular the flow of a surrounding gas, on the shape of the drop, within the context of impact onto a solid surface or a liquid pool? We will in particular look at the final stage of the impact: the stage just before the drop touches and starts to wet the surface on which it impacts. This stage turns out to be particularly interesting, because the influence of the flowing air between the drop and the surface strongly increases as soon as the air layer becomes narrow.

The title of this thesis, Dynamics of Deforming Drops, is now explained in a way in which the word 'deforming' is meant in a passive sense: drops can be deformed, and we will investigate the response of drops to external influences. We can also 'invert' the phenomenon of deformation and ask ourselves what happens with a liquid pool impacted by a liquid drop, or by a train of liquid drops (in the context of spray painting), just before and just after touch-down of the $\operatorname{drop}(\mathrm{s})$. In that case, the role of the word 'deforming' is meant in an active sense: the moving and impacting drops deform another liquid surface. This scenario will also be considered in this thesis.

\subsection{Impact phenomena and the influence of the sur- rounding air}

The most well-known impact phenomena are probably splashing and jet formation. However, the world of impact phenomena is much richer than these two effects, and Rein has given an extensive overview of a lot of possible impact scenarios [7]. Examples of other impact phenomena besides splashing and jetting are spreading and rim instability (for impact on a solid surface, i.e. wetting behavior) [8, 9], cavity formation and air bubble entrapment [10-13], and bouncing or partial coalescence $[14,15]$. These phenomena are influenced by several factors, such as the size of the impacting drop/object, its impact velocity, the liquid properties and inner flow, the solid properties (such as wettability and roughness), and the shape of the drop and the surface on the moment of impact.

Besides these obvious dependencies, one of the most striking discoveries in the field of drop impact is that the influence of the surrounding gas on 
(a)

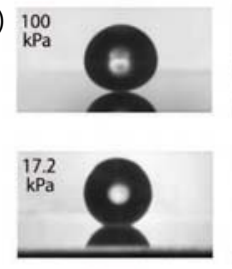

$0 \mathrm{~ms}$

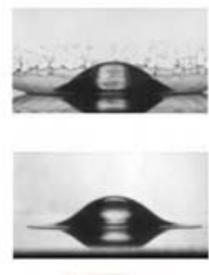

$0.552 \mathrm{~ms}$

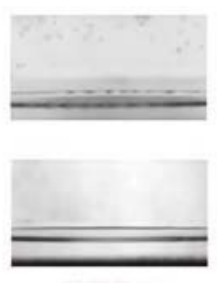

$2.484 \mathrm{~ms}$ (b)

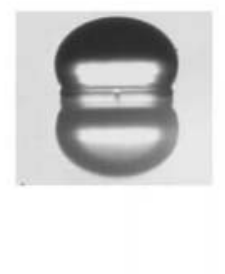

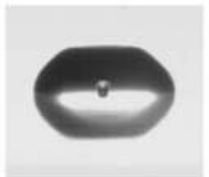

Figure 1.2: The crucial influence of the surrounding gas during impact of a liquid drop on a solid surface. (a) A decreased pressure in the surrounding gas completely suppresses a splash; taken from Xu et al., 2005 [16]. (b) Small air bubble entrapment caused by air film rupture; taken from Van Dam \& Le Clerc, 2004 [12]).

the impact phenomena is highly significant. $\mathrm{Xu}$ et al. found that, counter intuitively, decreasing the pressure of the surrounding gas can totally suppress splashing, as shown in Fig. 1.2a [16, 17]. The precise mechanism of this effect is still debated $[18,19]$. Another important effect of the surrounding gas is that it leads to a build-up of a localized pressure in the narrow layer of air (air film) in between the drop and the bottom surface, resulting in a very local deformation at the bottom of the drop [12, 20-22]. The rupture of the air film then leads to a small bubble entrapment at the front of the drop/object, as shown in Fig. 1.2b. Note that there are a number of other air bubble entrapment mechanisms for drop impact on a liquid pool, of which the most famous one is the so-called 'regular bubble entrainment'. The term 'regular' refers to the reproducibility of the effect $[10,11]$. Here, colliding small surface waves running over the cavity surface result in micrometer-sized bubbles, of which the pinch-off leads to the characteristic sound of raindrops impacting on a liquid surface. This well-known sound is thus not caused by the first impact of the drop on the water surface. Note that these 'regular' bubbles are typically left inside the liquid at the back of the impacting drop, i.e. at the bottom of the cavity.

Understanding the mechanisms of air bubble entrapment and the prediction of the sizes of the air bubbles left in the liquid are of great importance for many industries. In many applications, these bubbles are unwanted [12, 23]. In this thesis we will reveal the mechanism for bubble entrapment caused by air film rupture. How important is the surface tension of the liquid for this phenomenon? How do the drop size and impact speed influence the size of 
the entrapped bubble? How do the physics change when the impacting drop is replaced by an impacting (undeformable) solid sphere? In addition we will investigate the impact of a train of micrometer sized drops on a deep pool.

\subsection{Leidenfrost drops}

Next to impact, another example where drops are deformed by the surrounding gas is encountered for the Leidenfrost effect [24-26]. This arises when drops are levitated above a heated surface without touching it: at sufficiently large temperature, the drops are levitated by their own thin layer of vapor (Fig. 1.3b). This results in a highly increased lifetime of the drop (Fig. 1.3a) and these Leidenfrost drops are very mobile. The former is due to the decreased heat transfer from the plate to the liquid, since the vapor layer acts as a good insulator. The latter is due to the fact that there is no friction between the liquid and solid surface, which also implies that the drop is very susceptible to several kinds of instabilities. One of these instabilities is an air pocket breaking upwards through the liquid. This is called the 'chimney' instability, typically occurring at 'puddles' and drops larger than about $10 \mathrm{~mm}$ [25]. The threshold for chimneys is determined by an interplay between the viscosity of the gas layer, hydrostatics, and surface tension; the influence of the gas flux on the chimney threshold appears to be only small [27]. Remarkably in some sense, temperature is not explicitly included in the preceding list (it is implicitly, because the temperature gradients influence the evaporation rate, and thus the gas flow rate). Indeed, the chimney instability is a purely hydrodynamic instability, that can be reproduced by making the drops levitate above an airflow at room temperature [27, 28].

Another instability observed at Leidenfrost drops and levitated drops is the star drop instability (Fig. 1.3c) [28]. In particular large drops levitated by a large gas flux (or, in terms of Leidenfrost drops, with high evaporation rate) can spontaneously start to oscillate and break symmetry: they form oscillating star drops (Fig. 1.3c). The preferred mode number and frequency of the oscillation depends on the size of the drop, the gas flow velocity, and the liquid/gas properties. The fact that the star drop instability is also observed for levitated drops at room temperature again gives rise to the question how important the influence of temperature or heat transfer is for this phenomenon. Knowing the typical geometry of the drop and the gas layer below the drop from experiments, can we then also resolve the mechanism of the star drop instability by doing hydrodynamic simulations? The geometry of 
(a)
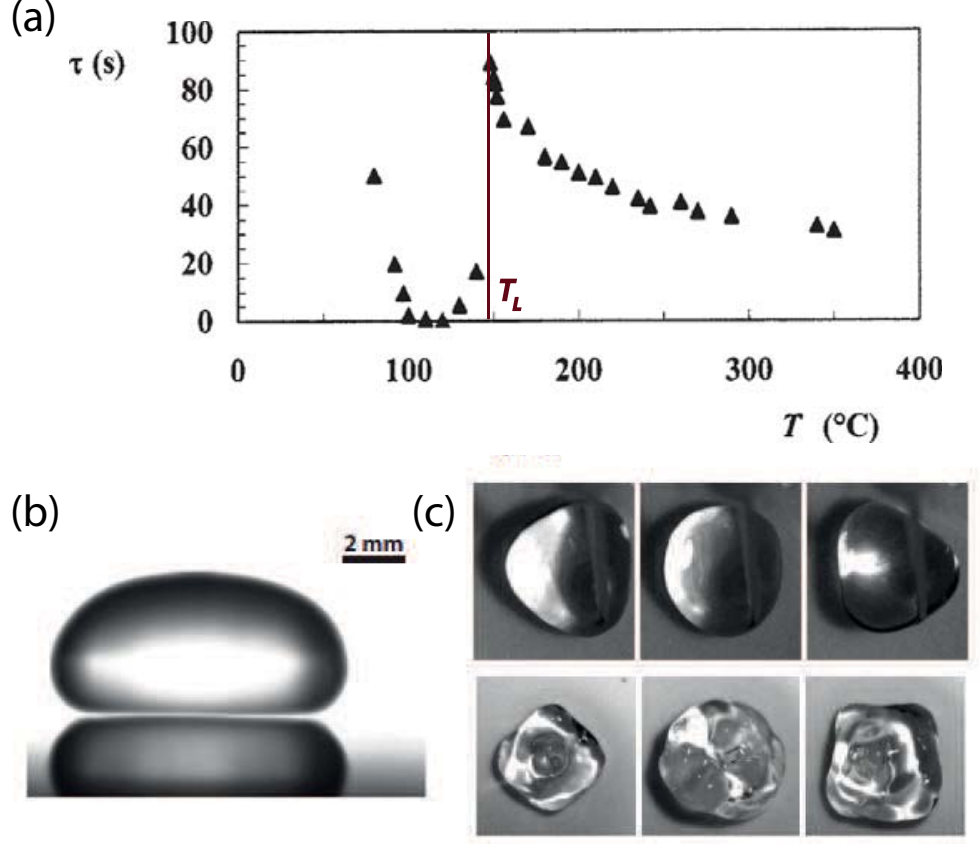

Figure 1.3: Levitated drops (Leidenfrost drops) and star drops. (a) The lifetime of an evaporating drop, the time it takes till the drop is completely evaporated, against the plate temperature $T$. At the 'Leidenfrost temperature' $T_{L}$, the lifetime of the drop suddenly increases significantly. Below the Leidenfrost temperature, drops remain 'sessile' (touching the surface), above the Leidenfrost temperature, drops are lifted from the surface by their own vapor layer. Taken from Biance et al., 2003 [25]. (b) A visualization of a 'medium sized' Leidenfrost drop. Taken from Quéré, 2013 [26]; courtesy of Raphaële Thévenin and Dan Soto. (c) Star drops levitated by an external airflow at room temperature. Taken from Brunet et al., 2011 [28]. 
the Leidenfrost problem is strongly reminiscent of the small air gap situations at drop impact in the preceding section. Again the gas exerts a local force on the drop - at its bottom, in particular - and an important question is whether this is sufficient to explain the instability towards Leidenfrost stars.

\subsection{Drop deformation by laser-pulse impact}

Apart from 'mechanical' impact, this thesis will also address the translation and deformation of a liquid drop impacted by a high-energy laser pulse. Besides the fact that this is very interesting from a fundamental point of view, and leads to beautiful visualizations (Fig. 1.4c), the research on this topic has its direct origin in industry. The link with industry is Extreme Ultraviolet (EUV) nanolithography, which we briefly introduce in this section.

Semiconductor manufacturing is all about reducing the size of the features that make up integrated circuit (IC) designs. Smaller features allow for faster and more advanced ICs that consume less power and can be produced at lower cost [29]. Over the years all electronic devices that we use, of which an obvious example is our mobile phone (Fig. 1.4a), became faster \& more sophisticated, and contained more and more data. To continue this trend, the world-leading company ASML in Veldhoven, The Netherlands, intensively works on the improvement of the IC resolution. In the latest technology, a laser-produced plasma source is used to generate EUV-light with a wavelength $13.5 \mathrm{~nm}$, which transfers a pattern from a mask to a light-sensitive chemical photo-resist on a semiconductor wafer $[29,30]$. The use of such a small wavelength further decreases the size of the smallest features on ICs. The plasma emitting the EUV results from falling liquid tin drops impacted by a nanosecond laserpulse, deforming the falling drop into a thin sheet, subsequently ionized by a second laser-pulse [29, 31] (Fig. 1.4b). A multilayer collector collects and focuses the light from the plasma onto the wafers. Maximizing the conversion of laser power to EUV power and minimizing the liquid tin debris requires a precise control of the drop shape, that is, understanding the fluid-dynamic response of a drop hit by a laser-pulse. This asks for a detailed understanding of the mechanism by which the laser moves or deforms the drop. To optimize the process of EUV generation, it is crucial to know how the translation, expansion, and fragmentation of the drop depend on the laser energy and the position of the drop with respect to the laser focus. 
(a)

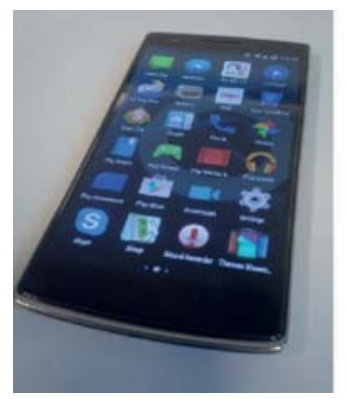

(b)

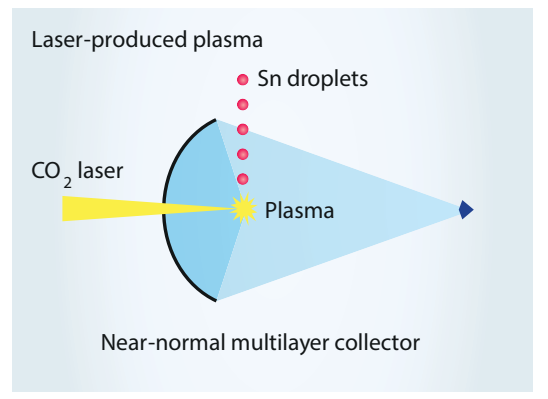

(c)

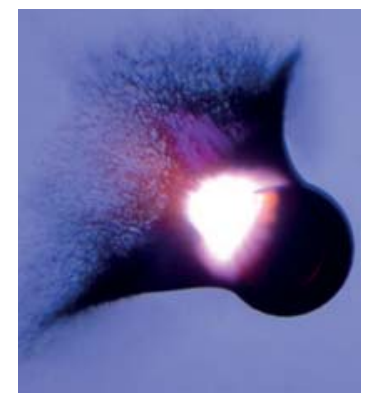

Figure 1.4: Generation of plasma by impact of a laser-pulse on a liquid drop and one of its applications. (a) A smart-phone, released April 2014. In the current society, phone electronics need to be faster and faster \& more and more sophisticated. Courtesy of Maarten Kok. (b) Sketch of an EUV source for lithography applications. Taken from Wagner and Harned, ASML, 2010 [29]. (c) Impact of a laser-pulse (wavelength $532 \mathrm{~nm}$ ) on a magenta-dyed water drop of radius $0.9 \mathrm{~mm}$, leading to a white plasma glow [32]. Courtesy of Alexander Klein.

\subsection{The relevance of wetting properties}

We have already introduced several impact scenarios. We also raised the question whether there is an equivalence between these scenarios. An equivalence between solid-liquid impact and liquid-liquid impact can not exist after the moment of touch-down between the liquid and the object: the wetting of a solid is a different process then a coalescence process. Thus, another relevant property determining the way a sphere impacts on a liquid or a drop impacts on a wall is the interaction between the solid and liquid surface. We distinguish between hydrophilic (water-attracting) surfaces and hydrophobic (water-repellent) surfaces, where hydrophobicity can be induced by chemical interactions or roughness on the scale from nanometer to micrometer. This roughness can have a natural origin [33, 34], but it can nowadays also be reproduced in industry - there are several examples of applications at which contact between a liquid and a solid needs to be avoided as much as possible (such as anti-corrosion, anti-icing, self-cleaning, and drag reduction). The wettability of a solid can be defined by the contact angle between the liquid and the solid, which quantifies how much a liquid drop at equilibrium tends to spread on a substrate $[35,36]$. 
It has been found that the impact of a hydrophilic sphere on a pool is completely different from the impact of a hydrophobic sphere on a pool. This is shown in Fig. 1.5a and b [37]. For otherwise identical experimental conditions, a hydrophilic impacting sphere smoothly sinks into the pool, while a hydrophobic sphere creates a huge splash. This difference is remarkable, since the behavior of the liquid on the millimeter scale of the object - and larger - is significantly influenced by the structures and interactions of the solid on scales that are smaller by orders of magnitude (nanometer to micrometer, as mentioned). The impact of a hydrophobic sphere on a pool has been investigated extensively $[37,38]$.
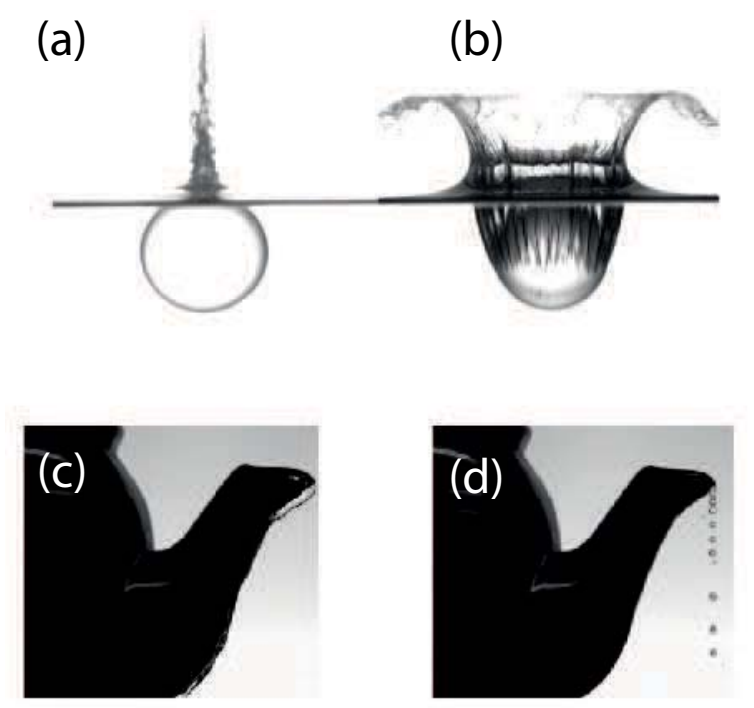

Figure 1.5: The relevance of wetting properties. (a), (b) Impact of a hydrophilic sphere (static contact angle about 15 degrees) and of a hydrophobic sphere (static contact angle about 100 degrees), respectively. The impact velocity is $5 \mathrm{~m} / \mathrm{s}$. The difference on the macro-scale is enormous; (a), (b), and corresponding data taken from Duez et al., 2007 [37]. (c) The 'teapot' effect: water trickling down the spout of a teapot. (d) Overcoming the teapot effect by using a hydrophobic surface; (c) and (d) taken from Duez et al., 2010 [41]. 
We will spend one chapter of this thesis on a similar phenomenon where the influence of small scale structures plays a crucial role, the so-called 'teapot effect'. We all know the - quite annoying - tendency of a poured liquid to follow a curved solid surface, and trickle down the spout of a teapot or bottle, to land on a different place than where it should (Fig. 1.5c). The reason for this trickling is the so-called Coanda effect: for fast streaming, the flow velocity just above the solid is larger than at the top of the liquid flow, which sets a Bernoulli pressure difference over the film, pushing back the liquid along the spout [39-41]. Duez et al. found that by using a hydrophobic surface at the spout, this effect could be completely suppressed [41] (Fig. 1.5d). A qualitative explanation and scaling law, including the huge separation of length scales, was included - the contact angle acts like a very local boundary condition, indeed influencing the large scale flow - but a more detailed theory was missing. For example, there exists a critical flow speed, below which all liquid trickles down the spout, and no stable jet can exist [41], but the transition was not predicted. We will investigate the dependence of the trickling transition on the flow speed, film thickness, and contact angle.

\subsection{Guide through the thesis}

In Chapters 2-5, we will focus on impact. In Chapter 2, we address the mechanism of small air bubble entrapment for impact of a liquid drop on a solid surface, and we show how to predict the size of the small air bubble left in the liquid after impact. In Chapter 3, we make the step to the other two impact scenarios: impact of a liquid drop onto a pool, and impact of a solid sphere onto a pool, and point out the equivalences/symmetries between the air bubble entrapment in these different situations. In Chapter 4, we consider the very first deformations of a pool surface, approached by a solid sphere. These deformations can be predicted analytically, and we identify different regimes where either viscosity or inertia of the gas plays a crucial role. In Chapter 5, we leave the small bubble entrainment and study the impact of a train of droplets on a pool, focusing on the shape and dynamics of the cavity that emerges during the impact.

In Chapter 6, we show how we reproduce the star-drop instability of levitated drops and Leidenfrost drops using hydrodynamic simulations, without any influence of temperature and heat transfer. Chapter 7 will focus on the dynamical response of drops exposed by a laser, or, more generally, the dynamics of drops due to a localized forcing. In Chapter 8, we step out of the 
world of drops, to investigate inertial pouring flows and the trickling transition observed in, for example, teapot flow.

Finally, Chapter 9 contains our overall conclusions and gives an overview of possible future studies.

\section{References}

[1] O. A. Basaran, H. Gao, and P. P. Bhat, "Nonstandard inkjets", Annu. Rev. Fluid Mech. 45, 85-113 (2013).

[2] S. Ghosh and J. C. R. Hunt, "Spray jets in a cross flow", J. Fluid Mech. 365, 109-136 (1998).

[3] A. F. Spilhaus, "Raindrop size, shape, and falling speed", J. Meteor. 5, 108-110 (1948).

[4] D. M. A. Jones, "The shape of raindrops", J. Meteor. 16, 504-510 (1959).

[5] K. V. Bird and C. Chuang, "A new model for the equilibrium shape of raindrops", J. Atmos. Sci. 44, 1509-1524 (1987).

[6] E. Villermaux and B. Bossa, "Single-drop fragmentation determines size distribution of raindrops", Nature Phys. 5, 697-702 (2009).

[7] M. Rein, "Phenomena of liquid drop impact on solid and liquid surfaces", Fluid Dyn. Res. 12, 61-93 (1993).

[8] D. C. Vadillo, A. Soucemarianadin, C. Delattre, and D. C. D. Roux, "Dynamic contact angle effects on the maximum drop impact spreading on solid surfaces", Phys. Fluids 21, 122002 (2009).

[9] J. Eggers, M. A. Fontelos, C. Josserand, and S. Zaleski, "Drop dynamics after impact on a solid wall: Theory and simulations", Phys. Fluids 22, 062101 (2010).

[10] H. N. Oguz and A. Prosperetti, "Bubble entrainment by the impact of the impact of drops on liquid surfaces", J. Fluid Mech. 219, 143-179 (1990).

[11] H. C. Pumphrey and P. A. Elmore, "The entrainment of bubbles by drop impacts", J. Fluid Mech. 220, 539-567 (1990). 
[12] D. B. van Dam and C. Le Clerc, "Experimental study of the impact of an ink-jet printed droplet on a solid substrate", Phys. Fluids 16, 3403-3414 (2004).

[13] T. Tran, H. de Maleprade, C. Sun, and D. Lohse, "Air entrainment during impact of droplets on liquid surfaces", J. Fluid Mech. 726, R3 (2013)

[14] A. Eddi, A. Boudaoud, and Y. Couder, "Oscillating instability of bouncing droplet crystals", EPL 94, 20004 (2011).

[15] J. Molácek and J. W. M. Bush, "Drops bouncing on a vibrating bath", J. Fluid Mech. 727, 582-611 (2013).

[16] L. Xu, W. W. Zhang, and S. R. Nagel, "Drop splashing on a dry smooth surface", Phys. Rev. Lett. 94, 184505 (2005).

[17] L. Xu, L. Barcos, and S. R. Nagel, "Splashing of liquids: Interplay of surface roughness with surrounding gas", Phys. Rev. E. 76, 066311 (2007).

[18] M. M. Driscoll and S. R. Nagel, "Ultrafast interference imaging of air in splashing dynamics", Phys. Rev. Lett. 107, 154502 (2011).

[19] S. Mandre and M. P. Brenner, "The mechanism of a splash on a dry solid surface", J. Fluid Mech. 690, 148-172 (2012).

[20] S. T. Thoroddsen, T. G. Etoh, K. Takehara, N. Ootsuka, and A. Hatsuki, "The air bubble entrapped under a drop impacting on a solid surface", J. Fluid Mech. 545, 203-212 (2005).

[21] P. D. Hicks and R. Purvis, "Air cushioning and bubble entrapment in three-dimensional droplet impacts", J. Fluid Mech. 649, 135-163 (2010).

[22] M. Mani, S. Mandre, and M. P. Brenner, "Events before droplet splashing on a solid surface", J. Fluid Mech. 647, 163âĂŞ185 (2010).

[23] D. L. Keij, K. G. Winkels, H. Castelijns, M. Riepen, and J. H. Snoeijer, "Bubble formation during the collision of a sessile drop with a meniscus", Phys. Fluids 25, 082005 (2013).

[24] J. G. Leidenfrost, De Aquae Communis Nonnullis Qualitatibus Tractatus, Duisburg on Rhine, Germany (1756).

[25] A.-L. Biance, C. Clanet, and D. Quéré, "Leidenfrost drops", Phys. Fluids 15, 1632-1637 (2003). 
[26] D. Quéré, "Leidenfrost dynamics", Annu. Rev. Fluid Mech. 45, 197-215 (2013).

[27] J. H. Snoeijer, P. Brunet, and J. Eggers, "Maximum size of drops levitated by an air cushion", Phys. Rev. E 79, 036307 (2009).

[28] P. Brunet and J. H. Snoeijer, "Star drops formed by periodic excitation and on an air cushion - A short review", Eur. Phys. J. Spec. Top. 192, 207-226 (2011).

[29] C. Wagner and N. Harned, "Lithography gets extreme", Nature Phot. 4, 24-26 (2010).

[30] V. Y. Banine, G. H. P. M. Swinkels, and K. N. Koshelev, "Physical processes in EUV sources for microlithography", J. Phys. D: Appl. Phys. 44, 253001 (2011).

[31] H. Mizoguchi, T. Abe, Y. Watanabe, T. Ishihara, T. Ohta, T. Hori, T. Yanagida, H. Nagano, T. Yabu, S. Nagai, G. Soumagne, A. Kurosu, K. M. Nowak, T. Suganuma, M. Moriya, K. Kakizaki, A. Sumitani, H. Kameda, H. Nakarai, and J. Fujimoto, "100W 1st generation laser-produced plasma light source system for HVM EUV lithography", Proc. SPIE 7636, 76308 (2010).

[32] A. L. Klein, W. Bouwhuis, C. W. Visser, H. Lhuissier, C. Sun, J. H. Snoeijer, E. Villermaux, D. Lohse, and H. Gelderblom, "Drop shaping by laser-pulse impact", Phys. Rev. Appl. 3, 044018 (2015),

See Chapter 7 of this thesis.

[33] K. Koch, B. Bhushan, and W. Barthlott, "Diversity of structure, morphology and wetting of plant surfaces", Soft Matter 4, 1943-1963 (2008).

[34] N. Hornsveld, "Characterisation of natural epicuticular wax", Master Thesis Applied Physics, Physics of Interfaces and Nanomaterials (PIN) group, Faculty of Science \& Technology, University of Twente (2014).

[35] P.-G. de Gennes, F. Brochard-Wyart, and D. Quéré, Capillarity and wetting phenomena; Drops, bubbles, pearls, waves, Springer (2004).

[36] J. H. Snoeijer and B. Andreotti, "Moving contact lines: Scales, regimes, and dynamical transitions", Annu. Rev. Fluid Mech. 45, 269-292 (2013). 
[37] C. Duez, C. Ybert, C. Clanet, and L. Bocquet, "Making a splash with water repellency", Nature Phys. 3, 180-183 (2007).

[38] J. M. Aristoff and J. W. M. Bush, "Water entry of small hydrophobic spheres", J. Fluid Mech. 619, 45-78 (2009).

[39] J.-M. Vanden-Broeck and J. B. Keller, "Pouring flows", Phys. Fluids 29, 3958-3961 (1986).

[40] J.-M. Vanden-Broeck and J. B. Keller, "Pouring flows with separation", Phys. Fluids A 1, 156-158 (1989).

[41] C. Duez, C. Ybert, C. Clanet, and L. Bocquet, "Wetting controls separation of inertial flows from solid surfaces", Phys. Rev. Lett. 104, 084503 (2010). 


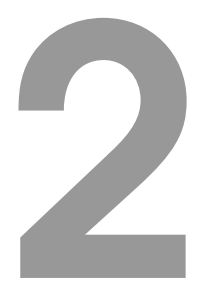

\section{Maximal air bubble entrainment at liquid drop impact $* \dagger$}

At impact of a liquid drop on a solid surface an air bubble can be entrapped. Here we show that two competing effects minimize the (relative) size of this entrained air bubble: for large drop impact velocity and large droplets the inertia of the liquid flattens the entrained bubble, whereas for small impact velocity and small droplets capillary forces minimize the entrained bubble. However, we demonstrate experimentally, theoretically, and numerically that in between there is an optimum, leading to maximal air bubble entrapment. For a 1.8 $\mathrm{mm}$ diameter ethanol droplet this optimum is achieved at an impact velocity of $0.25 \mathrm{~m} / \mathrm{s}$. Our results have a strong bearing on various applications in printing technology, microelectronics, immersion lithography, diagnostics, or agriculture.

${ }^{*}$ Published as: W. Bouwhuis, R.C.A. van der Veen, T. Tran, D.L. Keij, K.G. Winkels, I.R. Peters, D. van der Meer, C. Sun, J.H. Snoeijer, D. Lohse, "Maximal air bubble entrainment at liquid-drop impact", Phys. Rev. Lett. 109, 264501 (2012).

${ }^{\dagger}$ The numerical simulations in this chapter are part of the present thesis. The experimental work is due to Roeland van der Veen. 


\section{$2.1 \quad$ Introduction}

The impact of liquid droplets on surfaces is omnipresent in nature and technology, ranging from falling raindrops to applications in agriculture and inkjet printing. The crucial question often is: how well does the liquid wet a surface? The traditional view is that it is the surface tension which gives a quantitative answer. However, it has been shown recently that an air bubble can be entrapped under a liquid drop as it impacts on the surface [1-6]. Also $\mathrm{Xu}$ et al. $[7,8]$ revealed the important role of the surrounding air on the impact dynamics, including a possible splash formation. The mechanism works as follows [3-6]: the air between the falling drop and the surface is strongly squeezed, leading to a pressure buildup in the air under the drop. The enhanced pressure results in a dimple formation in the droplet and eventually to the entrapment of an air bubble (Fig. 2.1a). The very simple question we ask and answer in this chapter is: for which impact velocity is the entrapped bubble maximal?

\section{$2.2 \quad$ Interferometry experiments}

Our experimental setup is shown in Fig. 2.1b and is similar to that of Refs. [9, 10] where it is described in detail. An ethanol drop impacts on a smooth glass surface after detaching from a needle, or for velocities smaller than $0.32 \mathrm{~m} / \mathrm{s}$, after moving the needle downwards using a linear translation stage. A highspeed side view recording is used to measure the drop diameter and velocity. The experiment is carried out at room temperature. A synchronized bottom view recording by a high-speed color camera is used to measure the deformed shape of the liquid drop. Colored interference patterns are created by highintensity coaxial white light, which reflects from both the glass surface and the bottom of the droplet. Using a color-matching approach in combination with known reference surfaces, the complete air thickness profile can be extracted (shown in Fig. 2.1c). For experiments done at larger impact velocities $(U>0.76 \mathrm{~m} / \mathrm{s})$, we use a pulse of diffused laser light triggered by an optical switch. The thickness of the air film at the rim is assumed to be zero, and the complete air thickness profile can then be obtained from the monochromatic fringe pattern. From these measurements we can determine the dimple height, $H_{d}$, and the volume of the entrained bubble, $V_{b}$, at the very moment of impact. This moment is defined by the first wetting of the surface. This is the moment when the concentric symmetry of the interference rings is lost, 
since due to unavoidable tiny tilts of the glass plate the wetting in general is non-axisymmetric. To calculate the bubble volume $V_{b}$, we integrate the thickness profile of the air layer trapped beneath the drop. Note that the dimple profiles and the volume of the entrained bubble are obtained before the wetting occurs, such that we do not have to take into account the properties of the surface (e.g., contact angle or roughness, which is of the order of $10 \mathrm{~nm}$ ). Alternatively, we can also measure the volume of the trapped bubble after impact when the liquid already wets the surface. Both measurements provide the same results. In the present chapter, we use the first approach.

(a)

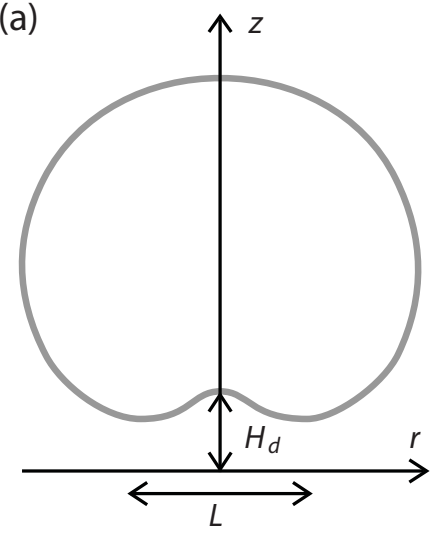

(b)

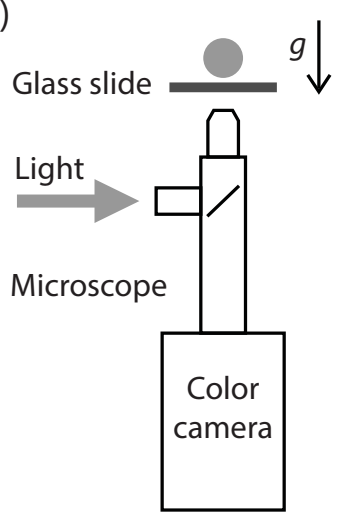

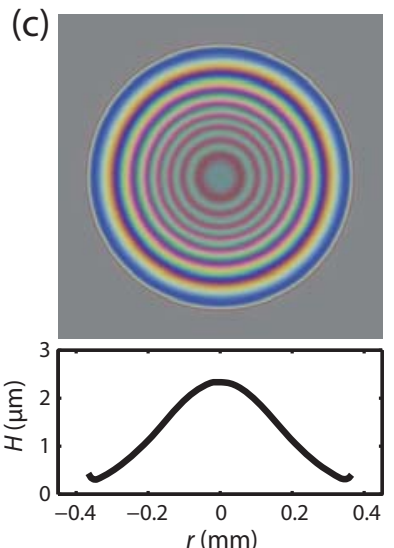

Figure 2.1: Experimental characterization of air bubble entrapment. (a) Sketch of dimple formation (not drawn to scale) just prior to impact. (b) Schematic of the experimental setup used to study droplet impact on smooth surfaces. An ethanol droplet of typical radius $R=0.9 \mathrm{~mm}$ falls on a glass slide of average roughness $10 \mathrm{~nm}$. The impact velocity is varied by varying the falling height of the droplet. For very small velocities below $0.31 \mathrm{~m} / \mathrm{s}$, the droplet is fixed at the tip of $0.4 \mathrm{~mm}$-diameter capillary that is vertically translated downwards at a constant velocity. The bottom view is captured by a high-speed color camera (SA2, Photron Inc.). The camera is connected to a long working-distance microscope and a $5 \times$ objective to obtain a $2 \mathrm{~mm}$ field of view. (c) An example of an interference pattern and the extracted air thickness profile. Note the difference in horizontal and vertical length scales. The exposure time was $1 / 15000 \mathrm{~s}$ and the typical frame rate of the recordings is 5000 frames per second. 
The results are shown in Fig. 2.2. Clearly, both dimple height at impact and the size of the entrained bubble have a pronounced maximum as function of the impact velocity $U$. The corresponding impact velocity for which the air entrainment is maximal is in the regime $U_{0}=0.1-0.25 \mathrm{~m} / \mathrm{s}$ for an ethanol droplet of radius $R=0.9 \mathrm{~mm}$ (or the Stokes number $\mathrm{St}_{0}=0.3 \times 10^{4}-1 \times 10^{4}$ ). While length scales are given in multiples of the droplet radius $R$, following Brenner et al. $[3,6]$ we express the impact velocity $U$ in terms of the Stokes number St, defined with the dynamic air viscosity $\eta_{g}=1.82 \times 10^{-5} \mathrm{~Pa} \cdot \mathrm{s}$ and the liquid density $\rho_{l}=789 \mathrm{~kg} / \mathrm{m}^{3}$ as St $=\rho_{l} R U / \eta_{g}=\rho_{l} / \rho_{g}$ Re, where Re $=$ $\rho_{g} R U / \eta_{g}$ is the standard Reynolds number of the gas. A further relevant parameter of the system is the surface tension $\gamma=22 \mathrm{mN} / \mathrm{m}$, which can be expressed in terms of the Weber number $\mathrm{We}=\rho_{l} R U^{2} / \gamma$ or in terms of the capillary number $\mathrm{Ca}=\eta_{g} U / \gamma=\mathrm{We} / \mathrm{St}$.
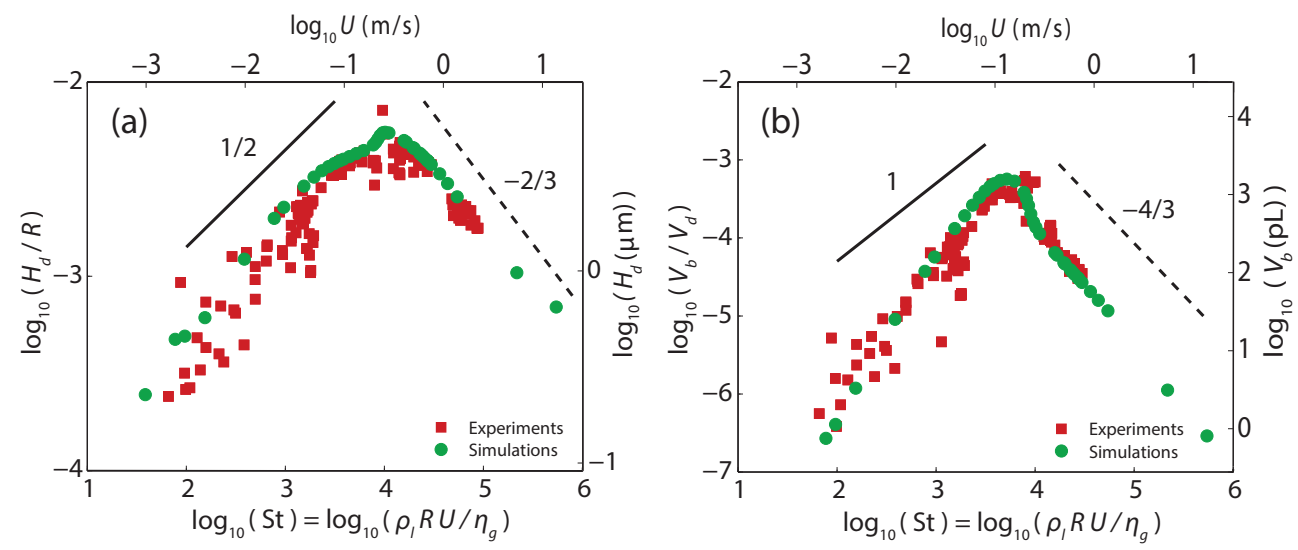

Figure 2.2: Maximum entrapment of air bubbles. (a) Dimple height $H_{d}$ and (b) entrained bubble volume $V_{b}$ as functions of the impact velocity $U$ (upper axes) and the Stokes number St (lower axes). The shape of the air layer can be characterized by the dimple height $H_{d}$ and the lateral extension $L$. Red squares correspond to high-speed color interferometry measurements, green dots correspond to numerical simulations. The straight lines correspond to the derived scaling laws in the capillary regime (solid) and inertial regime (dashed) with the respective scaling exponents. 


\subsection{Boundary Integral simulations}

We compare and supplement our experimental findings on the dimple height at impact and the entrained bubble size to numerical results. The numerical simulation consists of an axisymmetric boundary integral (BI) simulation for the liquid droplet in which the droplet is assumed to obey potential flow, coupled to a lubrication approximation of the Stokes equation

$$
\frac{\partial P_{g}}{\partial r} \sim \eta_{g} \frac{\partial^{2} u_{r}}{\partial z^{2}}
$$

that describes the viscous, incompressible gas flow under the droplet $[3,11-$ 14]. Here, $z$ is the vertical direction, $P_{g}(r, t)$ is the gas pressure, while $u_{r}$ is the radially outward velocity in the gas parallel to the surface (Fig. 2.1a). Note that the gas flow under the droplet is indeed viscous: an upper bound for the Reynolds number relevant for the lubrication flow gives $U H_{d} / \nu_{g} \sim 0.1$ for the highest impact velocity, and is typically much smaller for most of our experiments.

We now give more details on the numerical simulation: the velocity field inside the droplet is described with a scalar velocity potential $\phi$, obeying the Laplace equation $\nabla^{2} \phi=0$. The axisymmetric droplet contour is described using cylindrical coordinates $r, z$ and is solved numerically by using the BI method; the simulations are based on the numerical code described by Refs. [15-17]. This BI simulation is an alternative way of solving the system of equations, compared to the method applied by Mani et al., 2010 [11], in which case a Hilbert transform method was applied. In contrast to Eggers et al., 2010 [14], we do not solve the complete Navier-Stokes equations, but do include dynamics of the air layer below the drop. The dynamic boundary condition valid on the droplet contours is given by the unsteady Bernoulli equation,

$$
\left(\frac{\partial \phi}{\partial t}+\frac{1}{2}|\nabla \phi|^{2}\right)=-g z-\frac{\gamma}{\rho_{l}} \kappa(r, t)-\frac{P_{g}(r, t)-P_{\infty}}{\rho_{l}} .
$$

Here $t$ is time, $g$ the acceleration of gravity, $z$ the absolute height, $\kappa(r, t)$ the interface curvature, and $P_{\infty}$ the far-field pressure. The key dynamical quantities in $(2.2)$ are the gas pressure $P_{g}(r, t)$ and the interface curvature $\kappa(r, t)$. The curvature is related to the dimple profile $H(r, t)$ by the geometric 
relation

$$
\kappa(r, t)=\frac{\frac{\partial^{2} H(r, t)}{\partial r^{2}}}{\left(1+\left(\frac{\partial H(r, t)}{\partial r}\right)^{2}\right)^{3 / 2}}+\frac{\frac{\partial H(r, t)}{\partial r}}{r\left(1+\left(\frac{\partial H(r, t)}{\partial r}\right)^{2}\right)^{1 / 2}} .
$$

To close the problem, an additional equation is provided by the lubrication approximation for the viscous gas flow at the bottom of the droplet,

$$
\frac{\partial H(r, t)}{\partial t}-\frac{1}{r} \frac{\partial}{\partial r}\left[\frac{r(H(r, t))^{3}}{12 \eta_{g}} \frac{\partial P_{g}(r, t)}{\partial r}\right]=0,
$$

with boundary condition $\left.P_{g}\right|_{r=R}=P_{\infty}$; the gas pressure at the top of the droplet is set to atmospheric. Contrarily to Mani et al., 2010 [11], we do not incorporate effects of compressibility of the gas, since, following the analysis of Hicks et al., 2011 [13], there is little influence of compressibility in the regime that is studied here. The initial conditions for the simulations consist of a spherical droplet with radius $R$ having a downward velocity $U$. The initial height is taken sufficiently high for the pressure induced by the radial velocity profile to be still negligible as compared to the ambient pressure $(\sim 10 \mu \mathrm{m})$. The number of nodes on the droplet surface for which the BI equations are solved is of order 100, with node density increasing for $r \rightarrow 0$. The number of nodes and the size of the time steps vary during the simulation as a function of the local gap height and velocity of the droplet contour. The size of a time step is of order $10 \mathrm{~ns}$. For any number of nodes, the coupling between gap height and pressure profile breaks down for some small value of $H$, since the pressure diverges at vanishing thickness of the air layer. Consistent with the experimental resolution we continue our simulations until the minimum gap thickness reaches $0.4 \mu \mathrm{m}$, while ensuring that our algorithm remains accurate. This is the moment at which the values for $H_{d}$ and $V_{b}$ are extracted, which, as we will show below, have already achieved their final value much earlier.

Figure 2.3 shows the evolutions of the simulated ethanol droplets (blue lines). The two panels correspond to $U=0.361 \mathrm{~m} / \mathrm{s}$ and $U=0.763 \mathrm{~m} / \mathrm{s}$ (both at the right side of the maximum in Fig. 2.2) and are compared directly with the one-frame-results from experiment (red line). The comparison involves no adjustable parameters and reveals an excellent agreement for the dimple height. Given these satisfactory results, we can use the simulations to obtain further information of the time evolution of the air layer. Figure 2.4 shows the dimple height $H_{d}$ (solid line) and the minimum gap height $d$ (dashed line) as a function of time. Here, $t=0$ is defined by the moment at which the drop 

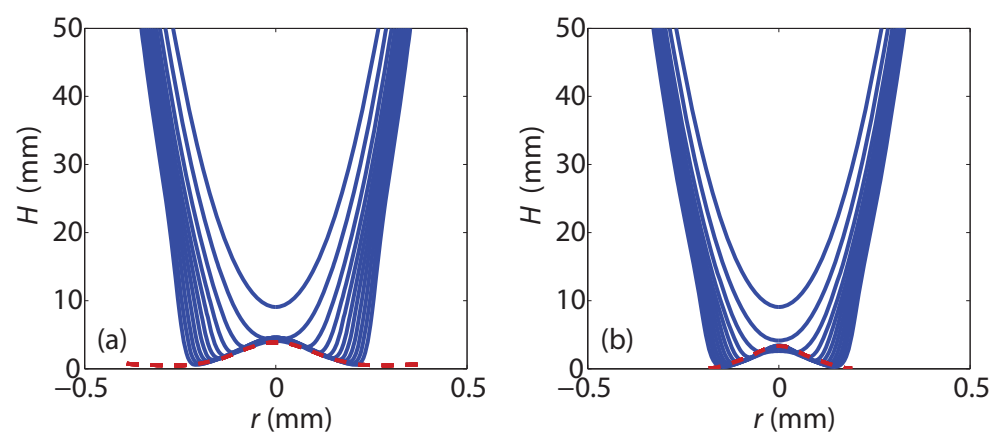

Figure 2.3: BI time evolution (solid blue lines) and an experimental profile (dashed red line) for ethanol droplet impact at (a): $U=0.32 \mathrm{~m} / \mathrm{s}(\mathrm{St}=$ $\left.1.25 \times 10^{4}\right)$ and $(\mathrm{b}): U=0.76 \mathrm{~m} / \mathrm{s}\left(\mathrm{St}=2.97 \times 10^{4}\right)$.
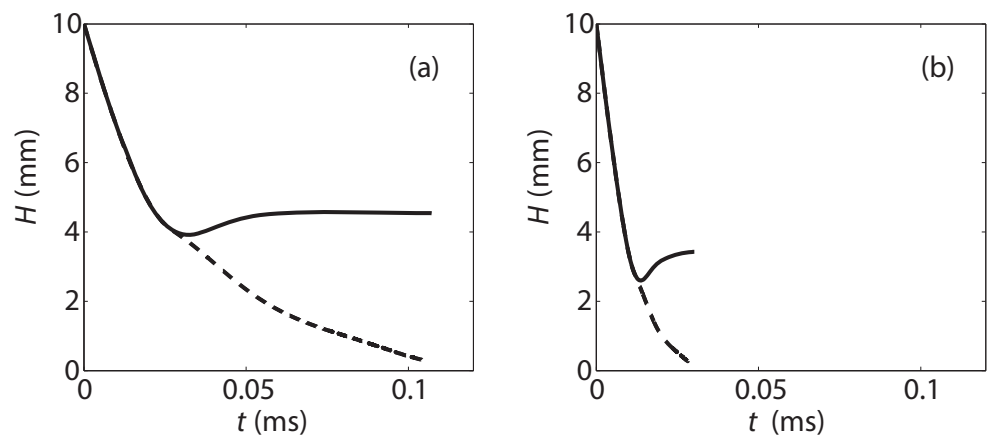

Figure 2.4: Time evolution of the height $H_{d}$ of the dimple (solid line) and the distance $d$ between the closest point of the liquid surface and the solid surface (dashed line) for the impact velocities (a): $U=0.32 \mathrm{~m} / \mathrm{s}$ and (b): $U=0.76 \mathrm{~m} / \mathrm{s}$. For both cases it is visible that the final dimple height is achieved way before the simulation is stopped at the cut-off $d=0.4 \mu \mathrm{m}$. 
passes $H_{d}=10 \mu \mathrm{m}$. The separation of the solid and dashed lines marks the moment at which the center height, $H(r=0)$, no longer represents a minimum but has turned into a local maximum. This feature can also be inferred from the drop profiles shown in Fig. 2.3. The dimple height remains approximately constant at the later stages of Fig. 2.3. This implies that it is not critical to know the exact time at which the experimental profile is determined: the value of $H_{d}$ is not expected to vary much in this stage of the experiment.
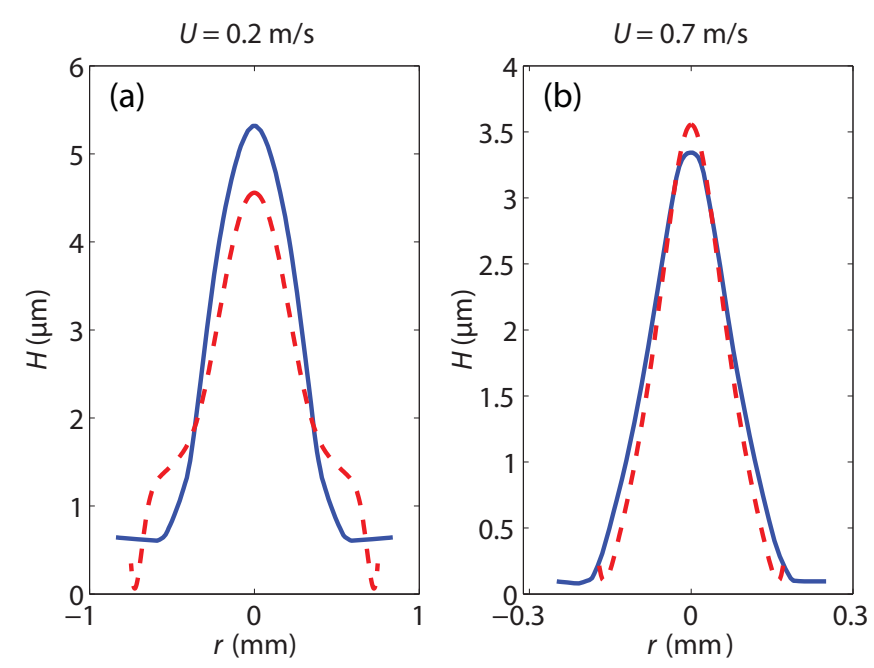

Figure 2.5: Comparison of experimental (blue) and numerical (red) dimple profiles for two different impact velocities; (a): $U=0.2 \mathrm{~m} / \mathrm{s}\left(\mathrm{St}=7.8 \times 10^{3}\right.$; crossover regime) and (b): $U=0.7 \mathrm{~m} / \mathrm{s}\left(\mathrm{St}=2.7 \times 10^{4}\right.$; inertial regime).

The results of the numerical calculations of the dimple height and bubble volume are shown in Fig. 2.2, together with the experimental data, showing very good agreement: in particular, we observe pronounced maxima in the dimple size and in the entrained bubble volume. In the numerically obtained dimple height (and volume, to a lesser extent), we observe a jump exactly at the crossover regime. This jump originates from a change in the shape of the dimple. We focus on this in Fig. 2.5, which compares the experimental and numerical dimple profiles for an impact velocity at the crossover regime $(U=0.2 \mathrm{~m} / \mathrm{s})$ and an impact velocity in the inertial regime $(U=0.7 \mathrm{~m} / \mathrm{s})($ these are parameters different from the ones chosen in Fig. 2.3 and Fig. 2.4). While the profiles are in excellent agreement within the St regime (both volume and dimple height), the numerical profile develops a "double dimple" at the 
crossover impact speed. Within the low St regime, we do not observe this double dimple, but the dimple is typically much broader. The variation in dimple shape results in the jump observed for the numerical dimple heights in the crossover regime (see Fig. 2.2a). In all cases, however, the dimple height $H_{d}$ and the entrapped bubble volume $V_{b}$ are in quantitative agreement without any adjustable parameters.

\subsection{Scaling laws}

Numerical and experimental results together suggest scaling laws $H_{d} / R \sim$ $\mathrm{St}^{-2 / 3}$ for larger Stokes numbers, while $H_{d} / R \sim \mathrm{St}^{1 / 2}$ for smaller Stokes numbers. We will now theoretically derive these scaling laws. For large St we follow and extend Refs. [6, 12, 18]: the horizontal length scale $L$ of the dimple extension (see Fig. 2.1a) follows from geometrical arguments as $L \sim \sqrt{H_{d} R}$, and $u_{r}$ from mass conservation as $u_{r} \sim U L / H_{d}$. The Stokes equation (2.1) suggests $P_{g} \sim L \eta_{g} u_{r} / H_{d}^{2}$ as estimate for the gas pressure below the falling drop at touch-down. The liquid pressure $P_{l}$ can be estimated from the unsteady Bernoulli equation: dimensional analysis gives the deceleration timescale $H_{d} / U$ and the potential in the liquid $\sim U L$, resulting in $P_{l} \sim \rho_{l} U^{2} L / H_{d}$. Since the liquid drop will be deformed when $P_{g} \sim P_{l}$, one finally obtains the scaling for the dimple height and the bubble volume:

$$
H_{d} \sim R \mathrm{St}^{-2 / 3}, \quad V_{b} \sim L^{2} H_{d} \sim R^{3} \mathrm{St}^{-4 / 3} .
$$

This describes the air bubble in the inertial regime, i.e. large impact velocities, in agreement with our experimental and numerical findings.

For small St, corresponding to small impact velocity and small droplet radius, capillarity will take over and try to smoothen the dimple out. Then the pressure inside the gas must be balanced with the Laplace pressure $\gamma \kappa$ that is imposed at the liquid-air interface, where $\kappa \sim H_{d} / L^{2}$ is the curvature of the dimple. Using once more that the gas pressure $P_{g} \sim L \eta_{g} u_{r} / H_{d}^{2}$, one obtains

$$
\begin{aligned}
\frac{H_{d}}{R} & \sim \sqrt{\mathrm{Ca}} \sim \sqrt{\mathrm{We} / \mathrm{St}} \sim \frac{\eta_{g}}{\sqrt{\gamma \rho_{l} R}} \mathrm{St}^{1 / 2}, \\
\frac{V_{b}}{R^{3}} & \sim \frac{\eta_{g}^{2}}{\gamma \rho_{l} R} \mathrm{St},
\end{aligned}
$$

as scaling in the capillary regime. Again, this is consistent with the experimental and numerical findings. The crossover between the regimes, corresponding 
to the maximal air bubble entrainment, occurs at

$$
\mathrm{St}_{o} \sim \mathrm{Ca}_{o}^{-3 / 4} \quad \text { or } \quad U_{o} \sim \frac{\eta_{g}^{1 / 7} \gamma^{3 / 7}}{\rho_{l}^{4 / 7} R^{4 / 7}} .
$$

Using prefactors obtained from our experimental data in Fig. 2.2, for an ethanol droplet of $0.9 \mathrm{~mm}$ radius, this translates to an impact velocity $U_{o}=$ $0.25 \mathrm{~m} / \mathrm{s}$.

\subsection{Conclusion}

What is the physical reason for the maximum? For higher velocities inertia dominates and flattens the droplet at impact. For lower velocities and/or smaller droplets the capillary forces try to keep the drop spherical. In between these two regimes the maximal air entrainment under the droplet is achieved.

For many applications air entrainment is undesirable and maximal wetting must be achieved. This holds for immersion lithography, wafer drying, glueing, agricultural applications, etcetera [19, 20]. Intriguingly, for inkjet drops of radius $R \sim 10 \mu \mathrm{m}$, the optimal velocity according to (2.7) is approximately $1 \mathrm{~m} / \mathrm{s}$. This lies exactly in the range at which inkjet usually operates (typically a few $\mathrm{m} / \mathrm{s}$ ), and relatively large bubbles will thus be entrapped [1]. For immersion lithography the entrapment of even micron-sized bubbles can cause practical limitations $[19,20]$. This technology is based on optical imaging of nanoscale structures, for which the optics is immersed in water to push the limits of spatial resolution. Clearly, it is crucial to avoid bubbles or to minimize their size, which also has bearing in cleaning and drying of wafers. Ideally, one should stay as far as possible from the optimal air entrainment impact velocity. Our findings will help to achieve this goal and thus optimal wetting.

\section{References}

[1] D. B. van Dam and C. Le Clerc, "Experimental study of the impact of an ink-jet printed droplet on a solid substrate", Phys. Fluids 16, 3403-3414 (2004).

[2] S. T. Thoroddsen, T. Etoh, K. Takehara, N. Ootsuka, and A. Hatsuki, "The air bubble entrapped under a drop impacting on a solid surface", J. Fluid Mech. 545, 203-212 (2005). 
[3] S. Mandre, M. Mani, and M. P. Brenner, "Precursors to splashing of liquid droplets on a solid surface", Phys. Rev. Lett. 102, 134502 (2009).

[4] M. M. Driscoll and S. R. Nagel, "Ultrafast interference imaging of air in splashing dynamics", Phys. Rev. Lett. 107, 154502 (2011).

[5] J. M. Kolinski, S. M. Rubinstein, S. Mandre, M. Mani, M. P. Brenner, D. A. Weitz, and L. Mahadevan, "Skating on a film of air: Drops impacting on a surface", Phys. Rev. Lett. 108, 074503 (2012).

[6] S. Mandre and M. P. Brenner, "The mechanism of a splash on a dry solid surface", J. Fluid Mech. 690, 148-172 (2012).

[7] L. Xu, W. W. Zhang, and S. R. Nagel, "Drop splashing on a dry smooth surface", Phys. Rev. Lett. 94, 184505 (2005).

[8] L. Xu, L. Barcos, and S. R. Nagel, "Splashing of liquids: Interplay of surface roughness with surrounding gas", Phys. Rev. E 76, 066311 (2007).

[9] R. C. A. van der Veen, T. Tran, D. Lohse, and C. Sun, "Direct measurements of air layer profiles under impacting droplets using high-speed color interferometry", Phys. Rev. E 85, 026315 (2012).

[10] M. H. W. Hendrix, R. Manica, E. Klaseboer, D. Y. C. Chan, and C. D. Ohl, "Spatiotemporal evolution of thin liquid films during impact of water bubbles on glass on a micrometer to nanometer scale", Phys. Rev. Lett. 108, 247803 (2012).

[11] M. Mani, S. Mandre, and M. P. Brenner, "Events before droplet splashing on a solid surface", J. Fluid Mech. 647, 163-185 (2010).

[12] P. D. Hicks and R. Purvis, "Air cushioning and bubble entrapment in threedimensional droplet impacts", J. Fluid Mech. 649, 135-163 (2010).

[13] P. D. Hicks and R. Purvis, "Air cushioning in droplet impacts with liquid layers and other droplets", Phys. Fluids 23, 062104 (2011).

[14] J. Eggers, M. A. Fontelos, C. Josserand, and S. Zaleski, "Drop dynamics after impact on a solid wall: theory and simulations", Phys. Fluids 22, 062101 (2010).

[15] H. N. Oguz and A. Prosperetti, "Dynamics of bubble-growth and detachment from a needle", J. Fluid Mech. 257, 111-145 (1993). 
[16] R. P. H. M. Bergmann, D. van der Meer, S. Gekle, J. van der Bos, and D. Lohse, "Controlled impact of a disk on a water surface: cavity dynamics", J. Fluid Mech. 633, 381-409 (2009).

[17] S. Gekle and J. Gordillo, "Compressible air flow through a collapsing liquid cavity", Int. J. Numer. Meth. Fluids 67, 1456âĂŞ1469 (2011).

[18] F. T. Smith, L. Li, and G. X. Wu, "Air cushioning with a lubrication/inviscid balance", J. Fluid Mech. 482, 291-318 (2003).

[19] M. Switkes, M. Rothschild, T. A. Shedd, H. B. Burnett, and M. S. Yeung, "Bubbles in immersion lithography", J. Vac. Sci. Technol. B 23, 2409-2412 (2005),

[20] K. G. Winkels, I. R. Peters, F. Evangelista, M. Riepen, A. Daerr, L. Limat, and J. H. Snoeijer, "Receding contact lines: from sliding drops to immersion lithography", EPJ - Special Topics 192, 195-205 (2011). 


\section{3 \\ Universal mechanism for air entrainment during liquid impact *}

When a mm-sized liquid drop approaches a deep liquid pool, both the interface of the drop and the pool deform before the drop touches the pool. The build-up of air pressure prior to coalescence is responsible for this deformation. Due to this deformation, air can be entrained at the bottom of the drop during the impact. We quantify the amount of entrained air numerically, using the Boundary Integral Method (BIM) for potential flow for the drop and the pool, coupled to viscous lubrication theory for the air film that has to be squeezed out during impact. We compare our results to various experimental data and find excellent agreement for the amount of air that is entrapped during impact onto a pool. Next, the impact of a rigid sphere onto a pool is numerically investigated and the air that is entrapped in this case also matches with available experimental data. In both cases of drop and sphere impact onto a pool the numerical air bubble volume $V_{b}$ is found to be in agreement with the theoretical scaling $V_{b} / V_{\text {drop }}$ sphere $\sim \mathrm{St}^{-4 / 3}$, where $\mathrm{St}$ is the Stokes number. This is the same scaling that has been found for drop impact onto a solid surface in previous research.

${ }^{*}$ Submitted as: M.H.W. Hendrix, W. Bouwhuis, D. van der Meer, D. Lohse, J.H. Snoeijer, "Universal mechanism for air entrainment during liquid impact". 


\subsection{Introduction}

The impact of a drop or a solid sphere onto a liquid pool can encompass various types of air entrainment. One possibility is that air is entrained at the top of the impacting object when the crater that is created during impact collapses, see for example Ref. [1-4]. Another type of air entrainment may occur at the bottom of the impacting object: the thin air film that is squeezed out at the impact zone is accompanied by a pressure increase that deforms the interface of the liquid before the impacting object touches the pool, which may result in air entrapment [5-9]. The early stages of deformations can be described analytically (Chapter 4). In case the impacting object is a drop, also a collection of microscopic bubbles (instead of a single entrapped bubble) may be entrapped, which can create intriguing morphologies [10]. This is also referred to as Mesler entrainment $[2,11]$. The same mechanism that is responsible for bubble entrapment at the bottom of an impacting object on a pool holds for air entrapment at the bottom of an impacting drop onto a solid [12-15]. In fact, the initial geometry of the problems is identical, see Fig. 3.1, in which the different impact scenarios and air entrapment have been depicted. We also refer to Fig. 5 of Ref. [9], in which this analogy was first proposed.

Previously, air bubble entrapment for drop impact onto a solid surface has been quantified experimentally, theoretically, and numerically [13-17]. If the effect of surface tension can be neglected we can consider the inertial regime (see Chapter 2), for which the following scaling for the entrapped air bubble volume was found:

$$
V_{b} / V_{\text {drop }} \sim \mathrm{St}^{-4 / 3}
$$

Here $V_{b} / V_{\text {drop }}$ is the air bubble volume normalized by the drop volume and St is the Stokes number which is defined as $\mathrm{St} \equiv \rho_{l} R U / \eta_{g}$, where $\rho_{l}$ is the liquid density, $R$ the drop radius, $U$ its impact velocity, and $\eta_{g}$ is the viscosity of the surrounding gas, in this case air. The Stokes number represents the competing effect of the viscous force of the draining air film and the inertial force of the liquid which ultimately determines the air bubble volume. The same scaling was found experimentally for impact of a sphere onto a pool [8], and a drop onto a pool [9]. When surface tension effects become important the scaling must be modified to include the effect of the Laplace pressure as we move towards the capillary regime, as described in Chapter 2. 
In this chapter we try to capture the mechanism of air entrapment during impact onto a deep pool numerically. We will employ a Boundary Integral method (BIM) for potential flow describing the liquid phase coupled to viscous lubrication theory for the draining microscopic air film. The advantage of using a Boundary Integral method becomes evident when the interface of the impacting object comes close to the pool and one has to resolve the microscopic air layer together with the macroscopic liquid scale. This difference in length scale can be a thousandfold for the case of a millimeter sized drop impacting onto a pool squeezing out an air film with a typical thickness of a few micrometers. In fact, the difference in length scale in the final stages of impact diverges to infinity as the drop is about to coalesce with the pool. Using a Boundary Integral method guarantees excellent interface representation, since all variables such as liquid velocity and pressure are defined at the interface.

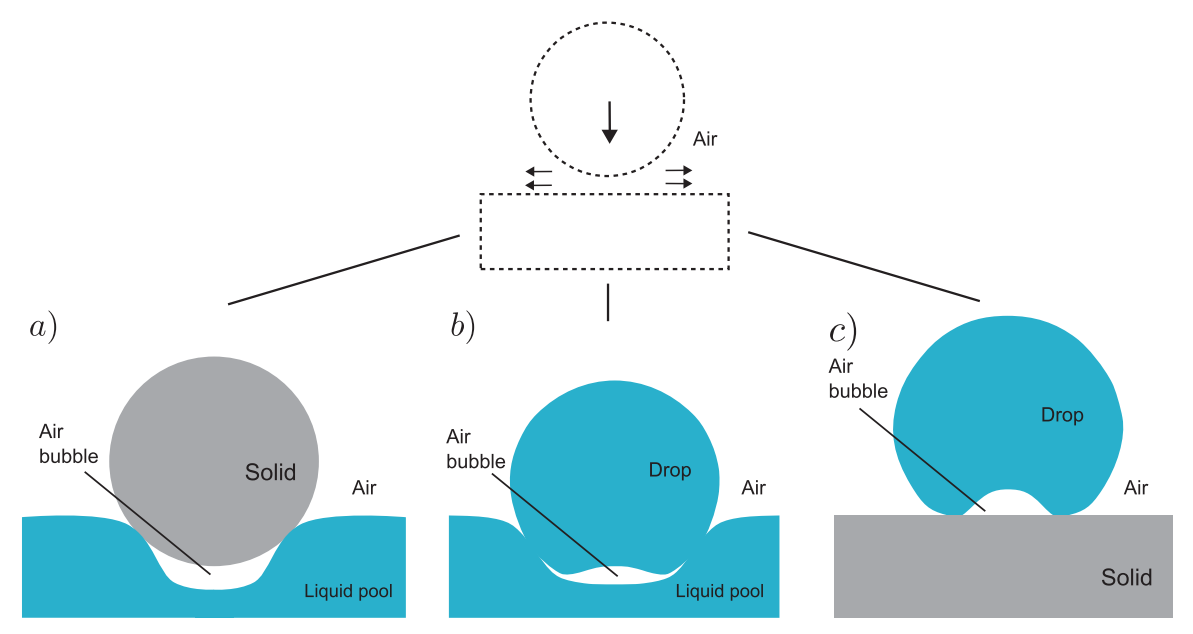

Figure 3.1: Air bubble entrapment for different impact scenarios. Bubbles and deformations are not drawn to scale. (a) Rigid sphere impact onto a pool. The pool deforms due to an increase in air pressure right under the sphere before it touches the pool, which results in an entrapped air bubble. (b) Drop impact onto a pool. Not only the pool, but also the drop consists of a deformable interface. As a result, the increased air pressure deforms both the pool and the drop and an air bubble is entrapped. (c) Drop impact onto a solid. Also here, a local increase in air pressure deforms the drop before it touches the solid and results in an entrapped air bubble. 
At the same time, the computational cost is modest, since the Boundary Integral method allows the potential problem to be solved only at the boundaries of the liquid domain: quantities in interior points can be calculated optionally as a function of the solution at the boundary. To achieve the same accurate interface representation and solving the full Navier-Stokes equations, using for example a volume-of-fluid method (see for example Ref. [18, 19]), would require a much larger computational cost.

In Sec. 3.2 we explain the theoretical framework together with the numerical method. In Sec. 3.3 we will present the results of the numerical simulations: we identify details of the pressure development in the air film and the deformation of the interfaces at the impact zone. The results of the numerical model are compared with available results regarding the entrapped bubble volume from multiple experimental works and with the scaling law (3.1). We conclude with Sec. 3.4 in which suggestions for further research are discussed.

\subsection{Theory}

\subsubsection{Dimensional analysis and numerical method}

The Reynolds number of the liquid drops we model, which is defined as $\operatorname{Re}_{l} \equiv$ $\rho_{l} R U / \eta_{l}$, is assumed to be large, $\operatorname{Re}_{l} \gg 1$. Here $\rho_{l}$ and $\eta_{l}$ are respectively the density and the dynamic viscosity of the liquid, $U$ is the impact velocity, and $R$ is the radius of drop. The flow can be regarded irrotational, that is, $\nabla \times \mathbf{u}=\mathbf{0}$. Under the additional constraint of incompressible flow inside the drop this allows the liquid dynamics to be modeled with a harmonic function $\phi$, to which the velocity field $\mathbf{u}$ is related through:

$$
\mathbf{u}=\nabla \phi
$$

The fact that the velocity potential $\phi$ obeys the Laplace equation $\nabla^{2} \phi=0$ is used to efficiently solve the potential problem, and thus the dynamics of the liquid, using the Boundary Integral Method (BIM). We use a BIM based on codes which are described in detail in Refs. [20] and [21].

While the Reynolds number of the drop is large, the Reynolds number of the thin gaseous air layer $\operatorname{Re}_{g} \equiv \rho_{g} H_{d} U / \eta_{g}$ is typically small. Here $\rho_{g}$ is the gas density and $H_{d}$ is the air film thickness in the center of the film which is referred to as the dimple height. When inserting typical parameters, $\rho_{g}$ is of order $1, H_{d}$ is of order $10^{-6}, U$ is of order 1 and $\eta_{g}$ is of order $10^{-5}$. This results in $\operatorname{Re}_{g}$ to be in the order of $10^{-1}$, which justifies the assumption. 
$(a)$

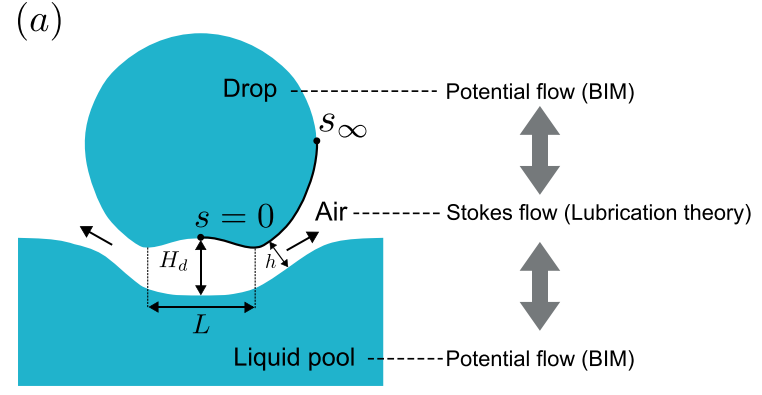

(b)

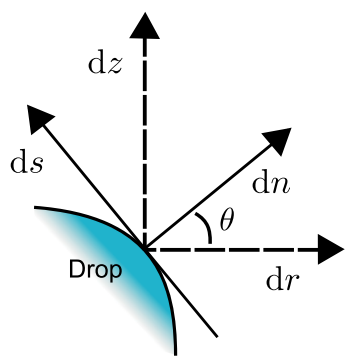

Figure 3.2: (a) Schematic of drop impact onto a a pool. The used methods are indicated in the figure: both the liquid domains are modeled with potential flow, while the air layer is described with Stokes flow. The gray arrows indicate that the flow of the air film is coupled to the dynamics of the liquid domains and vice versa. (b) Definition of the $(n, s)$-coordinate system, where $s$ is aligned along the drop curve and $n$ is the unit normal with respect to the drop.

The length scale characterizing the air layer in the lateral extension of the air film is denoted by $L$, see Fig. 3.2a. As shown in Chapter $2, H_{d} \ll L$, which, in combination with the low Reynolds number of the gas, allows the film to be described with viscous lubrication theory, see for example Ref. [22]. Note that for drops impacting with a higher speed well outside the parameter range currently considered, lubrication theory should be extended to include the effect of inertia of the gas. The dimensionless group reflecting the presence of air is the Stokes number $\mathrm{St} \equiv \rho_{l} R U / \eta_{g}$ which compares the viscous force of the air layer to the inertial force in the drop. This number is relevant for describing dimple formation, since, for high enough impact velocity $U$, this process is determined by two competing forces: the force of the viscous air layer trying to deform the drop in the center and the opposing inertial force of the drop, which must be slowed down locally in order to form a dimple. Additional dimensionless numbers incorporating surface tension $\gamma$ are the Weber number We and the capillary number Ca based on the gas properties. Summarizing, we thus have the following dimensionless parameters:

$$
\operatorname{Re}_{l} \equiv \frac{\rho_{l} R U}{\eta_{l}} \quad \operatorname{Re}_{g} \equiv \frac{\rho_{g} H_{d} U}{\eta_{g}} \quad \mathrm{St} \equiv \frac{\rho_{l} R U}{\eta_{g}} \quad \mathrm{We} \equiv \frac{\rho_{l} R U^{2}}{\gamma} \quad \mathrm{Ca} \equiv \frac{\mathrm{We}}{\mathrm{St}}
$$


The impact of a liquid drop onto a pool of the same liquid and the impact of a rigid sphere onto a liquid pool can be described with the same dimensionless numbers. As the initial geometry of the problems is identical, the difference lies in the deformability of the object, which is zero in case of the solid. The two effective control parameters that we will use here in our theoretical framework are St and We. In this work the depth of the pool is considered infinite. In case the thickness of the pool is finite, the dynamics of the pool may be altered due to the presence of a solid boundary at the bottom of the pool. For the impact onto a liquid film with finite thickness we refer the reader to Ref. [23], in which the impact onto a wetted solid is discussed.

In Fig. 3.2a an illustration of the impact of a drop onto a pool, together with the used method is shown. As is clear from this figure, the coupling between the dynamics of the air layer and the dynamics of the liquid is essential since the two liquid domains feel each other through the pressure build-up in the viscous air layer. The lubrication pressure $P_{g}$ acts on the liquid surface and appears in the unsteady Bernoulli equation which serves as a dynamic boundary condition in the BIM applied at the liquid surface:

$$
\left(\frac{\partial \phi}{\partial t}+\frac{1}{2}|\nabla \phi|^{2}\right)=-\frac{\gamma}{\rho_{l}} \kappa(s, t)-\frac{P_{g}(s, t)}{\rho_{l}}
$$

Here $P_{g}$ is the pressure in excess of the ambient pressure, due to lubrication. The curvature of the interface is represented by $\kappa(s, t)$ which is a function of the curvilinear coordinate $s$ which follows the liquid surface and time $t$. Note that unlike Chapter 2 we did not include gravitation, to make sure that the impact speed of the impacting drop stays constant during its fall. The small deformation of the pool justifies the assumption of neglecting the pressure due to hydrostatic gradients. As we have two liquid domains, two separate BI equations are solved. We take the width of the pool large enough to approach the dynamics of an infinite liquid pool. In this case a width of 4.5 times the drop radius was found to be sufficient. We focus on quantifying the amount of entrapped air by integrating the enclosed air pocket up to the moment the air layer reaches a physical minimum thickness of $0.4 \mu \mathrm{m}$. At this point the volume of the enclosed air has converged and a subsequent rupture of the air film will prevent further drainage which results in an entrapped air bubble [15]. As we focus on the dynamics just prior to rupture we can make use of an axisymmetric framework. In Ref. [7] a similar approach was used to predict the radius of the entrapped bubble which occurs when a solid sphere approaches a liquid free surface. We restrict ourselves to the inertial regime [15] for which experimental results [9] are available for a direct comparison. Since the air 
layer continually deforms and translates during the impact, lubrication equations have been developed in a moving coordinate system which is aligned with the interface of the drop. These equations will be derived in the next section.

\subsubsection{Lubrication in moving and tilted coordinate system}

In this section we develop an expression for the pressure $P_{g}$ in the air film based on lubrication theory in a moving $(n, s)$-coordinate system which is aligned along the drop surface, see the sketch in Fig. 3.2. The reason for doing this (rather than using the standard $(r, z)$-coordinate system) is that, especially for the drop onto pool impact, the moving $(n, s)$-coordinate system is not necessarily oriented as the $(r, z)$-coordinate system and therefore only the first guarantees an accurate description of the draining air film. In Appendix 3.A a case is described which shows the difference between lubrication calculated in both coordinate systems. The drop surface is taken as a reference, and the curvilinear coordinate $s$ is defined along the drop, starting at the axis of symmetry (bottom of the drop). At some large radial coordinate $s_{\infty}$ we assume atmospheric pressure. The coordinate perpendicular to $s$ is defined to be $n$. The gap thickness $h(r, t)$ is defined as the length of the perpendicular line from the drop projected onto the liquid pool. The two surfaces in the impact zone are assumed to be nearly parallel $\left(\left|\partial_{s} h\right| \ll 1\right)$, so we can apply lubrication theory in the direction along $s$.

It can be shown (see Appendix 3.B) that the continuity equation in this new $(n, s)$-coordinate system reads:

$$
\frac{u_{r}}{r}+\partial_{s} u_{s}+\partial_{n} u_{n}=0
$$

At the interface of the liquid pool $(n=h)$ we know that the fluid particles have to move with the interface. This is mathematically described with the kinematic boundary condition:

$$
\partial_{t} h+\left.\left(u_{s} \partial_{s} h\right)\right|_{n=h}=u_{n \mid n=h}-u_{n \mid n=0} .
$$

Here $\partial_{t} h$ is the time derivative of $h$. We now integrate Eq. (3.5) along the gap thickness $h$ and obtain:

$$
\int_{0}^{h} \frac{u_{r}}{r} \mathrm{~d} n+\int_{0}^{h} \partial_{s} u_{s} \mathrm{~d} n=-\int_{0}^{h} \partial_{n} u_{n} \mathrm{~d} n=u_{n \mid n=0}-u_{n \mid n=h}
$$


Using Leibniz integral rule for the second integral on the left hand side we find:

$$
\int_{0}^{h} \frac{u_{r}}{r} \mathrm{~d} n+\partial_{s} \int_{0}^{h} u_{s} \mathrm{~d} n-\left.\left(u_{s} \partial_{s} h\right)\right|_{n=h}=u_{n \mid n=0}-u_{n \mid n=h} .
$$

We now use the kinematic boundary condition formulated in Eq. (3.6) for the third term on the LHS to obtain:

$$
\int_{0}^{h} \frac{u_{r}}{r} \mathrm{~d} n+\partial_{s} \int_{0}^{h} u_{s} \mathrm{~d} n+\partial_{t} h-u_{n \mid n=h}+u_{n \mid n=0}=u_{n \mid n=0}-u_{n \mid n=h} .
$$

Canceling the terms $u_{n \mid n=h}$ and $u_{n \mid n=0}$ on both sides gives:

$$
\int_{0}^{h} \frac{u_{r}}{r} \mathrm{~d} n+\partial_{s} \int_{0}^{h} u_{s} \mathrm{~d} n+\partial_{t} h=0 .
$$

We still have to describe $u_{r}$ within the new $(s, n)$-coordinate system. Therefore we substitute $u_{r}=u_{n} \cos \theta-u_{s} \sin \theta$ in the preceding equation to get:

$$
\int_{0}^{h} \frac{1}{r} u_{n} \cos \theta \mathrm{d} n-\int_{0}^{h} \frac{1}{r} u_{s} \sin \theta \mathrm{d} n+\partial_{s} \int_{0}^{h} u_{s} \mathrm{~d} n+\partial_{t} h=0 .
$$

We assume that the main flow of the air that is squeezed out from the gap is along the $s$-coordinate, which implies that $u_{n}$ is relatively small, so we neglect the first term. The second term is an integral with respect to $n$ containing the variable $r$. This radial coordinate $r$ across $h$ is a function of $n$ : $r=n \cos \theta+c(s)$. Here $c(s)$ is the value of $r$ at the drop surface $(n=0)$ for some coordinate $s$. We thus substitute this expression for $r$ into Eq. (3.11) and neglect the first term to find:

$$
-\int_{0}^{h} \frac{\sin \theta}{n \cos \theta+c(s)} u_{s} \mathrm{~d} n+\partial_{s} \int_{0}^{h} u_{s} \mathrm{~d} n+\partial_{t} h=0 .
$$

\section{Flow profile within the air film}

As has been previously described, the Reynolds number of the thin air film is small, $\operatorname{Re}_{g} \ll 1$, and the geometry of the problem, $H_{d} \ll L$, allows us to use lubrication theory. In the $(n, s)$-coordinate system, the Stokes equations can then be reduced to: 


$$
\partial_{s} P_{g}=\eta_{g} \partial_{n}^{2} u_{s}
$$

We can integrate Eq. (3.13) twice with respect to $n$, employing a no slip boundary condition at the drop surface $\left(u_{s}=U_{d}\right.$ at $\left.n=0\right)$ as well as at the surface of the pool $\left(u_{s}=U_{p}\right.$ at $\left.n=h\right)$, to find:

$$
u_{s}=\left(\left(U_{p}-U_{d}\right) \frac{n}{h}+U_{d}\right)+\frac{1}{2 \eta_{g}} \partial_{s} P_{g}\left(n^{2}-n h\right) .
$$

The first term of Eq. (3.14) can be associated with Couette flow, caused by the movement of the interfaces. $U_{p}$ and $U_{d}$ can be extracted by differentiating the potential $\phi$ with respect to the tangential direction of the liquid surface. The second term can be associated with Poiseuille flow, which is driven by the radial pressure gradient, see also Ref. [24]. Substituting this expression for $u_{s}$ in our equation of mass conservation, Eq. (3.12), we get:

$$
\begin{array}{r}
-\int_{0}^{h} \frac{\sin \theta}{n \cos \theta+c}\left[\left(\left(U_{p}-U_{d}\right) \frac{n}{h}+U_{d}\right)+\frac{1}{2 \eta_{g}} \partial_{s}\left(n^{2}-n h\right)\right] \mathrm{d} n \\
+\partial_{s} \int_{0}^{h}\left[\left(\left(U_{p}-U_{d}\right) \frac{n}{h}+U_{d}\right)+\frac{1}{2 \eta_{g}} \partial_{s} P_{g}\left(n^{2}-n h\right)\right] \mathrm{d} n+\partial_{t} h=0 .
\end{array}
$$

In the first integral we deal with a prefactor $\sin \theta /(n \cos \theta+c)$. When taking into account the geometry of the problem we note that $n \cos \theta \ll c$. We can thus write $\sin \theta /(n \cos \theta+c) \approx \sin \theta / c$. Under this assumption, performing the integrals of Eq. (3.15) yields:

$$
\begin{array}{r}
-\frac{\sin \theta}{c}\left(\frac{h}{2}\left(U_{p}+U_{d}\right)-\frac{h^{3}}{12 \eta_{g}} \partial_{s} P_{g}\right)+\partial_{s}\left(\frac{h}{2}\left(U_{p}+U_{d}\right)-\frac{h^{3}}{12 \eta_{g}} \partial_{s} P_{g}\right) \\
+\partial_{t} h=0
\end{array}
$$

If we define $G(s) \equiv\left(\frac{h}{2}\left(U_{p}+U_{d}\right)-\frac{h^{3}}{12 \eta_{g}} \partial_{s} P_{g}\right)$ we can transform the preceding equation into a first order inhomogeneous linear ODE for $G(s)$ :

$$
\dot{G}(s)-a(s) G(s)=f(s) .
$$

Here $a(s)$ and $f(s)$ are known functions of $s$ : 


$$
\begin{aligned}
& a(s)=\frac{\sin \theta}{c(s)} \\
& f(s)=-\partial_{t} h
\end{aligned}
$$

\section{Solving the first order inhomogeneous ODE for $G(s)$}

Equation (3.17) can be solved with help of an integrating factor $I$ defined as $I(s) \equiv \mathrm{e}^{-\int a(s) \mathrm{d} s}$. Using the boundary condition $G(s)=0$ for $s=0$, because we have zero pressure gradient in the center of symmetry and zero tangential velocities, we can multiply Eq. (3.17) with $I(s)$ and solve for $G(s)$ :

$$
G(s)=\frac{1}{I(s)}\left(\int_{0}^{s} I(\tilde{s}) f(\tilde{s}) \mathrm{d} \tilde{s}\right)
$$

with $I(s)=\mathrm{e}^{-\int_{0}^{s} a(\tilde{s}) \mathrm{d} \tilde{s}}$.

We can now substitute $G(s) \equiv\left(\frac{h}{2}\left(U_{p}+U_{d}\right)-\frac{h^{3}}{12 \eta_{g}} \partial_{s} P_{g}\right)$ back into Eq. (3.20) to obtain an equation for $\partial_{s} P_{g}$ :

$$
\partial_{s} P_{g}=-\frac{12 \eta_{g}}{h^{3}}\left(\frac{1}{I(s)}\left(\int_{0}^{s} I(\tilde{s}) f(\tilde{s}) \mathrm{d} \tilde{s}\right)-\frac{h}{2}\left(U_{p}+U_{d}\right)\right)
$$

We note that we have to evaluate two numerical integrals to calculate $\partial_{s} P_{g}$. In order to find the pressure $P_{g}(s)$ we integrate Eq. (3.21) using atmospheric pressure for some large value for $s_{\infty}$ well outside the thin air gap as a boundary value.

As a check of our analysis we now orientate the $(n, s)$-coordinate system in such away that $s=r$, to recover the lubrication equation in the conventional $(r, z)$-coordinate system. In that case we have $\theta=-\pi / 2$, and we can write for $a(s)$ :

$$
a(s=r)=\frac{\sin \theta}{r}=-\frac{1}{r}
$$

The integrating factor $I$ now becomes:

$$
I(s=r)=\mathrm{e}^{-\int_{0}^{r} a(\tilde{r}) d \tilde{r}}=e^{\ln r}=r
$$

Substituting Eq. (3.23) into Eq. (3.21) and using the proposition $s=r$ and setting $U_{b}=0$ and $U_{d}=0$, we can now write Eq. (3.21) as: 


$$
\begin{aligned}
\partial_{r} P_{g} & =-\frac{12 \eta_{g}}{h^{3}}\left(\frac{1}{I(r)}\left(\int_{0}^{r} I(\tilde{r}) f(\tilde{r}) \mathrm{d} \tilde{r}\right)-\frac{h}{2}\left(U_{b}+U_{d}\right)\right) \\
& =\frac{12 \eta_{g}}{h^{3}}\left(\frac{1}{r}\left(\int_{0}^{r} \tilde{r} \partial_{t} h \mathrm{~d} \tilde{r}\right)\right) .
\end{aligned}
$$

We inspect that Eq. (3.24) is the equation for the radial pressure gradient for viscous lubrication theory in the conventional $(r, z)$-coordinate system [25], which gives a consistency check for our analysis. This was also numerically verified.

\section{$3.3 \quad$ Results}

In this section simulation results will be discussed, starting with Sec. 3.3.1 in which we treat the drop impact onto a pool. The interface deformations and pressure development in the viscous air layer are quantified. In Sec. 3.3.2 we will focus on rigid sphere impact onto a pool. For both impact scenarios we quantify the size of the air bubble that is entrapped and directly compare with various experimental results $[8,9,15]$. In Sec. 3.3.3 we will compare the dynamics of both impact scenarios and identify symmetrical behavior.

\subsubsection{Drop impact onto a pool}

Figure 3.3a displays a typical result for drop impact onto a pool. The results are expressed in dimensional form to match the experimental conditions of the work of Ref. [9], to which the numerical results in this work will be compared. In the first frame corresponding to $t=0 \mathrm{~ms}$ the initial condition of the simulation at the impact zone is shown. An initial separation of $h_{0}=50 \mu \mathrm{m}$ is used. Convergence tests regarding the initial release height have been conducted, and an initial separation of $h_{0}=50 \mu \mathrm{m}$ was found to be appropriate for the lubrication pressure to be still negligible at this distance for the parameter range which is of interest in this study. At $t=0.12 \mathrm{~ms}$ it can be seen that the pool and the drop experience the increased air pressure and thus the interfaces deform. In the lower panel of this frame, the increase in pressure is indeed visible. At $t=0.15 \mathrm{~ms}$ the drop is getting closer to the pool, and the interfaces have been further deformed. It can also be noted that the pressure maximum corresponds to a location where the separation between the drop and the pool is smallest. The location of smallest separation is now not located in the center at $r=0$ anymore. This behavior is typical for impact 
(a)
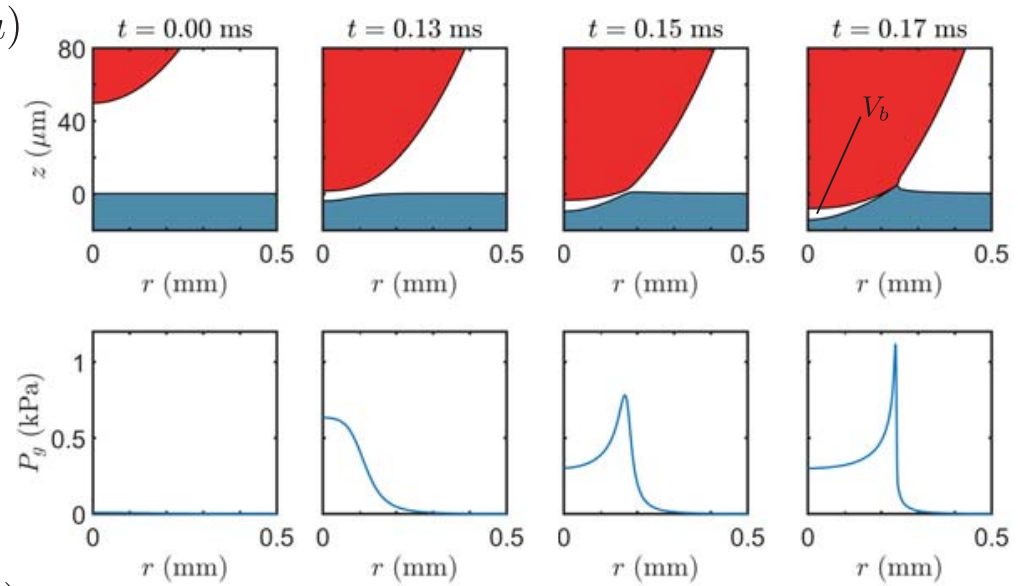

(b)

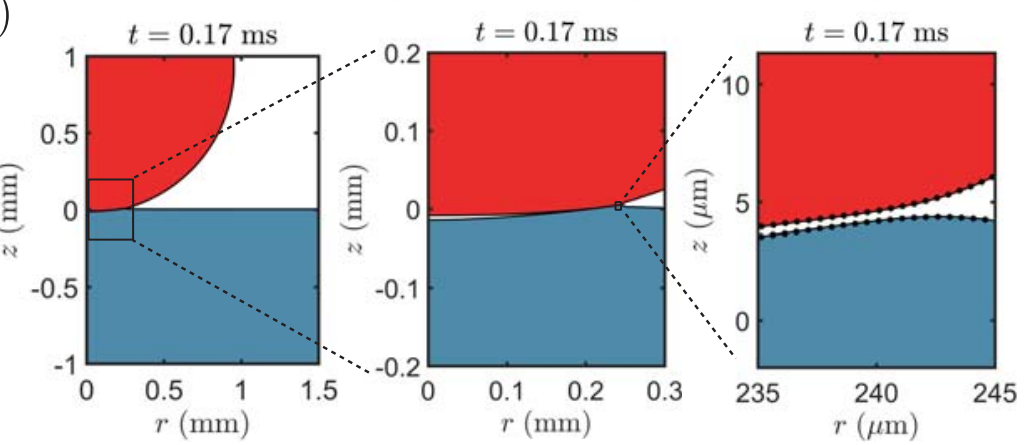

Figure 3.3: (a) Drop impact onto a liquid pool. Note the different length scales for the $r$-axis and $z$-axis in the shape plots. The impact speed is $U=0.42 \mathrm{~m} / \mathrm{s}$ and the drop radius is $R=0.95 \mathrm{~mm}$. The density and surface tension of the liquid are respectively $\rho=916 \mathrm{~kg} / \mathrm{m}^{3}$ and $\gamma=0.020 \mathrm{~N} / \mathrm{m}$. These impact parameters correspond to $\mathrm{St}=2.0 \times 10^{4}$ and $\mathrm{We}=7.7$. The simulation starts at time $t=0 \mathrm{~ms}$ at a separation of $h_{r=0}=50 \mu \mathrm{m}$. Due to the approach of the sphere, the excess air pressure $P_{g}$ will increase and acts on both the drop and the liquid pool $(t=0.13 \mathrm{~ms})$. At the final stage $(t=0.17 \mathrm{~ms})$ the minimum separation of the interfaces reaches $0.4 \mu \mathrm{m}$ and the simulation is stopped. The bubble volume $V_{b}$ can thus be determined. (b) Part of the simulation domain with detailed snapshots of the air film at $t=0.17 \mathrm{~ms}$. In the third snapshot the actual node distribution around the smallest separation point can be inspected. This is the most refined distribution of computational nodes that is used. For the region outside the gap a coarser node distribution is sufficient. 
events involving a free surface and has been experimentally observed for e.g. drop impact onto a pool [9, 10], drop impact onto a solid surface [26], sphere impact onto a pool [8] and bubble impact onto a wall in a liquid tank [27]. In the final frame $t=0.17 \mathrm{~ms}$ we observe that the two interfaces are very close together having a minimum separation of $0.4 \mu \mathrm{m}$.

We note that the interfaces up to the final stage of impact are very well resolved, see Fig. 3.3b in which the final frame at $t=0.17 \mathrm{~ms}$ is shown on various scales while keeping both axes the same length scale. In the first frame of this panel a macroscopic view of the simulation domain is shown. In the second frame the impact zone is selected and magnified. The slender geometry of the microscopic air film can be noted. In the third frame the region of closest separation is magnified. Indeed, the interfaces are very close together; the minimum separation is $0.4 \mu \mathrm{m}$. The computational nodes used for discretization of the surface are also shown in this final frame. An adaptive grid on the fluid surface allows for local refinement at the region of closest separation which results in the total number of nodes to be only of order 100, while capturing both the microscopic dynamics at the impact zone and the large scale motion of the millimeter sized drop.

We further note from the final frame in Fig. 3.3a that a microscopic air film finds itself trapped between the drop and the pool. It is this entrapped air that constitutes the air bubble that is dragged into the liquid when the air film ruptures at the thinnest point and, thereafter, breaks the axisymmetry of the problem. In this work we do not attempt to simulate the complex rupture process of the air film itself, which is ultimately determined by surface chemistry, see for example Ref. [28]. Instead, we focus on the dynamics up to the rupture point, which is taken to happen at a rupture thickness of $0.4 \mu \mathrm{m}$. At this point, the volume of the entrained air has converged and can thus be determined, see the final frame of Fig. 3.3a. This procedure is in line with previous research [15], where experimentally the volume of the air pocket just before rupture was indeed found identical to the volume of the entrapped bubble. In Ref. [10] the final thickness of the air film is estimated to be on the order of $\sim 0.2 \mu \mathrm{m}$. The final thickness that is reached during an experiment depends on the type of fluid and is sensitive to experimental conditions, e.g., how clean the fluid is [28]. Here we choose to stick to a minimum thickness consistent with Chapter 2, which is $0.4 \mu \mathrm{m}$. In Appendix 3.C a figure is included which shows that the volume of the air bubble that can be identified numerically has indeed converged for a minimum thickness of $0.4 \mu \mathrm{m}$, by also including the bubble volumes calculated for a minimum thickness of $0.2 \mu \mathrm{m}$. 


\subsubsection{Rigid sphere impact onto a pool}

The impact of a sphere onto a pool prior to coalescence is similar to the case of a drop impacting on a pool, except that in the case of an impacting sphere the deformability of the impacting object is zero. This scenario has been simulated by making an undeformable sphere approach the pool. The same equations are solved as described in Sec. 3.2, except for the fact that no BIM is needed for the impacting sphere since the interface of the sphere is fixed. The result is depicted in Fig. 3.4. Just as in the case of drop impact onto a pool, a microscopic air bubble is entrapped. As can be inspected, the air bubble has a similar shape, but its size is smaller than in case a drop impacts onto the pool (with the same impact parameters), as can be inferred from a comparison to Fig. 3.3a.

The size of the air bubble can be quantified from the numerical simulation and is compared for both drop impact and sphere impact onto a pool with various experimental results in Fig. 3.5a. We see that the numerical results of both drop and sphere impact onto a pool are in quantitative agreement with experimental work. We deduce from the numerical results that the air bubble
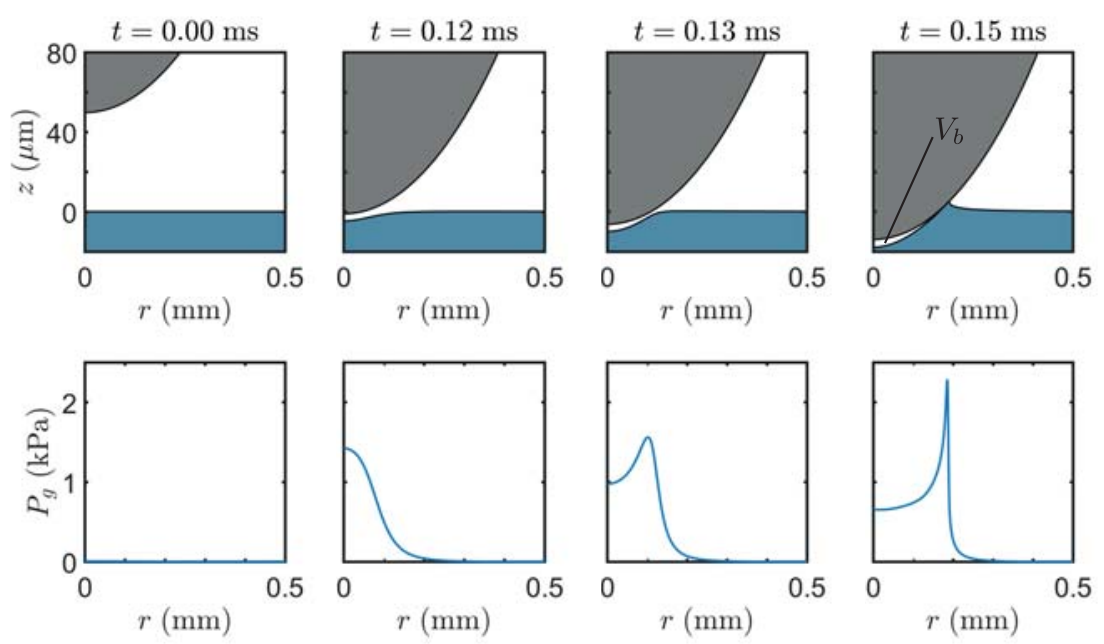

Figure 3.4: Rigid sphere impact onto a pool. The impact speed is $U=$ $0.42 \mathrm{~m} / \mathrm{s}$ and the radius is $R=0.95 \mathrm{~mm}$. The density and surface tension of the liquid are respectively $\rho=916 \mathrm{~kg} / \mathrm{m}^{3}$ and $\gamma=0.020 \mathrm{~N} / \mathrm{m}$. These impact parameters correspond to $\mathrm{St}=2.0 \times 10^{4}$ and $\mathrm{We}=7.7$. 
volume is indeed larger when a drop instead of a sphere impacts onto a pool for all St, which is supported by experiments of Ref. [9]. Furthermore, we observe that numerical results are in agreement with the scaling law presented in Eq. (3.1), $V_{b} / V_{\text {drop }} \sim \mathrm{St}^{-4 / 3}$. As experiments have shown, in this regime, the viscosity of the liquid is not important for the final bubble volume that is entrapped, see Ref. $[8,9]$, which is again confirmed by the current modeling technique which captures the essential physics determining the air bubble volume: a potential flow calculation that does not involve liquid viscosity coupled to viscous lubrication theory for the intervening air layer.

\subsubsection{Deformations of interfaces: symmetrical behavior}

We will now further investigate the fact that the bubble volume for drop impact onto a pool is larger compared to the case where we deal with only one deformable interface during impact as is the case with rigid sphere impact onto a pool. In Fig. 3.6 a closer inspection of the drop impact onto a pool is depicted. In this figure we track the relative deformation of both the pool and the drop, denoted by $\delta_{\text {drop }}$ and $\delta_{\text {pool }}$ respectively. Here $\delta_{\text {drop }}$ is defined as the deformation of the drop relative to an undeformed sphere impacting with constant speed $U$ and $\delta_{\text {pool }}$ is defined as the deformation of the pool relative to the horizon $z=0$. Interestingly, both interface deformations behave identically. One may indeed expect that two deformable interfaces which react similar to an external pressure deform in an identical way, but note that the upper domain (drop) and the lower domain (pool) do not have the same unperturbed geometry, owing to the radius of curvature of the drop. Since both media respond identically to the pressure pulse, the weak curvature with respect to the width of the localized pressure has a negligible influence: on the scale of the pressure pulse, both domains are essentially flat. We therefore expect to recover a symmetric response in the upper and lower domains. To illustrate this further, we compute the kinetic energy and the velocity inside the drop and the pool using a technique described in Ref. [29] to evaluate quantities close to the interface which need special attention as the singular behavior of the Green's function in the Boundary Integral equation becomes apparent for these points. Figure 3.7a shows the result in the frame of the pool. To highlight the symmetry, we also evaluate these quantities in a frame moving at a speed $U / 2$ in an upward direction, which results in a frame of reference in which both the drop and pool move with a speed $U / 2$ towards each other. Indeed, the velocity fields and kinetic energies are now identically distributed, see Fig. 3.7b. 
(a)

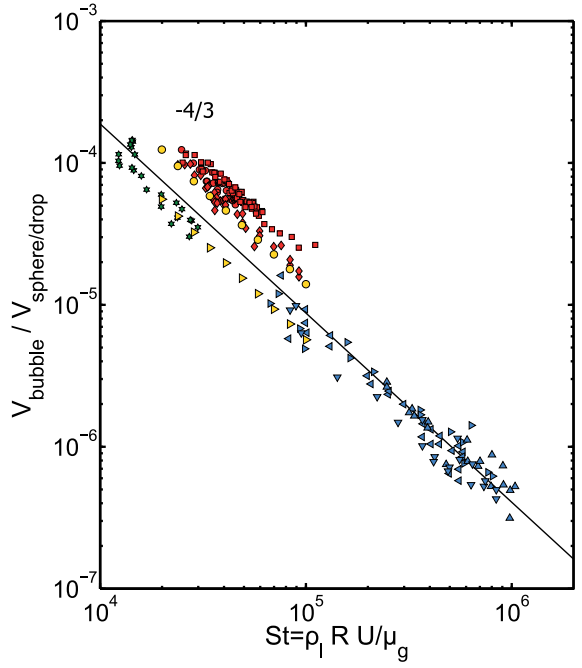

$$
n=1
$$

$n=1$ BIM, rigid sphere on pool

4 $15 \mathrm{~mm}$ steel sphere on water (Marston et al., 2011)

- $20 \mathrm{~mm}$ steel sphere on 10cSt oil (Marston et al., 2011)

$\Delta 20 \mathrm{~mm}$ steel sphere on $25 \mathrm{cSt}$ oil (Marston et al., 2011)

- Ethan steel sphere on 1cSt SDS (Marston et al., 2011) (b)

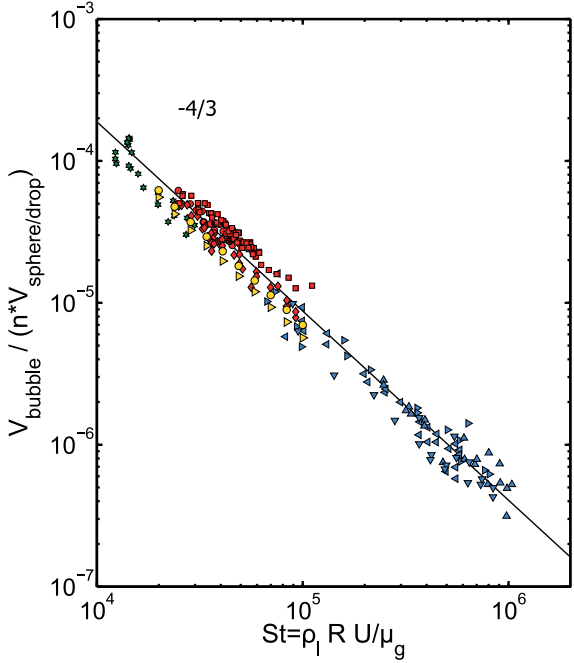

$n=2$

- BIM, drop on pool

Drop on pool, silicone oil (5cSt) (Tran et al. 2013)

Drop on pool, silicone oil (10cSt) (Tran et al. 2013)

- Drop on pool, silicone oil (20cSt) (Tran et al. 2013)

Figure 3.5: Figure adapted from Ref. [9]. BIM results are superimposed in yellow symbols. (a) Various experimental data for the normalized bubble volume $V_{b} / V_{\text {sphere }}$ drop are shown. Excellent quantitative agreement was found with numerical results. (b) The data, both numerical and experimental, was found to collapse on one single curve by normalizing $V_{b}$ as $V_{b} /\left(n V_{\text {sphere }}\right.$ drop $)$ with $n$ the number of free interfaces involved during impact. This number is 2 instead of 1 in case of drop impact onto a liquid pool. 

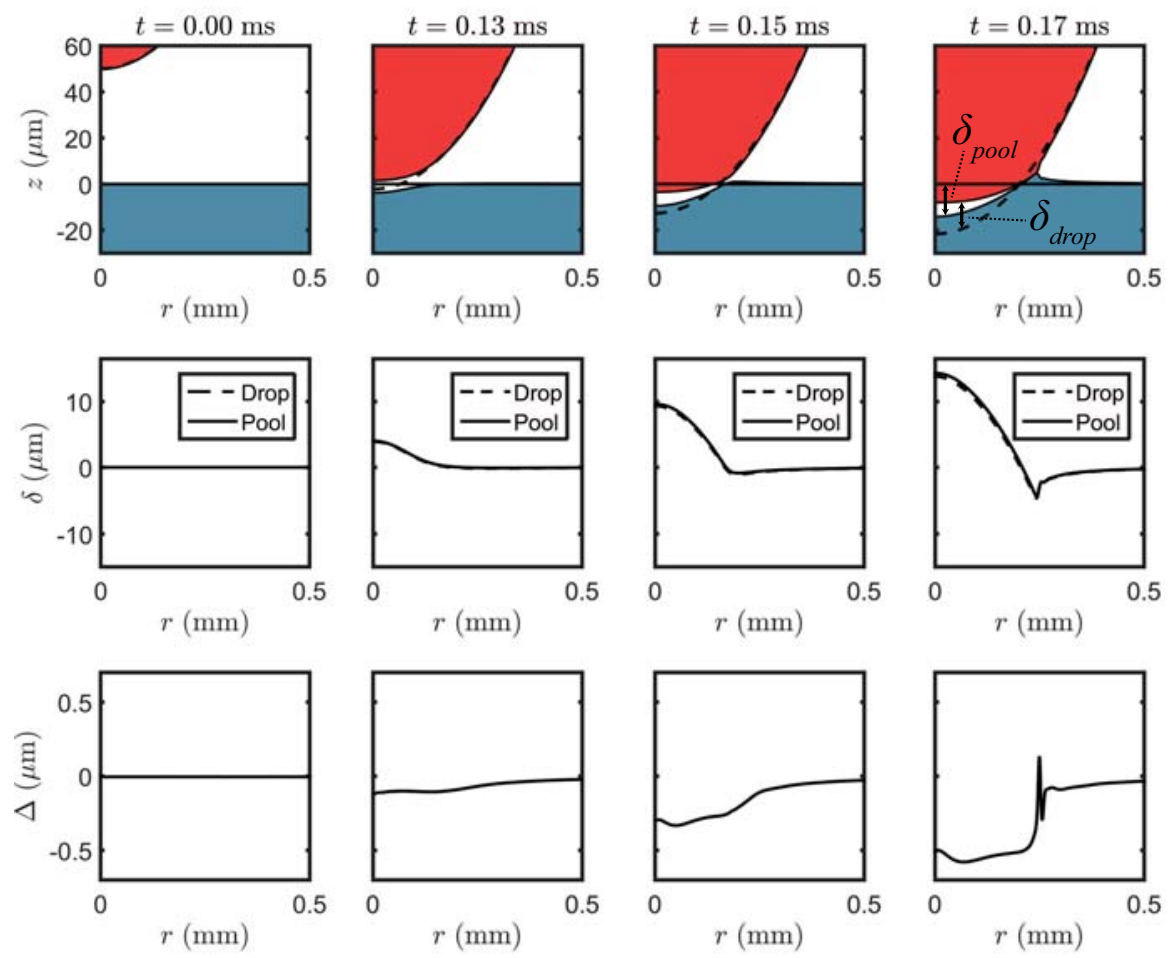

Figure 3.6: Drop impact onto a pool with a corresponding plot of the relative deformation $\delta$ of both the pool and the drop. In the final frame of the upper panel the definition of $\delta$ is shown. We observe excellent overlap between the relative deformations, which is emphasized in the lower panel where $\Delta=$ $\delta_{\text {drop }}-\delta_{\text {pool }}$, the difference between the two relative deformations, is shown. The same impact conditions as for the case described in Fig. 3.3 are used: The impact speed is $U=0.42 \mathrm{~m} / \mathrm{s}$ and the radius is $R=0.95 \mathrm{~mm}$. The density and surface tension of the liquid are respectively $\rho=916 \mathrm{~kg} / \mathrm{m}^{3}$ and $\gamma=0.020 \mathrm{~N} / \mathrm{m}$, which corresponds to $\mathrm{St}=2.0 \times 10^{4}$ and $\mathrm{We}=7.7$. 
(a)
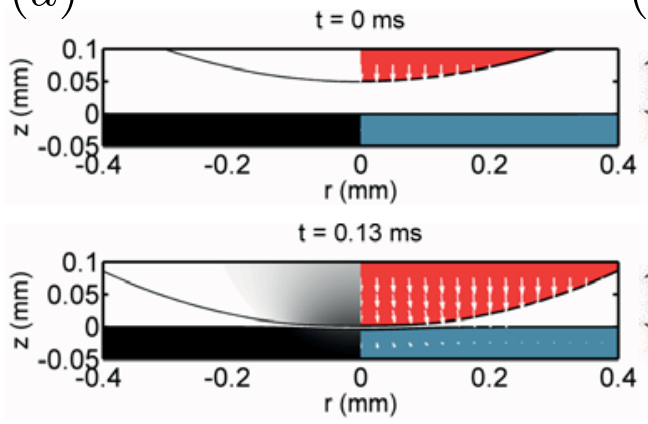

$\mathrm{t}=0.15 \mathrm{~ms}$

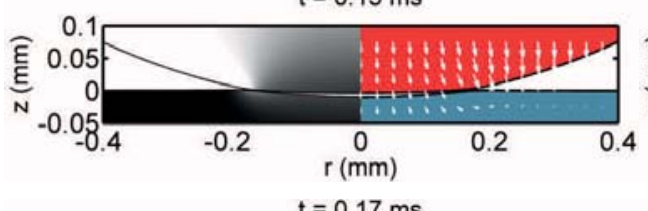

$\mathrm{t}=0.17 \mathrm{~ms}$

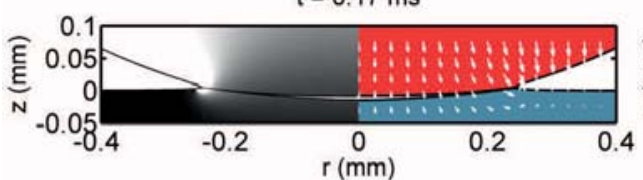

(b)

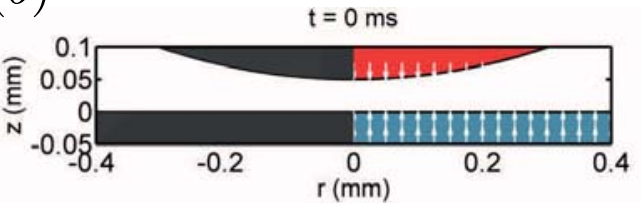

$\mathrm{t}=0.13 \mathrm{~ms}$

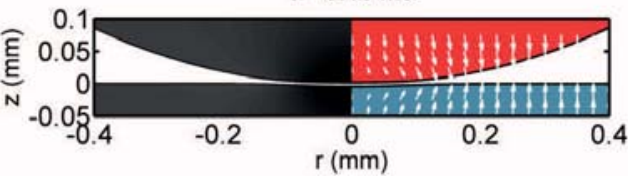

$\mathrm{t}=0.15 \mathrm{~ms}$

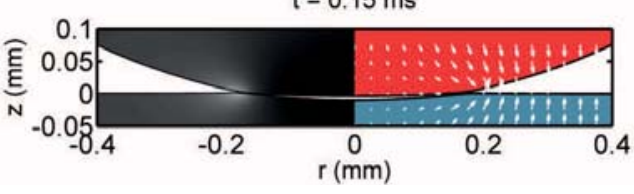

$\mathrm{t}=0.17 \mathrm{~ms}$

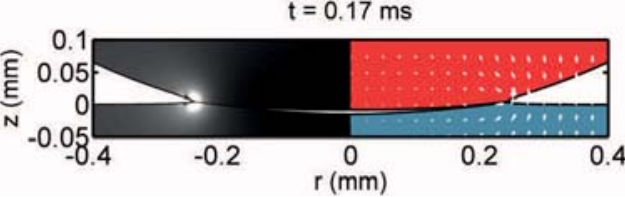

Figure 3.7: Kinetic energy monitoring during drop impact onto a pool, with impact parameters as described in Fig. 3.3. (a) In the left half, the kinetic energy $K$ is gray scale coded: black is zero kinetic energy, white is maximum kinetic energy in the system which is $K=\frac{1}{2} \rho U^{2}$. (b) The kinetic energy is recalculated in a moving reference frame moving upwards at $\frac{1}{2} U$. This results in a frame of reference in which both the pool and drop move with a speed of $\frac{1}{2} U$ towards each other. Again the left half of the figure shows the kinetic energy. We observe a symmetric behavior which supports the hypothesis that the pool and drop react in a symmetric way to the local pressure increase. 
This implies that there will be a bigger entrapped air bubble as compared to the case where only one of the interfaces is able to deform. To quantify this hypothesis we compare the bubble sizes of drop and sphere impact onto a pool and find a factor 2 difference, see Fig. 3.5b. Here half the air bubble volume of drop impact onto a pool was found to collapse onto the experimental and numerical results incorporating only one deformable interface, i.e. the sphere impact onto a pool, but also the drop impact onto a solid. Ref. [9] contained another approach to collapse the data of bubble volumes of drop impact onto a pool by correcting the corresponding impact St number by a factor 2, which also collapses the data. In this present work it is shown that an approach based on considering the number of deformable interfaces (either 1 or 2) can also serve to obtain a unifying view on the air bubble entrapment.

\subsection{Conclusion}

In this work air entrapment during liquid drop and rigid sphere impact onto a deep liquid pool has been numerically investigated using a Boundary Integral Method (BIM) for potential flow for the liquid phase coupled to the viscous lubrication approximation for the subphase air which is squeezed out during impact. Excellent agreement with experimental work was found when comparing the amount of air that is entrained during impact. When considering drop impact onto a pool both liquid interfaces were found to deform identically relative to their undeformed shape. This leads to an explanation as to why bubble volumes in case of drop impact onto a pool were found to be exactly twice the size of those that are found, both experimentally and numerically, in impacts events involving only one deformable interface, that is, rigid sphere impact onto a pool and drop impact onto a solid.

In this study (inertial and) compressibility effects of the air have been neglected. It can be expected that at higher impact velocity the compressibility of the intervening air will become important, see for example Ref. [17]. In addition, the current modeling technique is limited to an axisymmetric 2D framework. To account for 3D impact problems, for which experimental data starts to emerge [30], the modeling technique needs to be extended to 3D. With a 3D model also oblique collisions can be investigated. Furthermore, the crossover towards the capillary regime (see Chapter 2) is not within the scope of the present work. Herein lies a possibility for future research. 


\section{A Comparison $(r, z)$-system with $(n, s)$-system}

The advantage of the use of the moving $(n, s)$-coordinate system to calculate the lubrication pressure becomes evident when the interfaces start to deform and the thin air film is not aligned with the $(r, z)$-coordinate system anymore. To illustrate this, we selected a case for drop impact onto a pool as shown and described in Fig. 3.8. In Fig. 3.8a we inspect that the two different lubrication methods yield similar results from a macroscopic point of view. But when focusing on the film thickness $h$ between the two interfaces, shown in Fig. 3.8b, the difference between the two methods becomes apparent. We note that in the first stages of impact (up to $t=0.50 \mathrm{~ms}$ ) the air film is still aligned with the $(r, z)$-coordinate system, and the two lubrication methods yield similar results. But in the final stages of impact the drop sinks deeper into the pool and the air film is not aligned anymore with the $(r, z)$-coordinate system. The $(r, z)$-coordinate system therefore is now no longer adequate, which reflects in the thinning behavior which starts to differ from the results obtained with the $(n, s)$-coordinate system that moves with the drop. In the final stage $(t=3.00 \mathrm{~ms})$ the conventional $(r, z)$-coordinate system clearly shows a wrong film profile, as the minimum gap thickness is now located at the center $r=0$, while it should be located off center as shown by many experiments $[7,9$, 10, 26]. This underpins the need for an appropriate moving $(n, s)$-coordinate system, from which the conventional $(r, z)$-coordinate system is a special case which is recovered when the lubrication gap is aligned with the $(r, z)$-axes. While upfront it was not clear whether the interfaces would deform to such an extent that a moving coordinate system was needed, the generalized coordinate system guarantees accurate lubrication calculations in any scenario.

\section{B Continuity in curvilinear coordinates}

To derive Eq. (3.5) in a $(n, s)$-coordinate system that moves along with the drop surface (see Fig. 3.2) we start from the continuity equation in axisymmetric $(r, z)$-coordinates:

$$
\frac{u_{r}}{r}+\frac{\partial u_{r}}{\partial r}+\frac{\partial u_{z}}{\partial z}=0
$$

We now want to write the last two terms of the LHS of Eq. (3.25) in terms of the $(n, s)$-coordinate system, that is: 

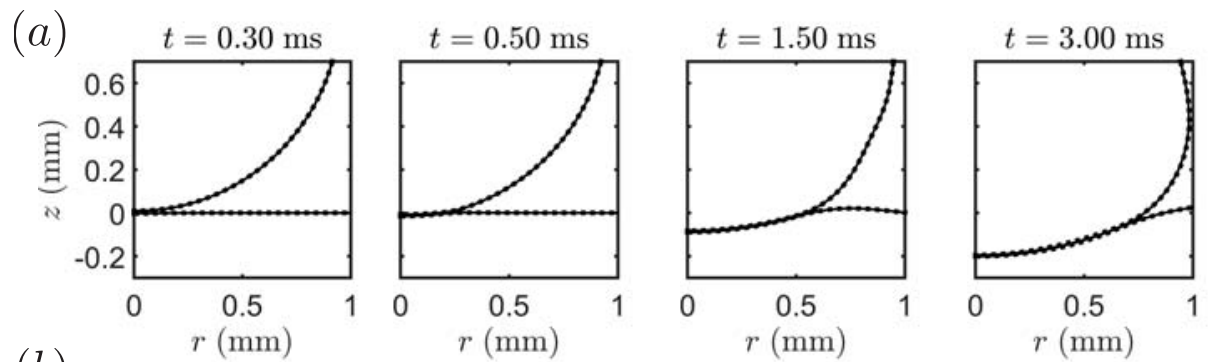

(b)
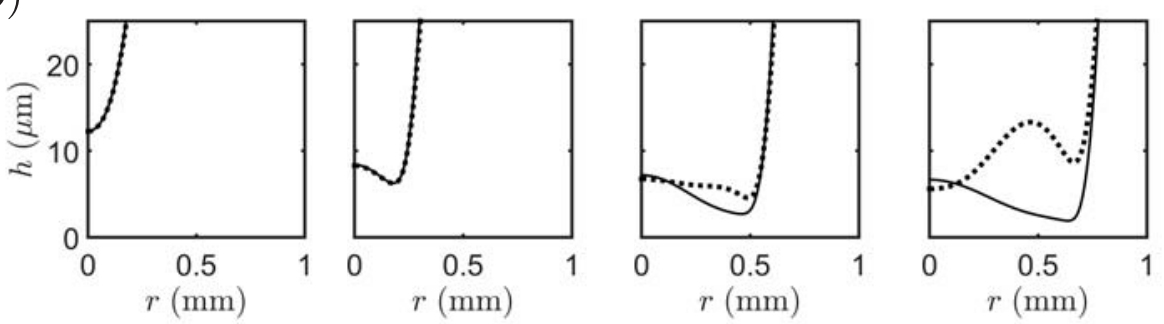

Figure 3.8: Drop impact onto a liquid pool. The impact speed is $U=0.14 \mathrm{~m} / \mathrm{s}$ and the drop radius is $R=0.95 \mathrm{~mm}$. The density and surface tension of the liquid are respectively $\rho=916 \mathrm{~kg} / \mathrm{m}^{3}$ and $\gamma=0.020 \mathrm{~N} / \mathrm{m}$. These impact parameters correspond to $\mathrm{St}=6.7 \times 10^{3}$ and $\mathrm{We}=0.85$. (a) The macroscopic drop shape together with the pool shape is shown. The solid line corresponds to calculations done in the moving $(n, s)$-coordinate system. The dashed line shows the result for the same case with the only difference that lubrication is now calculated in a conventional $(r, z)$-coordinate system. (b) To illustrate the different behavior of the two approaches the corresponding film thicknesses are shown (note the smaller length scale of the vertical axes). It can be seen that in the final stage of impact the draining of the film behaves differently. 


$$
\frac{u_{r}}{r}+\frac{\partial u_{r}}{\partial r}+\frac{\partial u_{z}}{\partial z}=\frac{u_{r}}{r}+\left(\frac{\partial u_{r}}{\partial n} \frac{\partial n}{\partial r}+\frac{\partial u_{r}}{\partial s} \frac{\partial s}{\partial r}\right)+\left(\frac{\partial u_{z}}{\partial n} \frac{\partial n}{\partial z}+\frac{\partial u_{z}}{\partial s} \frac{\partial s}{\partial z}\right)
$$

The two coordinate systems are related as follows (see also Fig. 3.2):

$$
\begin{array}{r}
\mathrm{d} s=-\mathrm{d} r \sin \theta+\mathrm{d} z \cos \theta \\
\mathrm{d} n=\mathrm{d} r \cos \theta+\mathrm{d} z \sin \theta
\end{array}
$$

Using the preceding relation we can write Eq. (3.26) as:

$$
\frac{u_{r}}{r}+\frac{\partial u_{r}}{\partial r}+\frac{\partial u_{z}}{\partial z}=\frac{u_{r}}{r}+\left(\frac{\partial u_{r}}{\partial n} \cos \theta-\frac{\partial u_{r}}{\partial s} \sin \theta\right)+\left(\frac{\partial u_{z}}{\partial n} \sin \theta+\frac{\partial u_{z}}{\partial s} \cos \theta\right)
$$

We now have to express $u_{r}$ and $u_{z}$ as function of $(s, n)$, that is:

$$
\begin{aligned}
& u_{r}(n, s)=u_{n}(n, s) \cos \theta-u_{s}(n, s) \sin \theta \\
& u_{z}(n, s)=u_{n}(n, s) \sin \theta+u_{s}(n, s) \cos \theta
\end{aligned}
$$

Substituting the above expressions for $u_{r}$ and $u_{z}$ into Eq. (3.29) and simplifying we find:

$$
\frac{u_{r}}{r}+\frac{\partial u_{r}}{\partial r}+\frac{\partial u_{z}}{\partial z}=\frac{u_{r}}{r}+\frac{\partial u_{n}}{\partial n}+\frac{\partial u_{s}}{\partial s}
$$

\section{C Sensitivity of the entrapped bubble volume to the rupture thickness}

The physics of the rupture of the air film, which is dependent on the surface chemistry of the impacting object and the pool, is not captured in the numerical model. As a result, a minimum thickness at which the film ruptures has to be imposed, which is referred to as the rupture thickness. In our work the rupture thickness is taken to be $0.4 \mu \mathrm{m}$ (consistent with Chapter 2). In Fig. 3.9 it is shown that the volume of the air bubble has already converged for this value of the rupture thickness. Taking the rupture thickness twice as small, $0.2 \mu \mathrm{m}$, does not significantly change the amount of air trapped. We thus conclude that a minimal rupture thickness of $0.4 \mu \mathrm{m}$ suffices to describe the results. 


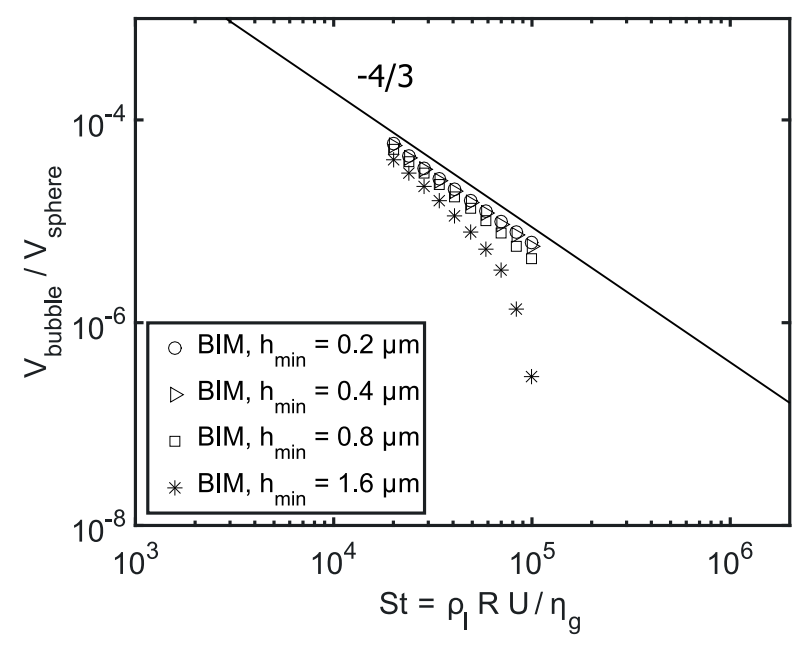

Figure 3.9: Sphere impact onto a pool, with impact parameters as described in Fig. 3.4. The bubble volume calculated for different rupture thicknesses $h_{\min }$ is shown. At a rupture thickness $h_{\min }$ of $0.4 \mu \mathrm{m}$ the air bubble volume is converged, as taking the rupture thickness twice as small to a value of $0.2 \mu \mathrm{m}$ does not significantly alter the result.

\section{References}

[1] H. N. Oguz and A. Prosperetti, "Bubble entrainment by the impact of drops on liquid surfaces", J. Fluid Mech. 219, 143-179 (1990).

[2] H. C. Pumphrey and P. A. Elmore, "Entrainment of bubbles by drop impacts", J. Fluid Mech. 220, 539-567 (1990).

[3] A. Wang, C. Kuan, and P.-H. Tsai, "Do we understand the bubble formation by a single drop impacting upon liquid surface?", Phys. Fluids 25, 101702 (2013).

[4] S. Chen and L. Guo, "Viscosity effect on regular bubble entrapment during drop impact into a deep pool", Chem. Eng. Sci. 109, 1-16 (2014).

[5] S. T. Thoroddsen, T. G. Etoh, and K. Takehara, "Air entrapment under an impacting drop", J. Fluid Mech. 125-134 (2003). 
[6] S. T. Thoroddsen, T. G. Etoh, K. Takehara, N. Ootsuka, and Y. Hatsuki, "The air bubble entrapped under a drop impacting on a solid surface", Journal of Fluid Mechanics 545, 203-212 (2005).

[7] P. D. Hicks, E. V. Ermanyuk, N. V. Gavrilov, and R. Purvis, "Air trapping at impact of a rigid sphere onto a liquid", J. Fluid Mech. 695, 310-320 (2012).

[8] J. O. Marston, I. U. Vakarelski, and S. T. Thoroddsen, "Bubble entrapment during sphere impact onto quiescent liquid surfaces", J. Fluid Mech. 680, 660-670 (2011).

[9] T. Tran, H. de Maleprade, C. Sun, and D. Lohse, "Air entrainment during impact of droplets on liquid surfaces", J. Fluid Mech. 726, R3 (2013).

[10] S. T. Thoroddsen, M.-J. Thoraval, K. Takehara, and T. G. Etoh, "Microbubble morphologies following drop impacts onto a pool surface", J. Fluid Mech. 708, 469-479 (2012).

[11] L. Esmailizadeh and R. Mesler, "Bubble entrainment with drops", J. Coll. Int. Sci. 110, 561-574 (1986).

[12] D. B. van Dam and C. Le Clerc, "Experimental study of the impact of an ink-jet printed droplet on a solid substrate", Phys. Fluids 16, 3403-3414 (2004).

[13] M. Mani, S. Mandre, and M. P. Brenner, "Events before droplet splashing on a solid surface", J. Fluid Mech. 647, 163-185 (2010).

[14] P. D. Hicks and R. Purvis, "Air cushioning and bubble entrapment in three-dimensional droplet impacts", J. Fluid Mech. 649, 135-163 (2010).

[15] W. Bouwhuis, R. C. A. Van Der Veen, T. Tran, D. L. Keij, K. G. Winkels, I. R. Peters, D. Van Der Meer, C. Sun, J. H. Snoeijer, and D. Lohse, "Maximal air bubble entrainment at liquid-drop impact", Phys. Rev. Lett. 109, 264501 (2012), See Chapter 2 of this thesis.

[16] S. Mandre, M. Mani, and M. P. Brenner, "Precursors to splashing of liquid droplets on a solid surface", Phys. Rev. Lett. 102, 134502 (2009).

[17] P. D. Hicks and R. Purvis, "Air cushioning in droplet impacts with liquid layers and other droplets", Phys. Fluids 23, 062104 (2011). 
[18] M.-J. Thoraval, K. Takehara, T. G. Etoh, S. Popinet, P. Ray, C. Josserand, S. Zaleski, and S. T. Thoroddsen, "von kármán vortex street within an impacting drop", Phys. Rev. Lett. 108, 264506 (2012).

[19] Y. Guo, L. Wei, G. Liang, and S. Shen, "Simulation of droplet impact on liquid film with clsvof", Int. Commun. Heat Mass Trans. 53, 26-33 (2014).

[20] H. N. Oguz and A. Prosperetti, "Dynamics of bubble growth and detachment from a needle", Journal of Fluid Mechanics 257, 111-145 (1993).

[21] S. Gekle and J. M. Gordillo, "Compressible air flow through a collapsing liquid cavity", International Journal for Numerical Methods in Fluids 67, 1456-1469 (2011).

[22] L. G. Leal, ed., CHAPTER 7 - Thin Films, Lubrication, and Related Problems (Butterworth-Heinemann, Boston) (1992).

[23] A. A. Korobkin, A. S. Ellis, and F. T. Smith, "Trapping of air in impact between a body and shallow water", Journal of Fluid Mechanics 611, 365-394 (2008).

[24] E. Klaseboer, J. P. Chevaillier, C. Gourdon, and O. Masbernat, "Film drainage between colliding drops at constant approach velocity: Experiments and modeling", J. Coll. Int. Sci. 229, 274-285 (2000).

[25] W. Bouwhuis, K. G. Winkels, I. R. Peters, P. Brunet, D. Van Der Meer, and J. H. Snoeijer, "Oscillating and star-shaped drops levitated by an airflow", Phys. Rev. E 88, 023017 (2013),

See Chapter 6 of this thesis.

[26] R. C. A. Van der Veen, T. Tran, D. Lohse, and C. Sun, "Direct measurements of air layer profiles under impacting droplets using high-speed color interferometry", Phys. Rev. E 85, 026315 (2012).

[27] M. H. W. Hendrix, R. Manica, E. Klaseboer, D. Y. C. Chan, and C. D. Ohl, "Spatiotemporal evolution of thin liquid films during impact of water bubbles on glass on a micrometer to nanometer scale", Phys. Rev. Lett. 108, 247803 (2012).

[28] J. R. Saylor and G. D. Bounds, "Experimental study of the role of the weber and capillary numbers on mesler entrainment", AIChE Journal 58, 3841-3851 (2012). 
[29] Q. Sun, E. Klaseboer, B. C. Khoo, and D. Y. C. Chan, "A robust and nonsingular formulation of the boundary integral method for the potential problem", Engineering Analysis with Boundary Elements 43, 117-123 (2014).

[30] R. C. A. Van der Veen, M. H. W. Hendrix, T. Tran, C. Sun, P. A. Tsai, and D. Lohse, "How microstructures affect air film dynamics prior to drop impact", Soft Matter 10, 3703-3707 (2014). 


\title{
4 \\ Initial surface deformations during impact on a liquid pool *
}

\begin{abstract}
A tiny air bubble can be entrapped at the bottom of a solid sphere that impacts onto a liquid pool. The bubble forms due to the deformation of the liquid surface by a local pressure buildup inside the surrounding gas, as also observed during the impact of a liquid drop on a solid wall. Here, we perform a perturbation analysis to quantitatively predict the initial deformations of the free surface of a liquid pool as it is approached by a solid sphere. We study the natural limits where the gas can be treated as a viscous fluid (Stokes flow) or as an inviscid fluid (potential flow). For both cases we derive the spatio-temporal evolution of the pool surface, and recover some of the recently proposed scaling laws for bubble entrapment. On inserting typical experimental values for the impact parameters, we find that the bubble volume is mainly determined by the effect of gas viscosity.
\end{abstract}

${ }^{*}$ Published as: W. Bouwhuis, M.H.W. Hendrix, D. van der Meer, J.H. Snoeijer, "Initial surface deformations during impact on a liquid pool", J. Fluid Mech. 771, 503-519 (2015). 


\subsection{Introduction}

The phenomena resulting from solid-body impacts on liquid surfaces are widely studied because of their omnipresence in nature and industry [1-8]. These involve splashing, jet formation, cavity formation, and air bubble entrapment. The entrapment of tiny micrometer-sized air bubbles between a solid object and a pool is due to a mechanism similar to that of the impact of a liquid drop on a solid surface [9-15] or of a drop onto a liquid pool [16-19]. The air that surrounds the falling object is squeezed out between the solid and the pool surface during the final stages of impact, resulting in a local pressure build-up in the gas. This pressure will induce a small deformation of the liquid surface (Fig. 4.1b), which will finally result in the entrapment of a tiny air bubble by the rupture of the enclosed air film (Fig. 4.1c). For many applications these air bubbles are undesirable, and hence, the prediction of their size is of great importance.

There are two main types of theoretical approach to determine the deformations of the liquid surface and predict the size of the entrapped air bubble, namely full numerical solutions of the problem and scaling arguments $[7,13-$ $15,17]$. Combined with experiments, these have led to the observation that the size of the air bubble for impact of a liquid drop on a flat solid surface is determined by either the inertia of the liquid or the surface tension, as described in Chapter 2 of this thesis. For increasingly high impact velocities, liquid inertia dominates and reduces the size of the air film at impact ('inertial regime'), while surface tension dominates for lower velocities or smaller drop sizes ('capillary regime'). The case of a solid sphere impacting on a pool leads to similar bubble entrapment, and, moreover, in the inertial regime the same scaling law (including the multiplicative prefactor) as for the impact of a drop on a solid has been observed [6, 19]. Here, the final center height difference between the two surfaces, which is called the dimple height $H_{d}$, and the entrapped bubble volume $V_{b}$ respectively scale as

$$
H_{d} \sim R \mathrm{St}^{-2 / 3}, \quad V_{b} \sim R^{3} \mathrm{St}^{-4 / 3} .
$$

Here, St is the Stokes number, St $=\rho_{l} U R / \eta_{g}$, in which $\rho_{l}$ is the density of the liquid, $R$ is the radius of the drop, $U$ is the impact velocity and $\eta_{g}$ is the dynamic viscosity of the air. This scaling has been confirmed experimentally and numerically $[6,7,13,14,17,19]$. On the other hand, in the capillary regime (small velocities or small drops), the scaling analysis predicts $[14,16]$ 


$$
H_{d} \sim R \mathrm{Ca}^{1 / 2}, \quad V_{b} \sim R^{3} \mathrm{Ca},
$$

where $\mathrm{Ca}=\eta_{g} U / \gamma$ is the capillary number based on the gas properties and surface tension $\gamma$. The crossover between the two regimes, at which the size of the entrapped air bubble is maximal, is found by equating the predictions for $H_{d}$ from Eqs. (4.1) and (4.2). Then, one finds $U_{0} \sim \eta_{g}^{1 / 7} \gamma^{3 / 7} /\left(\rho_{l}^{4 / 7} R^{4 / 7}\right)$, where $U_{0}$ is the crossover impact velocity, leading to maximal bubble entrapment. For an impacting water drop having a radius of $1 \mathrm{~mm}$, this gives $0.07 \mathrm{~m} / \mathrm{s}$. Indeed, this is of the same order of magnitude as was observed experimentally, where the maximum bubble size was found at approximately $0.25 \mathrm{~m} / \mathrm{s}$ (for ethanol drops) (see Chapter 2 of this thesis). Generically, for drops or spheres falling at their terminal velocity of a few meters per second, the impact thus takes place in the inertial regime, where the effect of surface tension can thus be neglected when focusing on the air bubble entrapment. Note that surface tension will enter during the rupture of the air film, which, however, appears to be on a different time scale. In Chapter 2 it was experimentally found that,
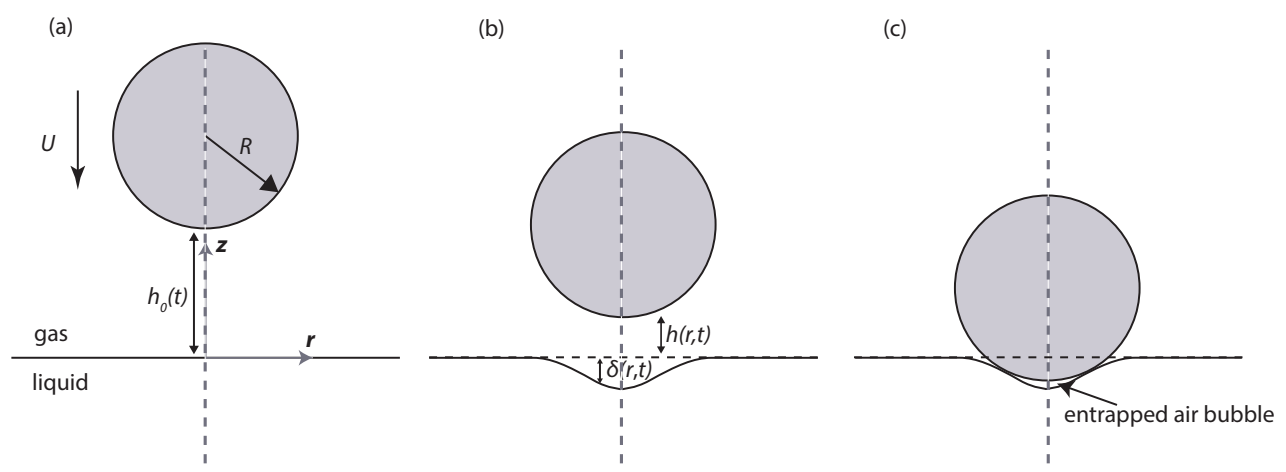

Figure 4.1: (a) A solid sphere (radius $R$ ) approaches a liquid surface with velocity $U$. The gap height between the bottom of the sphere and the undisturbed water level $(z=0)$ is $h(r, t), r$ and $t$ being the radial coordinate and time, respectively, with $h(0, t)=h_{0}(t)$. (b) While the sphere moves downwards, the pool deflects by a small amount $\delta(r, t)$, as a result of the local pressure build-up in the air that is squeezed out. In the limit where $\delta \ll h_{0}$, which typically is valid up to very close to the impact time, the profiles are computed analytically. (c) This mechanism will finally result in air bubble entrapment. 
in the inertial regime, the bubble volume was fixed before the rupture of the air film.

In this chapter, we analytically compute the initial deformations due to sphere impact onto a liquid pool in the inertial regime, where the deflection of the liquid is limited by its inertia rather than by its surface tension. In experiments, there is generally not enough resolution to accurately detect these initial deformations, and therefore we use numerical simulations, which also provide a bridge towards larger deformations. By restricting ourselves to small deformations of the pool surface, we obtain detailed spatio-temperal information on the deflection as well as the dependence on experimental parameters. This provides a natural bridge between scaling theory, which lacks detailed information on the structure of the interface deflection, and profiles obtained by direct numerical simulations. Similar calculations were previously performed by Yiantsios et al., 1990 [16] in the capillary regime, recovering the scaling (4.2). Hence, such a small-deformation theory gives an analytical foundation to the scaling laws, as well as detailed predictions of the shape of the deformation. Although the problem of a cushioning air layer has been solved by Wilson et al., 1991 [20] for an 'inertial' air layer, a similar insightful similarity analysis for the inertial (liquid) regime has not yet been attempted.

This chapter is organized as follows. Sec. 4.2 starts with a dimensional analysis of the problem and shows the limiting cases when the gas can be described as a potential flow or as a viscous lubrication flow. This section also outlines the formalism based on which the interface deformations are computed. In Sec. 4.3 we present the results for both viscous gas flow and potential gas flow. The analytical results are illustrated for a representative case of impact on a pool of water, with a sphere of radius $R=1 \mathrm{~mm}$ and velocity $U=5 \mathrm{~m} / \mathrm{s}$, surrounded by air, as is typical in experiments (inertial regime). Here we also provide a detailed comparison of our results with numerical simulations based on the Boundary Integral (BI) method, to validate our analysis and to investigate when the results start to deviate from the small-deformation regime. In Sec. 4.4 we conclude on the results in terms of air bubble entrapment.

\subsection{Formulation}

The geometry of the problem is sketched in Fig. 4.1: we consider a solid sphere (radius $R$ ) moving downwards towards a pool with a velocity $U$ (Fig. 4.1a). The velocity of the sphere during its fall is assumed to be constant, i.e. we 
neglect the acceleration of gravity and the possible deceleration due to the gas flow. The movement of the air induces an increase of the gas pressure at the bottom of the sphere, which will then deflect the pool surface by a distance $\delta(r, t)$ (Fig. 4.1b). The deformation $\delta$ is defined as positive when the pool deflects downwards. For as long as the interface deflection is small with respect to the height of the gap i.e. $|\delta| \ll h$, the problem can be solved by a perturbation analysis. In this section we first address the problem by dimensional analysis, and then provide the linearized formalism that allows computation of the spatio-temporal evolution of the deflection $\delta(r, t)$.

\subsubsection{Dimensional analysis}

Let us first consider the gas flow induced by the motion of the sphere. In the regime where the height of the gap is much larger than $R$, the sphere does not experience any influence of the pool. In this case, the Reynolds number of the gas flow is $\operatorname{Re}_{g}=\rho_{g} U R / \eta_{g}$, where $\rho_{g}$ is the density of air $\left(1.204 \mathrm{~kg} / \mathrm{m}^{3}\right)$. However, as soon as the gap height becomes small, $h_{0} / R \ll 1$, the airflow will be oriented mainly in the radial direction. As is typical for lubrication flows [21], one then has to consider a different Reynolds number which is obtained from the radial component of the Navier Stokes equation. In terms of scaling laws this gives $\rho_{g} u_{r}^{2} / L \sim \eta_{g} u_{r} / h_{0}^{2}$, where $u_{r}$ is the typical radial gas flow velocity, and $L=\sqrt{R h_{0}}$ is the length scale in the radial direction [7, 1315]. Application of mass conservation on the air gives $U L \sim u_{r} h_{0}$, and after elimination of $u_{r}$ one thus finds the relevant Reynolds number $\operatorname{Re}_{g}$, lubr. $=$ $\rho_{g} U h_{0} / \eta_{g}$. In the thin-gap regime, the relative influence of the viscosity and the inertia of the gas thus involves the gap thickness $h_{0}$ instead of the sphere radius $R$.

It is instructive to evaluate these parameters for typical experimental values, such as spheres falling in air $\left(\rho_{g}=1.204 \mathrm{~kg} / \mathrm{m}^{3}, \eta_{g}=1.82 \times 10^{-5} \mathrm{~Pa} \mathrm{~s}\right)$ with $R=1 \mathrm{~mm}$ and $U=5 \mathrm{~m} / \mathrm{s}$. The crossover from inertial to viscous gas flow, $\operatorname{Re}_{g}$, lubr. $\sim 1$, arises when $h_{0} \sim 3 \mu \mathrm{m}$. This implies that there exists an "inertial thin-gap regime", where $h_{0} / R<1$ and $\operatorname{Re}_{g}$, lubr. $>1$ at the same time. Only for the final stages of the impact, $h_{0}<3 \mu \mathrm{m}$, can the gas be described by a purely viscous flow. In the remainder of this chapter, we therefore consider a potential flow analysis during two parts of the trajectory: the large-gap stage $h_{0} / R \gg 1$, and the thin-gap stage $h_{0} / R \ll 1$. The viscous flow is treated only in the final stages of impact, for which $h_{0} / R \ll 1$ and it is thus justified to reside to lubrication theory. The various limits will be worked out separately in Sec. 4.3 . 
The liquid pool is assumed to be a low-viscosity liquid and is treated for small-amplitude deformations. These are essentially the same assumptions as for the propagation of linear surface waves [22], where the amplitude is small with respect to the length scales of the problem. We focus on the "inertial regime" of impact, where the deformation is limited by the acceleration of the liquid and not by the surface tension of the liquid-air interface. The influence of gravity will also be neglected in the theory; the Froude number based on the impact parameters, $\mathrm{Fr}=U^{2} /(g R)$, is much larger than 1 .

\subsubsection{From gas pressure to interface deflection}

The first step of the analysis is to compute the response of the liquid on a gas pressure $P_{g}$ for the different limiting cases (viscous/inertial gas), as discussed above. Since we set out to compute the initial deformation, we can compute $P_{g}$ assuming that the liquid pool is undeformed - the influence of a finite deflection is a correction at higher order in $\delta / h$. We assume axisymmetry and solve the equations in cylindrical coordinates $(r, z)$ (see Fig. 4.1). The gas pressure will provide the boundary condition at the liquid pool, generating a liquid flow as described by the linearized Euler equation:

$$
\frac{\partial \vec{v}}{\partial t}=-\frac{1}{\rho_{l}} \vec{\nabla} P_{l}
$$

where $\vec{v}(r, z, t)$ is the velocity field in the liquid and $P_{l}(r, z, t)$ is the pressure inside the liquid. The advection terms in the Euler equation are quadratic in velocity and therefore of higher order in $\delta / h$, in analogy to the wave analysis [22]. Without surface tension, the gas pressure provides the boundary condition for the liquid pressure,

$$
P_{l}(r, z=-\delta, t) \simeq P_{l}(r, z=0, t)=P_{g}(r, t),
$$

with the first equality again due to taking into account only leading order terms in $\delta / h$. The resulting deflection is given by the kinematic boundary condition:

$$
\frac{\partial \delta}{\partial t}=-\left.v_{z}\right|_{z=-\delta}-\left.v_{r}\right|_{z=-\delta} \frac{\partial \delta}{\partial r} \simeq-\left.v_{z}\right|_{z=0},
$$

where $\left.v_{z}\right|_{z=0}$ is the vertical velocity at the pool surface (to the lowest order in $\delta / h)$. Substitution of condition (4.5) into the vertical component of Eq. (4.3) gives 


$$
\frac{\partial^{2} \delta}{\partial t^{2}}=\left.\frac{1}{\rho_{l}} \frac{\partial P_{l}}{\partial z}\right|_{z=0}
$$

The above equation shows that in order to compute $\delta(r, t)$, one requires a spatial derivative $\partial P_{l} / \partial z$. Hence, we need to find the pressure distribution inside the liquid that is induced by $P_{g}$ at the free surface. For an incompressible liquid this can be achieved by taking the divergence of Eq. (4.3), which due to $\vec{\nabla} \cdot \vec{v}=0$ reduces to $\nabla^{2} P_{l}=0$. As the boundary condition is axisymmetric, it is natural to express the pressure as the axisymmetric solution of the Laplace equation:

$$
P_{l}(r, z, t)=\int_{0}^{\infty} \widehat{P_{g}}(k, t) \mathrm{J}_{0}(k r) \mathrm{e}^{k z} k \mathrm{~d} k
$$

where the integration variable $k$ is the wave number, and $\mathrm{J}_{0}(k r)$ is the Bessel function of the first kind with order $\nu=0$. The amplitude of the 'modes' $\mathrm{J}_{0}(k r) \mathrm{e}^{k z}$ is given by the Hankel transform of order 0 of the gas pressure $P_{g}(r, t)$,

$$
\widehat{P_{g}}(k, t)=\int_{0}^{\infty} P_{g}(r, t) \mathrm{J}_{0}(k r) r \mathrm{~d} r .
$$

Substitution of this expression for the pressure into Eq. (4.6) gives

$$
\frac{\partial^{2} \delta}{\partial t^{2}}(r, t)=\int_{0}^{\infty} \frac{\widehat{P_{g}}(k, t)}{\rho_{l}} \mathrm{~J}_{0}(k r) k^{2} \mathrm{~d} k
$$

where we note an additional factor $k$ coming from the derivative of $\partial P_{l} / \partial z$.

The basic procedure for determining $\partial^{2} \delta / \partial t^{2}$ from the gas pressure is now clear: one needs to find the Hankel transform of the gas pressure (Eq. (4.8)), subsequently take the derivative of the result in the $z$-direction and evaluate the expression at $z=0$, and finally take the inverse Hankel transform (Eq. (4.9)). In the following section we will perform these steps for the gas pressure computed in the limits of Stokes gas flow and inviscid gas flow. 


\subsection{Results}

\subsubsection{Stokes gas flow}

We now turn to the Stokes flow in the lubrication limit, which is valid for $\operatorname{Re}_{g}$, lubr. $\ll 1$ and $h_{0} / R \ll 1$. In the case of vanishing interface deformation, the gas pressure building up below an impacting sphere becomes $[16,23]$

$$
P_{g}(r, t)=\frac{3 \eta_{g} U R}{h_{0}^{2}\left(1+\frac{r^{2}}{2 R h_{0}}\right)^{2}}=\frac{3 \eta_{g} U}{R}\left(\frac{R}{L}\right)^{4} F_{1}(u) .
$$

Here, we factorized the result in dimensional parameters determining the magnitude of the pressure and a dimensionless function $F_{1}(u)$ that contains the spatial information on the pressure profile. For this, we introduced $L(t)=$ $\sqrt{R h_{0}(t)}$ as the relevant radial length scale, while the geometrical function reads

$$
F_{1}(u)=\frac{1}{\left(1+\frac{1}{2} u^{2}\right)^{2}} ; \quad u(t)=\frac{r}{L(t)} .
$$

Note that in the limit of vanishing thickness $h_{0}$, the pressure tends to diverge, $P_{g} \sim h_{0}^{-2}$, while the width of the peak becomes increasingly small, $L \sim h_{0}^{1 / 2}$. These singular tendencies are regularized when the deformations of the surface become comparable to $h_{0}$, but still set the characteristic scales for the enclosed bubble volume.

We continue the analysis by inserting the gas pressure profile in Eq. (4.9), and find a closed form expression:

$$
\frac{\partial^{2} \delta}{\partial t^{2}}(r, t)=\frac{3 \eta_{g} U}{\rho_{l} R L}\left(\frac{R}{L}\right)^{4} G_{1}(u)
$$

Once more we recognize a dimensional prefactor that determines the scale of the acceleration, while the time dependence follows from $L(t)$ and $u(t)$, and the spatial dependence through $G_{1}(u)$. The additional factor $1 / L$ appearing in (4.12) originates from the scaling $u=r / L$. The spatial similarity profile is $G_{1}(u)=\int_{0}^{\infty} \widehat{F_{1}} \mathrm{~J}_{0}(k u) k^{2} \mathrm{~d} k$, where $\widehat{F_{1}}(k)$ is the Hankel transform of $F_{1}(u)$. The analytical expression for $\widehat{F_{1}}(k)$ is found to be

$$
\widehat{F_{1}}(k)=\sqrt{2} k \mathrm{~K}_{1}(\sqrt{2} k),
$$


where $\mathrm{K}_{1}(k)$ is the modified Bessel function of the second kind with order $\nu=1$, and the analytical expression for $G_{1}(u)$ is

$$
G_{1}(u)=\frac{-8 \mathrm{~K}\left(\frac{u}{\sqrt{u^{2}+2}}\right)-\mathrm{E}\left(\frac{u}{\sqrt{u^{2}+2}}\right)+14 \mathrm{E}\left(\frac{u}{\sqrt{u^{2}+2}}\right)}{\left(u^{2}+2\right)^{5 / 2}} .
$$

$\mathrm{K}$ and $\mathrm{E}$ are the complete elliptic integrals of the first and second kind respectively.

To illustrate and validate our analysis, we compare the predicted profiles with the results obtained by Boundary Integral (BI) simulations [24-26]. The simulation method is the same as in Chapter 2 of this thesis: the liquid within the pool is described as a potential flow, while the pressure along the pool surface is explicitly calculated from the viscous lubrication equation for the gas flow. To be able to confirm our theoretical predictions in the inertial regime without the influences of surface tension and hydrostatics (which are both very small, as mentioned in the Introduction), $\gamma$ and $g$ are equal to zero in our simulations. In the limit of small deflection, the simulations should thus recover Eq. (4.12).

Figure $4.2 \mathrm{a}$ shows the configuration on the scale of the sphere, for typical impact parameters for a sphere in air $(R=1 \mathrm{~mm}, U=5 \mathrm{~m} / \mathrm{s})$. The interface deflection $\delta$ is shown in Fig. 4.2b, at the moment when the sphere is at a height $h_{0}=100 \mu \mathrm{m}$. At this time, $\delta \ll h_{0} \ll R$, for which we expect agreement between the BI results and our prediction from Eq. (4.12). Figure 4.2c shows the acceleration $\partial^{2} \delta / \partial t^{2}$ versus $r$. The solid line is the result from the BI simulations and indeed gives perfect agreement with the prediction, represented by the dots.

The actual deflection profile $\delta(r, t)$ can not be integrated explicitly from (4.12), due to the time dependence through $L$ and $u$. However, we can derive $\left.\delta\right|_{r=0}$, the deflection of the pool surface on the axis, which does not involve $L(t)$. Using that $\partial / \partial t=-U \partial / \partial h_{0}$, we find

$$
\frac{\left.\partial^{2} \delta\right|_{r=0}}{\partial h_{0}^{2}}=\frac{3 \eta_{g} G_{1}(0)}{\rho_{l} U R^{2}}\left(\frac{R}{L}\right)^{5}=\frac{3 \eta_{g} G_{1}(0)}{\rho_{l} U R^{2}}\left(\frac{R}{h_{0}}\right)^{5 / 2},
$$

where (4.14) implies $G_{1}(0)=\frac{3}{8} \sqrt{2} \pi$. The solution of Eq. (4.15) for $\left.\delta\right|_{r=0}$ is subject to start-up effects as long as $h_{0} \sim h_{s}$, where $h_{s}$ is the initial height of the gap. If we let the initial height $h_{s} \rightarrow \infty$, we find

$$
\left.\delta\right|_{r=0} \simeq \frac{3}{2} \sqrt{2} \pi \frac{\eta_{g}}{\rho_{l} U}\left(\frac{R}{h_{0}}\right)^{1 / 2}
$$



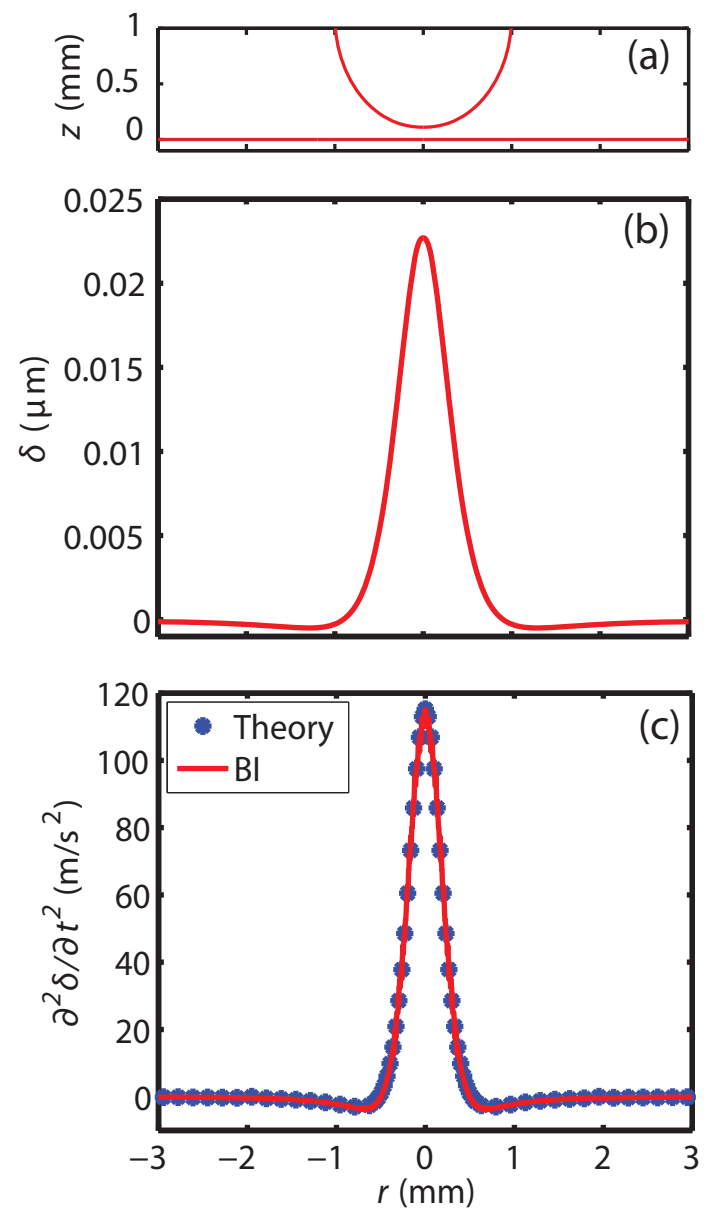

Figure 4.2: Deflection of the pool interface for Stokes gas flow; $R=1 \mathrm{~mm}$, $U=5 \mathrm{~m} / \mathrm{s}$, starting height of the (bottom of the) sphere $h_{s}=0.5 \mathrm{~mm}$, current height: $h_{0}=0.1 \mathrm{~mm}$. (a) Global view of the sphere and pool contours, (b) the pool deflection $\delta$ as a function of $r$, and (c) $\partial^{2} \delta / \partial t^{2}$ as a function of $r$. The solid red lines result from the Boundary Integral (BI) simulation. The theoretical result from Eq. (4.12) has been superimposed in panel c (blue dots). Note the difference in scales on the vertical axes of panel a and b. The BI results agree perfectly with the theoretical predictions, as long as $|\delta| \ll h$. 


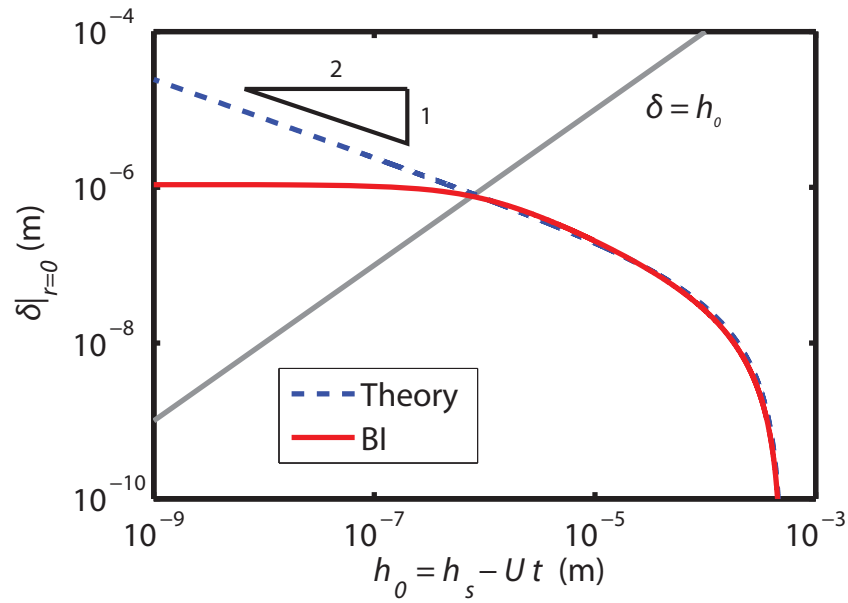

Figure 4.3: Deflection of the pool interface on the axis, $\delta_{r=0}$, plotted against $h_{0}(t)$, for Stokes gas flow; $R=1 \mathrm{~mm}, U=5 \mathrm{~m} / \mathrm{s}$, starting height $h_{s}=0.5 \mathrm{~mm}$. The solid red line is the result from the BI simulation. The theoretical result from Eq. (4.15) has been superimposed. After a start-up regime for large $h_{0}$, the deflection $\left.\delta\right|_{r=0}$ converges towards a $-1 / 2$ power-law. The BI results perfectly agree with the theoretical predictions, until $\delta$ and $h_{0}$ become of comparable magnitude, pointed out by the crossing with the solid gray line $\left.\delta\right|_{r=0}=h_{0}$. At that moment $\delta_{r=0}$ saturates to a constant value, which is the 'dimple height' $H_{d}$ of Chapter 2 of this thesis. 
This predicts that the central height increases dramatically when $h_{0}$ decreases, as $\delta \sim h_{0}^{-1 / 2}$. Figure 4.3 shows the BI result for $\left.\delta\right|_{r=0}$ against $h_{0}$ (solid line), superimposed with the theoretical predictions (dashed line, taking into account the finite initial height $\left.h_{s}\right)$. Indeed, as soon as $h_{0} \ll h_{s},\left.\delta\right|_{r=0}$ converges to a $-1 / 2$ power law. As expected, the simulation results depart from the analytical prediction when $\delta \sim h_{0}$ (indicated by the solid gray line) and the lubrication approximation ceases to be valid. At this point, the deflection converges to a constant, which will be the final dimple height $H_{d}$. As stated in the Introduction, this will determine the dimple volume, and thus the entrapped air bubble volume, independently of the air film rupture process.

The current analysis provides a rigorous foundation for the scaling results obtained previously in Refs. $[6,7,13,14,17]$. There, the 'dimple height' $H_{d}$ was observed to approach a constant value during the final stages of the impact. Figure 4.3 shows that this height can be estimated from $\delta_{r=0} \sim h_{0} \sim H_{d}$. Using (4.16), this immediately gives

$$
H_{d} \sim \frac{\eta_{g} R^{1 / 2}}{\rho_{l} U H_{d}^{1 / 2}} \sim R \mathrm{St}^{-2 / 3},
$$

where St $=\rho_{l} U R / \eta_{g}$ is the Stokes number. The corresponding volume of the entrapped bubble volume then scales as

$$
V_{b} \sim L^{2} H_{d} \sim R H_{d}^{2} \sim R^{3} \mathrm{St}^{-4 / 3}
$$

where we use the common estimate that $L$ sets the lateral scale of the bubble. These are precisely the scaling predictions for the inertial regime (for Stokes gas flow) where the assumptions $H_{d} \sim \delta$ and $L \sim\left(H_{d} R\right)^{1 / 2}$ were further validated $[6,7,13,14,17,19]$.

\subsubsection{Potential gas flow}

As motivated in Sec. 4.2.1, the inertial phase of the impacting sphere consists of two distinct stages: the large-gap regime $h_{0} \gg R$ and the thin-gap regime $h_{0} \ll R$. Below we separately treat both limiting cases analytically. We furthermore perform a numerical potential flow calculation for the full range of $h_{0} / R$, to validate the analysis and to show how the two stages are connected. 
Large-gap regime: $h_{0} \gg R$

When the sphere is very far from the pool surface, the flow field can be described by the well-known potential flow field around a moving sphere of radius $R$. The introduction of the (undeformed) pool surface, however, requires that the gas velocity has no vertical component, or $\left.v_{z}\right|_{z=0}=0$. This boundary condition can be satisfied using the 'method of images', corresponding to two approaching spheres having radius $R$ with approaching velocity $U$ towards a mirroring horizontal line $(z=0)$. By applying the superposition of the potentials for the two moving spheres, one obtains the potential

$$
\phi(r, z, t)=\frac{U R^{3}}{2}\left[\frac{\left(z-R-h_{0}\right)}{\left(r^{2}+\left(z-R-h_{0}\right)^{2}\right)^{3 / 2}}-\frac{z+R+h_{0}}{\left(r^{2}+\left(z+R+h_{0}\right)^{2}\right)^{3 / 2}}\right] .
$$

It is important to realize that the introduction of the second moving sphere not only influences the flow around $z=0$, but also gives a small unwanted velocity on the boundary of the original sphere. In the limit of very large gaps, $R / h_{0} \ll 1$, this correction becomes negligible and (4.19) gives the asymptotically correct potential.

We now extract the gas pressure profile on the level of the pool surface $z=0$, by applying the unsteady Bernoulli equation:

$$
P_{g}(r, t)=\rho_{g} U^{2}\left[\left(\frac{R}{\zeta}\right)^{3} F_{2}(u)+\frac{9}{2}\left(\frac{R}{\zeta}\right)^{6} F_{3}(u)\right] \simeq \rho_{g} U^{2}\left(\frac{R}{\zeta}\right)^{3} F_{2}(u)
$$

Here, $\zeta(t)=R+h_{0}(t)=R+h_{s}-U t$, the radial direction is scaled as $u(t)=r / \zeta$, while the spatial profiles are

$$
\begin{aligned}
& F_{2}(u)=\frac{2-u^{2}}{\left(1+u^{2}\right)^{5 / 2}} \\
& F_{3}(u)=\frac{-u^{2}}{\left(1+u^{2}\right)^{5}} .
\end{aligned}
$$

Since $(4.19,4.20)$ are only valid for $h_{0} \gg R$, we only keep the dominant first term in (4.20). Note that the width of the pressure peak is now set by the scale $\zeta=h_{0}+R$. This can be contrasted with the width in the thin-gap limit, $L=\sqrt{R h_{0}}$, which becomes very narrow. 
Next, from (4.20) we can compute the induced acceleration profile using (4.9):

$$
\frac{\partial^{2} \delta}{\partial t^{2}}(r, t)=\frac{\rho_{g} U^{2}}{\rho_{l} \zeta}\left(\frac{R}{\zeta}\right)^{3} G_{2}(u)
$$

One recognizes a dimensional prefactor that is separated from the spatiotemporal dependence. The function $G_{2}(u)=\int_{0}^{\infty} \widehat{F_{2}} \mathrm{~J}_{0}(k u) k^{2} \mathrm{~d} k$ is the spatial similarity profile, where $\widehat{F_{2}}(k)$ is the Hankel-transform of $F_{2}(u)$. For $G_{2}(u)$ we did not find any analytical expression, but one can numerically calculate the given integral (cf. Fig. 4.4).

Once again, we can analytically compute the behavior of the central deflection, $\left.\delta\right|_{r=0}$ :

$$
\frac{\left.\partial^{2} \delta\right|_{r=0}}{\partial h_{0}^{2}}=\frac{\rho_{g} G_{2}(0)}{\rho_{l} R}\left(\frac{R}{\zeta}\right)^{4} .
$$

Recalling that $\partial / \partial h_{0}=\partial / \partial \zeta$ and $\zeta \rightarrow 2 R$ for $h_{0} \rightarrow R$, this implies that the final $\delta_{r=0}$ scales as $\rho_{g} R / \rho_{l}$. In contrast to the result for viscous flow, the typical deformation depends only on the density ratio $\rho_{g} / \rho_{l}$, and not on the impact velocity. While the density ratio is typically small, we anticipate that the resulting deflection for a millimeter-sized sphere can be a few microns. This is actually comparable to typical deflections in the viscous lubrication phase. However, the pool is not deformed locally over a small width $\sqrt{R h_{0}}$, but over the scale of the entire sphere, and therefore it will be of little consequence for the formation of the dimple and the size of the entrapped air bubble.

Thin-gap regime: $h_{0} \ll R$

In the inertial thin-gap limit, the gas is squeezed out mainly in the radial direction. To predict the pressure profile for this stage of the impact, we use the depth-integrated continuity equation $[27,28]$

$$
\frac{\partial h}{\partial t}+\frac{1}{r} \frac{\partial}{\partial r}\left(r h \bar{u}_{r}\right)=0
$$

where $\bar{u}_{r}(r, t)$ is the height-averaged radial gas velocity in the gap. Assuming a plug flow that does not depend on the $z$-coordinate, this average simply gives $\bar{u}_{r}(r, t)=u_{r}(r, t)$. This analytical description is similar to that of Wilson et al., 1991 [20], where cushioning air-layers at solid-liquid impact in the inertial regime are also studied, although in 2D Cartesian coordinates, for 
general shapes of the impacting solid. In the present case, the bottom of the impacting solid sphere can be described as $h=h_{0}(t)+r^{2} /(2 R)$, and thus $\partial h / \partial t=\partial h_{0} / \partial t=-U$. Hence, we can integrate (4.25) to find

$$
u_{r}=\bar{u}_{r}=\frac{U r}{2 h_{0}\left(1+\frac{r^{2}}{2 R h_{0}}\right)} .
$$

The velocity profile (4.26) has a local maximum at $r=\sqrt{2 R h_{0}}$, and vanishes for $r=0$ and $r=\infty$. Substitution of the profile into the radial component of the Euler equation and integration over $r$ gives the gas pressure:

$$
P_{g}(r, t)=\frac{\rho_{g} U^{2} R}{2 h_{0}}\left(\frac{1+\frac{r^{2}}{4 R h_{0}}}{\left(1+\frac{r^{2}}{2 R h_{0}}\right)^{2}}\right)=\frac{\rho_{g} U^{2}}{2}\left(\frac{R}{L}\right)^{2} F_{4}(u),
$$

with $L(t)=\sqrt{R h_{0}(t)}, u(t)=r / L$, and

$$
F_{4}(u)=\frac{1+\frac{1}{4} u^{2}}{\left(1+\frac{1}{2} u^{2}\right)^{2}}
$$

Note that the geometry of the thin-gap again gives rise to a highly localized pressure profile of width $\sqrt{R h_{0}}$. The gas pressure again tends to diverge as $h_{0} \rightarrow 0$, but more slowly than in the viscous case: the inertial gas pressure in the thin-gap-limit is proportional to $1 / h_{0}$, in contrast to the more singular scaling for the viscous gas flow scenario, $1 / h_{0}^{2}$.

From (4.9) we deduce the pool surface acceleration

$$
\frac{\partial^{2} \delta}{\partial t^{2}}(r, t)=\frac{\rho_{g} U^{2}}{2 \rho_{l} L}\left(\frac{R}{L}\right)^{2} G_{4}(u)
$$

where $G_{4}(u)=\int_{0}^{\infty} \widehat{F_{4}} \mathrm{~J}_{0}(k u) k^{2} \mathrm{~d} k$, with $\widehat{F_{4}}(k)$ the Hankel-transform of $F_{4}(u)$. At the origin $r=0$, this reduces to

$$
\frac{\left.\partial^{2} \delta\right|_{r=0}}{\partial h_{0}^{2}}=\frac{\rho_{g} G_{4}(0)}{2 \rho_{l} R}\left(\frac{R}{L}\right)^{3} .
$$

Just like in the case of the large-gap regime, the central deflection has no dependence on the impact velocity. Solving gives $\delta_{r=0} \sim h_{0}^{1 / 2}+$ integration constants. From this we conclude that in the inertial thin-gap limit, the pressure tends to diverge for $h_{0} \rightarrow 0$, but the deflection $\delta$ converges. Contrarily 
to the final stages in the case of viscous gas flow, the inertial gas pressure is not sufficiently singular to induce a strongly enhanced deflection. The integration constants depend on the full history of the impact process, which thus involves the dynamics during the preceding large-gap regime. To predict the actual deflection during the final stages of sphere impact, it is thus not sufficient to consider the large-gap or thin-gap regime of the potential gas flow problem: numerical simulation of the full impact process over all $h_{0} / R$ is required.

\section{Numerical simulations}

Simulation of the potential gas flow impact process using the BI technique calls for a different approach with respect to the case of Stokes gas flow. The reason is that we require the gas pressure over the full range of gap thickness, including $h_{0} \sim R$, for which no analytical solution for the gas pressure is available that can serve as a boundary condition for the liquid pool. As a consequence, the gas phase must also be computed numerically, which we achieve using the BI code. We thus need to run two separate simulations. The process is started by a BI simulation of a solid sphere impacting towards an undeformed surface, with a potential gas flow in between. From this simulation, the gas pressure profile along the pool surface $(z=0)$ is extracted. In the second BI simulation, this pressure is applied on a deformable pool surface, from which we eventually determine the resulting pool deflections. This is again a valid method as long as $\delta / h \ll 1$. The pressure data are transmitted from the first simulation to the second simulation through an extensive data file. Note that in performing two separate simulations, one needs to take into account the different length scales during the impact process (for $h_{0}=10 \mathrm{~mm} \rightarrow 100 \mathrm{~nm}$ ), implying very sensitive local node spacings and time dependencies. This was achieved by adapting the node spacing and time steps to ensure convergence of the numerical results.

Figures $4.4 \mathrm{a}$ and $\mathrm{b}$ show the configuration on the length-scale of the sphere and the interface deflection $\delta(r, t)$ respectively, for $R=1 \mathrm{~mm}, U=5 \mathrm{~m} / \mathrm{s}$ and $h_{0}=h_{s}=10 \mathrm{~mm}$ (i.e., the large-gap regime). Figure $4.4 \mathrm{c}$ shows the acceleration profile at the corresponding time, and it is observed to agree very well with the asymptotic result of Eq. (4.23) (blue dots). The very small difference between the BI result and the theoretical predictions can be explained by the fact that $h_{s} / R=10$, implying an expected difference of approximately $10 \%$ between the theory and the numerical simulations. We remark that the corresponding deformation (Fig. 4.4b) is very small, as we look at the very initial 

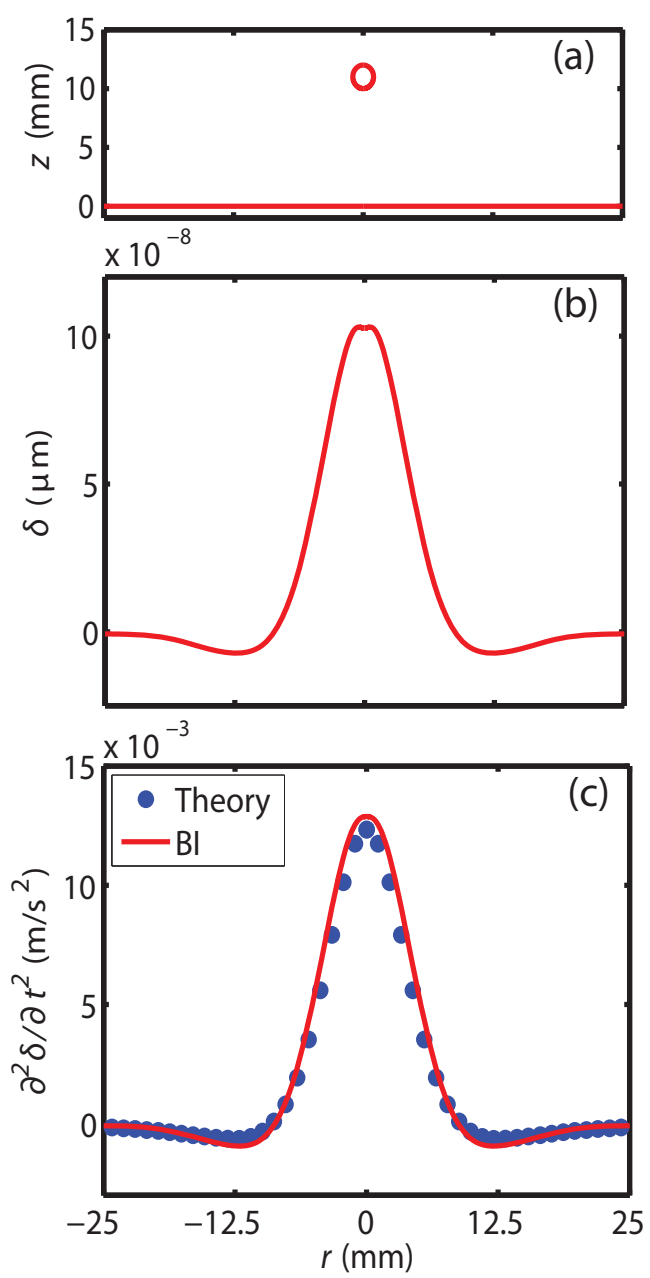

Figure 4.4: Deflection of the pool interface for potential gas flow in the limit $h_{0} \gg R ; R=1 \mathrm{~mm}, U=5 \mathrm{~m} / \mathrm{s}, h_{0}=h_{s}=10 \mathrm{~mm}$ (thus, $h_{0} / R=10$ ). (a) Global view plot of the sphere and pool contours, (b) $\delta$ against $r$, and (c) $\partial^{2} \delta / \partial t^{2}$ against $r$. The solid red lines result from the BI simulation. The theoretical result from Eq. (4.23) has been superimposed in panel c (blue dots). Note the difference in scales on the vertical axes of panel $a$ and $b$. The BI results are nicely agreeing with the theoretical predictions, until $h_{0} / R$ becomes of order 1. 

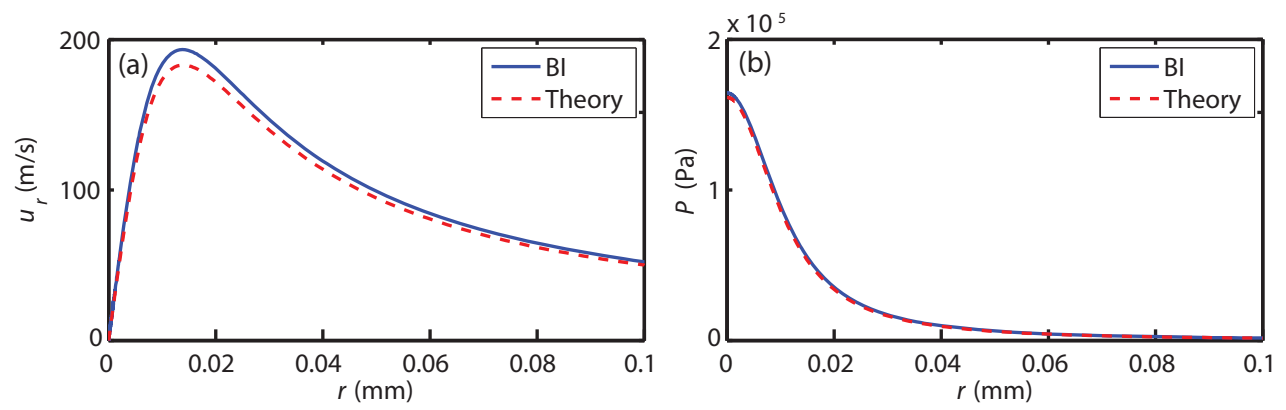

Figure 4.5: Inertial gas flow in the thin-gap limit. Theoretical prediction (red dashed line) and BI gas flow simulation result (blue solid line) of the (a) velocity and (b) pressure profile within the gas; $R=1 \mathrm{~mm}, U=5 \mathrm{~m} / \mathrm{s}$, $h_{s}=h_{0}=100 \mathrm{~nm}$ (thus, $h_{0} / R=10^{-4}$ ). We find very good agreement between the theoretical predictions and the BI results.

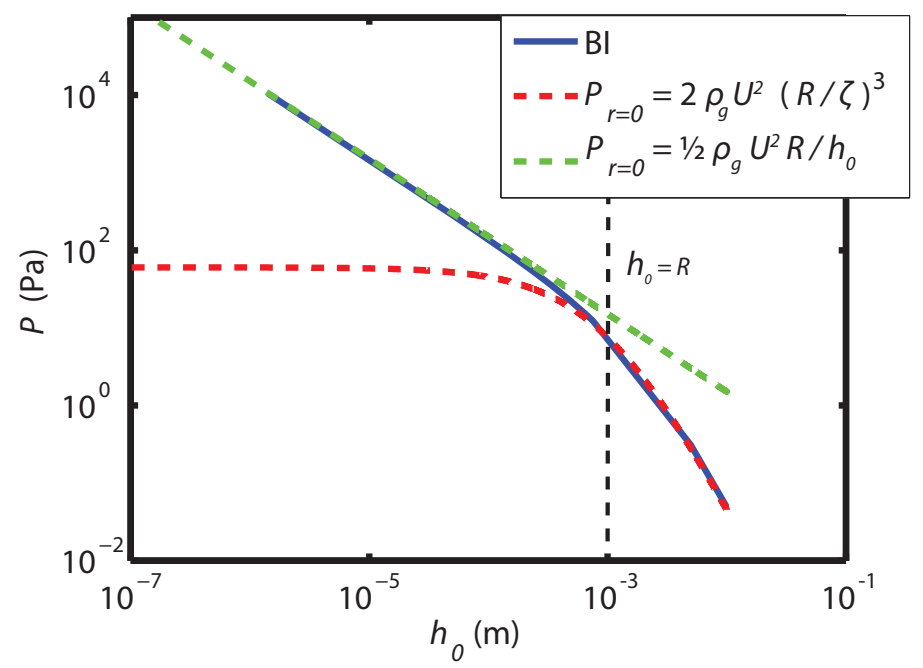

Figure 4.6: Behavior of the gas pressure on the axis, $P_{r=0}$, plotted against the gap height $h_{0}(t)$, for potential gas flow. $R=1 \mathrm{~mm}, U=5 \mathrm{~m} / \mathrm{s}, h_{s}=10 \mathrm{~mm}$. The red dashed line is the theoretical prediction in the regime $h_{0} \gg R$; the green dashed line is the theoretical prediction in the regime $h_{0} \ll R$. The dashed line points out the crossover $h_{0}=R$. The BI gas flow simulation result (blue solid line) indeed follows these predicted behaviors in the corresponding regimes, with a crossover at $h_{0} \sim R$. 


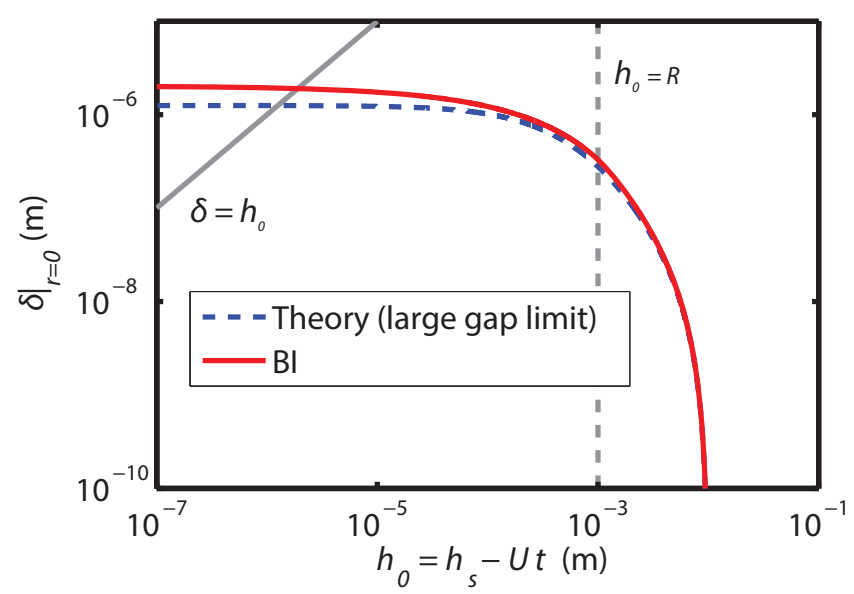

Figure 4.7: Deflection of the pool interface on the axis, $\delta_{r=0}$, plotted against the gap height $h_{0}(t)$, for potential gas flow; $R=1 \mathrm{~mm}, U=5 \mathrm{~m} / \mathrm{s}, h_{s}=$ $10 \mathrm{~mm}$. The solid red line is the result from the BI solid sphere on liquid pool simulations (Sec. 4.3.2). The theoretical result from Eq. (4.24) for the large-gap regime has been superimposed (blue dashed line). $\left.\delta\right|_{r=0}$ saturates to a constant. The BI results perfectly agree with the large-gap predictions in the regime $h_{0} \gg R$. In the regime $h_{0} \ll R, \delta_{r=0}$ deviates from this prediction, but the difference is relatively small. The dashed gray line points out the crossover $h_{0}=R$; the solid gray line points out $\left.\delta\right|_{r=0}=h_{0}$.

deformations in the start-up regime. The requirement $h_{0} / R \gg 1$ implies a large initial gap height, which, for the parameter values chosen in Fig. 4.4 to validate the asymptotics, correspond to nonphysically small deflections. The sensitiveness of the very small pool deflection gave rise to switch-off the smoothing procedure normally used within the simulations [25], such that a tiny instability remained visible around the axis, $r=0$. We confirmed that this instability has a numerical origin and that it does not influence the result on the scale of the deformations. The thin-gap regime is analyzed in Fig. 4.5. We again find very good agreement between the analytical gas velocity profile (panel a) and the pressure profile (panel b) and the BI results (here, $h_{0}=100 \mathrm{~nm}$ ).

The crossover between the large-gap and thin-gap limits is illustrated in Fig. 4.6, showing the gas pressure on the symmetry axis $r=0$. As predicted, in the limit $h_{0} / R \gg 1$ the pressure calculated by BI (blue line) equals 
$2 \rho_{g} U^{2}(R / \zeta)^{3}$ (red dashed line), and in the limit $h_{0} / R \ll 1$ the pressure equals $\rho_{g} U^{2} R /\left(2 h_{0}\right)$ (green dashed line). This confirms the validity of the analytical approaches. Finally, we investigate the deflection of the pool that is induced by the numerically obtained gas pressure. Figure 4.7 shows the deflection at $r=0$, the inertial (gas) counterpart of Fig. 4.3. As expected, $\delta_{r=0}$ deviates from the large-gap prediction in the small-gap regime, though the deviation is not very large. This means that, despite the fact that the gas pressure tends to diverge for $h_{0} \rightarrow 0$, the influence of the inertial thin-gap limit remains relatively small. For this particular example, it enhances the deflection by less than a factor of 2 . This is also one of the reasons why we do not show the corresponding theoretical profile for $\partial^{2} \delta / \partial t^{2}$, which in principle could again be directly calculated from the pressure profile. A second reason is that, in the numerical simulations, the very small gap height of $100 \mathrm{~nm}$ needs a very high local node density on both the pool surface and the sphere surface; the difference in length scales of $R$ and $h_{0}$ is four decades, which is very challenging. This necessitates very small time steps to be able to calculate a fair second derivative of the deflection profile in time. In addition, the pressure along the pool surface needs to be extracted from a prior solid-sphere-on-solid-surface simulation (through an extensive data file), which makes the discretization more complicated.

The large-gap prediction for the final $\delta_{r=0}$ is thus satisfactory, and we conclude with the following scaling law for the resulting dimple height $H_{d}$ for the inertial gas scenario as was concluded from Eq. (4.24):

$$
H_{d} \sim R \frac{\rho_{g}}{\rho_{l}} .
$$

This dimple height is independent of the impact velocity of the sphere. Since the surface deformation is the sum of the deformations in both the large-gap and the thin-gap limit, it is unclear what the correct radial and axial length scales are that lead to the volume of the pinched bubble.

\subsection{Conclusion}

We performed a perturbation analysis to investigate the initial deflections of a liquid surface, induced by the approach of an impacting solid sphere. The analysis assumed that the deflection is limited by the inertia of the liquid pool (i.e., not by its surface tension), and we considered two natural limits for the surrounding medium: Stokes gas flow and potential gas flow. We obtained 
a quantitative prediction for the pool surface deflection, which was validated numerically, and recovered previously proposed scaling laws for bubble entrapment.

While the 'cushioning' of an inertial gas layer had been analyzed before [20], most recent work on liquid or solid impact assumes a viscous gas layer. Surprisingly, our analysis reveals that inertial and viscous cushioning both lead to a pool deflection of the order of $1 \mu \mathrm{m}$, for typical experimental conditions. However, the Stokes gas pressure tends to diverge strongly for $h_{0} \rightarrow 0$, much more strongly than during the inertial gas phase. In addition, this viscous lubrication pressure profile is very localized, while most of the inertial deflection is generated during the initial phase where the pool deflection is spread over the entire width of the sphere. This explains why the experimental results on bubble entrapment are in close agreement with the scaling law (4.18) [19], while in addition (4.17) was validated for the case of a liquid drop impact on a solid $[6,7,13,14,17,19]$ : all these results are based on the viscous lubrication regime.

For completeness, we will summarize the possible scenarios for impact of a sphere onto a pool, which can be achieved for different experimental parameters. Assuming an initially high Reynolds number based on the size of the impacting object $R$, the dynamics will exhibit two different types of crossover: a geometric crossover based on the relative thickness of the gap, $h / R$, and a crossover from inertial to viscous gas flow. The order in which these crossovers occur depends on the parameters of the problem. In our numerical examples we assumed that one first reaches the thin-gap regime, before the lubrication Reynolds number (based on the gap thickness $h$ ) becomes smaller than unity. This order can be reversed for impact at smaller velocities or for a sphere sinking in a more viscous medium. In that case, however, one needs to bear in mind that the influence of the pool surface tension will become more important, corresponding to the capillary impact regime. In this case, the thin film potentially has time to drain out before a bubble is formed, making the entrapment process more complex [29, 30].

In this work, we have elaborated on the impact of a solid sphere on a liquid surface. Similar perturbation analysis can be performed for drop impact on a solid, or drop impact on a pool, although the details will be different (see Chapter 3 of this thesis). This explains why the same scaling laws are observed in all these cases. 


\section{References}

[1] A. A. Korobkin and V. V. Pukhnachov, "Initial stage of water impact", Ann. Rev. Fluid Mech. 20, 159-185 (1988).

[2] S. D. Howison, J. R. Ockendon, and S. K. Wilson, "Incompressible waterentry problems at small deadrise angles", J. Fluid Mech. 222, 215-230 (1991).

[3] A. A. Korobkin, A. S. Ellis, and F. T. Smith, "Trapping of air in impact between a body and shallow water", J. Fluid Mech. 611, 365-394 (2008).

[4] M. Do-Quang and G. Amberg, "The splash of a solid sphere impacting on a liquid surface: numerical simulation of the influence of wetting", Phys. Fluids 21(2), 022102 (2009).

[5] Q. Deng, A. V. Anilkumar, and T. G. Wang, "The phenomenon of bubble entrapment during capsule formation", J. Coll. and Interf. Sc. 333(2), $523-532$ (2009).

[6] J. O. Marston, I. U. Vakarelski, and S. T. Thoroddsen, "Bubble entrapment during sphere impact onto quiescent liquid surfaces", J. Fluid Mech. 680, 660-670 (2011).

[7] P. D. Hicks, E. V. Ermanyuk, N. V. Gavrilov, and R. Purvis, "Air trapping at impact of a rigid sphere onto a liquid", J. Fluid Mech. 695, $310-320$ (2012).

[8] M. R. Moore and J. M. Oliver, "On air cushioning in axisymmetric impacts", IMA J. Appl. Math. 79, 661-680 (2014).

[9] F. T. Smith, L. Li, and G. X. Wu, "Air cushioning with a lubrication/inviscid balance", J. Fluid Mech. 482, 291-318 (2003).

[10] D. B. van Dam and C. Le Clerc, "Experimental study of an ink-jet printed droplet on a solid substrate", Phys. Fluids 16, 3403-3414 (2004).

[11] S. T. Thoroddsen, T. G. Etoh, K. Takehara, N. Ootsuka, and A. Hatsuki, "The air bubble entrapped under a drop impacting on a solid surface", J. Fluid Mech. 545, 203-212 (2005).

[12] M. M. Driscoll and S. R. Nagel, "Ultrafast interference imaging of air in splashing dynamics", Phys. Rev. Lett. 107, 154502 (2011). 
[13] S. Mandre and M. P. Brenner, "The mechanism of a splash on a dry solid surface", J. Fluid Mech. 690, 148-172 (2012).

[14] W. Bouwhuis, R. C. A. van der Veen, T. Tran, D. L. Keij, K. G. Winkels, I. R. Peters, D. van der Meer, C. Sun, J. H. Snoeijer, and D. Lohse, "Maximal air bubble entrainment at liquid-drop impact", Phys. Rev. Lett 109, 264501 (2012), See Chapter 2 of this thesis.

[15] E. Klaseboer, R. Manica, and D. Y. C. Chan, "Universal behavior of the initial stage of drop impact", Phys. Rev. Lett 113, 194501 (2014).

[16] S. G. Yiantsios and R. H. Davis, "On the buoyancy-driven motion of a drop towards a rigid surface or a deformable interface", J. Fluid Mech. 217, 547-573 (1990).

[17] P. D. Hicks and R. Purvis, "Air cushioning in droplet impacts with liquid layers and other droplets", Phys. Fluids 23, 062104 (2011).

[18] S. T. Thoroddsen, M.-J. Thoraval, K. Takehara, and T. G. Etoh, "Microbubble morphologies following drop impacts onto a pool surface", J. Fluid Mech. 708, 469-479 (2012).

[19] T. Tran, H. de Maleprade, C. Sun, and D. Lohse, "Air entrainment during impact of droplets on liquid surfaces", J. Fluid Mech. 726, R3 (2013).

[20] S. K. Wilson, "A mathematical model for the initial stages of fluid impact in the presence of a cushioning fluid layer", J. Eng. Math. 25, 265-285 (1991).

[21] O. Reynolds, "On the theory of lubrication and its applications to mr. beauchamp tower's experiments, including an experimental determination of the viscosity of olive oil", Phil. Trans. R. Soc. Lond. 177, 157-234 (1886).

[22] H. Lamb, Hydrodynamics, 6th edition (Cambridge University Press) (1957).

[23] R. H. Davis, J.-M. Serayssol, and E. J. Hinch, "The elastohydrodynamic collision of two spheres", J. Fluid Mech. 163, 479-497 (1986).

[24] C. Pozrikidis, Introduction to theoretical and computational fluid dynamics, 1th edition (Oxford University Press) (1997). 
[25] H. N. Oguz and A. Prosperetti, "Dynamics of bubble growth and detachment from a needle", J. Fluid Mech. 257, 111-145 (1993).

[26] R. P. H. M. Bergmann, D. van der Meer, S. Gekle, J. van der Bos, and D. Lohse, "Controlled impact of a disk on a water surface: cavity dynamics", J. Fluid Mech. 633, 381-409 (2009).

[27] J. H. Snoeijer, P. Brunet, and J. Eggers, "Maximum size of drops levitated by an air cushion", Phys. Rev. E 79, 036307 (2009).

[28] W. Bouwhuis, K. G. Winkels, I. R. Peters, P. Brunet, D. van der Meer, and J. H. Snoeijer, "Oscillating and star-shaped drops levitated by an airflow", Phys. Rev. E 88, 023017 (2013),

See Chapter 6 of this thesis.

[29] E. Klaseboer, J. P. Chevaillier, C. Gourdon, and O. Masbernat, "Film drainage between colliding drops at constant approach velocity: experiments and modeling", J. Colloid. Int. Sc. 229(1), 274-285 (2000).

[30] Y. Yoon, M. Borrell, C. C. Park, and L. G. Leal, "Viscosity ratio effects on the coalescence of two equal-sized drops in a two-dimensional linear flow", J. Fluid Mech. 525, 355-379 (2005). 


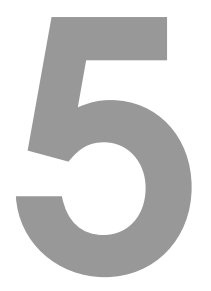

\section{Impact of a high-speed train of microdrops on a liquid pool * $\dagger$}

A train of high-speed microdrops impacting on a liquid pool can create a very deep and narrow cavity, reaching depths more than one thousand times the size of the individual drops. The impact of such a droplet train is studied numerically using Boundary Integral simulations. In these simulations, we solve the potential flow in the pool and in the impacting drops, taking into account the influence of liquid inertia, gravity, and surface tension. We show that for microdrops the cavity shape and maximum depth primarily depend on the balance of inertia and surface tension and discuss how these are influenced by the spacing between the drops in the train. Finally, we derive simple scaling laws for the cavity depth and width.

${ }^{*}$ To be submitted as: W. Bouwhuis, X. Huang, C.U. Chan, P.E. Frommhold, C.D. Ohl, D. Lohse, J.H. Snoeijer, D. van der Meer, "Impact of a high-speed train of microdrops on a liquid pool".

${ }^{\dagger}$ The numerical simulations and analytical work in this chapter are part of the present thesis. The experimental work is due to Xin Huang and Chon U Chan. 


\section{$5.1 \quad$ Introduction}

\subsubsection{Background and motivation}

The impact of mm-sized objects on surfaces has been investigated in great detail. Examples are the study of single drop impact onto a liquid pool [1-7] (focusing on typical terminal velocities of order $1 \mathrm{~m} / \mathrm{s}$ ), multiple drop impact on a pool [8] or impact of a solid object onto a pool [9-12]. Another example is the impact of a continuous water mass, thus a liquid column or a jet, onto a pool [13-19]. These studies focused on the bubbles resulting from the hydrostatic collapse of the generated cavity. Only recently the focus shifted to the impact of much smaller drops, with a radius of a few tens of micrometers, corresponding to the typical size of drops coming from, e.g., an inkjet nozzle [20, 21]. Microdrop impact is highly relevant for many rapidly developing applications, such as immersion lithography [22], extreme ultraviolet (EUV) lithography [23], and 3D-printing [24, 25], spray painting, and spray coating. Very recently it has been found that the phenomena for microdrops impacting on a solid surface are similar to those of larger (mm-sized) impacting drops [26]. In contrast, for impact on a pool on these small length scales, capillary effects are expected to be much more significant when compared to the impact of mm-sized drops, or even dominating over gravity [27]. Capillary effects on the small air bubbles resulting from the air-film rupture have been investigated [7, 28-30], but air bubbles due to the collision of surface waves within the cavity (called 'regular bubble entrapment', as described in Ref. [1-4] for mm-sized drops) for single impacting microdrops is still ongoing research. Impacting microjets and microdrop trains on liquid pools have not yet been studied in detail.

In this chapter, we will look to the impact of a high-speed train of uniformly distributed microdrops on a deep liquid pool. In general, the high-energy impact onto a pool creates a deep cavity (Fig. 5.1a). We will focus on the shape and collapse of the cavities as a function of the relevant impact parameters (drop size, velocity, frequency). By performing Boundary Integral (BI) simulations, where we treat the liquid within the pool and within the impacting drop as potential flow $[12,28]$, the independent modification of these parameters is much more easy than in the experiments (see Sec. 5.1.2). We subsequently connect the numerical results to simple theoretical analysis from which we deduce scaling laws, revealing the key features of the cavity dynamics. Our work thus forms a major step towards understanding multiple drop impact, which plays a role in all the industrial applications mentioned above. 
(a)

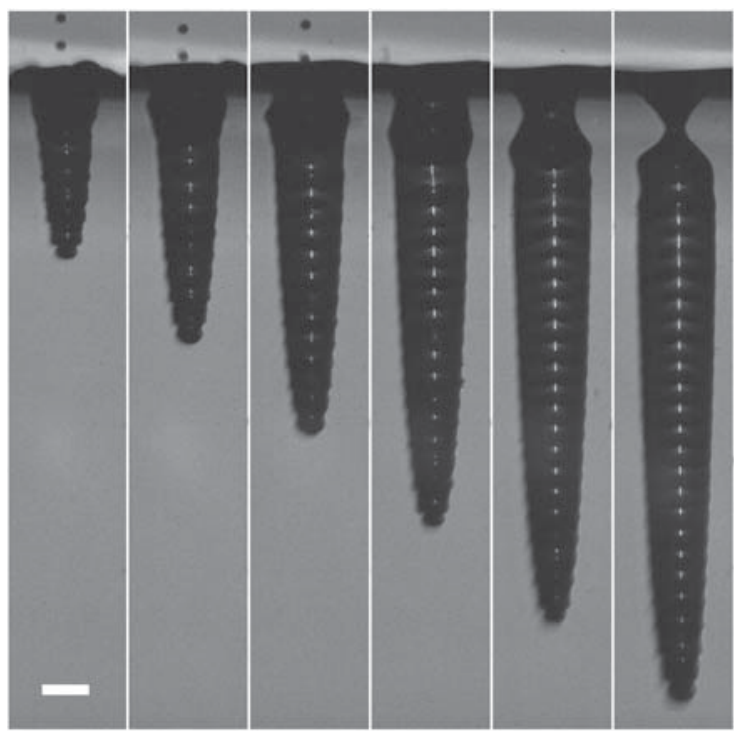

(b)

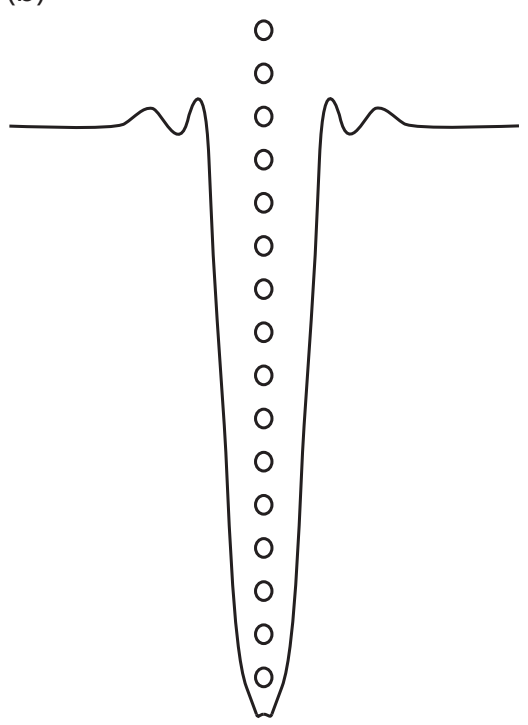

Figure 5.1: (a) Experimental snapshots of a cavity created by a train of $\sim 40 \mu \mathrm{m}$ diameter water drops, which impacts with $\sim 30 \mathrm{~m} / \mathrm{s}$ on a deep pool of water. The scale bar is $200 \mu \mathrm{m}$, and the interframe time is $40 \mu \mathrm{s}$. The cavity reaches a depth of order one thousand times the size of a single drop. (b) Sketch of the impacting train of microdrops.

\subsubsection{Experiments: the creation and impact of a high velocity microdrop train}

Here we briefly describe the experiments reported in Fig. 5.1. More details of the set-up can be found in Refs. [26, 31-34]. A mono-disperse train of droplets is generated at the exit of $20 \mu \mathrm{m}$ diameter nozzle brought into mild vibrations with a piezoceramic transducer. The nozzle is fed from a reservoir filled with deionized water and pressurized at 8 bar using a nitrogen bottle. Applying about $70 \mathrm{~V}$ at $100-400 \mathrm{kHz}$ to the transducer perturbs the thin jet exiting the nozzle and leads to its regular break up due to a RayleighPlateau instability. Figure 5.1a) shows an experimental result for a frequency of $290 \mathrm{kHz}$, which gives a temporal droplet spacing of $\sim 3.5 \mu \mathrm{s}$, a velocity of $\sim 30 \mathrm{~m} / \mathrm{s}$, and a diameter of $\sim 40 \mu \mathrm{m}$. The length of the train (number of drops) can be controlled by two electric fields. Briefly, the droplet train first passes 
through a metallic aperture. By applying a short burst of $800 \mathrm{~V}$, individual and multiple droplets can be charged. Then the droplets pass through a pair of deflection plates, which is held at a constant electric field of $\sim 1.5 \mathrm{MV} / \mathrm{m}$. The undeflected/uncharged droplets are collected, while the deflected droplets pass and impact onto the pool.

In the experimental time sequence of Fig. 5.1a, one recognizes the typical deep and narrow cavity shape, on top of which one can discern a wavy pattern at the lower end of the cavity, caused by the subsequent impact of the individual drops, as indicated in the sketch of the phenomenon provided in Fig. 5.1b. The downward growth speed of the cavity is approximately constant during the impact of the drop train. The collapse of the cavity typically occurs at the top, near the pool surface.

\subsubsection{Objectives}

This chapter will focus on several key questions: What is the shape of the cavity and does there exist a similarity profile? What is the dominant collapse mechanism and what is the depth of the cavity at the time of its collapse? Finally, we will demonstrate the role played by the drop frequency and spacing in the answers to these questions.

The chapter is structured as follows: In Sec. 5.2, we will introduce the problem, the assumptions we make in the simulations and in our theoretical modeling, as well as some details of the numerical method. In Sec. 5.3, we discuss the results of our simulations, and develop a scaling theory to account for these results. Finally, in Sec. 5.4, we conclude, discuss the limitations of our predictions, and give suggestions for future work.

\subsection{Problem statement and numerical method}

In this section, we formulate the problem and conduct a parameter analysis for the typical orders of magnitude in the experiments described in the Introduction (Sec. 5.2.1), and show the numerical method (Sec. 5.2.2).

\subsubsection{Parameters and assumptions}

The geometry of the problem is sketched in Fig. 5.2. We assume cylindrical coordinates $(\vec{r}, \vec{z})$, with $\vec{z}$ pointing upwards and $z=0$ located at the undisturbed surface. In the numerical simulations and in our theoretical analysis we will assume a mono-disperse axisymmetric drop train which consists of drops 


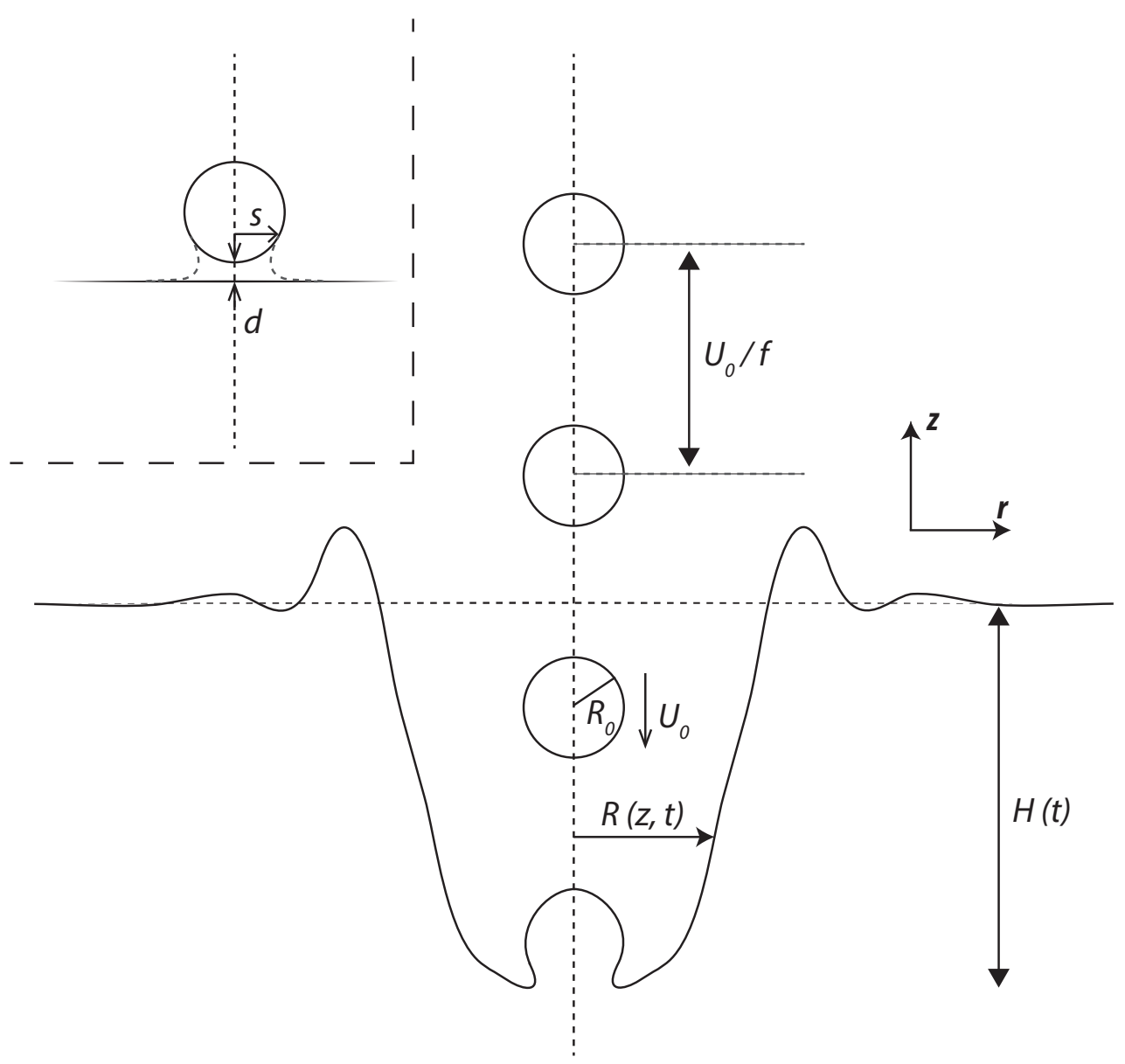

Figure 5.2: Definition sketch of the parameters. The drop train that constists of drops with radius $R_{0}$ and downward velocity $U_{0}$ impacts vertically on a pool surface $(z=0)$. The drop frequency is $f$, and the distance between the drops is $U_{0} / f$. The resulting cavity has a width $R(z, t)$ and a depth $H(t)$, where $t$ is time (from the first impact). We assume axisymmetry. Inset: numerical recombination; at the instant at which the (minimum) gap height between the pool and the drop surface becomes (smaller than) a distance $d$, we recombine the drop surface and the pool surface by connecting the pool curve and the drop curve on a radial distance $s$. 
falling down at a frequency $f$. The drops have radius $R_{0}$ and velocity $U_{0}$, and impact on an initially quiescent pool surface. The liquid of both the pool and the drops is water, with density $\rho=998.1 \mathrm{~kg} / \mathrm{m}^{3}$ and air-liquid surface tension $\gamma=72.8 \mathrm{mN} / \mathrm{m}$. The multiple drop impact on the pool results into a cavity with a time-dependent central depth $H(t)$, where $t$ is the time from the first impact $(H(0)=0)$. The depth- and time-dependent radius of the cavity is $R(z, t)$.

We can express the control parameters in dimensionless form as the Weber number We $=\rho R_{0} U_{0}^{2} / \gamma$ and the ratio $\tilde{f}$ between the drop diameter $2 R_{0}$ and the center-to-center spacing $U_{0} / f$ in between successive drops, i.e., $\widetilde{f}=2 f R_{0} / U_{0}$. The limit $\tilde{f} \rightarrow 0$ is the limit of 'successive' single-dropimpacts: in this limit, the cavity will fully collapse, and the pool surface will re-equilibrate and become flat again, before the next drop impacts. The other limit is $\tilde{f} \rightarrow 1$, which is the theoretical maximum frequency (if $\tilde{f}>1$, the drops would overlap). In the limit $\widetilde{f}=1$, the supply of downward momentum onto the pool is continuous, which is reminiscent of an impacting jet. One could also call $\tilde{f}$ a Strouhal number, comparing the time needed for the drop to move over its own diameter and the time between two subsequent impacts. In the experiments, $\tilde{f}$ is of course smaller than 1 , but still typically of order 1 ; the spacing between the drops is approximately equal to the drop size.

Further important dimensionless parameters of the system are the Reynolds number of the liquid, $\operatorname{Re}=\rho R_{0} U_{0} / \eta$ (where $\eta$ is the dynamic viscosity of the liquid), which is of order 1000 and the Bond number Bo $=\rho g R_{0}^{2} / \gamma$ (where $g$ is the acceleration by gravity), which is of order $10^{-3}$. This implies, respectively, that the viscosity of the liquid can be neglected, and that the effect of surface tension dominates over the effect of gravity. The latter should be contrasted to the plunging disks studied in Ref. [9-12] and the impacting water masses studied in Ref. [14], where the collapse of the cavity was mainly determined by the hydrostatic pressure. One can also express the effect of gravity in terms of the Froude number $\mathrm{Fr}=U_{0}^{2} /\left(g R_{0}\right)$. Typically we have Fr $\sim 10^{5}$, such that we can neglect the effect of gravity during the trajectory of the drops. Since the Reynolds number, the Froude number, and the Weber number are all much larger than 1, we conclude that the behavior of the cavity is mainly determined by inertial effects: the cavity collapse occurs at large times. The cavities created by microdrop train impact can thus indeed grow enormously deep, relative to the size of the drops.

Although the Bond number of a single impacting drop may be small, the Bond number based on the length scale of a deep cavity, $\mathrm{Bo}^{\prime}=\rho g R_{0} H / \gamma$, will 
be a lot larger, such that the hydrostatic pressure becomes non-negligible at the latter stages of the cavity development [27]. A hydrostatic collapse is a 'deep collapse', in the sense that the cavity walls touch the symmetry-axis far below the surface of the pool - a mechanism completely different from a surface tension driven cavity collapse. In our analysis, we will at first instance neglect the effect of hydrostatics, because the collapse of the cavity will be initiated by surface tension, as concluded from the above dimensional analysis, which determines the time scales of the collapse. Note that in the Boundary Integral simulations, we can easily switch on and off the effect of hydrostatics, which is how we confirmed these statements.

We do not simulate the airflow inside the cavity resulting from the movement of both the drop train and the cavity walls. This implies, firstly, that we neglect any small bubble entrapment caused by the increased gas pressure between the pool and the lowest drop, which is a valid assumption, as the length scale of the air bubble entrapment is much smaller than the drop size $[7,28,30]$ and will not influence the flow dynamics on the length scale of the cavity. Secondly, the neglect of the airflow implies that the spherical drops within the train all fall down undecelerated and undeformed (as depicted in Fig. 5.2). It appeared from the experiments that this is a reasonable assumption to implement, except for at the beginning and the end of the drop train. We will come back to these effects at Sec. 5.3.1.

\subsubsection{Numerical method}

\section{Boundary Integral simulations}

The liquid within the pool is treated as incompressible, irrotational, and inviscid. The Laplace equation, $\nabla^{2} \phi=0$, for the flow potential $\phi$, is solved along the liquid interface, using the axisymmetric Boundary Integral (BI) method $[7,10,12,14,28,30,35-37]$. The dynamic boundary condition at the interface is the unsteady Bernoulli equation, which includes both hydrostatic pressure and surface tension. Gravity can easily be switched off; the effect of surface tension can also be switched off, in principle, but in that case one needs a strong artificial smoothing (regridding) procedure, because local strong curvatures and movements will be undamped [36]. The node distribution and time steps vary during the simulation as a function of the instantaneous local curvatures and velocities. The total number of nodes along the pool and cavity surface varies between $\sim 50$ for a flat pool and $\sim 600$ for a deep cavity. The simulation is stopped at the time when the cavity walls touch the axis of sym- 
metry, i.e., we do not simulate the collapse of the cavity and the oscillations of the enormous bubble.

In practice, we only need to simulate the lowest drop of the train as it coalesces with the pool. As soon as the drop and the pool touch, we recombine the corresponding drop and the pool, and add the next drop, above the just reconnected drop, on a distance $U_{0} / f$ (see Fig. 5.2).

\section{The coalescence}

One subtlety remains in the recombination, namely the successive coalescence events between the lowest drop of the train and the pool. Simulating the coalescence stage is a fundamental numerical problem if one assumes potential flow liquids and treats the air as a void [38-41]. More specifically, the inertial coalescence dynamics lead to (successive) bubble entrapment, and from the instant that the interface above the gap and the interface below the gap touch each other (after reconnection), numerical instabilities can not be excluded and further results depend on the local node spacing. However, since we focus on the behavior on the length scale of the cavity, we can choose a recombination threshold distance $d$; as soon as the (minimum) gap height becomes smaller than $d$, we connect the instantaneous pool and lowest drop curve. We also choose a radial cutoff distance $s$; smaller radial distances will be defined to belong to the liquid phase after the reconnection (see inset of Fig. 5.2 for a clarification of both $d$ and $s$ ). At the time of reconnection, two interfaces which were initially disjoint are merged, which implies that we have to subtract the difference in potentials between the most inner node on the former pool surface and the most inner node on the former drop bottom surface. Further, the large local curvatures which might result from the reconnection are immediately smoothed by the local effect of surface tension, such that the interface shape of the reconnected pool and drop indeed looks as what is suggested by the inset of Fig. 5.2.

We have confirmed convergence of the results as a function of both $d$ and $s$, and as long as $d \ll R_{0}$ and $s \gtrsim R_{0} / 2$, we found that our results are independent of these parameters on the length scale of the drops. In our simulations, we choose $d=R_{0} / 10$. Note that if we choose $d$ much smaller, this decreases the minimal numerical time step to a value less than $d / U_{0}$, during the final stages before the defined touch-down, which strongly increases the simulation run-time without adding any valuable information.

Note that, independently of the convergence for varying recombination threshold $d$ and radial cutoff distance $s$ (within reasonable limitations), $\widetilde{f} \rightarrow 1$ 
always remains a special limit, because in the case $\tilde{f}=1$, the drops touch each other. Numerically, it implies that at every single iteration a drop will merge with a next drop. This is why we will not include simulations for $\widetilde{f}$ larger than about 0.7 , for which we will show to already recover the results reminiscent from a continuously impacting jet [14].

\section{$5.3 \quad$ Results}

We will now present our BI simulation results and reveal the key features of the cavity dynamics. Section 5.3.1 contains a quantitative comparison between our numerical results and the experimental results; in Sec. 5.3.2 we turn to the shape of the cavity, and Sec. 5.3.3 describes the cavity dynamics, and will provide an answer to the question of how deep the cavity can become. Our numerical results are complemented by scaling arguments.

\subsubsection{Comparison to experiments}

To quantitatively compare the experimental and numerical cavity contours, Fig. 5.3 shows a time sequence of snapshots during the formation and collapse of the cavity, for the parameters $R_{0}=20 \mu \mathrm{m}, U_{0}=26.75 \mathrm{~m} / \mathrm{s}$, and $f=$ $290 \mathrm{kHz}$. This comparison has been applied for a finite drop train which contains about 85 drops. To do a good comparison for the deep cavities also, the influence of hydrostatics is also involved in the simulations (which only makes a difference in the latter stages, see Sec. 5.2.1). The red lines represent the experimental results; the superimposed blue lines show the results of the BI simulation. The agreement between the experiment and the simulations is very good up to about $0.275 \mathrm{~ms}$. After that, we observe substantial differences, though the downward growth speed of the cavity and the depth of the cavity at the time of its collapse continue to match very well. This is further illustrated in Fig. 5.4, where we plot the cavity height versus time.

However, there are also significant differences between the experimental and numerical results. First of all, the red dots in Fig. 5.3 reveal that the drop train in the experiments was not as uniformly aligned as implemented in the numerical simulations. In particular, in the beginning (lowest part) of the train, drops were bunched up and were slowed down with respect to the 'average' velocity of the train, whereas at the top of the train, the spacing in between the drops was found to be increased. These effects are caused by the drag induced by the air which surrounds the falling drops. This is why the 

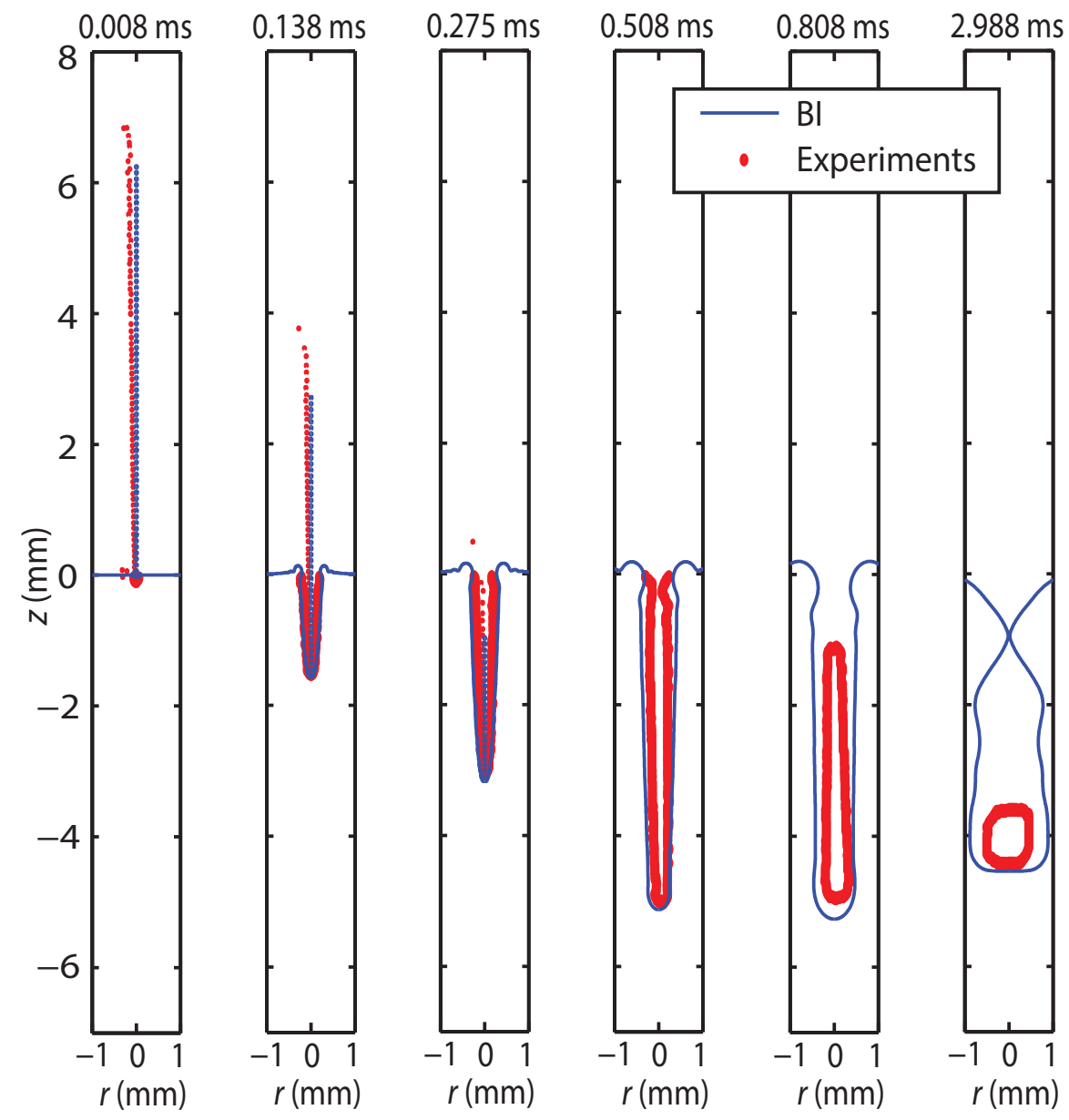

Figure 5.3: Direct comparison between experimental and numerical (BI) results of the cavity contours, for drop radius $R_{0}=20 \mu \mathrm{m}$, impact velocity $U_{0}=26.75 \mathrm{~m} / \mathrm{s}$, and frequency $f=290 \mathrm{kHz}$. The downward growth speed of the cavity $U_{c}$ and the final depth $H_{m}$ match very well (also see Fig. 5.4), but there are also differences between the experiments and the simulations. The most obvious difference is that after $t \sim 0.275 \mathrm{~ms}$, the width of the cavity in the experiments is significantly smaller than the cavity width resulting from simulations, and, related to this, the collapse of the cavity occurs much faster in the experiments than in the numerical simulations. We attribute this to the influence of the streaming air. 


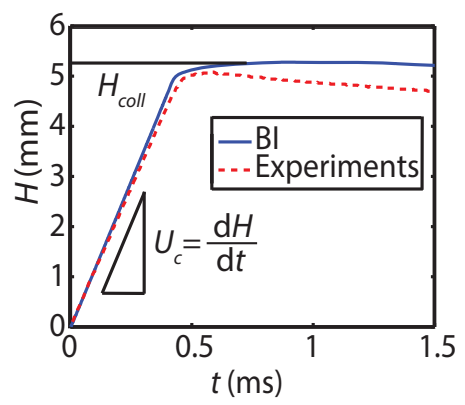

Figure 5.4: Direct comparison between experimental and numerical (BI) results of the cavity depth versus time, for drop radius $R_{0}=20 \mu \mathrm{m}$, impact velocity $U_{0}=26.75 \mathrm{~m} / \mathrm{s}$, and frequency $f=290 \mathrm{kHz}$ (also see Fig. 5.3). The downward growth speed of the cavity $U_{c}$ and the final depth $H_{m}$ match very well.

last part of the train in experiments does not have a significant influence on the formed cavity, and we account for this by a decreased number of drops in the simulated drop train (70 instead of $\sim 85$ ). For the same reason, we are only able to fairly compare experiments and simulations for rather long drop trains, such that the mentioned effects are the least pronounced.

Secondly, it can be observed that the width of the cavity in the experiments is significantly smaller than the cavity width in the simulations, particularly at later times. This effect also leads to a somewhat earlier collapse of the cavity in the experiments compared to the simulations, and the slow decrease of $H$ over time after the end of the drop train $(t \sim 0.5 \mathrm{~ms})$ for the experimental result (red line) shown in Fig. 5.4. We mainly attribute these effects to the influence of the streaming air within the cavity. Here it is good to note that for the case of a disk plunging onto a pool, where the influence of air was included in the BI simulations, the effect of the airflow was only visible for the very final stages of the cavity collapse $[11,12]$. It is however plausible that the small length scale of our problem, together with the presence of the high-speed falling drops within the cavity, significantly increase the influence of the air. Namely, if we assume that the air flows with the speed of the falling drops, $26.75 \mathrm{~m} / \mathrm{s}$, this results in a Reynolds number of the gas $\operatorname{Re}_{g}=\rho_{g} R_{0} U_{0} / \eta_{g}$ of order 10-100. Here, $\rho_{g}$ and $\eta_{g}$ are the density and viscosity of air, respectively, $1.204 \mathrm{~kg} / \mathrm{m}^{3}$, and $1.82 \cdot 10^{-5} \mathrm{~Pa}$ s. Thus, neglecting the effects of the air viscosity, the dynamic pressure drop with respect to the pressure at the cavity 
walls is of order $\rho_{g} U_{0}^{2} \sim 1000 \mathrm{~Pa}$. This pressure drop sucks in the liquid with $\rho_{g} U_{0}^{2} \sim \rho U_{l}^{2}$, where $U_{l}$ is the induced inward speed of the liquid cavity walls, which gives an estimation for the liquid velocity of order $1 \mathrm{~m} / \mathrm{s}$. Since the process of cavity formation takes $\sim 1 \mathrm{~ms}$, the deviation of the cavity shape is expected to be of order $1 \mathrm{~mm}$, which is indeed consistent with the difference between experiment and simulation. Also consistent with this reasoning is that the width of the experimental cavity and that resulting from the simulations deviate the most at the top of the cavity, where the suction of the flowing air has had its influence for the largest time interval. Note, once again, that this deviation is particularly visible in the latter stages, after $t \sim 0.275 \mathrm{~ms}$. In the beginning of the cavity development, experiment and simulation agree.

\subsubsection{Cavity shape}

The robustness of the slender shape of the cavity is evidenced by Fig. 5.5, which contains two more time sequences resulting from the BI simulations, now for two different Weber numbers. Here we used $R_{0}=100 \mu \mathrm{m}, U_{0}=8.5 \mathrm{~m} / \mathrm{s}$ and $85.4 \mathrm{~m} / \mathrm{s}$, and $f=30 \mathrm{kHz}$, corresponding to We $\sim 100$ and 10000 , and $\widetilde{f}=0.70$ and 0.07 , respectively.

Figure 5.6a is a doubly logarithmic plot of the resulting cavity shapes for three different simulations with $\mathrm{We}=100,1000$ and 10000 (including those of Fig. 5.5). Figure 5.6a shows that for large enough Weber number, the cavity profile is parabolic: $R \sim(z+H)^{1 / 2}$, where we corrected for the instantaneous depth of the cavity: $(z+H)$ is the vertical coordinate measured from the bottom at $z=-H(t)$. This parabolic similarity profile is valid between the lower part of the cavity, where there are traveling surface waves resulting from the separate drop-pool collisions, and the top part of the cavity, where the surface tension driven collapse takes place (as we will discuss in Sec. 5.3.3). The larger the Weber number, the more clearly the parabolic shape can be recognized - for the smallest Weber number plotted, the asymptotic stage is not reached, because surface tension makes the collapse behavior at the top of the cavity too dominant. Fig. 5.6b shows the profiles for We $=10000$ at three different times. Together, the panels of Fig. 5.6 show that the parabolic profile can be observed for large enough Weber number and at sufficiently long time after the first impact.

We can understand the parabolic shape of the cavity as follows. Since the cavity has a slender geometry, the fluid flow is predominantly oriented in horizontal slices. Hence, we can apply the two-dimensional Rayleigh equation in cylindrical coordinates. Neglecting the influence of hydrostatics, one 


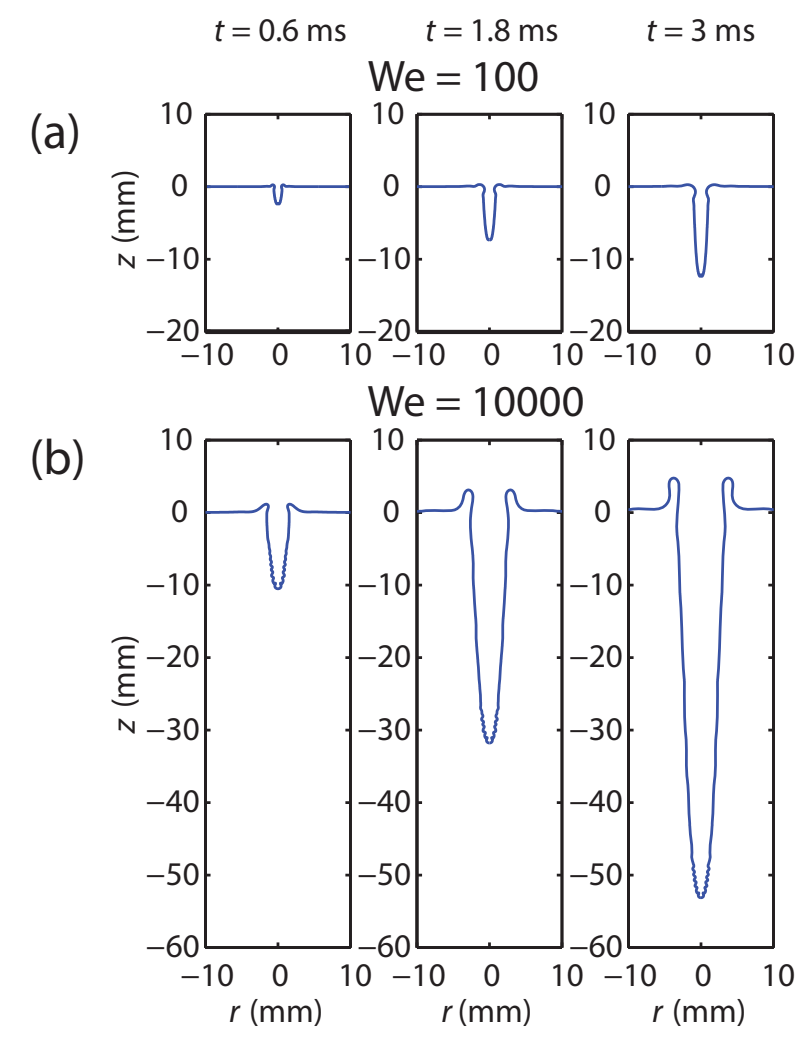

Figure 5.5: Time sequences from BI simulations for two different Weber numbers: (a) $\mathrm{We}=\rho R_{0} U_{0}^{2} / \gamma=100, \tilde{f}=0.7$ (for $U_{0}=85 \mathrm{~m} / \mathrm{s}$ ), and (b) $\mathrm{We}=1000, \tilde{f}=0.07$ (for $U_{0}=8.5 \mathrm{~m} / \mathrm{s}$ ) (in both cases $R_{0}=100 \mu \mathrm{m}$ and $f=30 \mathrm{kHz}$ ). The profiles are shown at times $t=0.6 \mathrm{~ms}, 1.8 \mathrm{~ms}$, and $3 \mathrm{~ms}$. Obviously, the larger velocity drops create a cavity with larger depth and width. 
(a)

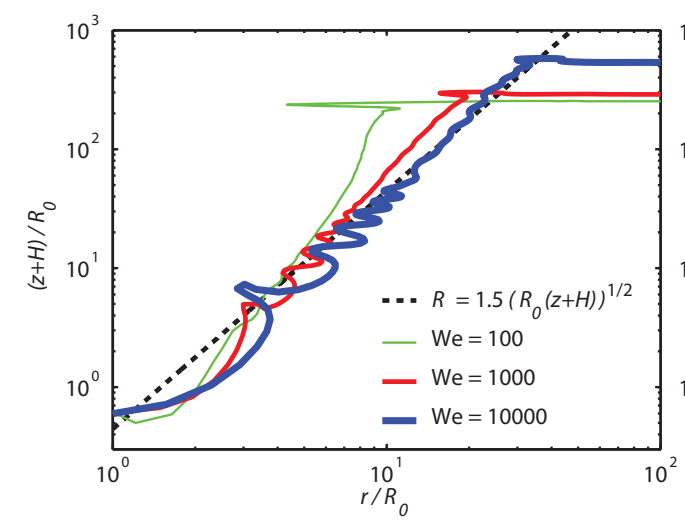

(b)

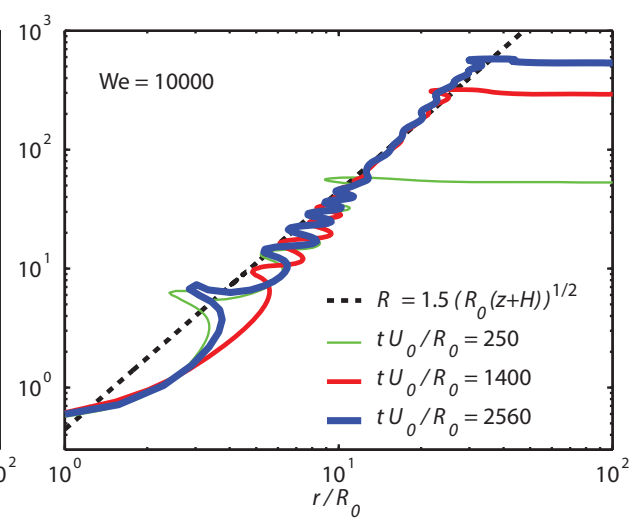

Figure 5.6: Cavity shapes for several Weber numbers $\mathrm{We}=\rho R_{0} U_{0}^{2} / \gamma$ at several times after the first impact $(t=0)$, shown on doubly logarithmic scale. (a) $U_{0}=8.5 \mathrm{~m} / \mathrm{s}, 27.0 \mathrm{~m} / \mathrm{s}$, and $85.4 \mathrm{~m} / \mathrm{s}$ (different times). (b): We $=10000$ at three different times. For both panels $R_{0}=100 \mathrm{~m} / \mathrm{s}$ and $f=30 \mathrm{kHz}$. We find that for large Weber-number and large times after the first impact, the cavity shape approaches a parabola, i.e. $(z(r)+H) \sim r^{2}$. For smaller Weber-numbers this asymptotic stage is not reached, because surface tension makes the collapse behavior at the top of the cavity too dominant.

obtains $[9,42]$ :

$$
\left(R \frac{d^{2} R}{d t^{2}}+\left(\frac{d R}{d t}\right)^{2}\right) \log \left(\frac{R}{R_{\infty}}\right)+\frac{1}{2}\left(\frac{d R}{d t}\right)^{2}=-\frac{\gamma \kappa}{\rho} \approx \frac{\gamma}{\rho R}
$$

where $R_{\infty}$ is an external length scale of the problem that has a weak (logarithmic) influence on the analysis below [9, 11, 42, 43]. In the last approximation we neglected the axial contribution to the curvature; the axial curvature is much smaller than the radial curvature due to the slenderness of the cavity. At large We, we may neglect the right hand side during the first instances of the cavity formation, i.e. at small distance $z+H$. Hence, since $R_{\infty} \gg R$, the dynamics are determined by $R \frac{d^{2} R}{d t^{2}}+\left(\frac{d R}{d t}\right)^{2} \approx 0$, which has the solution 
$R(t) \sim\left(t-t_{0}\right)^{1 / 2}$, where $t=H / U_{c}$ is the time after the first impact on the initially flat pool $(t=0)$ at which the cavity reaches depth $H, t_{0}=-z / U_{c}$ (recall that $z$ is negative below the pool surface), and $U_{c}=d H / d t$ is the (constant) downward growth speed of the cavity. This implies

$$
R(z, t) \sim \sqrt{R_{0}(z+H)}=\sqrt{R_{0}\left(z+U_{c} t\right)}
$$

This is the similarity profile, to which the curves of Fig. 5.6 converge, for large Weber number, sufficiently long after the first impact, and away from the bottom and top of the cavity. The dashed line represents the prediction (5.2), with a fitted prefactor of 1.5.

\subsubsection{Cavity dynamics}

Two important questions arise from the preceding analysis: what is the downward expansion speed of the cavity $U_{c}$ as a function of the impact parameters, and when does surface tension start to act against the expansion of the cavity?

Figure 5.7 shows $U_{c} / U_{0}$ as a function of the ratio $\tilde{f}=2 f R_{0} / U_{0}$. The five different data-sets show the results for five drop frequencies $f=10-30 \mathrm{kHz}$, for varying impact velocities $U_{0}=7-13 \mathrm{~m} / \mathrm{s}$. Thus, the Weber number strongly varies over the data points shown in the figure; every data point belongs to a different set of $(\mathrm{We}, \widetilde{f})$, which is why we choose to mention the dimensional frequency in the legend, while we show dimensionless plots. As explained in Sec. 5.2.2, close to the limit $\widetilde{f} \rightarrow 1$, the number of reconnections per time unit, and thus the relative importance of the corresponding numerical artifacts, is large, which is why we do not show our results for $\widetilde{f}>0$.7. Interestingly, $U_{c} / U_{0}$ converges to $1 / 2$ for $\widetilde{f} \rightarrow 1$. $\widetilde{f}=1$ would be the situation of a drop train with zero spacing, which corresponds to the special case in which momentum is continuously applied to the pool/cavity bottom. This is reminiscent of the continuously impacting jet onto a pool, like described in Ref. [14]. One of the results for the continuously impacting jet scenario described in this work is that the downward expansion speed of the cavity is exactly one half of the impact speed of the jet, $U_{c} / U_{0}=1 / 2$. This ratio $1 / 2$ is indeed approached in our simulations. At smaller frequencies, the net momentum transfer per time unit decreases, and the downward expansion speed of the cavity will thus be smaller, giving a qualitative explanation of our findings in Fig. 5.7.

The Weber number strongly varies over the data in Fig. 5.7, but the relation between $U_{c} / U_{0}$ and $\widetilde{f}$ appears to be universal, in the sense that it is independent of We. The superimposed experimental result, shown by the open 


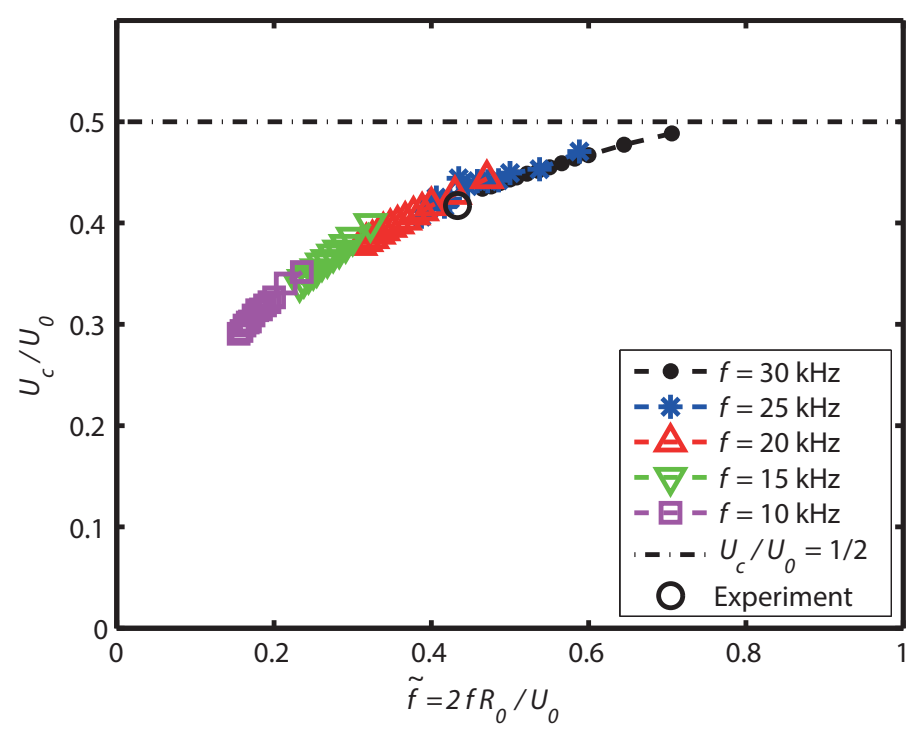

Figure 5.7: The downward cavity growth velocity $U_{c}$ divided by the drop impact speed $U_{0}$ plotted against the ratio $\tilde{f}=2 f R_{0} / U_{0}$ for several values of the Weber number We and the drop frequency $f$. Simulation results for $R_{0}=100 \mu \mathrm{m}$. As long as $f \gg 1 / t_{c}$, which is the case for all these simulations, we find that $U_{c} / U_{0}$ universally depends on $\widetilde{f}$, i.e., $U_{c} / U_{0}$ is independent of We, and converges to $1 / 2$ for $\tilde{f} \rightarrow 1$, where the droplet train turns into a continuous jet. The experimental result, shown by the circle, corresponds to the time sequence of Fig. 5.3, i.e. $R_{0}=20 \mu \mathrm{m}, U_{0}=26.75 \mathrm{~m} / \mathrm{s}$, and $f=290 \mathrm{kHz}$.

circle, corresponds to the time sequence and parameters of Fig. 5.3, where $R_{0}$ was $20 \mu \mathrm{m}$ instead of $100 \mu \mathrm{m}$. This experimental data point is in line with the independence of We. However, this universality will only hold if the collapse time of the small cavity created by the single drop impact is much larger than the time it takes till the next drop impacts, which is generally true for our impact parameters. Otherwise, obviously, since the surface tension influences the local cavity collapse, the relation between $U_{c} / U_{0}$ and $\tilde{f}$ will have a Weber dependence.

With this result of $U_{c}$ as a function of the frequency $\widetilde{f}$, we basically know all the dynamic details of the downward translating parabolic cavity, and we are able to predict its collapse. The time at which surface tension will start 
to pull back the cavity walls can be predicted by solving Eq. (5.1) without neglecting the influence of the surface tension. This can be done analytically, as shown in the appendix, but can also be appreciated from a dimensional argument that will be provided below. For this one must introduce a velocity scale, for which we choose the downward speed of the cavity $U_{c}$ instead of $U_{0}$. This defines a 'modified' Weber number $\mathrm{We}_{m}$ as

$$
\mathrm{We}_{m}=\frac{\rho R_{0} U_{c}^{2}}{\gamma}=\mathrm{We}\left(\frac{U_{c}}{U_{0}}\right)^{2}
$$

and in the remainder of this section we will plot our results as a function of $\mathrm{We}_{m}$.

We will show how the collapse of the cavity depends on $U_{c}$ and the other parameters. For this, we need to separate two cases, which are basically short and long drop trains. More specifically, we distinguish trains for which the collapse of the cavity occurs after the full drop train has impacted, like in our experimental comparison (Figs. 5.3 and 5.4), and trains for which the collapse of the cavity occurs before the end of the drop train impact. In the first case, the cavity depth at the moment of its collapse, $H_{\text {coll }}$, is determined by the number of drops $N$ in the train, $H_{c}=H_{\text {coll }} \sim N U_{c} / f$; in the second case, this depth is determined by the time at which the cavity walls touch the symmetry axis (or drops). Note that in the second case, $H_{c}$ is in general larger than $H_{\text {coll }}$; experiments show that after the cavity walls hit the symmetry axis, the drops within the entrained bubble will still continue impacting on the bubble bottom, an effect that could further increase the depth of the bubble. In that case, to predict $H_{c}$, one needs to take into account the bubble deformation dynamics, which we leave for the moment. Thus, we focus on the prediction of $H_{\text {coll }}$. The time at which surface tension is able to influence the cavity walls, $t_{\text {coll }}$, can be predicted by comparing the dynamic pressure of the radially expanding cavity (which decreases in time, and is the smallest at the top of the cavity, where the parabola that describes it is the widest) and the Young-Laplace pressure based on the azimuthal curvature of the cavity:

$$
\rho\left(\frac{d R_{z=0}}{d t}\right)^{2} \sim \frac{\gamma}{R_{z=0}}
$$

where $R_{z=0}$ is the cavity radius at the pool surface, $z=0$. Substituting the dynamic profile for the cavity found in Sec. 5.3.2, $R_{z=0}(t) \sim \sqrt{R_{0} H(t)}=$ $\sqrt{R_{0} U_{c} t}$, from which $d R_{z=0} / d t(t) \sim \sqrt{R_{0} U_{c} / t}$, gives a prediction of the time at which $R_{z=0}$ reaches the maximum expansion radius of the cavity, which we call $t_{\text {coll }}$ : 


$$
t_{\text {coll }} \sim \frac{\rho^{2} R_{0}^{3} U_{c}^{3}}{\gamma^{2}}=t_{c} \mathrm{We}_{m}^{\frac{3}{2}} .
$$

When we insert $t_{\text {coll }}$ in the similarity profile we can calculate the maximum radius $R_{c}$ of the cavity:

$$
R_{c} \sim \sqrt{R_{0} U_{c} t_{c o l l}} \sim R_{0} \mathrm{We}_{m} .
$$

Combining Eqs. (5.5) and (5.6), we find that the typical time at which the cavity starts to collapse is the capillary time based on the maximum radius of the cavity $\left(t_{\text {coll }} \sim \sqrt{\rho R_{c}^{3} / \gamma}\right)$, as previously found by Ref. [27] for the collapse of a cavity generated by the impact of a solid sphere. The depth of the cavity at the time of its collapse, $H_{\text {coll }}$ can now be predicted by multiplying the expansion velocity by the collapse time:

$$
H_{\text {coll }} \sim U_{c} t_{\text {coll }} \sim U_{c} t_{c} \mathrm{We}_{m}^{\frac{3}{2}} \sim R_{0} \mathrm{We}_{m}^{2} .
$$

From Fig. 5.8a and b, which show the BI simulation results of our parameter scan for $R_{c}$ and $H_{\text {coll }}$, respectively, one can appreciate that the above scaling results agree with the BI results. The curves seem to be universal, independent of the drop frequency $\widetilde{f}$, but note that the $f$-dependence is taken into account by means of $U_{c}$. This confirms that $\mathrm{We}_{m}$, and not We is the proper dimensionless parameter to analyze the maximum cavity radius and the cavity collapse. Note that $\mathrm{We}_{m}$ naturally varies over a smaller range than We, due to its definition (5.3), setting a limit on the range in Fig. 5.8.

\subsection{Discussion}

Summarizing, we found that the expansion of the cavity resulting from a microdrop train impact can be described as a purely inertial mechanism, except for the collapse region that is a result of the capillary forces. From solving the 2D Rayleigh equation, we concluded that the cavity shape is a parabola, which translates downward with a constant velocity $U_{c}$. We showed that $U_{c} / U_{0}$ only depends on the aspect ratio $\widetilde{f}$ of the drop train, i.e., the ratio of drop diameter to drop distance in the train. In addition, we were able to predict the collapse duration $t_{\text {coll }}$, and the cavity depth $H_{\text {coll }}$ at the moment of the collapse.

There are a few limitations of this theory. The most important difference is that the width of the cavity in the experiments, at later times, is much smaller than the width in the numerical simulations, due to, most likely, the 

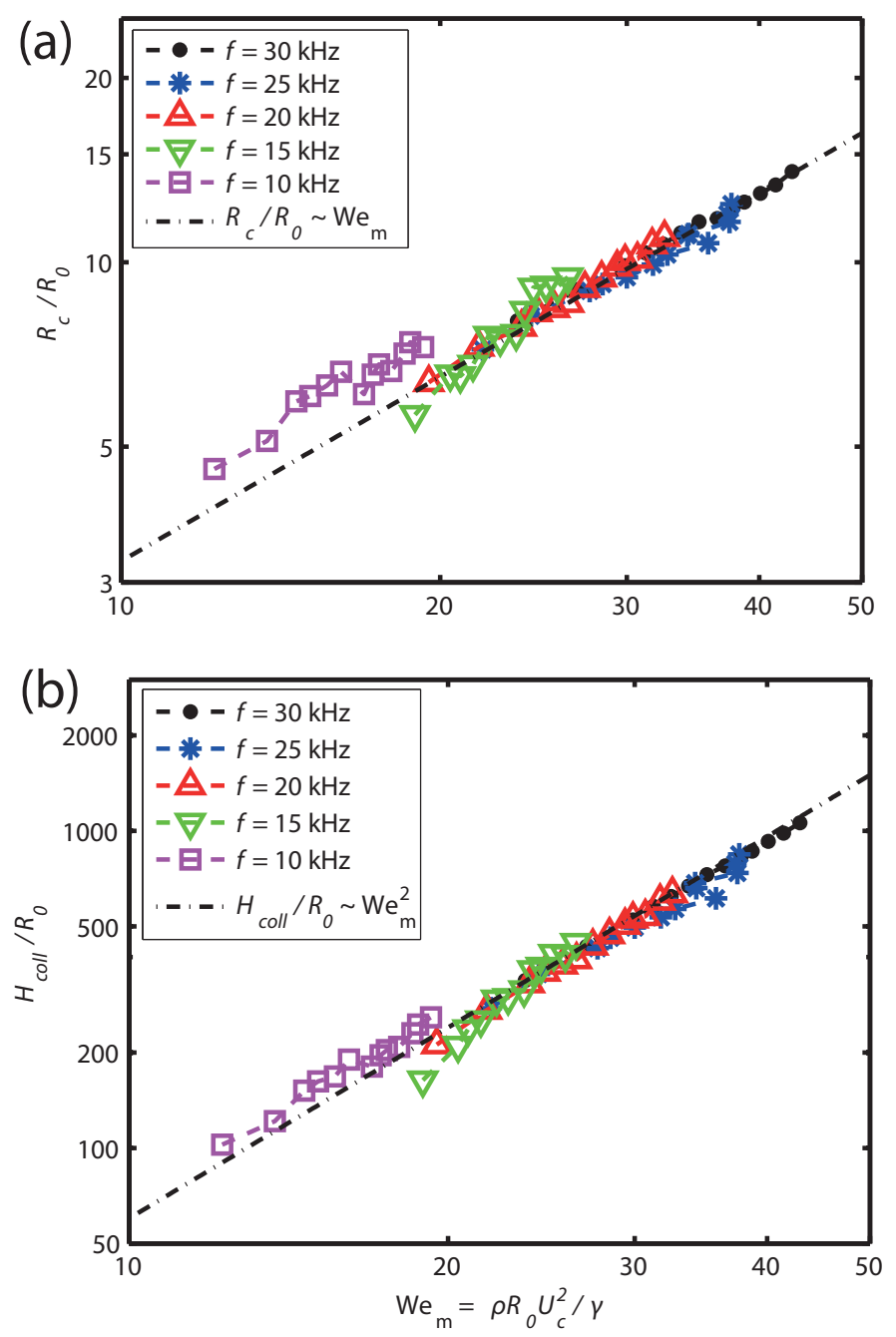

Figure 5.8: (a) Maximum radius of the cavity $R_{c}$, and (b) depth of the cavity $H_{\text {coll }}$ at the moment of its collapse, plotted against the modified Weber number $\mathrm{We}_{m}=\rho R_{0} U_{c}^{2} / \gamma$ based on the vertical cavity growth speed $U_{c}$ for several We and $f . R_{0}=100 \mu \mathrm{m}$. We find a universal behavior with respect to $\mathrm{We}_{m}$, independent of $\tilde{f}$. Superimposed are the scaling predictions: $R_{c} / R_{0} \sim \mathrm{We}_{m}$, and $H_{\text {coll }} / R_{0} \sim \mathrm{We}_{m}^{2}$, with which we find very good agreement. 
influence of the streaming air, which has been neglected. The fact that our predictions for the depth of the cavity are still accurate for the experiment originates from the fact that the dynamics at the bottom of the cavity, in particular $U_{c}$, is hardly influenced by the airflow. Thus, the collapse in experiments occurs earlier than predicted, but after that collapse, the bottom of the cavity keeps expanding with the same $U_{c}$ till the end of the drop train, and the instantaneous depths are predicted correctly, as shown in Fig. 5.4. Our simulation method appears to be a very efficient way to simulate drop train impact, if one focuses on the depth of the cavity, neglecting the influence of air. Since the BI code solves the Laplace equation only along the pool surface we are able to account for the many different length scales in the problem, ranging from the cavity size, through the drop dimension, to the scale of the recombination of the free surfaces of the drop and the cavity. This would pose difficulties for most other simulation methods. However, an advantage of the use of a two-phase simulation method will be the ability to solve correctly for the influence of the local airflow, in particular the airflow induced by the falling drops, which shows possible future work on this subject.

Other possible future work on this subject focuses on different mechanisms of the cavity collapse and bubble formation processes. In fact this has been described in detail in Ref. [27], but this is based on sphere impact on the pool. Our case, the impact of a collection of liquid onto a pool, gives rise to the following question for future research: how does the mass distribution and the time scale of the impact influence the cavity shape, and thus the collapse? We already found that the impact of a jet is from qualitative point of view not different from the impact of a drop train, except that the frequency is an explicit parameter for the latter case. We found that, besides the total amount of volume which impacts onto the pool, the length- and timescale of the total impact is highly determinative for the cavity behavior, because it determines the relevance of hydrostatics vs. surface tension. This is a new aspect with respect to all previous work on impact mentioned in the beginning of the Introduction of this chapter.

\section{A Solution of the cylindrical Rayleigh equation including the influence of surface tension}

Here, we analytically solve Eq. (5.1), including the Young-Laplace pressure based on the azimuthal curvature of the cavity. We start from Eq. (5.1), which we non-dimensionalize using the drop radius $R_{0}$ and $R_{0}$ over the downward 
cavity speed $U_{c}$ as a typical time scale. This gives

$$
\left(\frac{d}{d \widetilde{t}}\left(\widetilde{R} \frac{d \widetilde{R}}{d \widetilde{t}}\right)\right) \log \left(\frac{\widetilde{R}}{\widetilde{R}_{\infty}}\right)+\frac{1}{2}\left(\frac{d \widetilde{R}}{d \widetilde{t}}\right)^{2}=\frac{1}{\mathrm{We}_{m}} \frac{1}{\widetilde{R}},
$$

where $\widetilde{R}=R / R_{0}, \widetilde{R}_{\infty}=R_{\infty} / R_{0}, \widetilde{t}=t U_{c} / R_{0}$, and $\mathrm{We}_{m}=\rho R_{0} U_{c}^{2} / \gamma$. In the remainder of this appendix we will drop the tildes.

Writing $U=d R / d t$ we first note that

$$
\frac{d}{d t}(R U)=U^{2}+R \frac{d U}{d t}=U^{2}+\frac{1}{2} R \frac{d}{d R}\left(U^{2}\right),
$$

where we used that $d U / d t=d U / d R d R / d t=U d U / d R$. With this, Eq. (5.8) becomes:

$$
U^{2} \log \left(\frac{R}{R_{\infty}}\right)+\frac{1}{2} R \frac{d}{d R}\left(U^{2}\right) \log \left(\frac{R}{R_{\infty}}\right)+\frac{1}{2} U^{2}=\frac{1}{\mathrm{We}_{m}} \frac{1}{R}
$$

Now let $x=\frac{R}{R_{\infty}}$ and $y=\left(\frac{d R}{d t}\right)^{2}$. Then the above equation reads

$$
\frac{1}{2} x \log (x) \frac{d y}{d x}+\left(\log (x)+\frac{1}{2}\right) y=\frac{1}{\mathrm{We}_{m}} \frac{1}{R_{\infty} x} .
$$

This is an ordinary linear differential equation of which the solution consists of a homogeneous part and a particular part. The homogeneous solution of $(1 / 2) x \log (x) d y / d x+(\log (x)+1 / 2) y=0$ is $y_{H}(x)=K /\left(x^{2} \log (x)\right)$, where $K$ is an integration constant. A particular solution of the shape $y_{P}(x)=$ $C x^{a} / \log (x)$ gives a solution for $a=-1$ and $C=2 /\left(\mathrm{We}_{m} R_{\infty}\right)$, so the full solution to Eq. (5.11) is:

$$
y(x)=\frac{K}{x^{2} \log (x)}+\frac{2}{\mathrm{We}_{m} R_{\infty} x \log (x)} .
$$

Going back to the original variables we have

$$
\left(\frac{d R}{d t}\right)^{2}=\left(2+\frac{K^{\prime}}{R}\right) \frac{1}{\mathrm{We}_{m} R \log \left(\frac{R}{R_{\infty}}\right)},
$$

with $K^{\prime}=K R_{\infty}^{2} \mathrm{We}_{m}$. If we scale back to the dimensional variables and substitute the initial conditions $R(t=0)=A R_{0}$ and $d R / d t(t=0)=B U_{c}$ (with $\mathrm{A}$ and $\mathrm{B}$ positive real numbers), the solution reads 


$$
\left(\frac{d R}{d t}\right)^{2}=\frac{R_{0} U_{c}^{2}}{R \log \left(\frac{R}{R_{\infty}}\right)}\left[\frac{2}{\mathrm{We}_{m}}+\frac{B^{2} A^{2} R_{0} \log \left(\frac{R_{0}}{R_{\infty}}\right)-\frac{2 A R_{0}}{\mathrm{We}_{m}}}{R}\right]
$$

For $\mathrm{We}_{m} \rightarrow \infty$, this implies

$$
\frac{R \frac{d R}{d t}}{R_{0} U_{c}}=A B \sqrt{\frac{\log \left(\frac{A R_{0}}{R_{\infty}}\right)}{\log \left(\frac{R}{R_{\infty}}\right)}}
$$

which is real and positive as long as $R$ and $R_{0}$ are smaller than $R_{\infty}$.

Finally, to find the maximum expansion radius of the cavity, $R_{c}$, one uses $d R / d t=0$ in Eq. (5.14), which leads to

$$
R_{c}=R_{0}\left(A+\frac{A^{2} B^{2} \mathrm{We}_{m} \log \left(\frac{R_{\infty}}{R_{0}}\right)}{2}\right) .
$$

For large $\mathrm{We}_{m}$, we thus expect $R_{c} \sim R_{0} \mathrm{We}_{m}$, which agrees with the scaling law found on dimensional grounds (Eq. (5.6)).

\section{References}

[1] H. C. Pumphrey and P. A. Elmore, "The entrainment of bubbles by drop impacts", J. Fluid Mech. 220, 539-567 (1990).

[2] A. Prosperetti and H. N. Oguz, "The impact of drops on liquid surfaces and the underwater noise of rain", Ann. Rev. Fluid Mech. 25, 577-602 (1993).

[3] A. Wang, C.-C. Kuan, and P.-H. Tsai, "Do we understand the bubble formation by a single drop impacting upon liquid surface?", Phys. Fluids 25(10), 101702 (2013).

[4] S. Chen and L. Guo, "Viscosity effect on regular air bubble entrapment during drop impact into a deep pool", Chem. Eng. Sci. 109, 1-16 (2014).

[5] S. T. Thoroddsen, M.-J. Thoraval, K. Takehara, and T. G. Etoh, "Microbubble morphologies following drop impacts onto a pool surface", J. Fluid Mech. 708, 469-479 (2012). 
[6] T. Tran, H. de Maleprade, C. Sun, and D. Lohse, "Air entrainment during impact of droplets on liquid surfaces", J. Fluid Mech. 726, R3 (2013).

[7] M. H. W. Hendrix, W. Bouwhuis, D. van der Meer, D. Lohse, and J. H. Snoeijer, "Universal mechanism for air entrainment during liquid impact", (2015), submitted to J. Fluid Mech.

See Chapter 3 of this thesis.

[8] A. G. Bick, W. D. Ristenpart, E. A. van Nierop, and H. A. Stone, "Bubble formation via multidrop impacts", Phys. Fluids 22, 042105 (2010).

[9] R. P. H. M. Bergmann, D. van der Meer, M. A. Stijnman, M. Sandtke, A. Prosperetti, and D. Lohse, "Giant bubble pinch-off", Phys. Rev. Lett. 96, 154505 (2006).

[10] R. P. H. M. Bergmann, D. van der Meer, S. Gekle, J. van der Bos, and D. Lohse, "Controlled impact of a disk on a water surface: cavity dynamics", J. Fluid Mech. 633, 381-409 (2009).

[11] S. Gekle, J. H. Snoeijer, D. Lohse, and D. van der Meer, "Approach to universality in axisymmetric bubble pinch-off", Phys. Rev. E 80, 036305 (2009).

[12] S. Gekle, I. R. Peters, J. M. Gordillo, D. van der Meer, and D. Lohse, "Supersonic airflow due to solid-liquid impact", Phys. Rev. Lett. 104, 024501 (2010).

[13] A. R. Kolaini, R. A. Roy, L. A. Crum, and Y. Mao, "Low-frequency underwater sound generation by impacting transient water jets", J. Acoust. Soc. Am. 94, 2809-2820 (1993).

[14] H. N. Oguz, A. Prosperetti, and A. R. Kolaini, "Air entrapment by a falling water mass", J. Fluid Mech. 294, 181-207 (1995).

[15] C. Clanet and J. C. Lasheras, "Depth of penetration of bubbles entrained by a plunging water jet", Phys. Fluids 9(7), 1864-1866 (1997).

[16] G. J. Storr and M. Behnia, "Experiments with large diameter gravity driven impacting liquid jets", Exp. Fluids 27, 60-69 (1999).

[17] B. Kersten, C. D. Ohl, and A. Prosperetti, "Transient impact of a liquid column on a miscible liquid surface", Phys. Fluids 15, 821-824 (2003). 
[18] W. G. Szymczak, S. L. Means, and J. C. W. Rogers, "Computations of bubble formation and pulsations generated by impacting cylindrical water jets", J. Eng. Math. 48, 375-389 (2004).

[19] X. L. Qu, L. Khezzar, D. Danciu, M. Labois, and D. Lakehal, "Characterization of plunging liquid jets: A combined experimental and numerical investigation", Int. J. Multiphase Flow 37, 722-731 (2011).

[20] O. A. Basaran, H. Gao, and P. P. Bhat, "Nonstandard inkjets", Annu. Rev. Fluid Mech. 45, 85-113 (2013).

[21] T. W. Driessen, R. J. M. Jeurissen, H. Wijshoff, F. Toschi, and D. Lohse, "Stability of viscous long filaments", Phys. Fluids 25, 062109 (2013).

[22] D. L. Keij, K. G. Winkels, H. Castelijns, M. Riepen, and J. H. Snoeijer, "Bubble formation during the collision of a sessile drop with a meniscus", Phys. Fluids 25, 082005 (2013).

[23] A. L. Klein, W. Bouwhuis, C. W. Visser, H. Lhuissier, C. Sun, J. H. Snoeijer, E. Villermaux, D. Lohse, and H. Gelderblom, "Drop shaping by laser-pulse impact", Phys. Rev. Appl. 3, 044018 (2015), See Chapter 7 of this thesis.

[24] I. Gibson, D. W. Rosen, and B. Stucker, Additive manufacturing technologies, 12 edition (Springer) (2010).

[25] R. Pohl, C. W. Visser, G. R. B. E. Römer, D. Lohse, C. Sun, and A. J. Huis in 't Veld, "Ejection regimes in picosecond laser-induced forward transfer of metals", Phys. Rev. Applied 3, 024001 (2015).

[26] C. W. Visser, P. E. Frommhold, S. Wildeman, R. Mettin, D. Lohse, and C. Sun, "Dynamics of high-speed micro-drop impact: numerical simulations and experiments at frame-to-frame times below $100 \mathrm{ns",} \mathrm{Soft} \mathrm{Matter}$ 11(9), 1708-1722 (2015).

[27] J. M. Aristoff and J. W. M. Bush, "Water entry of small hydrophobic spheres", J. Fluid Mech. 93, 45-78 (2009).

[28] W. Bouwhuis, R. C. A. van der Veen, T. Tran, D. L. Keij, K. G. Winkels, I. R. Peters, D. van der Meer, C. Sun, J. H. Snoeijer, and D. Lohse, "Maximal air bubble entrainment at liquid-drop impact", Phys. Rev. Lett 109, 264501 (2012),

See Chapter 2 of this thesis. 
[29] J. S. Lee, B. M. Weon, J. H. Je, and K. Fezzaa, "How does an air film evolve into a bubble during drop impact?", Phys. Rev. Lett. 109, 204501 (2012).

[30] W. Bouwhuis, M. H. W. Hendrix, D. van der Meer, and J. H. Snoeijer, "Initial surface deformations during impact on a liquid pool", J. Fluid Mech. 771, 503-519 (2015),

See Chapter 4 of this thesis.

[31] N. R. Lindblad and J. R. Schneider, "Production of uniform-sized liquid droplets", J. Sci. Instrum. 42, 635-638 (1965).

[32] G. Brenn, "On the controlled production of sprays with discrete polydisperse drop size spectra", Chem. Eng. Sci. 55(22), 5437-5444 (2000).

[33] H. Y. Kim, S. Y. Park, and K. Min, "Imaging the high-speed impact of microdrop on solid surface", Rev. Sci. Instrum. 74(11), 4930-4937 (2003).

[34] P. E. Frommhold, A. Lippert, F. L. Holsteyns, and R. Mettin, "Highspeed monodisperse droplet generation by ultrasonically controlled microjet breakup", Exp. in Fluids 55, 1-12 (2014).

[35] C. Pozrikidis, Introduction to theoretical and computational fluid dynamics, 1th edition (Oxford University Press) (1997).

[36] H. N. Oguz and A. Prosperetti, "Dynamics of bubble growth and detachment from a needle", J. Fluid Mech. 257, 111-145 (1993).

[37] W. Bouwhuis, K. G. Winkels, I. R. Peters, P. Brunet, D. van der Meer, and J. H. Snoeijer, "Oscillating and star-shaped drops levitated by an airflow", Phys. Rev. E 88, 023017 (2013),

See Chapter 6 of this thesis.

[38] J. Eggers, J. R. Lister, and H. A. Stone, "Coalescence of liquid drops", J. Fluid Mech. 401, 293-310 (1999).

[39] M. R. Davidson, "Spreading of an inviscid drop impacting on a liquid film", Chem. Eng. Sc. 57, 3639-3647 (2002).

[40] L. Duchemin, J. Eggers, and C. Josserand, "Inviscid coalescence of drops", J. Fluid Mech. 487, 167-178 (2003). 
[41] J. Billingham and A. C. King, "Surface-tension-driven flow outside a slender wedge with an application to the inviscid coalescence of drops", J. Fluid Mech. 533, 193-221 (2005).

[42] D. Lohse, R. P. H. M. Bergmann, R. Mikkelsen, C. Zeilstra, D. van der Meer, M. Versluis, K. van der Weele, M. A. van der Hoef, and J. A. M. Kuipers, "Impact on soft sand: Void collapse and jet formation", Phys. Rev. Lett. 93, 198003 (2004).

[43] J. Eggers, M. A. Fontelos, D. Leppinen, and J. H. Snoeijer, "Theory of the collapsing axisymmetric cavity", Phys. Rev. Lett. 98, 094502 (2007). 


\section{6 \\ Oscillating and star-shaped drops levitated by an airflow $* \dagger$}

We investigate the spontaneous oscillations of drops levitated above an air cushion, eventually inducing a breaking of axisymmetry and the appearance of 'star drops'. This is strongly reminiscent of the Leidenfrost stars that are observed for drops floating above a hot substrate. The key advantage of this work is that we inject the airflow at a constant rate below the drop, thus eliminating thermal effects. We perform experiments with drops of different viscosities and observe stable states, oscillations and chimney instabilities. We find that for a given drop size the instability appears above a critical flow rate, where the latter is largest for small drops. All these observations are reproduced by numerical simulations, where we treat the drop using potential flow and the gas as a viscous lubrication layer. Our results demonstrate that thermal effects are not important for the formation of star drops, and strongly suggest a purely hydrodynamic mechanism for the formation of Leidenfrost stars.

${ }^{*}$ Published as: W. Bouwhuis, K.G. Winkels, I.R. Peters, P. Brunet, D. van der Meer, J.H. Snoeijer, "Oscillating and star-shaped drops levitated by an airflow", Phys. Rev. E 88, 023017 (2013).

${ }^{\dagger}$ The numerical simulations in this chapter are part of the present thesis. The experimental work is due to Koen Winkels. 


\subsection{Introduction}

Drops of water can levitate above a very hot plate due to the so-called 'Leidenfrost' effect $[1,2]$. In this situation, drops float on a thin layer of water vapor that results from evaporation between the hot substrate and the drop. The shape and dynamics of the vapor layer can be quite complex [3] and can be used to move liquid along a surface with the help of unevenly textured substrates [4-6]. Under some conditions, drops spontaneously start to oscillate and develop 'star-shapes' or 'faceted shapes' [7-11]. Recently, it has been found that this phenomenon occurs not only in the case of Leidenfrost drops, but also for drops levitating on a steady and ascending uniform airflow at room temperature [12]. Figure 6.1 shows examples of levitating star drops obtained with water, taken from Brunet et al., 2011 [12]. The origin of the oscillatory instability has remained unclear, but the striking similarities with the Leidenfrost stars suggest a common mechanism for both, based only on hydrodynamics and free surface dynamics, without invoking any thermal effects.
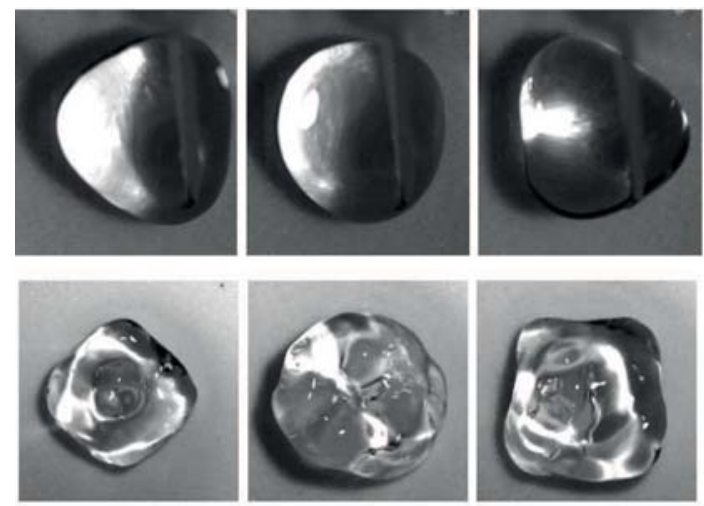

Figure 6.1: Star drops levitated by a steady (i.e., non-pulsating) airflow. Top: mode $n=3$; bottom: mode $n=4$. Figure from Brunet et al., 2011 [12].

Drops with faceted shapes have been observed in various systems with a periodic forcing of frequency close to the eigenmodes of the drop. Such drop shapes arise for drops on vertically vibrated hydrophobic substrates [13, 14], acoustically levitated drops with low-frequency modulated pressure [15], liquid metal drops subjected to an oscillating magnetic field [16], or drops on a pulsating air cushion $[17,18]$. Using simple arguments [19], the appearance of these stars can be explained by the temporal modulation of the eigenfrequency 
of the drop, due to the external forcing, thus inducing a parametric instability. This suggests the following scenario for the formation of stars in a steady ascending airflow: a first instability leads to a vertical oscillation of the drop, which through a secondary, parametric instability leads to the formation of (period doubled) oscillating stars.

Rayleigh and Lamb [20] already predicted that for small enough deformations and for inviscid spherical drops, the resonance frequencies of the drops are given by:

$$
f_{n}=\frac{1}{2 \pi}\left(\frac{n(n-1)(n+2) \gamma}{\rho_{l} R^{3}}\right)^{1 / 2}
$$

where $f_{n}$ stands for the resonance frequency of the $n^{\text {th }}$ mode of oscillation, $R$ is the radius, $\gamma$ and $\rho_{l}$ are the liquid surface tension and density, respectively. When the drop shape is different from the ideal spherical case, the resonance frequencies are modified with much more complex expressions, but in the case of a liquid puddle of radius $R$ much larger than the averaged drop height $H_{d}$, the eigenfrequencies take the following simple expression [19]:

$$
f_{n}=\frac{1}{2 \pi}\left(\frac{n\left(n^{2}-1\right) \gamma}{\rho_{l} R^{3}}\right)^{\frac{1}{2}}
$$

where $n$ is now the number of lobes on the drop in the azimuthal direction. Note that in practice, the frequencies predicted by Eq. (6.1) and (6.2) are very similar. Thus it becomes clear that a parametric instability should occur when the drop radius is modulated in time. The same happens when due to a periodic external forcing, the drop stands in a time-periodic acceleration field. In that case the height of the cylindrical liquid puddle $H_{d}$ also varies periodically, and for a non-wetting condition (contact-angle close to $180^{\circ}$ ) this height is simply equal to twice the effective capillary length $\ell_{c}=\sqrt{\gamma /\left(\rho_{l} a\right)}$, $a$ being the instantaneous acceleration (without forcing, $a$ is equal to the acceleration of gravity $g$ ). By volume conservation, a time dependence of $H_{d}$ results into an oscillation of the radius $R$. Assuming small deformations, $R$ will have the same time-periodicity as the external forcing. Then, star-shaped oscillations by parametric forcing typically display a frequency equal to half of the driving (vertical oscillation) frequency [19].

In the case of a steady, non-pulsating air cushion or Leidenfrost levitation, the key question is to identify the origin of the vertical oscillations: what is the mechanism that induces a time-periodic instability, which in turn gives rise to vertical oscillations of the drop center-of-mass and shape? Once the 
origin of this instability is explained, the appearance of star drops is likely to originate from the parametric instability as stated above. Recent experiments with star drops levitated on a continuous flow air cushion (Fig. 6.1) suggest that these star drops do not result from a temperature gradient-induced instability, contrary to what was previously hypothesized [21]. Apart from the oscillatory instability, a levitated drop can develop a 'chimney', for which an air bubble develops below the drop and pierces the center of the drop [22]. This phenomenon has been explained theoretically from a breakdown of steady solutions $[23,24]$. Interestingly, the numerical simulations for very viscous drops did not display any oscillatory instability. Therefore, the determination of the mechanisms for oscillations requires a more complex numerical scheme than those of Refs. [23, 24].

In this chapter, we experimentally and numerically study drops levitated by an air-cushion, focusing on the instability to chimney formation, oscillations and star drops. The experiments consist of a significantly improved variant of that in Brunet et al., 2011 [12], where we now can determine the threshold of instabilities with good accuracy. For the numerical simulations, the proximity of the cushion to the drop calls for a method capable of accurately describing the gas-liquid interface, which leads us to employing an inviscid Boundary Integral method for the description of the drop. Inspired by the success of lubrication models in providing steady solutions for the drop shape we use a lubrication approximation for the airflow below the drop (Fig. 6.2). This coupling has also been applied to simulate the impact of liquid drops on solid plates, and appeared to be successful in the regimes of both small and large impact velocities (see Chapter 2 of this thesis). The numerical implementation of the drop is completely axisymmetric and aims to explain the appearance of up-down oscillations for the drop.

The chapter is organized as follows: in Sec. 6.2, we present the setup we used to obtain the oscillating levitated drops experimentally, for liquids of different viscosities. Results of these experiments are shown in Sec. 6.3. Then, we describe the numerical scheme in detail (Sec. 6.4), and show the different regimes exhibited by the model (Sec. 6.5). In the last section, we conclude on these results.

\subsection{Experimental setup}

It is well known that in case of Leidenfrost drops, the drops are levitated by a vapor layer. The vapor, coming directly from the drop, generates a cushioning 


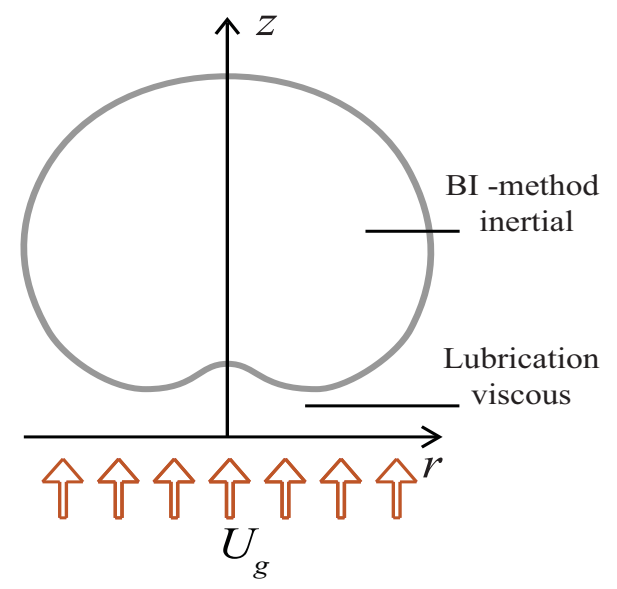

Figure 6.2: Numerical implementation of the drop levitated by an airflow with uniform upward flow velocity $U_{g}$. The numerical simulations consist of a coupling between the Boundary Integral method for the inviscid drop, and the lubrication approximation for the airflow beneath the drop. The flow inside the drop is assumed to be a potential flow; the flow at the bottom of the drop is a viscous flow, in which inertial effects are neglected.

layer for levitation due to the build up of a lubrication pressure between the lower part of the drop and the substrate. To avoid temperature effects and to directly control the gas flux in the layer, another experimental method was introduced in Brunet et al., 2011 [12]. In this experimental method the air cushion is created by an ascending airflow (Fig. 6.3). The airflow is forced through a porous glass medium (Duran Group, Filter Funnel, porosity 3, inner diameter $56 \mathrm{~mm}$ ) that is covered by a coarse grid. The bronze grid is made super-hydrophobic (electroless galvanic deposited metal [26] and humid lowsurface energy molecular deposition) to avoid imbibition of the hydrophilic porous medium. The large pressure load on the porous medium creates an approximately homogeneous outflow, which is assumed to be hardly affected by the small pressure load of the drop. Consequently, if the airflow $Q$ is large enough, a lubricating layer (air cushion) can emerge and support the complete weight of the drop. There exists a threshold drop size $R$ and gas flow rate $Q$ at which the drops become unstable and start to oscillate, i.e., the instability threshold. The airflow is measured with an Aalborg flow meter (range: 0 - 60 liters/min). Since the drop is very mobile in the levitated state, it is necessary to hold it using a needle. This fixates the drop at a constant location on the 
substrate. The same needle is used to supply and subtract liquid from the drop via a syringe. To study the drop behavior for various flow rates $Q$ and drop sizes $R$, the drop motions are recorded from a top view, with a high-speed camera at $1000 \mathrm{fps}$ (Phantom V9). Using a macro lens (Nikon Aspherical Macro, 1:2) with extension tubes, a resolution of $42 \mu \mathrm{m} /$ pixel is obtained (see Fig. 6.3). Reflective illumination (IDT, LED light source) is realized via a 45 degrees tilted beam splitter.

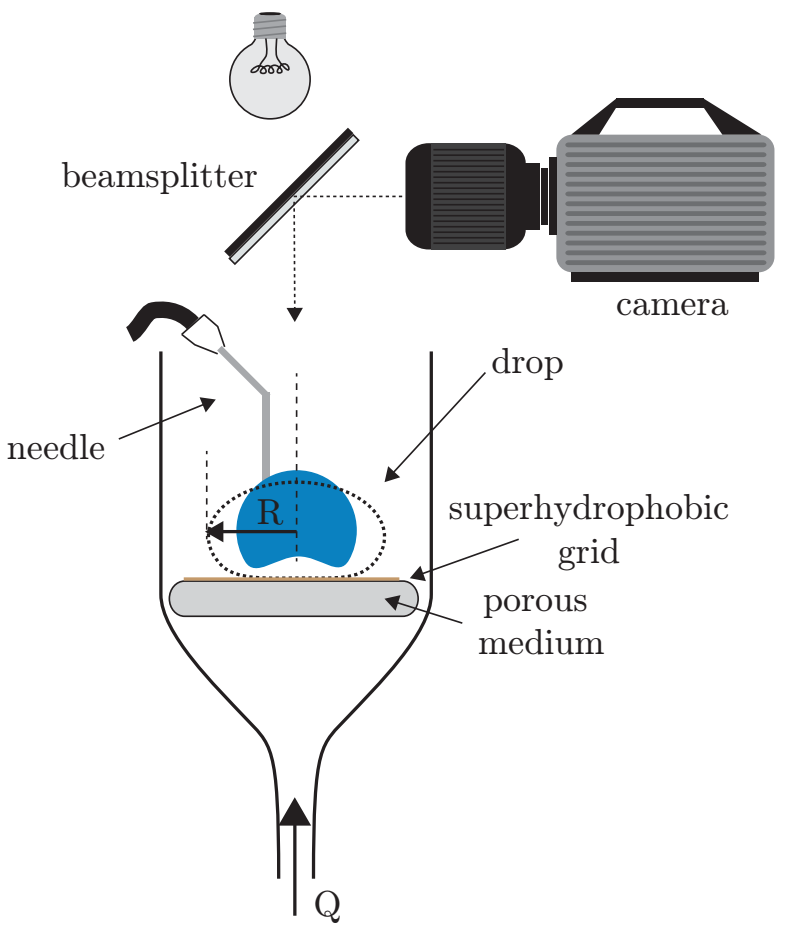

Figure 6.3: Sketch of the experimental setup. Illumination and camera view are obtained using a beamsplitter. A flow rate $Q$ is prescribed through a porous medium. Since the levitated drop is very mobile, it is held in position by a needle, which also supplies the liquid.

The aim of this work is to study the instability threshold (appearance of drop oscillations) for levitated drops. To verify reproducibility of the experiment, each measurement is repeated multiple times and by two different procedures. In the first method, each measurement starts with a new constant flow rate $Q=Q_{t}$ and a small drop size $R$. Then the drop volume is slowly increased by pumping liquid into it. The feeding is continued until the drop 
reaches a floating state $\left(R<R_{t}\right)$ which finally becomes unstable once the drop size equals the threshold size $R_{t}$ for flow rate $Q_{t}$. The volume increase of the drop is directly stopped and subsequently, the dynamics of the unstable drop at the threshold value are recorded with the camera. Note that the threshold for levitation and that for the appearance of oscillations are very close to each other. A second method to determine the instability threshold is measurement of $Q_{c}$, obtained after drops have turned unstable. For a drop starting in the unstable state at $Q=Q_{t}$, the airflow is slowly reduced until a value is reached which results in a stable state: $Q=Q_{c}$. This second threshold $Q_{c}$ turns out to be slightly smaller than $Q_{t}$. However, the difference is comparable to the accuracy of the measurements of $Q_{c}$, so we cannot make any definite statements on whether or not the instability is hysteretic. In what follows we therefore plot the average threshold $Q_{m}$, obtained upon increasing the drop size and variation of the flow rate. $Q_{m}$ is determined as: $\left(Q_{t}+Q_{c}\right) / 2$. The error bar indicates the difference between the two measurement procedures.

After measurement of $Q_{c}$ the flow rate is further reduced, which finally results in a sessile drop state again. A snapshot is made at this zero flow rate (i.e., sessile drop; Fig. 6.5a), and the drop size $R$ is determined as the maximum radius of the sessile drop in top view. To reduce as much as possible the influence of any possible airflow fluctuations coming from e.g. variations in the substrate or hydrophobic grid fixation, all data points are measured at a fixed position on the substrate. To study the influence of viscosity on the drop dynamics, two liquids are used: water $(1 \mathrm{mPa} s)$ and a water-glycerine mixture (60 $\mathrm{mPa} \mathrm{s})$. The resulting dynamics are characterized by liquid viscosity $\eta_{l}$, drop size $R$, flow rate $Q$ and oscillation frequency $f$.

\subsection{Experimental results}

\subsubsection{Low-viscosity drops}

In this section we study the stability and dynamics of levitated water drops $\left(\eta_{l}=1 \mathrm{mPa} \mathrm{s}\right)$. This is reminiscent to the classical Leidenfrost drops, levitated above a hot substrate [22]. By varying the drop radius $R$ and airflow rate $Q$, the threshold for drop oscillations $\left(R_{t}, Q_{m}\right)$ is determined. Results for water are plotted in Fig. 6.4, as circles. The open circles are oscillations without detachment from the needle. In these cases, the size of the drop is measured in a sessile state. The solid circles correspond to violent oscillations or a chimney, which can lead to the detachment from the needle. The size is then approximated in the unstable levitated state. Clearly, the threshold drop size 
$R$ decreases with flow rate. The smallest drops investigated here are stable up to very high flow rate, while the largest drops destabilize even at very small $Q$. A chimney was, for example, observed for the smallest flow rate and largest drop size $R \simeq 9.6 \mathrm{~mm}$ (top blue solid circle in Fig. 6.4). This point is indeed close to the blue dashed line that indicates the onset of the chimney instability for water drops, as determined for thermal Leidenfrost drops by Biance et al. [22] ( $R_{c} \simeq 4.0 \ell_{c}$, where $\ell_{c}$ is the capillary length). Interestingly the chimney instability was predicted to occur even at vanishing flow rate [24]. However, constraints in the control of extreme small flow rates limited measurements in this range of parameters.

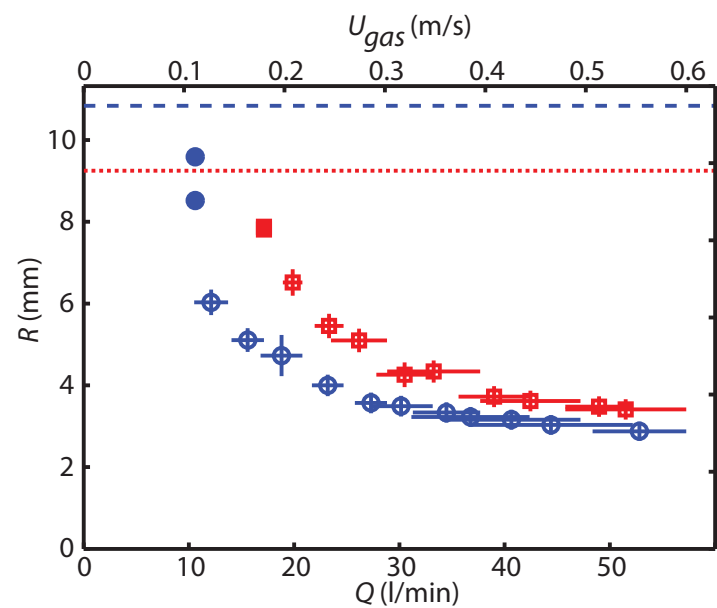

Figure 6.4: Measured instability threshold $Q_{m}$, for levitated drops. The upper axis gives the gas velocity, estimated by dividing the total flow rate by the area of the porous medium. Data represents all data points for water- and waterglycerine drops, in circles ( $\circ$ and $\bullet$ ) and squares ( $\square$ and $\square$ ), respectively. Since for the smallest flow rate the drop size could not be measured (it detaches from the needle), $R$ is measured in levitated state instead of sessile state. These points are therefore indicated by a solid symbol $(\bullet$ and $\mathbf{\square})$. Note that point corresponds to the chimney instability from Fig. 6.7b. The theoretical prediction of the critical radius for chimney instability is indicated by the blue dashed line and red dotted line for the used water and water-glycerine mixture, respectively. 
For all levitated drops, the oscillating motion is recorded at the threshold flow rate $Q_{t}$. Typical images obtained in the experiments are shown in Fig. 6.5. Fig. 6.5a is a sessile water drop, with $Q=0$, while snapshots (Fig. 6.5c-h) correspond to oscillating drops at non-zero flow rates. Once the water drops are unstable, the oscillations appear to be rather chaotic, i.e., a combination of modes (Fig. 6.5c). However, in a few cases as well one distinct mode was observed ranging from mode $n=2$ to $n=6$, as is shown in Fig. 6.5d-h.

In case of these well-defined modes, the oscillation frequency can be determined and compared to the prediction of Eq. (6.2). The results are shown in Fig. 6.6. For mode $n=3$, frequencies are measured for seven different drop sizes $R=3.2-6.1 \mathrm{~mm}$. Rescaling from Eq. (6.2) indeed collapses the data. Additionally the magnitude and trend are in quite good agreement with the inviscid theory (red solid line) for all modes.

\subsubsection{High-viscosity drops}

The viscosity of the drop is increased to investigate whether damping of the inner drop flow indeed suppresses star oscillations. Experiments shown in this section are carried out with liquid drops of a water-glycerine mixture $\left(\eta_{l}=60 \mathrm{mPa} s\right)$. Again the drop size $R$ and flow rate $Q$ are varied to determine the instability threshold for drop oscillations. The results are included in Fig. 6.4. The data points for large liquid viscosity are indicated with red squares $(\square, \square)$. For the solid red squared data points, a chimney instability is observed, for which an air bubble pierces the center of the drop. Such a chimney is shown in Fig. 6.7b. The size of the drop could therefore be determined only from a drop in levitated state.

Comparing the threshold of high-viscosity drops with water drops, we observe a clear increase of the threshold. However, the dependence on viscosity is relatively weak, given that the liquid viscosity was increased by a factor of about 60. By contrast, the dynamics are strongly affected by the liquid viscosity. While the oscillations of water drops at threshold is chaotic and non-axisymmetric, the viscous drops display only axisymmetric oscillations: we observe clear 'breathing' modes (symbol with error bars in Fig. 6.5b), for which the levitated drop remains circular in top view while oscillating. The large viscosity of the liquid drop apparently damps all higher mode oscillations, and the formation of star drops is completely suppressed. A more detailed picture illustrating this dynamics is shown in Fig. 6.7a. Consecutive snapshots (top row) all depict circular drops and a space-time diagram of the drop edge illustrates the radial oscillating motion. This regular dynamics make it rel- 

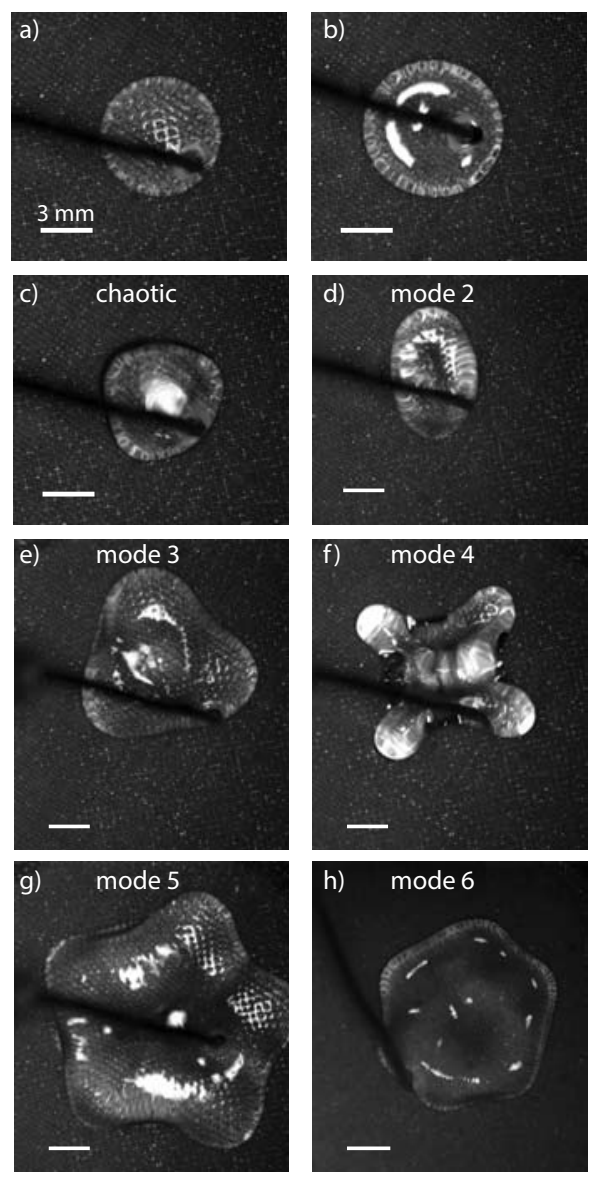

Figure 6.5: Examples of levitated drop instabilities. All images show water drops, except for (b) which is a water-glycerine drop. (a) Sessile water drop. (b) Levitating water-glycerine drop. (c) Chaotic mode water drop oscillation. (d) Water drop, mode $n=2(R=4.1 \mathrm{~mm}, f=13.8 \mathrm{~Hz})$. (e) Water drop, mode $n=3(R=6.1 \mathrm{~mm}, f=14.2 \mathrm{~Hz})$. (f) Water drop, mode $n=4(R=5.2$ $\mathrm{mm}, f=17.8 \mathrm{~Hz})$. (g) Water drop, mode $n=5(R=8.6 \mathrm{~mm}, f=14.3 \mathrm{~Hz})$. (h) Water drop, mode $n=6(R=6.1 \mathrm{~mm}, f=30.9 \mathrm{~Hz})$. 


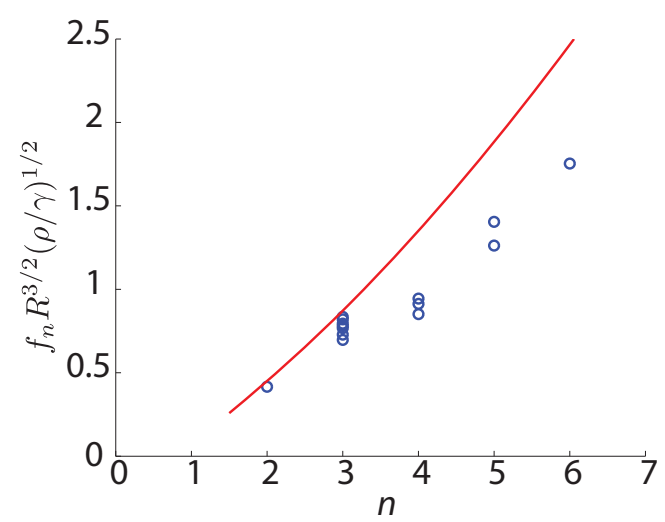

Figure 6.6: The frequency measured for faceted drops as shown in the images of Fig. 6.5. Each data point $\circ$, corresponds to one water drop measurement. The red solid line is the prediction from the corresponding eigen mode for a puddle, given by Eq. (6.2).

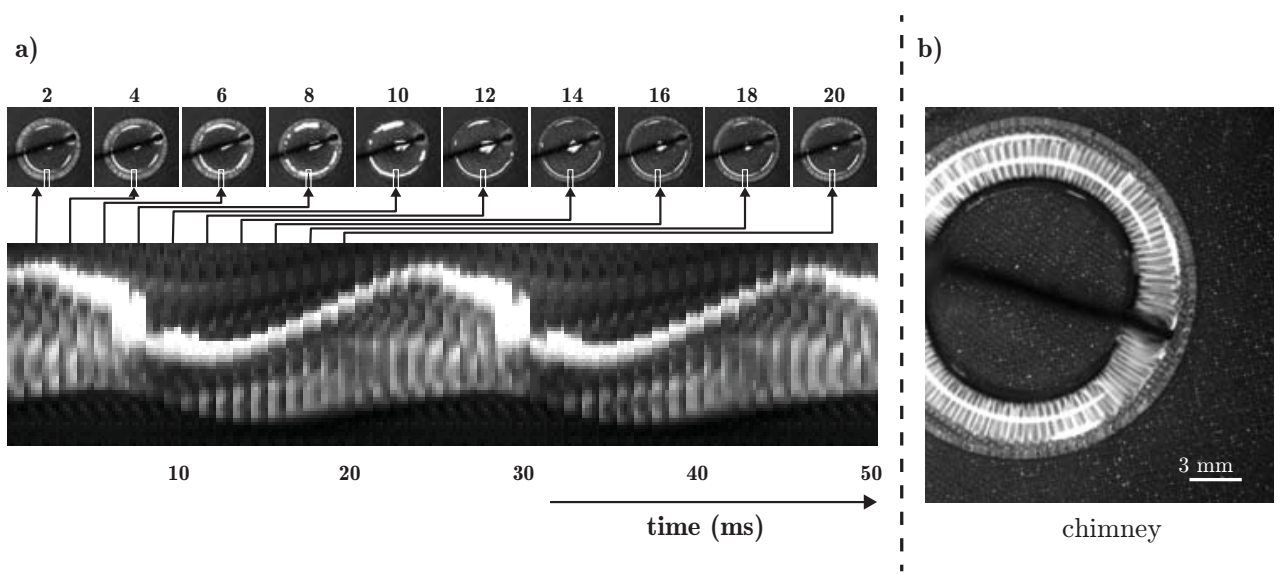

Figure 6.7: (a) Top row: an image sequence of the breathing mode oscillation of a large-viscosity drop (water glycerine, $60 \mathrm{mPa}$ s). As the oscillation amplitude is rather small, a space-time diagram is shown as well, which is built from slices similar to the white boxes indicated in the images. (b) For larger drop sizes we observe the formation of a chimney. 
atively easy to measure the main oscillation frequency for all data along the threshold curve (see Fig. 6.8). Note that in this measurement the frequency therefore is a function of $R\left(Q_{t}\right)$. Hence, a small radius in this figure automatically also means a relative large flow rate $Q_{t}$ and vice versa (see Fig. 6.4).

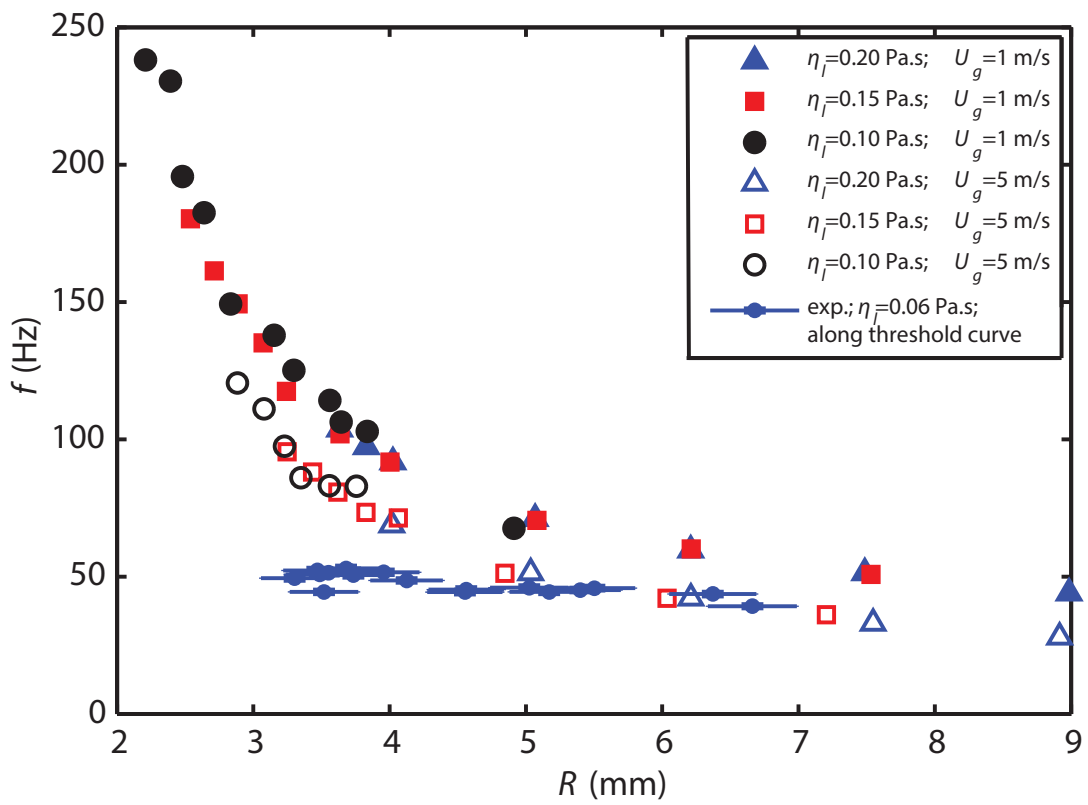

Figure 6.8: Measured oscillation frequency at threshold for high-viscosity drops (see Fig. 6.4) (blue dots with error bars), combined with numerical results. For the numerical simulations, the measured oscillation frequency (excitation frequency) as a function of the drop top view radius with airflow velocity 1 and $5 \mathrm{~m} / \mathrm{s}$, at three different liquid viscosities is shown. In the numerical simulations, frequency appears to be independent of liquid viscosity, decreases with increasing drop radius, and decreases with increasing airflow velocity.

Apart from this large contrast in shape deformations, also the measured oscillation frequencies are different from those measured with low-viscosity water drops. Frequencies for high viscosity drops are considerably higher, by a factor two or more, than the lowest mode $(n=2)$ of the inviscid Rayleigh and Lamb frequency for a drop of the same size, but compare rather well with numerical results for axisymmetric oscillations of an (inviscid) drop on 
an air cushion (see Secs. 6.4 and 6.5). One possible interpretation is that the gas flow and the liquid flow act as a coupled dynamic system that oscillates. In the case of water this oscillation, acting as a parametric forcing, directly leads to star oscillations which are well described by Eq. (6.1). However, viscosity affects or even suppresses star oscillations in high viscosity drops. As a result one essentially observes the frequency of this axisymmetric oscillation of the coupled system, which in contrast to that of the star oscillations only weakly depends on drop size. In summary, due to the suppression of star oscillations viscous drops reveal the underlying axisymmetric oscillation from which the stars originate. It is this axisymmetric oscillation that we will study numerically in the next sections.

Finally, we again observe chimneys when the drop size becomes too large, $R \approx 8 \mathrm{~mm}$ (see right panel of Fig. 6.7). Since the capillary length for the used water-glycerine mixture is, $\ell_{c} \sim 2.3 \mathrm{~mm}$, the chimney occurs at about $3.5 \ell_{c}$. This is consistent with earlier experiments on water drops [22] and theory [24] for which the critical radius $R_{c} \approx 4.0 \ell_{c}$ ( $R_{c}$ for the water-glycerine mixture is indicated by the red dotted line in Fig. 6.4).

\subsection{Numerical method}

We now investigate the dynamics of drops on an air cushion by numerical simulations. Since previous work, where drops were modeled by Stokes flow, did not result into any oscillation [24], inertia inside the drop must be important, and we now consider the opposite limit: potential flow. The latter is coupled to a viscous airflow, modeled in the lubrication approximation. The model is similar to that in Chapter 2 of this thesis, where it was used for simulating drop impact.

\subsubsection{Parameters \& dimensional analysis}

Similar to the experiments, the main parameters that will be varied are the drop volume $V$ and the gas flow, here denoted by the upward gas velocity $U_{g}$. Other parameters are the gas viscosity $\eta_{g}$ (lubrication approximation), liquid density $\rho_{l}$ (potential flow), and the surface tension $\gamma$. These can be combined into three dimensionless numbers. A measure for defining the drop size is the Bond number, Bo, taking into account gravity influence against surface tension influence: 


$$
\mathrm{Bo}=\sqrt{\frac{\rho_{l} R_{0}^{2} g}{\gamma}}=\frac{R_{0}}{\ell_{c}}
$$

where $R_{0}=\left(\frac{3 V}{4 \pi}\right)^{\frac{1}{3}}$ is the radius of the unperturbed spherical drop with volume $V$, and $g$ is the acceleration of gravity. $\ell_{c}$ is the capillary length, as defined in the Introduction. Secondly, we define the capillary number

$$
\mathrm{Ca}=\frac{\eta_{g} U_{g}}{\gamma},
$$

in which $U_{g}$ is a constant if we assume a uniform upward flow beneath the drop. Ca measures the influence of gas viscosity against surface tension and can be interpreted as the dimensionless gas velocity.

By setting a balance between the viscous forces of the gas flow and the square root of the inertial forces induced by the drop times the surface tension force, we finally introduce a dimensionless quantity which we will call the Ohnesorge number:

$$
\mathrm{Oh}=\frac{\eta_{g}}{\sqrt{\rho_{l} \gamma \ell_{c}}} .
$$

Note that this definition of Oh deviates from the standard definition, since it combines the viscosity of the gas and the density of the liquid.

Then, using $\ell_{c}, \frac{\gamma}{\eta_{g}}$, and $\frac{\gamma}{\ell_{c}}$ as the relevant length, velocity and pressure scales, the radial positions $r$, vertical positions $h$, velocities $u$, times $t$, and pressures $P$ are non-dimensionalized as, respectively

$$
\begin{aligned}
\widetilde{r} & =\frac{r}{\ell_{c}} ; \\
\widetilde{h} & =\frac{h}{\ell_{c}} ; \\
\widetilde{u} & =\frac{\eta_{g}}{\gamma} u ; \\
\widetilde{t} & =\frac{\gamma}{\ell_{c} \eta_{g}} t ; \\
\widetilde{P} & =\frac{\ell_{c}}{\eta_{g}} \frac{\eta_{g}}{\gamma} P=\frac{\ell_{c}}{\gamma} P .
\end{aligned}
$$

From now on we will drop the tildes and all variables will be dimensionless, unless stated otherwise. 


\subsubsection{Boundary Integral method coupled to lubricating gas layer}

The drop is assumed to consist of an incompressible and irrotational fluid, and can therefore be described by potential flow. The velocity field inside the drop is the gradient of a scalar velocity potential $\phi$. The Laplace equation,

$$
\nabla^{2} \phi=0,
$$

is valid throughout the whole drop including its surface contours. The Boundary Integral method is a way to solve this equation for $\phi$, with the proper boundary conditions [27-29]. For the levitated drop setup, the entire drop surface is a free surface, and the dynamic boundary condition for that surface is the unsteady Bernoulli equation:

$$
\frac{1}{\mathrm{Oh}^{2}}\left(\frac{\partial \phi}{\partial t}+\frac{1}{2}|\nabla \phi|^{2}\right)=-z-\kappa-P_{g},
$$

where $t$ is time, $z$ is the absolute height, and $\kappa$ is the local curvature at a point of the drop surface. The left-hand side describes the inertial effects of the drop, balanced by gravitational effects, the Young-Laplace pressure, and the influences by the airflow on the right-hand side. $P_{g}$ is the external pressure which is varying over the lower drop surface after introducing the gas flow. For this, the drop surface has been divided into two parts: the top of the drop where the surrounding pressure is atmospheric; and the bottom of the drop, where we deal with the lubrication pressure induced by the gas flow. The separation point between these two parts is taken at $r=R$, where $R$ is the topview radius, but results are unaffected by the precise location of the division $[23,24]$. The gas flow is mainly determined by the viscosity of the gas (Stokes flow). We assume that $R \gg h$. Note that the gas is defined to flow upwards from $z=0$ with uniform gas flow velocity $\mathrm{Ca}$, which will result in a predominantly radial gas flow below the drop with velocity $u(r, z)$. For deriving the axisymmetric lubrication approximation, we start with mass conservation of the incompressible gas flow

$$
\nabla \cdot \mathbf{u}=0 .
$$

Boundary conditions are

$$
\begin{aligned}
& \left.u_{z}\right|_{z=0}=\mathrm{Ca} \\
& \left.u_{z}\right|_{z=h}=\dot{h},
\end{aligned}
$$


where $\dot{h}$ is the vertical velocity of the drop surface. Furthermore, at the free fluid-air-interface, $z(r)=h(r)$, there is a kinematic boundary condition

$$
\frac{\partial h}{\partial t}=\left.u_{z}\right|_{z=h}-\left.\frac{\partial h}{\partial r} u_{r}\right|_{z=h},
$$

which is the unsteady part of the problem setting. Integrating the continuity equation (6.8) along $z$ (between 0 and $h$ ), applying Leibniz integral rule, substituting the boundary conditions, defining the average (radial) flow velocity $\bar{u}=\frac{1}{h} \int_{0}^{h} u_{r} d z$, and multiplying the equation with $r$ gives [24]

$$
\frac{\partial}{\partial r}(r h \bar{u})+r \dot{h}=r \text { Ca. }
$$

Applying the Stokes equation for this axisymmetric lubrication flow with zero velocity boundary conditions at $z=0$ and $z=h$ gives

$$
u=6 \bar{u}\left(\frac{z}{h}-\frac{z^{2}}{h^{2}}\right) \Rightarrow \frac{\partial P_{g}}{\partial r}=-\frac{12 \bar{u}}{h^{2}},
$$

in which $P_{g}$ is the pressure in the gas layer. Combining (6.10) and (6.9), and performing one integration leads to

$$
\frac{\partial P_{g}}{\partial r}=\frac{12}{r h^{3}}\left(-\frac{\Gamma}{2 \pi}+\int_{0}^{r} \hat{r} \dot{h} d \hat{r}\right)
$$

where

$$
\Gamma=2 \pi \int_{0}^{r} \hat{r} \mathrm{Ca} d \hat{r}=\pi \mathrm{Car}^{2} .
$$

is the radius-dependent volume-airflux. The first term on the right-hand-side of (6.11) is the gas flow term; the second term concerns the motion of the drop interface. $\Gamma$ is radially increasing, since the gas is accumulating beneath the drop.

\subsection{3 'Artificial' viscous damping}

Since viscous effects inside the drop are neglected, all motions (waves, oscillations, vertical translations, ...) will be undamped, as long as we do not apply any form of damping. Indeed, simulations with realistic input parameters (radius and airflow velocity) lead to a quick blow-up of surface wave amplitudes or the drop receiving a pressure pulse from below (when $h$ becomes too small at some point). In particular, we were unable to produce any steady solutions 
without the implementation of damping. We therefore need to introduce a damping term in Eq. (6.7). We opted to follow a physically motivated way using 'viscous potential flow' (VPF) [30]. Applying VPF to a free surface generally leads to an additional term in the unsteady Bernoulli equation valid on this surface, operating as pure damping term. The additional term is the local normal stress, $2 \eta_{l} \frac{\partial^{2} \phi}{\partial n^{2}}[31], \eta_{l}$ being the liquid viscosity, such that (6.7) transforms into:

$$
\frac{1}{\mathrm{Oh}^{2}}\left(\frac{\partial \phi}{\partial t}+\frac{1}{2}|\nabla \phi|^{2}\right)=-z-\kappa-P_{g}+2 \Lambda \frac{\partial^{2} \phi}{\partial n^{2}},
$$

where

$$
\Lambda=\frac{\eta_{l}}{\eta_{g}}
$$

We have to make some remarks on this 'artificial' damping method. First, it is unclear to what extent the model represents a true viscous drop, since viscosity in general induces vorticity in the flow, which, of course, is absent in the simulation. It turned out that the liquid viscosity required to obtain stable numerical solutions is quite large, about 100 times the viscosity of water. Consequently, we will treat $\Lambda$ as a numerical damping constant, rather than a physical viscous effect of the liquid. Secondly, for too large damping, this method amplifies numerical deviations in the code: the normal stress term contains numerical approximations to derivatives, which are now multiplied by a large factor. Summarizing, both requirements together set a narrow window for our liquid viscosity:

$$
0.10 \mathrm{~Pa} \cdot \mathrm{s} \leq \eta_{l} \leq 0.30 \mathrm{~Pa} \cdot \mathrm{s} .
$$

Outside this range we were unable to generate reliable and stable numerical results.

\subsubsection{Numerical details}

In the numerical process, the Laplace equation is solved every time step, similar to Bergmann et al., 2009 [29]. The size of a time step varies over the simulation, and depends on the instantaneous drop dynamics. The time step is small enough to prevent neighboring nodes from crossing each other. For a steady drop, or a falling drop, the time step may be of order 0.001 time units (typically of order $1 \cdot 10^{-2} \mathrm{~ms}$ ), while an oscillatory scenario, with strong 
curvatures and large nodal velocities, could end up with time steps of order $1 \cdot 10^{-5} \mathrm{~ms}$.

In general, the simulation is initiated by a spherical drop falling from small starting height in the order of 0.10 capillary length. However, close to the chimney instability (see Sec. 6.5.1), it is necessary to start with a more 'gentle' initial shape (i.e., closer to the expected 'Leidenfrost' shape for these kind of drop sizes), such that the drop does not get unstable due to the impact of the drop after the free fall.

The drop contour is characterized by $r$ and $z$ for $r>0$. For the initial spherical drop (in the first time steps of the simulation), this surface line consists of about 60 nodes, depending on the size of the drop (a smaller drop results in a smaller number of nodes). The number of nodes will vary during the simulation, set by the (maximum) local curvatures on the line and the closeness to the symmetry axis $r=0$; the largest node density is set around the bottom and top center of the drop. It has been checked that further increasing the number of nodes does not change the results significantly.

\subsection{Numerical results}

To easily compare with experiments, the figures in this section are in SI units.

\subsubsection{Steady shapes \& chimneys}

The numerical scheme described above can indeed lead to steady levitated drops, chimneys, or oscillatory states, depending on the model parameters. Here we first focus on steady shapes, an example of which is shown in Fig. 6.9. For two different initial conditions (top left panel), the drop relaxes to the same final shape (bottom right panel). In all cases, the drop shape depends only on Bo and $\mathrm{Ca}$, and is independent of $\mathrm{Oh}$ and $\Lambda$.

The pressure profile at the bottom of the drop has a similar shape for every drop size and airflow velocity, from the moment the steady shape has been reached. An example is shown in Fig. 6.10. The largest pressure is at $r=0$, and it decreases to atmospheric pressure for $r \rightarrow R$. The pressure gradient is largest at the neck radius, $r=r_{n}$, such that the pressure profile resembles a plateau. The minimal gap height in this example is of the order of $100 \mu \mathrm{m}$.

Figure 6.11 shows an example of a chimney instability. The respective volumes of the red and blue curves differ by a small amount. Yet, the bigger 

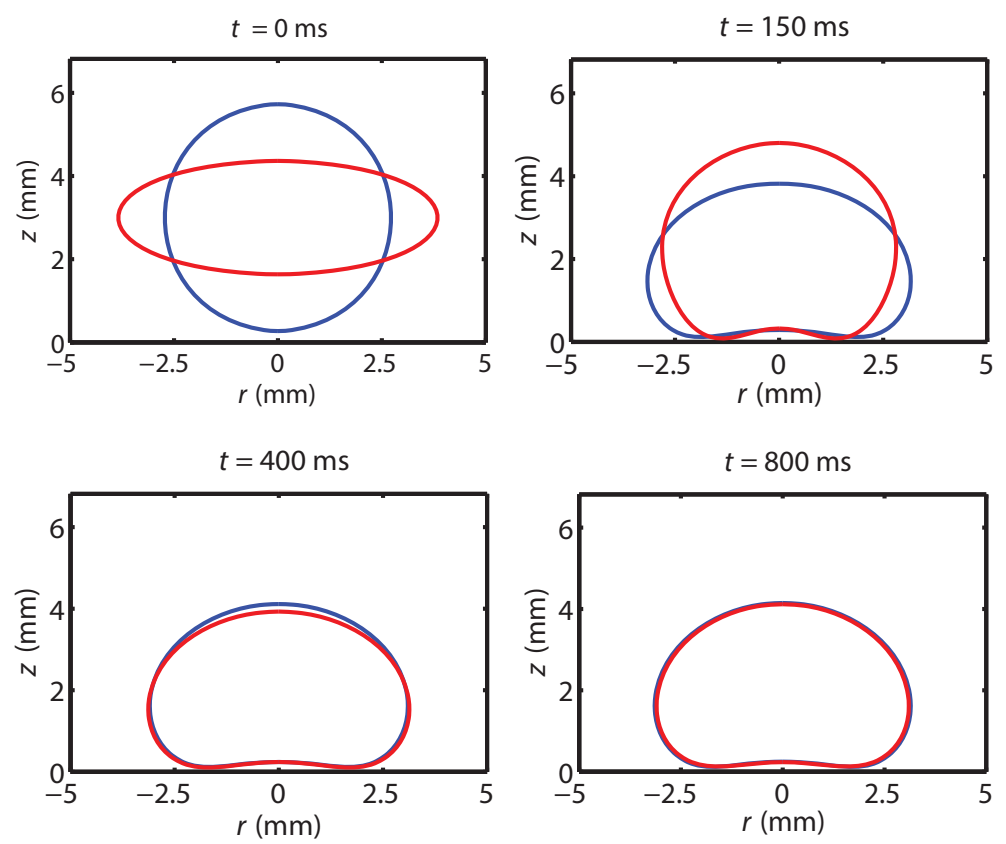

Figure 6.9: Two different initial drop shapes (one spherical, one elliptical) of equal volume, converging to the same steady end shape. $\mathrm{Bo}=1, \mathrm{Ca}=2.5 \cdot 10^{-4}$, $\Lambda=11 \cdot 10^{3}$. See Fig. 6.10 for the corresponding pressure profile.

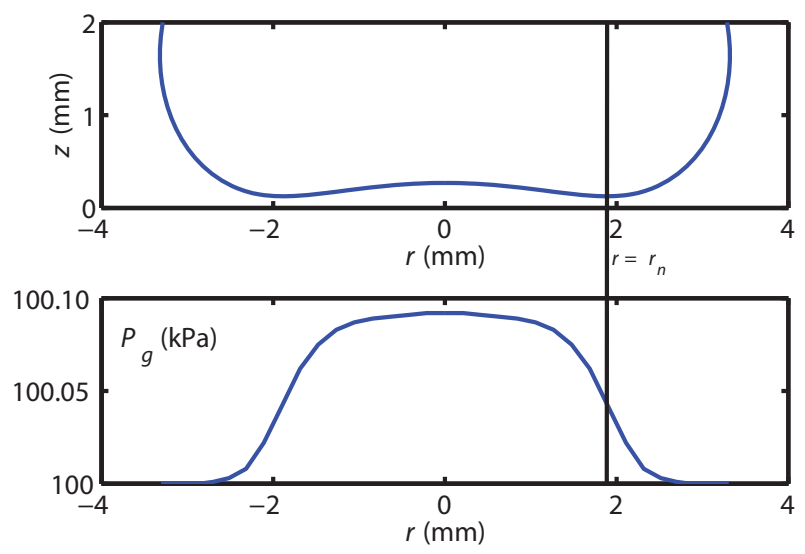

Figure 6.10: Pressure profile $\left(P_{g}\right)$ at the bottom of a steady levitated drop. The largest pressure gradient is typically at the neck, $r=r_{n}$, such that the profile resembles a plateau. $\mathrm{Bo}=1, \mathrm{Ca}=2.5 \cdot 10^{-4}, \Lambda=11 \cdot 10^{3}$ 

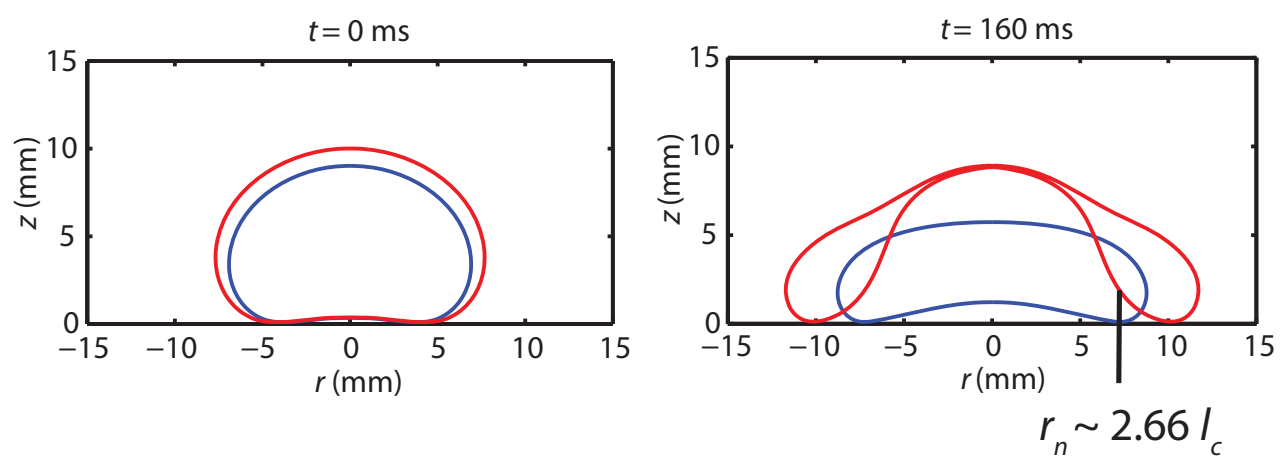

Figure 6.11: Chimney instability. Shown is the evolution of two drops, with almost equal volumes. The largest drop apparently has a radius just above the chimney threshold, which appears to be about $2.7 \ell_{c}$, or $7.3 \mathrm{~mm}$, while the smallest has a radius just below. The large drop does not remain stable, due to the gas pocket breaking through; the small drop keeps its steady shape. $\mathrm{Bo}=2.25$ and $2.5, \mathrm{Ca}=2.5 \cdot 10^{-5}, \Lambda=11 \cdot 10^{3}$.

drop develops a chimney instability, while the smaller one exhibits a steady state. The limit of drop size for the chimney instability agrees with expectations from Snoeijer et al., 2009 [24]. We deduce from Fig. 6.11 a threshold neck radius of about $2.7 \ell_{c}$ for a gas flow velocity of $0.1 \mathrm{~m} / \mathrm{s}$. The dimensionless airflux $\chi$ which is introduced in Snoeijer et al., 2009 [24] is in our case $\chi=\frac{6 \Gamma\left(r_{n}\right)}{\pi r_{n}}=\frac{6 \pi \cdot \mathrm{Ca} \cdot r_{n}^{2}}{\pi r_{n}} \sim \frac{6 \pi 0.1\left(2.7 \ell_{c}\right)^{2}}{\pi\left(2.7 \ell_{c}\right)}=4.42 \cdot 10^{-3}$. Extrapolation in Fig. 12 of Snoeijer et al., 2009 [24] shows that this $2.7 \ell_{c}$ agrees with the theoretical prediction coming from the lubrication approximation. The threshold for chimneys is at smaller drop size than the experimentally observed threshold (Fig. 6.4), which can be explained by the smaller incoming airflow velocity in the experiments, compared to numerical simulations. According to Snoeijer et al., 2009 [24], for increasing $\chi$, the threshold for chimneys is at smaller drop size, and $\chi$ in the numerical simulations is indeed large with respect to $\chi$ in the experiments.

\subsubsection{Drop oscillations}

\section{Observations}

The second scenario of interest we studied is drop instability leading to oscillations. An example is shown in Fig. 6.12, showing the drop contours during 

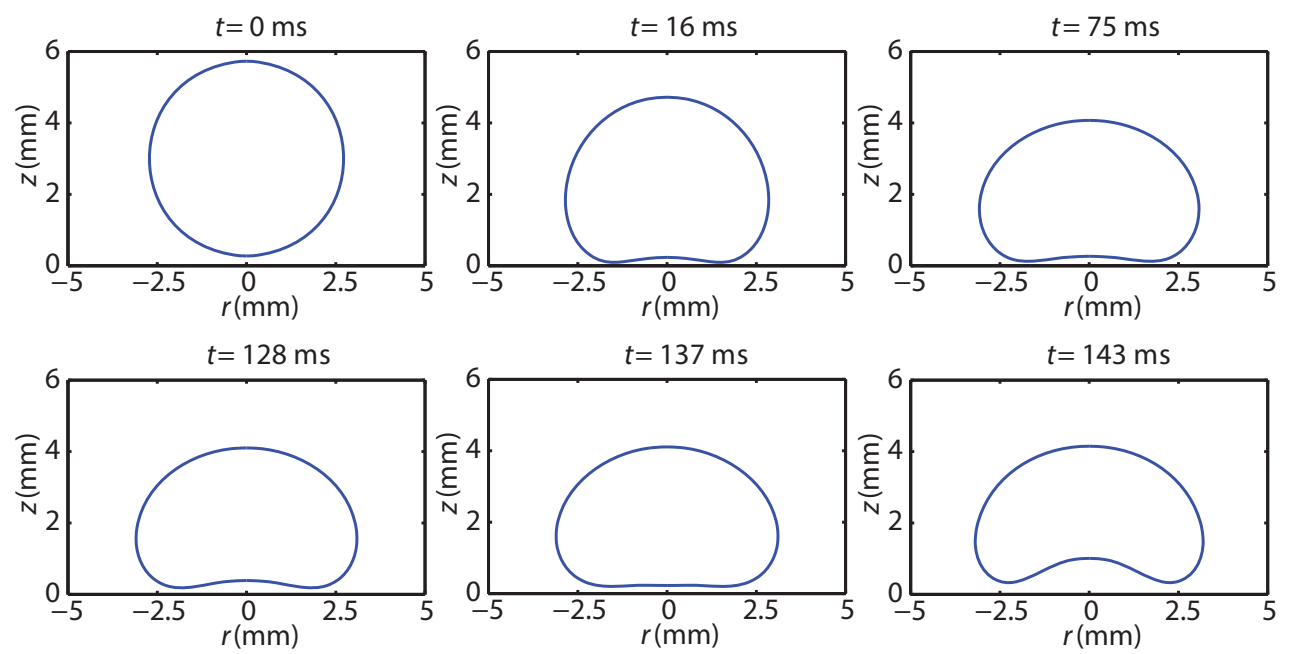

Figure 6.12: Time sequence from the evolution of the oscillatory instability of a levitated drop. The simulation is initiated by a spherical drop, released from small height $(0.27 \mathrm{~mm})$ (top-left). The top panel row shows the process from the spherical drop shape to an intermediate steady shape. The bottom panel row shows the oscillatory behavior of the drop at a later point in time. $\mathrm{Bo}=1, \mathrm{Ca}=2.5 \cdot 10^{-4}, \Lambda=8.2 \cdot 10^{3}$. 
the evolution of the oscillations for an unstable scenario. The first three panels (top row) show the process of the drop converging towards the 'Leidenfrost' shape. It takes about $75 \mathrm{~ms}$ for the drop to adopt a nearly steady shape (topright), but in the next phase surface oscillations with increasing magnitude are visible (bottom sequence). The drop oscillates in both radial and vertical direction. The two states between which the drop 'bounces' are clearly visualized in the last two frames of Fig. 6.12, and in Fig. 6.13, supplemented with velocity profiles. The velocity profiles show that the liquid velocity, and therefore the oscillations and momentary liquid flows are mainly in the vertical direction. Air is released from the gas-pocket at the bottom of the drop around one of the extremes and is gathered again towards the other: the system 'breathes'.
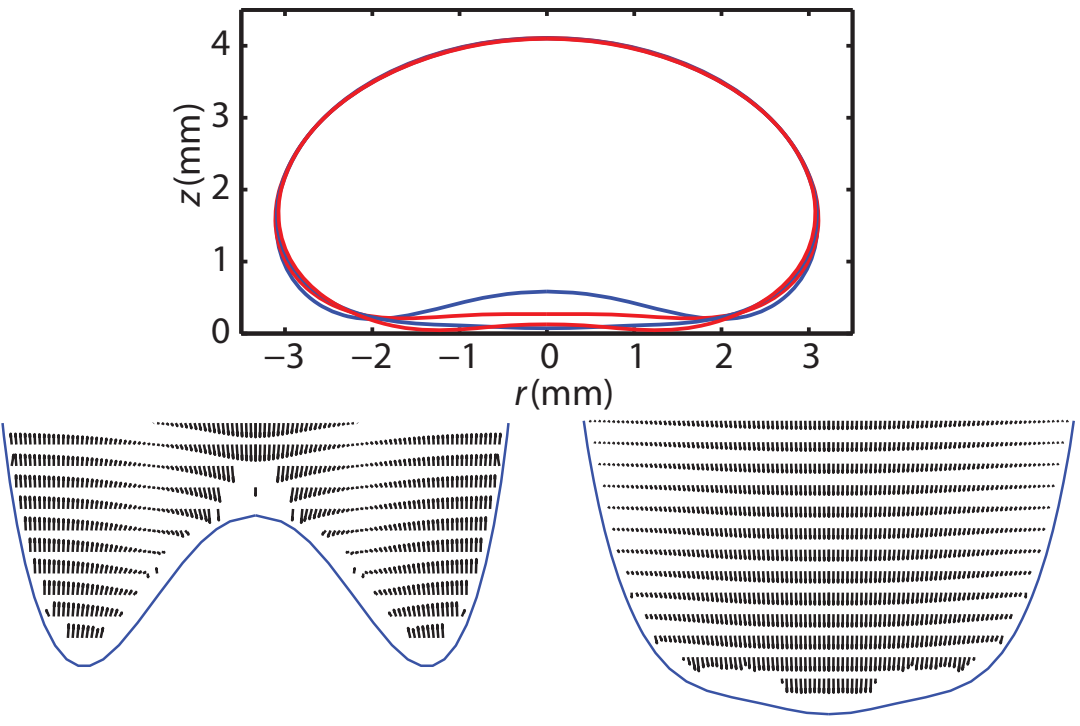

Figure 6.13: Drop contours during the final stages of the simulation in an unstable scenario (see Fig. 6.12). Blue contours are the two extremes, red lines are intermediate. The bottom two plots show the velocity profile inside the drop for the extremes. Note that the liquid velocities, and therefore the oscillations as well, are mainly in the vertical direction. $\mathrm{Bo}=1, \mathrm{Ca}=2.5 \cdot 10^{-4}$, $\Lambda=8.2 \cdot 10^{3}$.

Similarly to experiments, there exists a drop size threshold and a gas flux threshold above which the surface oscillations appear. In Fig. 6.14a, no drop oscillations are visible. In Fig. 6.14 we plot the time dynamics $R(t)$ for different parameters. In Fig. 6.14b, the oscillation amplitude visibly saturates at 

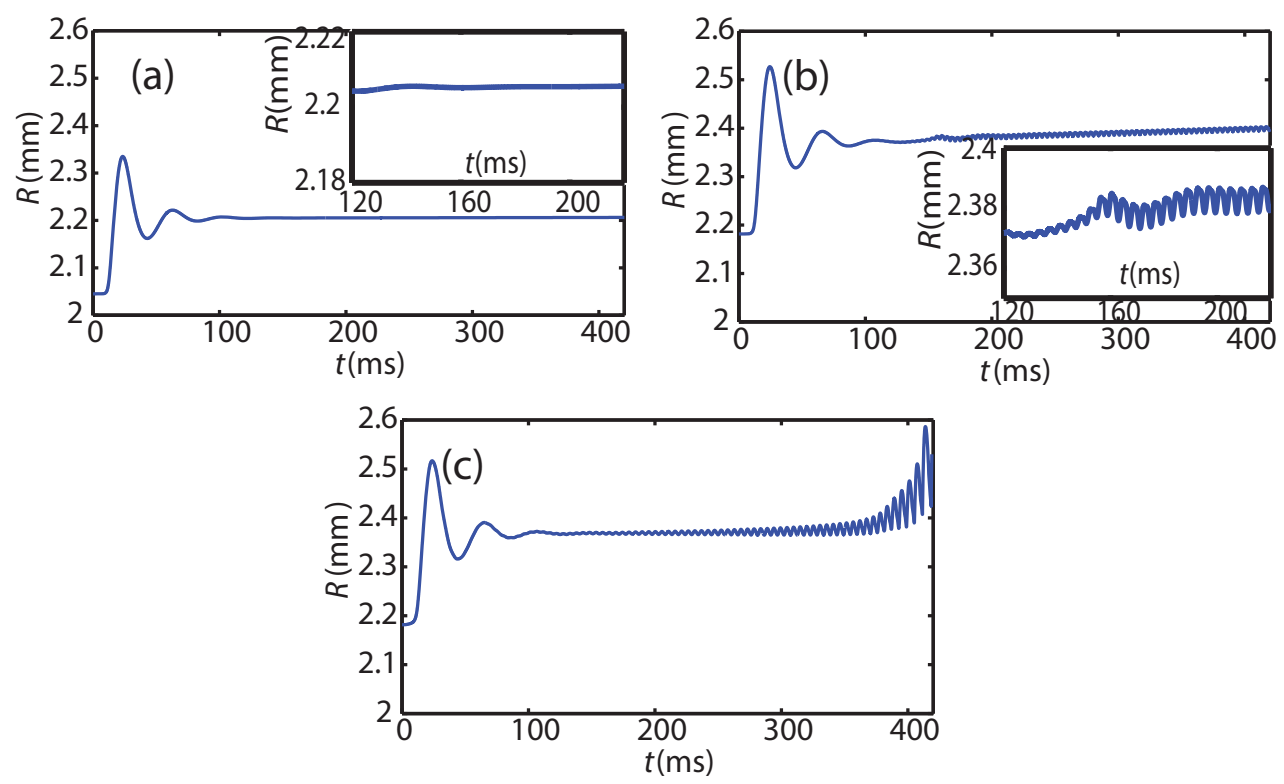

Figure 6.14: Top view radius as a function of time for (a) a stable drop, (b) a case around the transition, and (c) an unstable drop. In the first part of each plot (up to about $100 \mathrm{~ms}$ ) the initial, spherical shape of the drop stabilizes towards the 'Leidenfrost' state. After this stabilization the oscillations become visible which typically have a much larger frequency (see insets). (a) $\mathrm{Bo}=0.75$, $\mathrm{Ca}=2.5 \cdot 10^{-5}$; (b) $\mathrm{Bo}=0.80, \mathrm{Ca}=5 \cdot 10^{-5}$; (c) $\mathrm{Bo}=0.80, \mathrm{Ca}=5 \cdot 10^{-4} . \Lambda=5.5 \cdot 10^{3}$ in all three cases. 
some small level. The threshold for oscillations is determined for the smallest asymptotically detectable oscillation. In Fig. 6.14c, the oscillation amplitude starts to grow after some time and the drop does not reach any asymptotic state, which is clearly an unstable situation. This explosive scenario is observed at some distance beyond the oscillatory threshold. The growth rate of the instability depends on the gas flux and the drop size, but especially on the damping coefficient $\Lambda$.

\section{Stability diagram}

We investigated the threshold for obtaining surface oscillations by varying the drop size and the gas flow velocity for $\eta_{l}=0.20 \mathrm{~Pa} \cdot \mathrm{s}$, resulting in the stability diagram shown in Fig. 6.15. We observe a decreasing transition line, similar to the experimental results in Fig. 6.4 with larger drops becoming unstable at smaller airflow velocity. An important observation is that the threshold is at much larger values (approximately a factor of 10 larger) for the ascending airflow velocity (factor of about 10), compared to the experiments (see Fig. 6.4). The relative shape of the transition line is similar in all numerical stability diagrams obtained for different $\eta_{l}$ and $\rho_{l}$, but for decreasing damping factor and/or increasing liquid density, the line moves in both the left and the downward direction. In experiments, the influence of the liquid viscosity on the threshold of the instability turned out to be very small. Obviously, our artificial implementation of damping is a plausible reason for the discrepancy between experiments and numerical simulations concerning the threshold.

\section{Frequency analysis}

In Fig. 6.8, we show the measured drop oscillation frequencies from the simulations against the drop radius, for different $\eta_{l}$ and $U_{g}$, and compare them to the experimental values for a water-glycerine drop. The oscillation frequencies decrease with increasing drop size, and decrease slightly with increasing gas flow velocity. The observed frequencies appear to be independent of the damping factor.

The frequencies extracted from numerical simulations are compared to those measured experimentally on axisymmetric oscillations for highly viscous drops: the agreement is good for the large radii ( $R$ from 5 to $7 \mathrm{~mm}$ ), but there are some discrepancies for smaller drop radius. To understand this overestimation from the simulations, it should be pointed out that the magnitude of oscillations can be much larger in experiments than in the simulations. Non- 


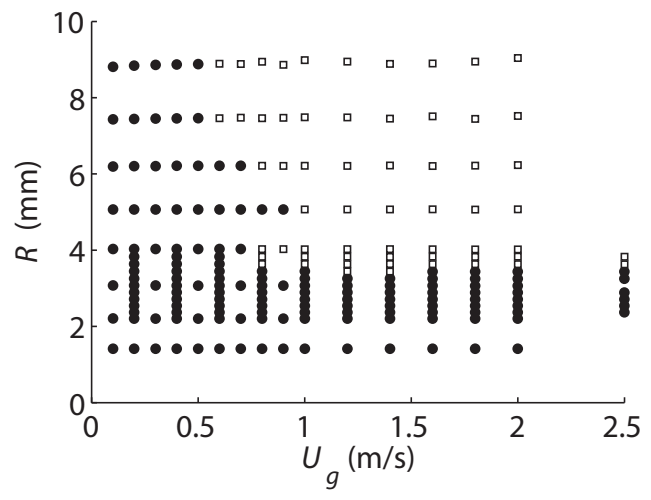

Figure 6.15: Top view radius $R$ versus airflow velocity $U_{g}$ stability diagram for $\Lambda=11 \cdot 10^{3}$. Black dots point out stable configurations: the drop has no tendency to oscillate; white squares indicate oscillating drops.

linear effects at finite amplitude generally lead to a decrease of the response frequency of drops [32], which is especially prevalent for small drops.

\subsection{Discussion}

In this chapter we investigated the dynamics of drops levitated by a gas cushion with constant and uniform influx. Various dynamics are observed, in both experiments and numerical simulations: drops either exhibit stable shapes, oscillate, or, undergo a 'chimney' instability in which the gas pocket breaks through the center of the drop.

Our experimental results show that for both high-viscosity and low-viscosity drops, the threshold flow rate for oscillatory instability continuously increases when decreasing the drop size. At very low $Q$, we do not reach the oscillatory state, since there is a maximum drop size beyond which the chimney instability sets in, as predicted by Snoeijer et al. [24]. The trends are very similar for both viscosities, but the threshold is slightly higher at high viscosity. This dependence on viscosity is relatively weak in our experiments; whereas the viscosity was increased by a factor 60 , the threshold flow rate only increased by less than 50\%. By contrast, the drop dynamics are strongly influenced by viscosity. Non-axisymmetric modes and chaotic oscillations could be observed near the threshold in oscillating water drops, while in the high viscosity case, only the 'breathing' mode is observed. From this observation 
we infer that axisymmetric modes rather than the breaking of the azimuthal symmetry constitute the origin of the spontaneous appearance of oscillations.

All these features have been reproduced numerically, by coupling inviscid Boundary Integral code for the drop to a viscous lubrication model for the gas flow. Because potential flow without any damping was unstable in the interesting time range for the evolution of drop oscillations, an artificial damping needed to be introduced, which enabled the observation of both stable drop shapes and oscillations. The idea of a coupling between potential flow liquid and Stokes gas flow proved to be very useful to study the equilibrium shapes of Leidenfrost drops and deforming dynamics of these drops, or (the dimple formation of) impacting drops at room temperature [25] and impacting evaporating drops. Interestingly, for the impacting drop simulations, no damping needed to be involved (because the time range in which we are interested was much shorter).

In the numerical simulations of Leidenfrost drops it is observed that, within a certain range of the parameter space, initially stable (steady) drop shapes gradually start to oscillate. Frequencies of the oscillations are in reasonable agreement with experimental results, especially for large drops. The most important difference between our numerical simulations and the experiments is that the threshold strongly depends on the amount of damping (the larger the numerical damping constant $\Lambda$, the larger the threshold drop radii and flow velocities). Furthermore, the threshold velocity lies an order of magnitude away from the experimental one, for simulations with $\Lambda=11 \cdot 10^{3}$. The minimal value of $\Lambda$ is about $5.5 \cdot 10^{3}$, at which almost all drop sizes and flow velocities led to unstable scenarios. Therefore, a more realistic way of damping needs to be implemented to investigate the position of the threshold.

In both experiments and simulations, the air is injected from below. This is different from Leidenfrost drops, which float on their own vapor, but their dynamics are very similar. Hence, it is verified that the phenomenon of star oscillations does not require any thermal driving, contrarily to previous suggestions [21]. This confirms the preliminary experimental observation [12] that the origin of drop oscillations are purely governed by fluid dynamics. The picture that emerges is that the oscillations appear due to an instability of the coupled system of the lubricating gas flow and the deformable drop. In the experiments, once the oscillations appear, 'stars' naturally develop as a parametric instability for low-viscosity drops, in a way similar to water drops placed on an oscillating plate [19]. At higher viscosity, the star formation is suppressed by viscous damping and only axisymmetric modes appear. This is 
similar for the onset of Faraday waves, induced by periodic forcing of a horizontal free surface [33]. Indeed, a large viscosity suppresses the appearance of the parametric instability that leads to Faraday waves. Therefore, this confirms that faceted star shapes are a result of parametric excitation that can only appear at sufficiently small damping (i.e., liquid viscosity).

Though the exact mechanism that leads to oscillations remains to be explained, our study unveiled interesting clues to understand the phenomenon and could dismiss other mechanisms. Interestingly, the Reynolds number for the high viscosity drops in experiments is relatively small $\operatorname{Re}_{l} \sim \widetilde{U}_{l} R \rho_{l} / \eta_{l} \sim$ $0.1 R f R \rho_{l} / \eta_{l} \approx 1$ (where we estimate the oscillation amplitude as $10 \%$ of $R$ ) and still spontaneous oscillations are observed above a threshold radius and gas flow rate. Previous numerical simulations based on Stokes flow for both the drop and the gas displayed no oscillations [24]. This raises the question of whether oscillations indeed cease to exist when further reducing the Reynolds number, i.e., by increasing the liquid viscosity. It will be a challenge to investigate this regime experimentally due to practical difficulties of working with such a highly viscous liquid. Other valuable information could also be provided by flow visualization inside the drop and the gas, since the results suggest a crucial coupling between the drop flow and the gas flow. The latter method applies not only to the experiments, but also to the simulations.

\section{References}

[1] J. G. Leidenfrost, De Aquae Communis Nonnullis Qualitatibus Tractatus, Duisburg on Rhine, Germany (1756).

[2] D. Quéré, "Leidenfrost dynamics", Annu. Rev. Fluid Mech. 45, 197-215 (2013).

[3] J. C. Burton, A. L. Sharpe, R. C. A. van der Veen, A. Franco, and S. R. Nagel, "The geometry of the vapor layer under a Leidenfrost drop", Phys. Rev. Lett. 109, 074301 (2012).

[4] H. Linke, B. J. Alemán, L. D. Melling, M. J. Taormina, M. J. Francis, C. C. Dow-Hygelund, V. Narayanan, R. P. Taylor, and A. Stout, "Self-propelled Leidenfrost droplets", Phys. Rev. Lett. 96, 154502 (2006).

[5] G. Lagubeau, M. Le Merrer, C. Clanet, and D. Quéré, "Leidenfrost on a ratchet", Nat. Phys. 7, 395-398 (2011). 
[6] A. Wurger, "Leidenfrost Gas Ratchets Driven by Thermal Creep", Phys. Rev. Lett. 107, 164502 (2011).

[7] K. Adachi and R. Takaki, "Vibration of a flattened drop. I. Observation", J. Phys. Soc. Jap. 53, 4184-4191 (1984).

[8] R. Takaki and K. Adachi, "Vibration of a flattened drop. II. Normal Mode Analysis" J. Phys. Soc. Jap. 54, 2462-2469 (1985).

[9] N. J. Holter and W. R. Glasscock, "Vibrations of evaporating liquid drops", J. Acous. Soc. Am. 24, 682-686 (1952).

[10] D. E. Strier, A. A. Duarte, H. Ferrari, and G. B. Mindlin, "Nitrogen stars: morphogenesis of a liquid drop", Physica A 283, 261-266 (2000).

[11] A. Snezhko, E. Ben Jacob, and I. S. Aranson, "Pulsating-gliding transition in the dynamics of levitating liquid nitrogen droplets", New J. Phys. 10, 043034 (2008).

[12] P. Brunet and J. H. Snoeijer, "Star drops formed by periodic excitation and on an air cushion A short review", Eur. Phys. J. Spec. Top. 192, 207-226 (2011).

[13] X. Noblin, A. Buguin, and F. Brochard-Wyart, "Triplon modes of puddles", Phys. Rev. Lett 94, 166102 (2005).

[14] M. Okada and M. Okada, "Observation of the shape of a water drop on an oscillating Teflon plate", Exp. Fluids 41, 789-802 (2006).

[15] C. L. Shen, W. J. Xie, and B. Wei, "Parametrically excited sectorial oscillation of liquid drops floating in ultrasound", Phys. Rev. E 81, 046305 (2010).

[16] Y. Fautrelle, J. Etay, and S. Daugan, "Free-surface horizontal waves generated by low-frequency alternating magnetic fields", J. Fluid Mech. 527, 285-301 (2005).

[17] M. Papoular and C. Parayre, "Gaz-film levitated liquids: shape fluctuations of viscous drops", Phys. Rev. Lett. 78, 2120-2123 (1997).

[18] M. Perez, Y. Brechet, L. Salvo, M. Papoular, and M. Suery, "Oscillation of liquid drops under gravity: Influence of shape on the resonance frequency", Europhys. Lett. 47, 189-195 (1999). 
[19] N. Yoshiyasu, K. Matsuda, and R. Takaki, "Self-induced vibration of a water drop placed on an oscillating plate", J. Phys. Soc. Jap. 65, 2068-2071 (1996).

[20] L. Rayleigh, "On the capillary phenomena of jets", Proc. R. Soc. 29, 71-97 (1879).

[21] N. Tokugawa and R. Takaki, "Mechanism of self-induced vibration of a liquid drop based on the surface tension fluctuation", J. Phys. Soc. Jap. 63, 1758-1768 (1994).

[22] A.-L. Biance, C. Clanet, and D. Quéré, "Leidenfrost drops", Phys. Fluids 15, 1632-1637 (2003).

[23] L. Duchemin, J. R. Lister, and U. Lange, "Static shapes of levitated viscous drops", J. Fluid Mech. 533, 161-170 (2005).

[24] J. H. Snoeijer, P. Brunet, and J. Eggers, "Maximum size of drops levitated by a air cushion", Phys. Rev. E 79, 036307 (2009).

[25] W. Bouwhuis, R. C. A. van der Veen, T. Tran, D. L. Keij, K. G. Winkels, I. R. Peters, D. van der Meer, C. Sun, J. H. Snoeijer, and D. Lohse, "Maximal air bubble entrainment at liquid-drop impact", Phys. Rev. Lett. 109, 264501 (2012),

See Chapter 2 of this thesis.

[26] I. A. Larmour, S. E. J. Bell, and G. C. Saunders, "Remarkably simple fabrication of super hydrophobic surfaces using electroless galvanic deposition", Angewandte Chemie Int. Ed. 46, 1710-1712 (2007).

[27] C. Pozrikidis, Introduction to theoretical and computational fluid dynamics, Oxford University Press (1997).

[28] H. N. Oguz and A. Prosperetti, "Dynamics of bubble growth and detachment from a needle", J. Fluid Mech. 257, 111-145 (1993).

[29] R. P. H. M. Bergmann, D. van der Meer, S. Gekle, J. van der Bos, and D. Lohse, "Controlled impact of a disk on a water surface: cavity dynamics", J. Fluid Mech. 633, 381-409 (2009).

[30] D. D. Joseph, "Viscous potential flow", J. Fluid Mech. 479, 191-197 (2003). 
[31] J. M. Gordillo, "Axisymmetric bubble collapse in a quiescent liquid pool. I.: theory and numerical simulations", Phys. Fluids 20, 112103 (2008).

[32] W. R. Smith, "Modulation equations for strongly nonlinear oscillations of an incompressible viscous drop", J. Fluid Mech. 654, 141-159 (2010).

[33] K. Kumar and L. S. Tuckerman, "Parametric instability of the interface between two fluids", J. Fluid Mech. 279, 49-68 (1994). 


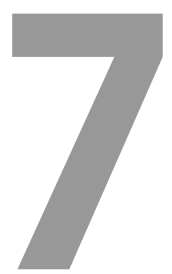

\section{Drop shaping by laser-pulse impact ${ }^{* \dagger}$}

We show how the deposition of laser energy induces propulsion and strong deformation of an absorbing liquid body. Combining high-speed with stroboscopic imaging we observe that a millimeter-sized dyed water drop hit by a milli-Joule nanosecond laser-pulse propels forward at several meters per second and deforms until it eventually fragments. The drop motion results from the recoil momentum imparted at the drop surface by water vaporization. We measure the propulsion speed and the time-deformation law of the drop, complemented by boundary integral simulations. The drop propulsion and shaping are explained in terms of the laser-pulse energy, the drop size, and the liquid properties. These findings are, for instance, crucial for the generation of extreme ultraviolet (EUV) light in nanolithography machines.

*Published as: A.L. Klein, W. Bouwhuis, C.W. Visser, H. Lhuissier, C. Sun, J.H. Snoeijer, E. Villermaux, D. Lohse, H. Gelderblom, "Drop shaping by laser-pulse impact", Phys. Rev. Appl. 3, 044018 (2015).

${ }^{\dagger}$ The numerical simulations in this chapter are part of the present thesis. The experimental work is due to Alexander Klein. 


\section{$7.1 \quad$ Introduction}

Laser-induced phase change in liquids can lead to a violent response: deformation and disruption of the liquid body followed by the ejection of matter. The complete vaporization or even explosion of micrometer-sized drops can result from the linear absorption of laser energy [1-3]. Self-focusing and dielectric breakdown may lead to plasma formation in transparent drops [4-7]. Laser impact has been used to generate liquid motion by vaporization or plasma formation in confined geometries [8-10], sessile drops [11], and biological matter $[12-14]$.

Here, we show how the absorption of laser energy by an unconfined liquid drop induces a rapid phase change (see Fig. 7.1), which in turn controls the propulsion, expansion, and fragmentation of the drop. A key application of the drop shaping by laser impact is found in laser-produced plasma light sources for extreme ultraviolet (EUV) nanolithography $[15,16]$. In these sources the shape, position, and stability of a liquid tin body directly affect the conversion efficiency of liquid tin to a plasma that emits EUV light.

The detailed understanding of the hydrodynamic response of an opaque liquid drop to laser impact poses two fundamental challenges. First, one needs to resolve how momentum is transferred from the laser to the drop. Second, the subsequent deformation dynamics and fragmentation of the drop after impact
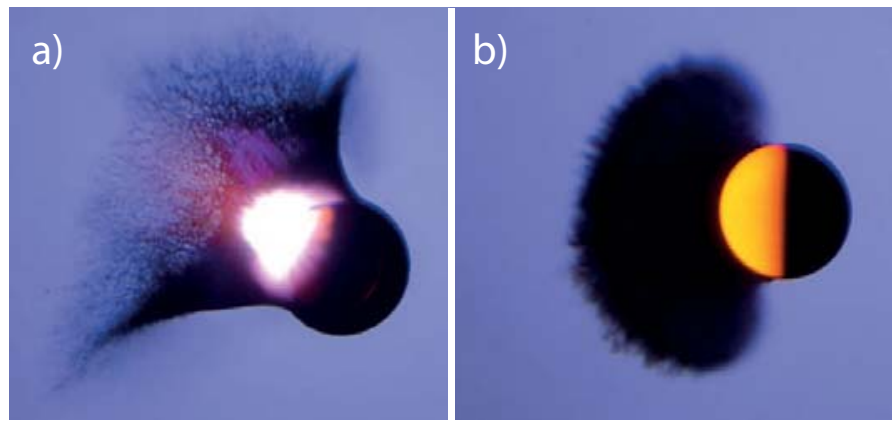

Figure 7.1: Laser-pulses $(\lambda=532 \mathrm{~nm})$ impacting from the left on magentadyed water drops of radius $R_{0}=0.9 \mathrm{~mm}$. Images are taken $30 \mu$ s after impact with a color camera and diffusive backlight illumination. (a) White plasma glow [17] and violent ablation from the drop induced by a focused laser beam. (b) Fluorescence of the dye and ablation at the drop surface due to local boiling induced by a uniform laser irradiation. 
have to be quantified. Although drop impact onto a solid substrate has been studied thoroughly (for a selection, see e.g. Refs. [18-24]), no consensus on the deformation dynamics has yet been reached and only few studies [19, 20, 2527 focused on the fragmentation.

\subsection{Experimental methods}

Our model system consists of a highly-absorbing drop that is hit by a pulsed laser beam. In Fig. 7.2 an overview of the experimental setup is shown. The drop detaches from a capillary, falls, and relaxes to a spherical shape with radius $R_{0}=0.9 \mathrm{~mm}$. While it falls down, the drop masks a photodiode that then generates a reference trigger for the pulsed laser, cameras, and light source. The $\vec{e}_{z}$-axis of the laser beam is aligned orthogonally to the $\vec{e}_{y}$-axis defined by the falling drop and the $\vec{e}_{x}$-axis of the imaging optics. The $x z$-plane in which the laser beam propagates is below the plane of the trigger laser and the pinch-off point at the capillary tube.

The drop consists of dyed water with a density $\rho=998 \mathrm{~kg} / \mathrm{m}^{3}$ and surface tension $\gamma=72 \mathrm{mN} / \mathrm{m}$ assumed to be equal to the properties of pure water. The typical penetration depth of the laser light into the dyed drop is $\delta \sim$ $10 \mu \mathrm{m} \ll R_{0}$ [10], which ensures that the laser energy is absorbed in a thin layer close to the drop surface. The laser-pulse energy is varied between 0 and $120 \mathrm{~mJ}$ by an optical attenuator based on a half-wave plate and a polarizing beam-splitter. The relation between the laser-pulse energy at the drop location and the settings of the attenuator is determined in separate measurements, for which the top beam dump shown in Fig. 7.2 is replaced by an energy meter. A focusing lens decreases the beam diameter to twice the drop size in order to achieve a uniform but high-intensity illumination of the drop. To ensure the drop is placed at the center of the laser beam, the drop position is optimized such that the drop-shape evolution is axisymmetric with respect to $\vec{e}_{z}$ and the propulsion speed is maximum.

The energy $E$ that is actually absorbed by the drop is computed from a beam-profile measurement and ray-tracing. The typical beam fluence $1 \mathrm{~J} / \mathrm{cm}^{2}$ is well below the dielectric breakdown and self-focusing thresholds reported for water with focussed nanosecond laser-pulses $[8,17]$. Consistently, we observe a plasma only when the laser beam is tightly focused inside the drop (Fig. 7.1a, see also $[12,13]$ ), but not for a uniform irradiation (Fig. 7.1b). To visualize the wavelengths in the visible spectrum that are emitted by the drop shown in Fig. 7.1 we use a magenta-colored ink as a dye and a color camera. For 


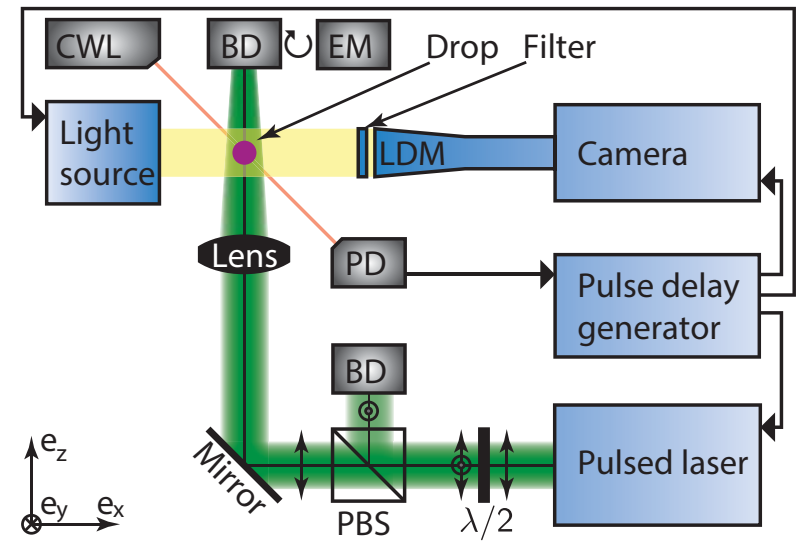

Figure 7.2: Sketch of the experimental setup in top view. The drop (black/magenta ink, IJC-5900/5920 by Sensient) is generated with a capillary tube connected to a syringe pump (PHD2000 by Harvard Apparatus, not shown). A continuous-wave laser (CWL, CPS196 by Thorlabs) and a photo diode (PD, PDA36A by Thorlabs) serve as light barrier to trigger on the falling drop. The lab equipment is synchronized by a high-precision pulse delay generator (BNC575 by Berkeley Nucleonics) according to the indicated signal path. The pulsed laser is a frequency-doubled Nd:YAG laser (Evergreen 140 by Quantel) with a pulse duration $\tau_{\mathrm{p}}=10 \mathrm{~ns}$ emitting at a wavelength $\lambda=532 \mathrm{~nm}$. Attenuation of the laser energy is accomplished by a zero-order half-wave plate $(\boldsymbol{\lambda} / \mathbf{2})$, a polarizing beam splitter (PBS), and a beam dump (BD). The laser-pulse energy is measured by an energy meter (EM, QE12 by gentec-eo). The circle and arrow symbols along the optical axis respectively indicate the S- and $\mathrm{P}$-component of the linearly-polarized laser beam that is focused by a plano-convex lens with a focal length of $f=125 \mathrm{~mm}$. Sideview images (yz-plane) are taken with a long-distance microscope (LDM, 12x Zoom by Navitar), a CMOS camera (FASTCAM SA-X2 by Photron), and a continuous light source (LS-M352A by Sumita). Stroboscopic images are acquired by a CCD camera (PCO1300 by PCO AG) combined with a light source (NANOLITE KL-K by HSPS) that delivers a high-intensity light pulse of $8 \mathrm{~ns}$. A notch filter protects the imaging equipment from scattered laser light. 
all experiments leading to quantitative results we use a black-colored ink to suppress fluorescence.

The post-impact dynamics of the drop (Fig. 7.3a) is observed from a side view $\left(\vec{e}_{z}\right.$-axis in Fig. 7.2) with a long-distance microscope, a high-speed camera operated at a frame rate of 20000 frames per second and a continuous light source. Detailed information in the first microseconds after impact is obtained by operating a camera in stroboscopic mode with a flash lamp that delivers a high-intensity light pulse of $8 \mathrm{~ns}$ (Fig. 7.3b). We record stroboscopic videos by performing a single impact experiment per video frame while changing the time delay between the laser impact and the pulsed light source. For both cameras used the size of the field of view is $16 \times 10 \mathrm{~mm}^{2}$, which yields a pixel resolution of $16 \mu \mathrm{m}$ per pixel.

\subsection{Results \& interpretation}

The drop dynamics for different pulse energies is shown in Fig. 7.3. On impact, the surface of the drop hit by the laser emits a shock wave into the air (Fig. 7.3b). The shock wave is followed by the ejection of a mist cloud of small drops that is visible as a gray-to-black haze in the images and persists for several microseconds. Subsequently, the mist is expelled while the drop propels in the opposite direction (Fig. 7.3a). At the same time the drop flattens and expands in the radial direction before it either retracts, for low pulse energy, or fragments, for large energy.

We quantify the drop motion by measuring the displacement $Z(t)$ of the drop center-of-mass and the drop radius $R(t)$ (defined in Fig. 7.3a) for the first milliseconds after impact. As Fig. 7.4a shows, the drop is propelled at a constant speed $U$ that increases with increasing pulse energy up to $2.0 \mathrm{~m} / \mathrm{s}$. The accompanying deformation of the drop occurs on the inertial time-scale $\tau_{\mathrm{i}}=R_{0} / U \sim 10^{-4}$ to $10^{-3} \mathrm{~s}$ (Fig. $7.4 \mathrm{~b}$ ) and is eventually slowed down by surface tension on the capillary time-scale $\tau_{\mathrm{c}}=\sqrt{\rho R_{0}^{3} / \gamma}=3.5 \mathrm{~ms}$. Both the initial deformation rate $\tau_{\mathrm{i}}^{-1}$ and the maximal extension $R_{\max }$ increase with increasing pulse energy. We emphasize the clear separation of time-scales

$$
\tau_{\mathrm{p}} \ll \tau_{\mathrm{e}} \ll \tau_{\mathrm{i}}<\tau_{\mathrm{c}}
$$

between the successive steps, namely, the laser-pulse, the ejection of matter (on time scale $\tau_{\mathrm{e}} \sim 10^{-5} \mathrm{~s}$ ), the initial deformation of the drop, and its capillary retraction. 


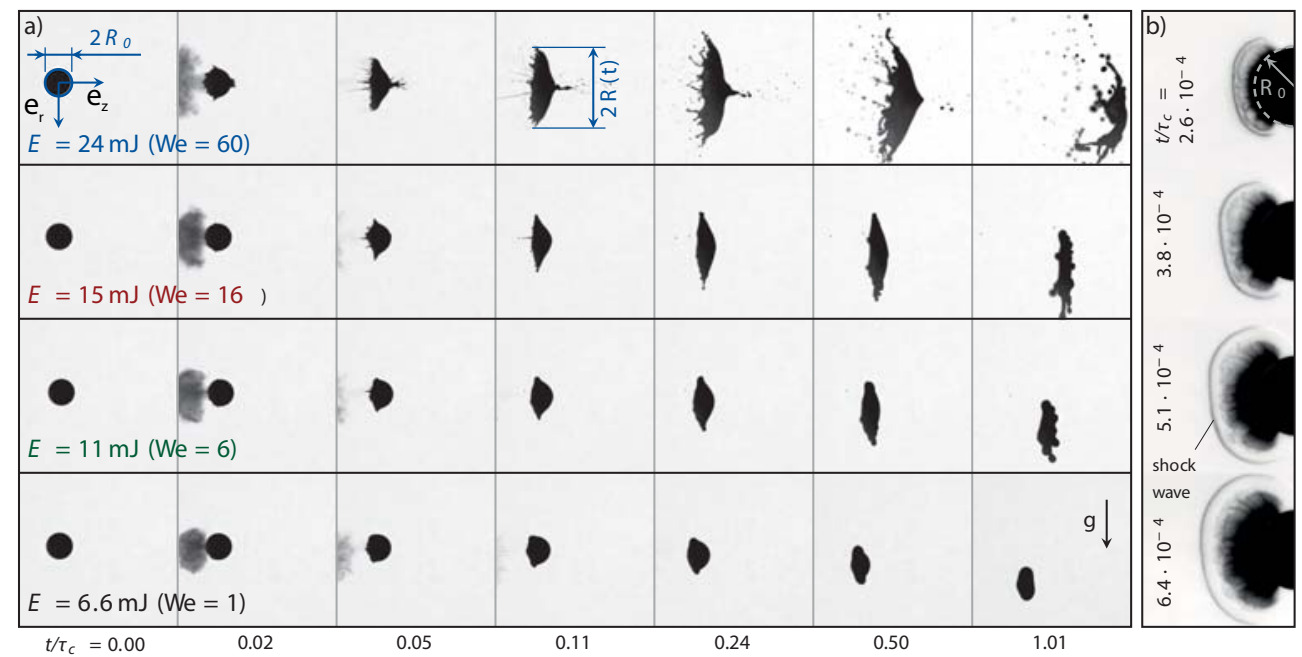

Figure 7.3: Side-view of a dyed water drop with initial radius $R_{0}=0.9 \mathrm{~mm}$ hit at $t=0$ by a laser-pulse propagating from left to right $\left(\vec{e}_{z}\right)$. (a) Drop shape dynamics for pulse energies increasing from bottom to top. $E$ is the energy that is actually absorbed by the drop, We is the Weber number of the propelled drop (see text). The images are taken at a frame rate of 20.000 frames per second $\left(\tau_{\mathrm{c}}=3.5 \mathrm{~ms}\right)$. As the laser ablates the front of the drop a mist cloud is ejected backward $\left(-\vec{e}_{z}\right)$ while the remainder of the drop is propelled forward $\left(\vec{e}_{z}\right)$ and expands radially $\left(\vec{e}_{r}\right)$. For small $E$ the drop retracts after the initial expansion and no break-up occurs. For $E=24 \mathrm{~mJ}$ the edge destabilizes before it retracts and the drop fragments. (b) Close-up view of the drop surface for $E=24 \mathrm{~mJ}$ revealing the shock wave in the air and the mist cloud development at early times (increasing from top to bottom). 

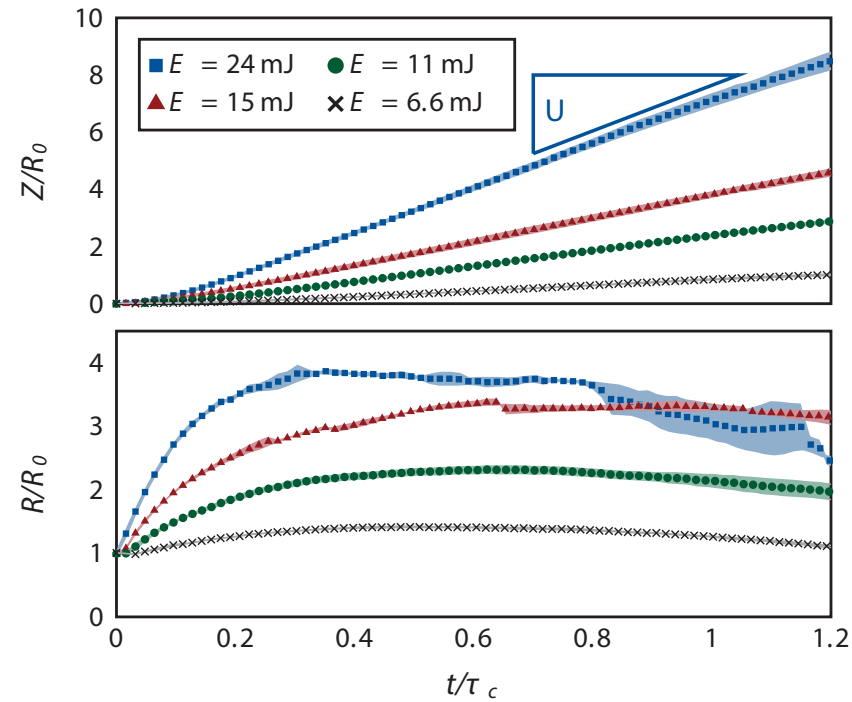

Figure 7.4: Center-of-mass displacement $Z$ (along $\vec{e}_{z}$, see Fig. 7.3) (a) and radial expansion $R$ (b) as a function of time for different absorbed energies. The corresponding image sequences are shown in Fig. 7.3. Each point is averaged over two experiments and the shaded area indicates the difference between the two. The apparent acceleration in $Z$ for $t / \tau_{\mathrm{c}}<0.2$ is an artifact of the method used to determine the center-of-mass position. The large deviation in $R$ for $E=24 \mathrm{~mJ}$ illustrates the statistical nature of the fragmentation. For $E=15 \mathrm{~mJ}$ events of drop ejection from the edge are visible at $t / \tau_{\mathrm{c}}=0.24$, 0.36 and 0.63 .

To explain the relation between the drop propulsion speed, the radial expansion, and the laser energy one needs to understand the mechanism that propels the drop. Surely, both the optical radiation pressure from the laser and the thermal radiation pressure caused by the heating of the drop surface are insignificant $[28,29]$. The motion actually results from the recoil due to the partial vaporization of the drop: since the highly-absorbent dye ensures that the laser energy is absorbed in a superficial layer on one side of the drop, the vapor expulsion is mainly unidirectional and consequently transfers momentum to the remainder of the drop.

The light energy is absorbed by a liquid mass $\sim \rho R_{0}^{2} \delta$ set by the penetration depth of the laser. On the time scale $\tau_{\mathrm{e}}$, both diffusive and radiative heat transfers are negligible (the thermal diffusion length is much smaller than $\delta[30])$. Since the beam profile is flat, and neither the focusing due to the 
drop interface curvature nor nonlinear optical effects (self-focusing or electric breakdown) are significant, we consider the energy deposition in the superficial layer to be close to uniform. This energy is sufficient to heat the liquid from the ambient temperature $T_{0}=293 \mathrm{~K}$ to the boiling temperature $T_{\mathrm{b}} \simeq 393 \mathrm{~K}$, but not to vaporize all of it: only a certain fraction $\beta$ actually vaporizes. The energy balance therefore reads $E \sim \rho R_{0}^{2} \delta\left[c_{\mathrm{v}}\left(T_{\mathrm{b}}-T_{0}\right)+\beta \Delta H\right]$, where $c_{\mathrm{v}}=4.0 \mathrm{~kJ} /(\mathrm{kg} \mathrm{K})$ and $\Delta H=2.25 \mathrm{MJ} / \mathrm{kg}$ are, respectively, the specific heat capacity and latent heat of vaporization of the liquid.

In all our experiments a mist cloud is observed, which is a clear signal of a local boiling of the drop. We therefore assume that to get propulsion, a threshold energy $E_{\text {th }} \sim \rho R_{0}^{2} \delta c_{\mathrm{v}}\left(T_{\mathrm{b}}-T_{0}\right) \approx 3 \mathrm{~mJ}$ has to be absorbed by the superficial layer to heat the liquid to the boiling point, which is in good agreement with the threshold for propulsion observed in our experiments (Fig. 7.5a). Any additional energy deposited in the superficial layer is used to vaporize a mass of liquid $m \sim \beta \rho R_{0}^{2} \delta \sim\left(E-E_{\mathrm{th}}\right) / \Delta H$. An upper limit for the proposed scaling is given by $E / E_{\mathrm{th}} \sim 1+\Delta H /\left[c_{\mathrm{v}}\left(T_{\mathrm{b}}-T_{0}\right)\right] \approx 8$, in which case the absorbed energy is sufficient to evaporate the entire heated liquid layer (i.e. $\beta=1$ ). Any increase in $E$ beyond this point would lead to a superheated or even a critical phase, in which case the opaque mist cloud would not be observed [13].

For $0<\beta<1$, which is the case of our experiments, the remaining part of the heated layer that is not vaporized is expelled as a mist of small drops. We assume that the liquid vaporizes at $T_{\mathrm{b}}$ and that the vapor is expelled at the thermal speed $u=\sqrt{k_{b} T_{\mathrm{b}} / \mu} \approx 400 \mathrm{~m} / \mathrm{s}$, where $k_{b} \simeq 1.38 \times 10^{-23} \mathrm{~J} / \mathrm{K}$ is the Boltzmann constant and $\mu=2.99 \times 10^{-26} \mathrm{~kg}$ is the molecular mass of water. This expelled vapor propels the remainder of the drop. Momentum conservation $m u=\rho R_{0}^{3} U$ yields

$$
U \sim \frac{E-E_{\mathrm{th}}}{\rho R_{0}^{3} \Delta H} u,
$$

that is, an increase in $U$ proportional to that in $E$. Figure 7.5a shows that this scaling argument, with a prefactor of 0.4 , is in good agreement with our experimental data.

With a description of the propulsion at hand, we now turn to the drop deformation. The expansion dynamics is directly affected by surface tension, which promotes the retraction and possibly the fragmentation of the drop. The key parameter describing the expansion is therefore the Weber number of the motion induced by the laser We $=\rho R_{0} U^{2} / \gamma$, which compares the drop displacement kinetic energy to its surface energy. In our experiments $1 \leq$ We $\leq 60$. The impulsive acceleration of our drop from 0 to $U$ is similar to the 
impulsive stop of a drop impacting a solid with velocity $U$. We therefore use the momentum-based scaling derived by $[18,20]$ for drop impact on solids to express the maximal radial expansion

$$
\frac{R_{\max }-R_{0}}{R_{0}} \sim \mathrm{We}^{1 / 2} \sim \sqrt{\frac{\rho R_{0} u^{2}}{\gamma}} \frac{E-E_{\mathrm{th}}}{\rho R_{0}^{3} \Delta H},
$$

in which the expression in terms of $E$ directly comes from (2). Expression (3), with a prefactor of 0.6 , is in good agreement with our experimental data up to We $\sim 40$, when the drop starts fragmenting and the maximum expansion saturates (see Fig. 7.5b). The scaling (3) has already been observed for drop impact onto solid substrates with negligible friction [20]. The present setup is however fundamentally different since, as mentioned above, the typical impact timescale, during which the drop accelerates, is decoupled from the inertial timescale: $\tau_{\mathrm{e}} \ll \tau_{\mathrm{i}}$.

\subsection{Numerical results}

To confirm that the interaction of the laser-pulse with the drop can be modeled as a short recoil-pressure pulse exerted on the drop surface, we perform Boundary Integral (BI) simulations [31-33]. We assume that the flow inside the drop is inviscid, irrotational, and incompressible, and solve the resulting Laplace equation for the flow potential. The method assumes axisymmetry and therefore cannot be used to study the eventual fragmentation of the drop, but it does capture the initial phase of the drop deformation.

The laser-pulse is modeled by applying a pressure boundary condition at the drop surface for a time duration $\tau_{\mathrm{e}} \ll \tau_{\mathrm{i}}$. We use a Gaussian pressure profile with a length-scale based on the measured laser-beam profile and a pressure scale set to match the propulsion velocity observed in the experiment. This pressure scale is prescribed by the momentum conservation $p R_{0}^{2} \tau_{\mathrm{e}} \sim \rho R_{0}^{3} U$ (the prefactor can be obtained analytically [34]). From (7.2) this recoil pressure can readily be expressed in terms of the absorbed energy.

The numerical drop shape evolution is shown in Fig. 7.6. It illustrates the added value of the simulations: not only the two-dimensional projection of the drop shape, but also the spatial and temporal evolution of the sheet thickness can be extracted, which is crucial when it comes to study fragmentation [20]. Moreover, Fig. 7.6 shows that the BI model quantitatively predicts the radial drop expansion observed for different Weber numbers. This confirms that a pressure pulse applied at the drop surface for a time much shorter than the 

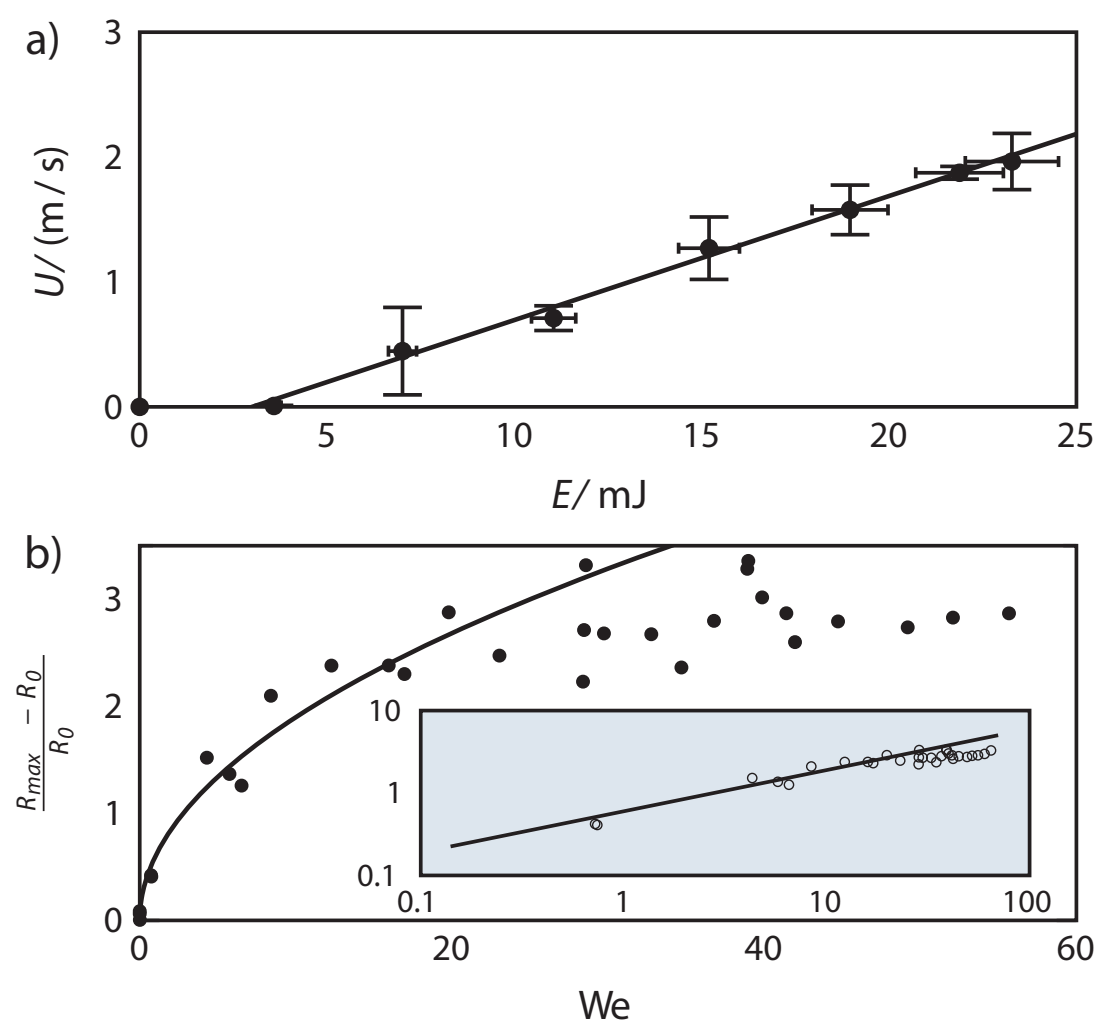

Figure 7.5: (a) Propulsion speed of the drop as a function of the absorbed laser energy. Each point represents at least four experiments, the error bars indicate the standard deviation. The solid line is equation (7.2) with a prefactor of 0.4. (b) Maximal relative expansion $R_{\max } / R_{0}-1$ for individual experiments as a function of the Weber number in linear and logarithmic (inset) scales. The solid line is equation (7.3) with a prefactor of 0.6. For large Weber numbers a saturation is observed due to the fragmentation of the sheet. 


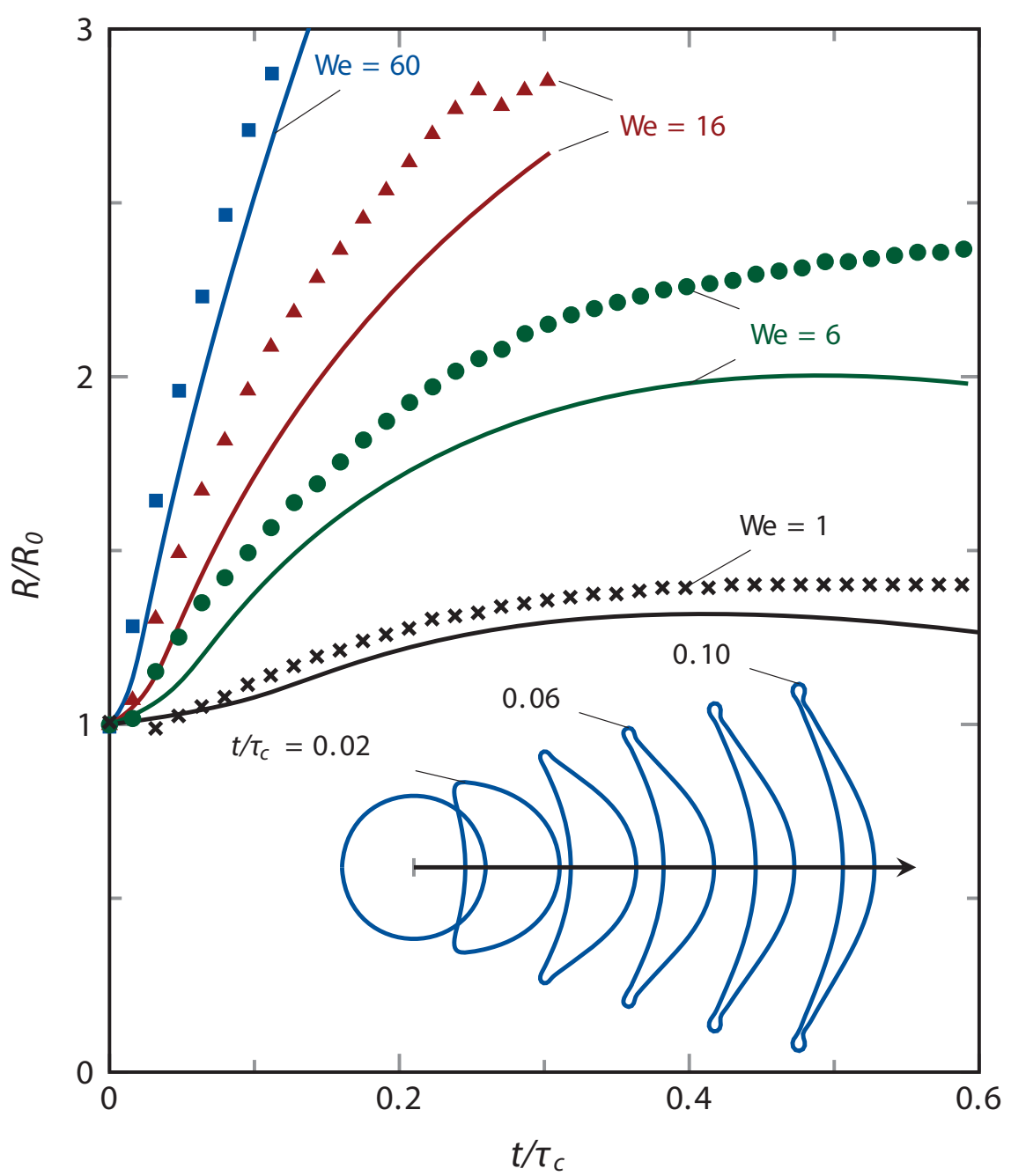

Figure 7.6: Radial expansion of the drop: experiments (markers) and BI simulations (solid lines). The corresponding image sequences are shown in Fig. 7.3. The inset shows the drop shape evolution from $\mathrm{BI}$ for $\mathrm{We}=60$ with an exaggerated center-of-mass displacement to separate the successive shapes. The simulations are stopped when the local sheet thickness becomes too thin to resolve the dynamics accurately. 

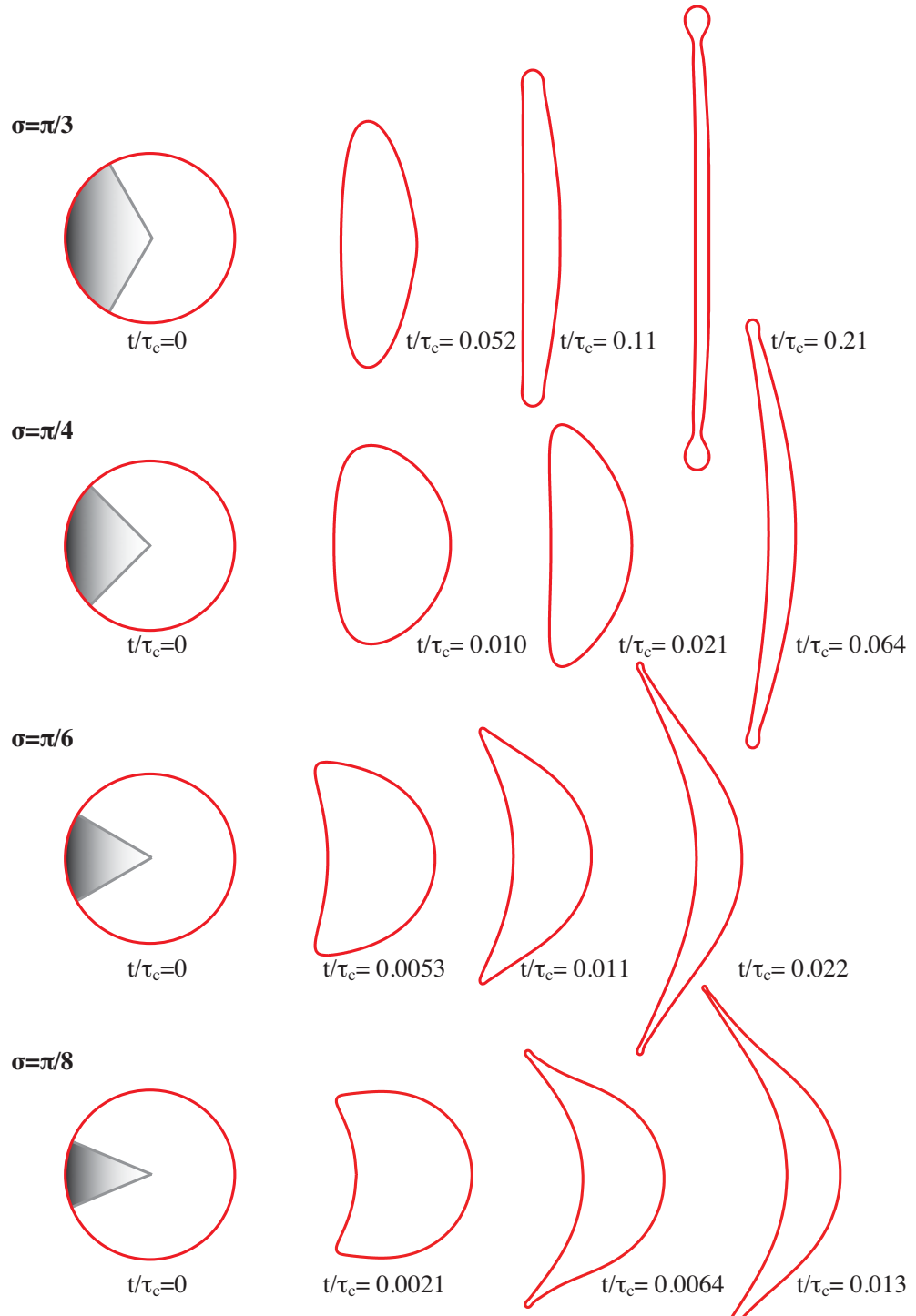

$\mathrm{t} / \tau_{\mathrm{c}}=0.022$

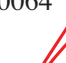

Figure 7.7: Drop contours obtained from the BI simulations at different points in time showing the drop shape evolution for four different pressure-pulse widths (top to bottom): $\sigma=\pi / 3, \pi / 4, \pi / 6$, and $\pi / 8$, for We $=790$. To clearly illustrate the drop shape evolution for each pulse width each sequence of drop contours is plotted at different times, owing to the differences in expansion speeds. 
hydrodynamic time scales $\left(\tau_{\mathrm{i}}\right.$ and $\left.\tau_{\mathrm{c}}\right)$ is indeed sufficient to describe the hydrodynamic response of a drop to the impact of a laser-pulse. For completeness we also show the results for different widths $\sigma$ of the Gaussian pressure pulse. Fig. 7.7 shows the drop contours for $\sigma=\pi / 3, \pi / 4, \pi / 6$ and $\pi / 8$ for $\mathrm{We}=790$. Clearly, a smaller $\sigma$ (focussed laser beam) leads to a larger expansion speed, thinner sheet with non-uniform thickness and a more curved drop shape.

\subsection{Conclusions}

We have shown that an opaque free-falling drop hit by a laser-pulse propels and expands until fragmentation occurs. In the present case the laser energy is absorbed in a superficial layer of the drop such that the deposited energy per unit mass $E / \rho R_{0}^{2} \delta \sim 0.1$ to $1 \mathrm{MJ} / \mathrm{kg}$ is comparable to the specific latent heat of vaporization. As a consequence, drop motion is induced by the recoil due to vaporization on the face of the drop that is hit by the laser. This results in a propulsion speed and a maximal radius of expansion that are both proportional to the pulse energy. The expansion dynamics is limited by surface tension and is similar to that of a drop impacting a solid, although with a laserpulse momentum transfer takes place on a much shorter time scale. Laserinduced drop fragmentation and the influence of the beam focusing require detailed studies and are left for future work [34]. All results reported here should transpose directly to the shaping of liquid tin drops in EUV light sources. In a regime where a plasma is generated the propulsion mechanism may change, however, the Weber number remains the key parameter governing the hydrodynamic response.

\section{A Measurement of energy absorption}

We first measure the laser beam profile at the drop location (Fig. 7.8). To avoid saturation of the beam profiler we attenuate the laser energy by a factor of $10^{6}$ with two wedged windows and a reflective neutral-density filter. To determine the fraction of the light that is refracted into the drop at the airliquid interface we use ray tracing (valid for $R_{0} \gg \lambda=532 \mathrm{~nm}$ ). Since the dye is highly absorbent $\left(\delta \ll R_{0}\right)$, we neglect any light transmission through the drop. The initial condition of the ray tracing is set by the focal length of the focusing lens and the position of the drop in the laser beam (see Fig. 7.2 of this chapter). The distribution of rays in space and the energy attributed to each ray is chosen such that the set of rays resembles the measured beam profile. 


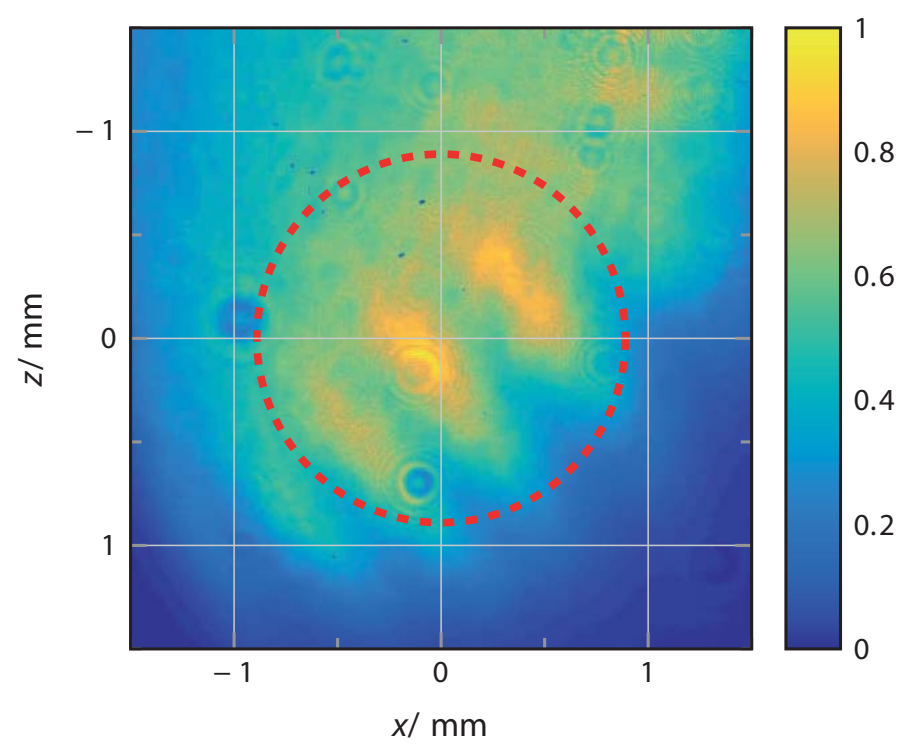

Figure 7.8: Normalized fluence of the laser beam measured at the drop location by a CCD beam profiler (BC106-VIS by Thorlabs). The profile depicted is the mean result of 10 single measurements. The dotted line indicates the position of the drop during the laser-impact experiments.

As a result, we estimate that in our experiments $20 \%$ of the laser-pulse energy is absorbed by the drop.

\section{B Measurement of drop displacement}

For each recorded image we determine the center-of-mass position assuming rotational symmetry of the two-dimensional drop shape. Thereby, we ignore the concavity of the drop shape, which introduces an error in the determination of the centre-of-mass position. This causes an apparent acceleration on the time-scale $\tau_{\mathrm{i}}$ in $Z$ (visible in Fig. 7.4).

To confirm that this acceleration is an artificial result of our algorithm, we use the results from the BI simulations. In BI the full three-dimensional axisymmetric shape of the drop is known, and hence the exact centre-of-mass displacement can be determined (dashed, blue line in Fig. 7.9). In addition, we compute the centre-of-mass displacement based on the two-dimensional projection of the BI data using the same procedure as we apply to the ex- 


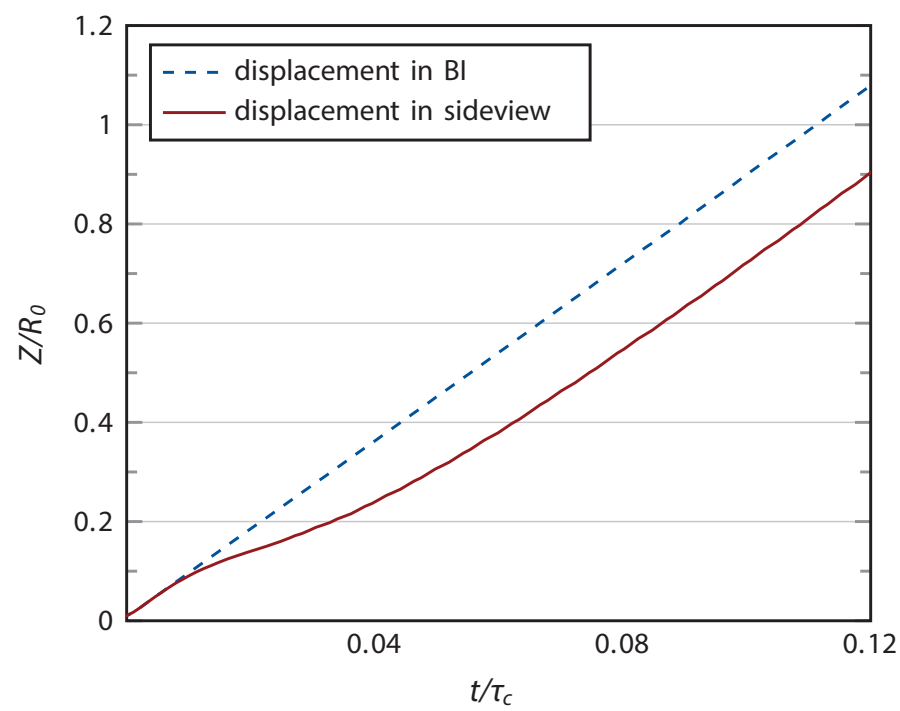

Figure 7.9: Center-of-mass displacement $Z$ of the drop (along $\vec{e}_{z}$, see Fig. 7.2) as a function of time in the BI simulation for $E=24 \mathrm{~mJ}$. The blue dashed line represents the actual centre-of-mass displacement, whereas the red solid line is the centre-of-mass displacement based on the two-dimensional projection of the simulated drop shape, which shows an artificial deceleration.

perimental side-view images (solid, red line in Fig. 7.9). The centre-of-mass determined from the two-dimensional projection shows an artificial deceleration as soon as the drop shape becomes concave. However, Fig. 7.9 also shows that the final speed of the deforming drop is not affected by the apparent initial deceleration. Therefore, the error introduced by considering the projected drop shape only affects the initial deceleration of the centre-of-mass, not its final speed.

\section{References}

[1] R. G. Pinnick, A. Biswas, R. L. Armstrong, S. G. Jennings, J. D. Pendleton, and G. Fernández, "Micron-sized droplets irradiated with a pulsed CO2 laser: measurement of explosion and breakdown thresholds", Appl. Opt. 29, 918-925 (1990).

[2] P. Kafalas and A. P. Ferdinand, "Fog droplet vaporization and fragmentation by a 10.6- $\mu$ m laser pulse", Appl. Opt. 12, 29-33 (1973). 
[3] P. Kafalas and J. Herrmann, "Dynamics and energetics of the explosive vaporization of fog droplets by a 10.6- $\mu$ m laser pulse", Appl. Opt. 12, $772-775$ (1973).

[4] J.-Z. Zhang, J. K. Lam, C. F. Wood, B.-T. Chu, and R. K. Chang, "Explosive vaporization of a large transparent droplet irradiated by a high intensity laser", Appl. Opt. 26, 4731-4737 (1987).

[5] C. Favre, V. Boutou, S. C. Hill, W. Zimmer, M. Krenz, H. Lambrecht, J. Yu, R. K. Chang, L. Woeste, and J.-P. Wolf, "White-light nanosource with directional emission", Phys. Rev. Lett. 89, 030401 (2002).

[6] Y. Geints, A. Kabanov, G. Matvienko, V. K. Oshlakov, A. A. Zemlyanov, S. S. Golik, and O. A. Bukin, "Broadband emission spectrum dynamics of large water droplets exposed to intense ultrashort laser radiation", Opt. Lett. 35, 2717-2719 (2010).

[7] A. Lindinger, J. Hagen, L. D. Socaciu, T. M. Bernhardt, L. Wóste, D. Duft, and T. Leisner, "Time-resolved explosion dynamics of h2o droplets induced by femtosecond laser pulses", Appl. Opt. 43, 5263 (2004).

[8] A. Vogel, S. Busch, and U. Parlitz, "Shock wave emission and cavitation bubble generation by picosecond and nanosecond optical breakdown in water", J. Acoust. Soc. Am. 100, 148:165 (1996).

[9] C. Sun, E. Can, R. Dijkink, D. Lohse, and A. Prosperetti, "Growth and collapse of a vapour bubble in a microtube: the role of thermal effects", J. Fluid Mech. 632, 5-16 (2009).

[10] Y. Tagawa, N. Oudalov, C. W. Visser, I. R. Peters, D. van der Meer, C. Sun, A. Prosperetti, and D. Lohse, "Highly focused supersonic microjets", Phys. Rev. X 2, 031002 (2012).

[11] S. T. Thoroddsen, K. Takehara, T. G. Etoh, and C. D. Ohl, "Spray and microjets produced by focusing a laser pulse into a hemispherical drop", Phys. Fluids 21, 112101 (2009).

[12] A. Vogel and V. Venugopalan, "Mechanisms of pulsed laser ablation of biological tissues", Chem. Rev. 103, 577-644 (2003).

[13] I. Apitz and A. Vogel, "Material ejection in nanosecond Er:YAG laser ablation of water, liver, and skin", Appl. Phys. A 81, 329-338 (2005). 
[14] V. Horneffer, N. Linz, and A. Vogel, "Principles of laser-induced separation and transport of living cells", J. Biomed. Opt 12, 054016-054016-13 (2007).

[15] H. Mizoguchi, T. Abe, Y. Watanabe, T. Ishihara, T. Ohta, T. Hori, T. Yanagida, H. Nagano, T. Yabu, S. Nagai, G. Soumagne, A. Kurosu, K. M. Nowak, T. Suganuma, M. Moriya, K. Kakizaki, A. Sumitani, H. Kameda, H. Nakarai, and J. Fujimoto, "1st generation laser-produced plasma source system for hvm euv lithography", Proc. SPIE 7636, 76308 (2010).

[16] V. Banine, K. Koshelev, and G. Swinkels, "Physical processes in euv sources for microlithography", J. Phys. D: Appl. Phys. 44, 253001 (2011).

[17] P. Kennedy, D. Hammer, and B. Rockwell, "Laser-induced breakdown in aqueous media", Prog. Quant. Electron. 21, 155-248 (1997).

[18] C. Clanet, C. Béguin, D. Richard, and D. Quéré, "Maximal deformation of an impacting drop", J. Fluid Mech. 517, 199-208 (2004).

[19] A. Yarin, "Drop impact dynamics: splashing, spreading, receding, bouncing...", Annu. Rev. Fluid Mech. 38, 519-592 (2006).

[20] E. Villermaux and B. Bossa, "Drop fragmentation on impact", J. Fluid Mech. 668, 412-435 (2011).

[21] P. A. Tsai, M. Hendrix, R. Dijkstra, L. Shui, and D. Lohse, "Microscopic structure influencing macroscopic splash at high weber number", Soft Matter 7, 11325-11333 (2011).

[22] C. W. Visser, P. E. Frommhold, S. Wildeman, R. Mettin, D. Lohse, and S. Chao, "Dynamics of high-speed micro-drop impact: numerical simulations and experiments at frame-to-frame times below 100ns", Soft Matter 11(9), 1708-1722 (2014).

[23] T. Tran, H. J. J. Staat, A. Susarrey-Arce, T. C. Foertsch, A. V. Houselt, H. J. G. E. Gardeniers, A. Prosperetti, D. Lohse, and C. Sun, "Droplet impact on superheated micro-structured surfaces", Soft Matter 1-11 (2013).

[24] G. Riboux and J. M. Gordillo, "Experiments of drops impacting a smooth solid surface: a model of the critical impact speed for drop splashing", Phys. Rev. Lett. 113, 024507 (2014). 
[25] L. Xu, L. Barcos, and S. R. Nagel, "Splashing of liquids: Interplay of surface roughness with surrounding gas", Phys. Rev. E 76, 066311 (2007).

[26] E. Villermaux, "Fragmentation", Annu. Rev. Fluid Mech. 39, 419-446 (2007).

[27] E. Villermaux and B. Bossa, "Single-drop fragmentation determines size distribution of raindrops", Nat. Phys. 5, 697-702 (2009).

[28] The typical impulse $I$ exerted on the drop by the $3 \%$ reflected light from the surface scales as $I \sim E_{\mathrm{r}} / c$, with $E_{\mathrm{r}}$ the energy of the reflected light and $c$ the speed of light. This impulse would yield a typical drop speed $U \sim 10^{-7} \mathrm{~m} / \mathrm{s}$. The impulse due to thermal radiation from the hot drop surface scales as $I \sim \epsilon \sigma T^{4} R_{0}^{2} \tau_{p} / c$ with $\epsilon$ the emissivity and $\sigma$ the StefanBoltzmann constant. This impulse would yield $U \sim 10^{-14} \mathrm{~m} / \mathrm{s}$.

[29] J.-P. Delville, M. de Saint Vincent, R. Schroll, H. Chraïbi, B. Issenmann, R. Wunenburger, D. Lasseux, W. Zhang, and E. Brasselet, "Laser microfluidics: fluid actuation by light", J. Opt. A-Pure Appl. Op. 11, 034015 (2009).

[30] On the time scale of vapor ejection $\tau_{\mathrm{e}}$ heat typically diffuses over a length $\sqrt{\kappa \tau_{\mathrm{e}}} \sim 10^{-6} \mathrm{~m} \ll \delta$, where $\kappa$ is the thermal diffusivity of water .

[31] R. Bergmann, D. van der Meer, S. Gekle, A. van der Bos, and D. Lohse, "Controlled impact of a disk on a water surface: cavity dynamics", J. Fluid Mech. 633, 381-409 (2009).

[32] S. Gekle, I. R. Peters, J. M. Gordillo, D. van der Meer, and D. Lohse, "Supersonic air flow due to solid-liquid impact", Phys. Rev. Lett. 104, 024501 (2010).

[33] W. Bouwhuis, R. C. A. van der Veen, T. Tran, D. L. Keij, K. G. Winkels, I. R. Peters, D. van der Meer, C. Sun, J. H. Snoeijer, and D. Lohse, "Maximal air bubble entrainment at liquid-drop impact", Phys. Rev. Lett. 109, 264501 (2012), See Chapter 2 of this thesis.

[34] H. Gelderblom, H. Lhuissier, A. L. Klein, W. Bouwhuis, D. Lohse, E. Villermaux, and J. H. Snoeijer, "Drop deformation by laser-pulse impact", in preparation . 


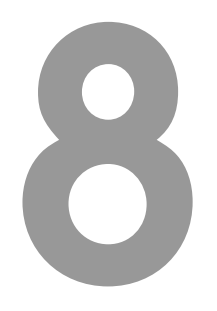

\title{
Effect of surface wettability on inertial pouring flows
}

\begin{abstract}
A liquid poured from a curved solid surface can separate as a steady jet or sheet, or trickle down along the solid surface. It was shown by Duez et al. [Phys. Rev. Lett. 104, 084503 (2010)] that surface wettability controls the separation of an inertial flow from a solid surface to an unexpected degree, which was further motivated by an inertial-capillary adhesion model. In this chapter we extend the analysis by a control volume calculation that takes into account the velocity profile within the flowing layer, supported by Boundary Integral potential flow simulations, and the detailed capillary forces induced by the local curvatures of the sheet. Our analysis captures the appearance of a critical Weber number below which no steady separated solutions can be sustained. We investigate the dependence of the critical Weber number on the wettability and sharpness of the edge of the curved solid, and recover the key experimental trends.
\end{abstract}

${ }^{*}$ Submitted as: W. Bouwhuis, J.H. Snoeijer, "Effect of surface wettability on inertial pouring flows". 


\subsection{Introduction}

The so-called 'teapot effect' is a daily life phenomenon that will be recognized by everyone. When a liquid is poured too slowly from the (hydrophilic) nose of a teapot, a bottle or a beaker, the liquid has the tendency to run down along the underside of the spout, as depicted in Fig. 8.1a. This remarkable effect already received attention in the 1950s [1, 2], and was explained by Keller and Vanden-Broeck as a purely hydrodynamic (Bernoulli) principle [2-4]. Since there is a difference in flow velocity between the top and bottom of the liquid film, the pressure is lowest directly above the spout lip; sufficiently low that the flow is pushed further down along the convex solid. In this situation the liquid film completely 'trickles' around the solid, and exact potential flow solutions were found in which the edge of the solid was treated as perfectly sharp [3, 4]. After trickling, a possible separation from the solid could be induced by gravity.
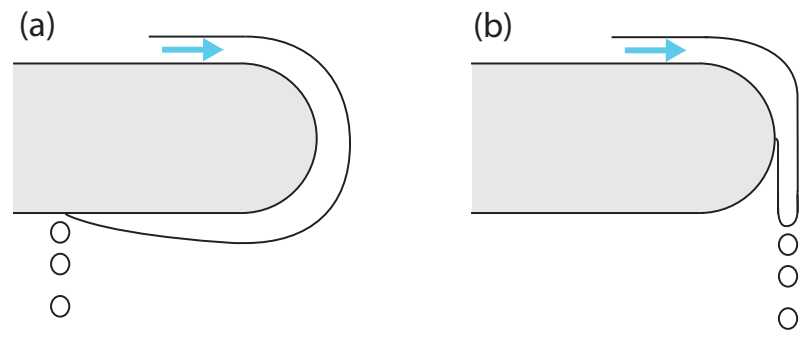

Figure 8.1: Problem sketch. (a) Water flow trickling around the curved (hydrophilic) solid surface, known as the 'teapot effect'. (b) The water repellence of the solid (or the flow speed of the liquid) overcomes the trickling behavior.

However, one can overcome the trickling scenario by increasing the flow speed, such that the liquid jet or sheet separates from the solid with an angle, as is sketched in Fig. 8.1b. Simulations of pouring flows have revealed complicated dynamics, particularly when the Reynolds number of the flow becomes of order unity [5,6]. Moreover, Duez et al. [7] found that surface wettability controls the flow separation to an unexpected degree, also at large Reynolds numbers. This was also confirmed by recent experiments that aim to develop controlled overflow by surface manipulation [8]. Since wettability is determined on a microscopic scale, this highlights the importance of multiple length scales in the problem of pouring flows. In some sense, the experiments and analysis by Duez et al. have revealed a relatively simple framework for 
understanding the various regimes of pouring flow. The experiments were performed using an axisymmetric set-up that is the top part of the 'liquid bell' geometry [9-11], for which the relevant parameters are the flow speed, the wettability of the solid, and the radius of curvature at the edge of the impacter. The transition between inertia-induced sheet separation and trickling appeared at well-defined Weber numbers, whose critical values depend on the contact angle and sharpness of the solid edge. Using a scaling argument for the horizontal momentum balance, Duez et al. predicted a dependency on the flow speed of the liquid and the wettability and sharpness of the solid surface, capturing the main experimental trends - though surprisingly, the experimental trends by Dong et al. [8] suggest a different dependence on the curvature of the edge (linear instead of quadratic). The modeling approach has so far been limited to a force balance in the horizontal (not in the vertical) direction and it has remained a challenge to explicitly capture the origin of the trickling transition. In addition, it is not clear how these results relate to the analytical potential flow solutions $[3,4]$, which excluded the effect of surface tension and finite edge-curvature.

In this chapter, we will perform a control volume analysis for inertial pouring flows over a solid edge of finite curvature, by taking into account the capillary forces induced by the shape of the meniscus and the velocity profile within the liquid. By releasing some geometric constraints imposed in [7], this will allow for a force balance in the horizontal and vertical direction. The model is solved for varying flow parameters and geometric parameters, and we show how this indeed leads to the appearance of a critical Weber number for the trickling transition. In Sec. 8.2, we will introduce the relevant parameters in the problem and the basic assumptions. This results into a set of coupled equations that provide a prediction for the separation angle. Our main findings will be presented in Sec. 8.3, where we give both numerical and asymptotic predictions for the critical Weber number. The results will be summarized and compared to experiments in Sec. 8.4.

\subsection{Model}

\subsubsection{Definitions and assumptions}

A sketch of the problem and the relevant parameters is given in Fig. 8.2a. Like Ref. [7], we treat the flow as two-dimensional and define a horizontal $\mathbf{x}$ direction, and a vertical $\mathbf{y}$ direction. We assume a steady, laminar, irrotational flow with high Reynolds number. In that case the flow can be considered 
uniform both at the inflow above the solid and within the separated sheet, with velocity $U$ and the film thickness is $h$. The edge of the solid has a circular shape, characterized by the radius of curvature $r_{i}$. We assume the sheet separates at an angle $\alpha$ with respect to the horizontal direction, and an important part of the analysis is to determine this angle. The sheet separates from the solid at the position angle $\beta$ defined with respect to the vertical axis. Note that, in general, $\alpha \neq \beta$ and we treat these angles as independent parameters. At the separation point, a small capillary meniscus is formed, which has a radius of curvature $r_{m}$, which is set by the Young-Laplace pressure difference over the free surface $[12,13]$. Locally, the circle formed by the meniscus crosses the $r_{i}$-circle with the contact angle $\theta_{0}$, which is how the surface wettability enters the analysis. Note that $r_{m}$ is typically much smaller than $r_{i}$ (Fig. 8.2 not drawn to scale).

(a)
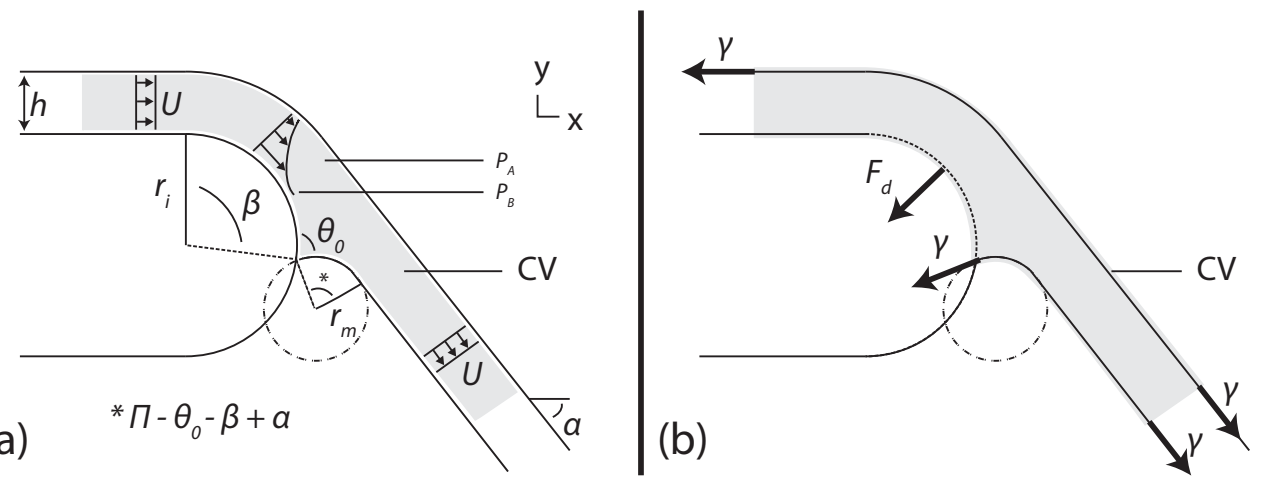

Figure 8.2: Flow around the edge of a solid and two different definitions of the Control Volume (CV), indicated by the gray area. (a) Definition sketch and the Control Surface (CS) located just inside the liquid. A liquid film with thickness $h$, which has a uniform flow velocity $U$, bends around a solid surface with radius of curvature $r_{i}$. $\theta_{0}$ is the solid-liquid contact angle. $\alpha$ is the global deflection angle of the separated liquid sheet with respect to the horizontal $(\mathbf{x})$ direction, and $\beta$ is the angular width of the wetted fraction of the solid. $\alpha$ and $\beta$ are a priori independent. $r_{m}$ is the radius of curvature of the circle formed by the meniscus. The figure is not drawn to scale, typically $r_{m} \ll r_{i}$. The value of the marked angle $*$ is $\pi-\theta_{0}-\beta+\alpha$. (b) The force balance and the CS located just outside the liquid. The forces, indicated by the arrows, respectively are the capillary forces acting in the interface (denoted by $\gamma$ ), and the resultant force $F_{d}$ induced by the pressure difference over the sheet $\left(P_{A}-P_{B}\right)$. 
Assuming inviscid flow, the introduced parameters can be expressed by three dimensionless control parameters, which are the Weber number We $=$ $\rho U^{2} h / \gamma$, where $\rho$ and $\gamma$ are the density and liquid-gas surface tension of the liquid, respectively, the ratio $\tilde{r}_{i}=r_{i} / h$, and the contact angle $\theta_{0}$. For characteristic values around $U \sim 1 \mathrm{~m} / \mathrm{s}$ and $h \sim 0.1-1 \mathrm{~mm}$, the Weber number for water is of order 1 to 10 . The corresponding Reynolds numbers are about $10^{2}-10^{3}$, so we can indeed assume laminar flow and neglect viscosity.

Clearly, the formulation presented here closely follows Duez et al. [7], though some notable differences appear. Importantly, we release the geometric constraint that the tangent of the sheet (with angle $\alpha$ with respect to the vertical direction), is also a tangent line to the edge of the solid (i.e. the circle of radius $r_{i}$ ). In this manner we can treat $\alpha$ and $\beta$ as two independent parameters, and the momentum balance can be maintained in both $x$ and $y$ directions. Other minor differences appear below, when estimating the forces acting on the control volume.

We will solve the deflection angle $\alpha$ of the liquid sheet as the result of the hydrodynamic and capillary forces using linear momentum conservation. The mechanism responsible for the liquid sheet bending is that the velocity profile over the curved solid is not uniform, contrarily to the inflow and the outflow of the control volume indicated in Fig. 8.2. This velocity profile implies a pressure difference across the film, which induces a hydrodynamic force, $F_{d}$, exerted by the solid on the liquid, pulling the liquid along the curved edge. This is sometimes referred to as the Coanda effect [14]. The resultant capillary force in the small meniscus is denoted as $F_{m e n}$, while the effect of surface tension over the top of the curved sheet yields $F_{\text {top }}$. Importantly, we can define the $\mathrm{CV}$ in two ways, indicated by Fig. 8.2a and b, respectively. In Fig. 8.2a, the Control Surface (CS), is located just inside the liquid; in Fig. 8.2b the CS is located just outside the liquid. In the first case, the capillary force can be evaluated by integrating the Young-Laplace pressure over the control surfaces; in the second case, there is no normal force working on these surfaces, but the surface tension acts parallel on the edge of the liquid domain. Both points of view of course result into the same final equations and the resultant forces are indicated by the arrows in Fig. 8.2b. The resulting momentum balance for the $\mathrm{x}$ direction reads

$$
\rho U^{2} h(\cos \alpha-1)=F_{d, x}+F_{m e n, x}+F_{t o p, x},
$$

while for the $\mathbf{y}$ direction we have: 


$$
-\rho U^{2} h \sin \alpha=F_{d, y}+F_{m e n, y}+F_{t o p, y} .
$$

Here we used that the Control Volume (CV) contains the full bending of the stream, such that the left and right boundary of the $\mathrm{CV}$ are located where the flow is uniform.

It is instructive to consider the momentum balance in the context of the exact solutions by Keller \& Vanden-Broeck [2-4]. These are obtained by treating the solid as a perfectly sharp edge in the absence of capillary effects. This effectively corresponds to $\mathrm{We}=\infty, \tilde{r}_{i}=0$, while the angles $\theta_{0}$ and $\beta$ are not defined in this limit. It was found that analytical solutions exist for each value of $\alpha$; one could use the momentum balance $(8.1,8.2)$ without the capillary forces $\left(F_{m e n}=F_{t o p}=0\right)$ to determine the dynamical force $F_{d}$, for each value of $\alpha$. Importantly, however, this analysis does not lead to a selection of $\alpha$. A selection of $\alpha$ does appear at finite values of We and $\tilde{r}_{i}$, one thus needs explicit expressions for all terms in Sys. $(8.1,8.2)$, as will be developed below. In Sec. 8.2.2, we investigate the velocity profile and the pressure distribution in the liquid, from which we compute the various forces in Sec. 8.2.3. The resulting set of equations will be presented in Sec. 8.2.4.

\subsubsection{Flow profiles and pressures}

To compute the pressure distribution in the liquid we require the velocity profile inside the flowing liquid, for a given geometry characterized by $\tilde{r}_{i}$. Here, we focus on the case $\tilde{r}_{i} \gg 1$ for which the flow will evolve towards concentric 'circular' streamlines around the circular edge of the solid [10]. Under the assumption of potential flow, this corresponds to a free vortex with a tangential flow velocity $u \sim 1 / r$. Such a profile is quite different from the corner solutions for $\tilde{r}_{i} \ll 1$, and the resulting pressure distribution is expected to be quite different.

We therefore verified the $1 / r$ profiles for an experimentally relevant case, $\tilde{r}_{i}=4$, We $=55$, using potential flow simulations using an axisymmetric Boundary Integral (BI) routine [15-18]. We solve the Laplace equation $\nabla^{2} \varphi=$ 0 for the flow potential $\varphi$ in the domain indicated in Fig. 8.3a, containing a small inlet region before the circular bend, ending with a separated sheet. In the simulations the contact line is pinned at a fixed position angle $\beta$. Note that the BI simulations are only used for the confirmation of the presumed velocity profile - we have not succeeded in creating perfectly steady sheets in the simulations, except for trivial solutions. 

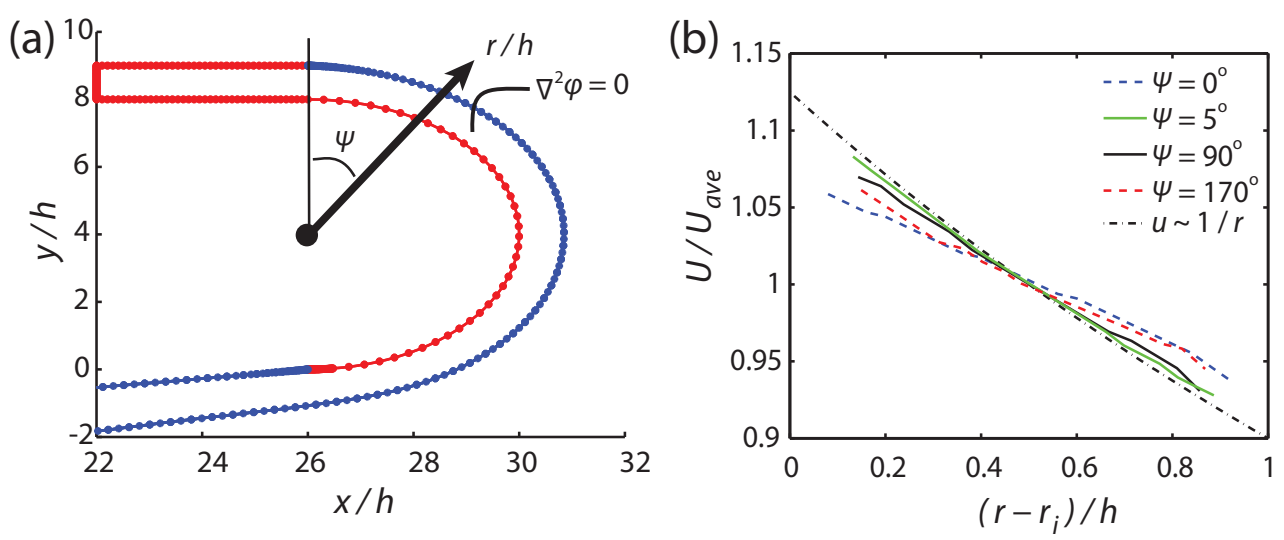

Figure 8.3: Potential flow simulation of the velocity profile in the flowing layer. (a) Sketch of the simulation domain. The red lines are undeformable solid boundaries, while blue edges are deformable and exhibit capillary pressure. The dots are nodes of the boundary integral simulations. Parameters are taken $\tilde{r}_{i}=4, \mathrm{We}=55$. The simulated setting is in fact axisymmetric, like the experiment using an impacter of radius $R_{i m p}[7]$; here we adapted a typical experimental value $R_{i m p} / h=26$. (b) Velocity profiles (normalized by the average $U_{\text {ave }}$ ), measured across the liquid film for different locations around the curved solid. The velocity profile quickly evolves towards the expected $1 / r$ profile (dash-dotted line). 
The result is shown in Fig. 8.3b, where we plot the normalized velocity profile across the film for various locations $\psi$ along the curved edge (defined in Fig. 8.3a). The initially uniform inflow indeed rapidly evolves towards a $1 / r$ profile, indicated as the dash-dotted line. The entrance/exit effects are quite small: for the example of Fig. 8.3 it is not more than a few degrees at the beginning of the bend, and about 10 degrees at the bottom part where the liquid separates from the solid. This shows that a $1 / r$ profile for $\tilde{r}_{i} \gg 1$ is a valid approximation.

Thus, we base our further calculations on the velocity profile $u=A / r$, were the constant $A$ is determined from mass conservation,

$$
U h=\int_{r_{i}}^{r_{i}+h} u d r=\int_{r_{i}}^{r_{i}+h} \frac{A}{r} d r,
$$

so that

$$
u=U\left(\frac{1}{\frac{r}{h} \ln \left(1+\frac{1}{\tilde{r}_{i}}\right)}\right) .
$$

The flow velocity directly above the solid surface is larger than at the top of the liquid sheet, so the pressure difference $P_{A}-P_{B}$ (cf. Fig. 8.2a) is indeed positive from the steady Bernoulli equation:

$$
\begin{aligned}
\Delta P & =P_{A}-P_{B}=\frac{1}{2} \rho\left(\left.u\right|_{r=r_{i}} ^{2}-\left.u\right|_{r=r_{i}+h} ^{2}\right) \\
& =\rho U^{2}\left[\frac{\frac{1}{2}+\tilde{r}_{i}}{\tilde{r}_{i}^{2}\left(1+\tilde{r}_{i}\right)^{2}\left(\ln \left(1+\frac{1}{\tilde{r}_{i}}\right)\right)^{2}}\right] .
\end{aligned}
$$

Computing $P_{A}$ (gauge) using the Young-Laplace pressure difference as

$$
P_{A}=\frac{\gamma}{h}\left[\frac{1}{1+\tilde{r}_{i}}\right],
$$

we obtain

$$
P_{B}=\frac{\gamma}{h}\left[\frac{1}{1+\tilde{r}_{i}}\right]-\rho U^{2}\left[\frac{\mathcal{G}\left(\tilde{r}_{i}\right)}{\tilde{r}_{i}}\right] .
$$

Here we introduced a dimensionless geometrical factor

$$
\mathcal{G}\left(\tilde{r}_{i}\right)=\frac{\frac{1}{2}+\tilde{r}_{i}}{\tilde{r}_{i}\left(1+\tilde{r}_{i}\right)^{2}\left(\ln \left(1+\frac{1}{\tilde{r}_{i}}\right)\right)^{2}},
$$


which encodes all relevant information of the velocity profile.

Using that $P_{B}=-\gamma / r_{m}$, we also extract an expression for $r_{m}$ :

$$
\frac{r_{m}}{h}=\frac{1+\tilde{r}_{i}}{\operatorname{We}\left(1+\frac{1}{\tilde{r}_{i}}\right) \mathcal{G}\left(\tilde{r}_{i}\right)-1} .
$$

Thus, the size of the meniscus $r_{m}$ adapts to accommodate the low pressure $P_{B}$. Note that We is typically large, and hence $r_{m} \ll r_{i}$.

\subsubsection{Calculating the forces}

We can now proceed to the evaluation of the several terms in the momentum balances $(8.1,8.2)$ by integrating the local pressures along the different sections of the CS of Fig. 8.2a, which is located just inside the liquid. Along the solid, we find for the force induced by the pressure difference projected in $\mathbf{x}$ direction, $F_{d, x}$ :

$$
\begin{aligned}
& F_{d, x}=\left[\int P_{B} d \mathcal{A}_{w e t}\right]_{x}=\int_{0}^{\beta} P_{B} r_{i} \sin \psi d \psi \\
& =\left[\rho U^{2} h \mathcal{G}\left(\tilde{r}_{i}\right)-\gamma\left(\frac{\tilde{r}_{i}}{1+\tilde{r}_{i}}\right)\right](\cos \beta-1),
\end{aligned}
$$

where the integration variable $\psi$ is the angle with respect to the vertical (see Fig. 8.3a). Similarly, we find for $F_{d, y}$ :

$$
\begin{aligned}
F_{d, y}= & {\left[\int P_{B} d \mathcal{A}_{w e t}\right]_{y}=-\int_{0}^{\beta} P_{B} r_{i} \cos \psi d \psi } \\
& =-\left[\rho U^{2} h \mathcal{G}\left(\tilde{r}_{i}\right)-\gamma\left(\frac{\tilde{r}_{i}}{1+\tilde{r}_{i}}\right)\right] \sin \beta .
\end{aligned}
$$

The capillary forces are induced by the Young-Laplace pressures over the free surface of the meniscus $\left(F_{m e n, x}\right.$ and $\left.F_{m e n, y}\right)$, and over the top free surface of the film $\left(F_{t o p, x}\right.$ and $\left.F_{t o p, y}\right)$. For the capillary force induced by the curvature of the top of the sheet, projected in the $\mathbf{x}$ direction, we find

$$
\begin{aligned}
F_{t o p, x} & =-\int_{0}^{\alpha} P_{A}\left(r_{i}+h\right) \sin \psi d \psi \\
& =-\int_{0}^{\alpha}\left(\frac{\gamma}{r_{i}+h}\right)\left(r_{i}+h\right) \sin \psi d \psi \\
& =\gamma[\cos \alpha-1],
\end{aligned}
$$


and

$$
F_{t o p, y}=-\gamma \sin \alpha .
$$

Note that the expressions for $F_{t o p, x}$ and $F_{t o p, y}$ are more easily interpreted by considering the explained equivalent picture of the force balance in Fig. 8.2b: the separate terms are the $\mathbf{x}$ and $\mathbf{y}$ projections of the capillary forces along the free surfaces of the CV. A similar observation holds for the capillary forces in the meniscus, which can be obtained by integrating the pressure $P_{B}$ over the meniscus circle with radius of curvature $r_{m}$

$$
\begin{aligned}
F_{m e n, x} & =\int_{-\left(\pi-\beta-\theta_{0}\right)}^{\alpha} P_{B} r_{m} \sin \psi d \psi \\
& =\int_{-\left(\pi-\beta-\theta_{0}\right)}^{\alpha}\left(-\frac{\gamma}{r_{m}}\right) r_{m} \sin \psi d \psi \\
& =-\gamma\left[\cos \left(-\left(\pi-\beta-\theta_{0}\right)\right)-\cos \alpha\right] \\
& =\gamma\left[\cos \left(\beta+\theta_{0}\right)+\cos \alpha\right],
\end{aligned}
$$

and

$$
F_{m e n, y}=\gamma\left[-\sin \left(\beta+\theta_{0}\right)-\sin \alpha\right] .
$$

Here we used the fact that the meniscus angle, $*$ in Fig. 8.2, is equal to $\pi-\theta_{0}-\beta+\alpha$, as follows from the geometry.

\subsubsection{Resulting system of equations}

The momentum balance $(8.1,8.2)$ combined with the computed forces finally yield the key equations of the model:

$$
\begin{aligned}
{\left[\mathrm{We}-\mathrm{We} \mathcal{G}\left(\tilde{r}_{i}\right)-\frac{1}{\left(1+\tilde{r}_{i}\right)}\right]+[2-\mathrm{We}] \cos \alpha } & \\
+\left[\mathrm{We} \mathcal{G}\left(\tilde{r}_{i}\right)-\frac{\tilde{r}_{i}}{\left(1+\tilde{r}_{i}\right)}\right] \cos \beta+\cos \left(\beta+\theta_{0}\right) & =0 \\
{[2-\mathrm{We}] \sin \alpha+\left[\mathrm{We} \mathcal{G}\left(\tilde{r}_{i}\right)-\frac{\tilde{r}_{i}}{\left(1+\tilde{r}_{i}\right)}\right] \sin \beta+\sin \left(\beta+\theta_{0}\right) } & =0
\end{aligned}
$$

with We $=\rho U^{2} h / \gamma$, as previously defined. This system should be seen as equations for the angles $\alpha$ and $\beta$, which can be solved for given values of We, 
$\tilde{r}_{i}$, and $\theta_{0}$. Note that by imposing a contact angle $\theta_{0}$, we by definition consider only separated sheets.

Before we proceed to the numerical solutions of $(8.16,8.17)$ in Sec. 8.3, we can already analyze a few interesting limits. The limit $\mathrm{We} \rightarrow \infty$ gives as only solution $\alpha=\beta=0$, corresponding to the perfect horizontal sheet, for every value of $\theta_{0}$. Interestingly, the same holds for $\theta_{0}=180^{\circ}$, for which there is no capillary adhesion: if the surface is superhydrophobic, the jet/sheet will be perfectly horizontal for any We. A third interesting case is $\tilde{r}_{i} \rightarrow \infty$, for which $\mathcal{G}\left(\tilde{r}_{i}\right) \rightarrow 1$ and no separated solution exists, unless $\theta_{0}=180^{\circ}$. These trends all agree with the experiments in Ref. [7]. A final special case is $\mathrm{We}=2$. In that case, the $\alpha$ dependence completely drops out of the equations, and we are left with two equations for a single unknown, $\beta$. This has no solution unless $\theta_{0}=180^{\circ}$. We can interpret $\mathrm{We}=2$ as a minimum flow speed needed for a non-retracting sheet, as this indeed coincides with the Taylor-Culick velocity $[19,20]$.

\subsection{Results}

\subsubsection{Solutions}

We now analyze the solutions of the momentum balance $(8.16,8.17)$. In Fig. 8.4a we report the separation angle $\alpha$ as a function of We for $\tilde{r}_{i}=4$. The various curves correspond to different wettabilities, with $\theta_{0}$ increasing along the arrow. It is found that solutions only exist above a critical value of the Weber number, $\mathrm{We}_{c}$, which we identify as the threshold for the trickling transition. The critical point is found to coincide with $\alpha=180^{\circ}$. Above $\mathrm{We}_{c}$, the momentum balance admits two possible solutions. However, solutions for $\alpha$ larger than $180^{\circ}$ are not physical in the sense that the liquid would cross the solid and we focus on the lower solution branch. As expected, the deflection angle $\alpha$ increases when the fluid's inertia is reduced, i.e. as the Weber number is decreased. The sketches in Fig. 8.4b-d further illustrate this effect. It should be emphasized that the deflection angle varies rapidly with We for $\alpha$ beyond $90^{\circ}$, i.e. when the critical point is approached. The inset of Fig. 8.4a shows a zoom around the critical point for both angles, $\alpha$ (blue solid line) and $\beta$ (red dashed line) for $\theta_{0}=90^{\circ}$. The two angles always take similar values, with a maximum difference of about $20^{\circ}$. The global minimum of $\beta$ is also reached at $\mathrm{We}_{c}$, but has a value slightly below $180^{\circ}$ (equal to $180^{\circ}$ if $\theta_{0}=0^{\circ}$ ).

The critical Weber number is thus found to depend on the wettability of the solid. Figure 8.5 presents $\mathrm{We}_{c}$ as a function $1+\cos \theta_{0}$ for several values 


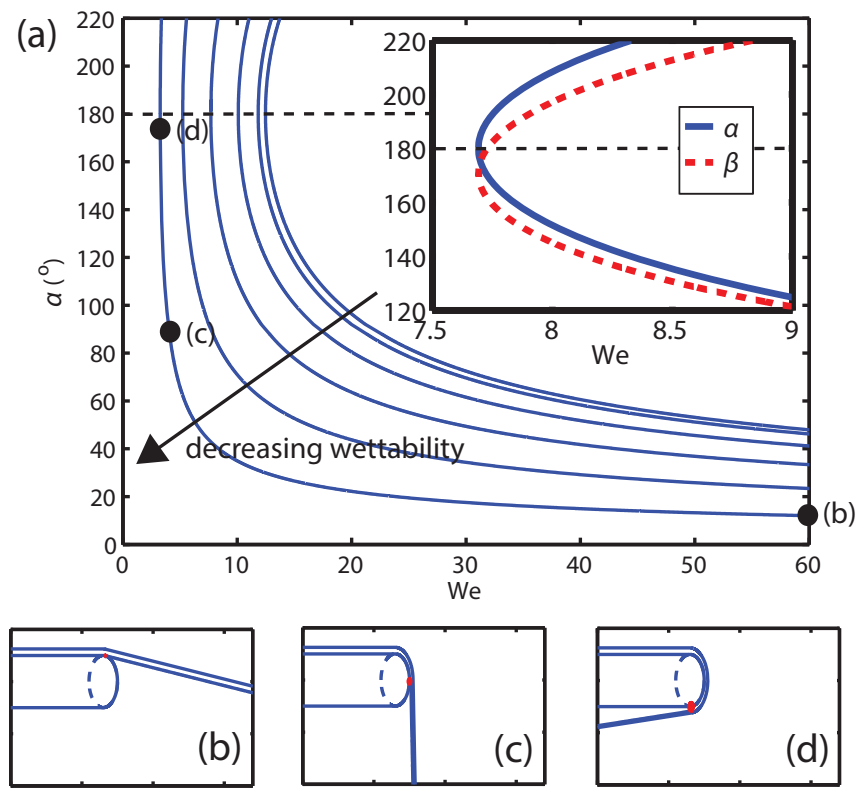

Figure 8.4: (a) $\alpha$ vs $\mathrm{We}=\rho U^{2} h / \gamma$ for $\tilde{r}_{i}=4$ and different contact angles. Following the arrow, $\theta_{0}$ increase from $0^{\circ}$ to $150^{\circ}$ in steps of $30^{\circ}$. Solutions only exist for We larger than a critical Weber number $\mathrm{We}_{c}$. Inset: a detailed view of $\alpha$ and $\beta$ as a function of We around $\mathrm{We}_{c}$ for $\theta_{0}=90^{\circ}$. (b-d) Resulting flow contours, corresponding to the marked dots in panel (a), showing the dependence of the separation angle on the Weber number for $\theta_{0}=150^{\circ}$. 


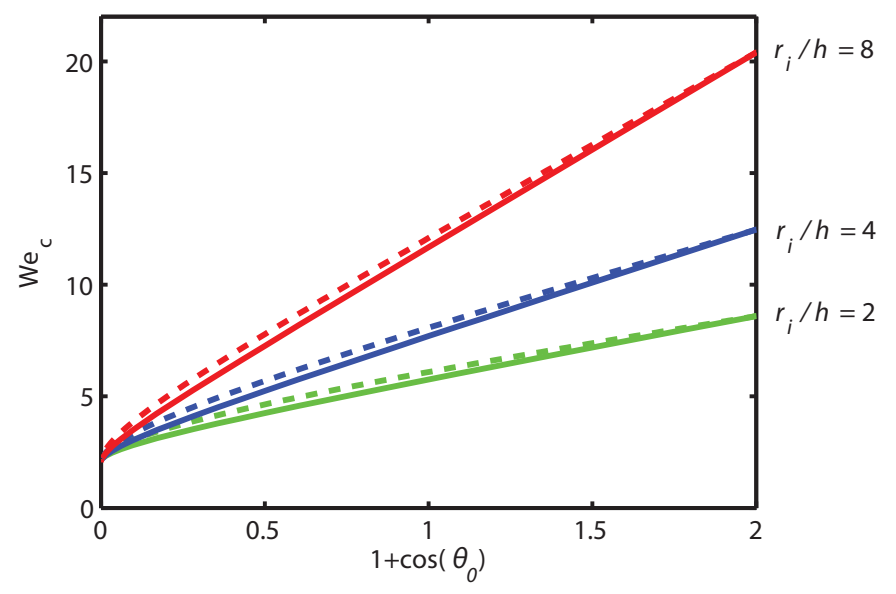

Figure 8.5: Critical Weber number $\mathrm{We}_{c}$ as a function of $1+\cos \theta_{0}$ for $r_{i}=$ $0.5,1$, and $2 \mathrm{~mm}$, and $h=0.25 \mathrm{~mm}$. Solid lines are numerical solutions of the momentum balance; dashed lines correspond to the approximation (8.19).

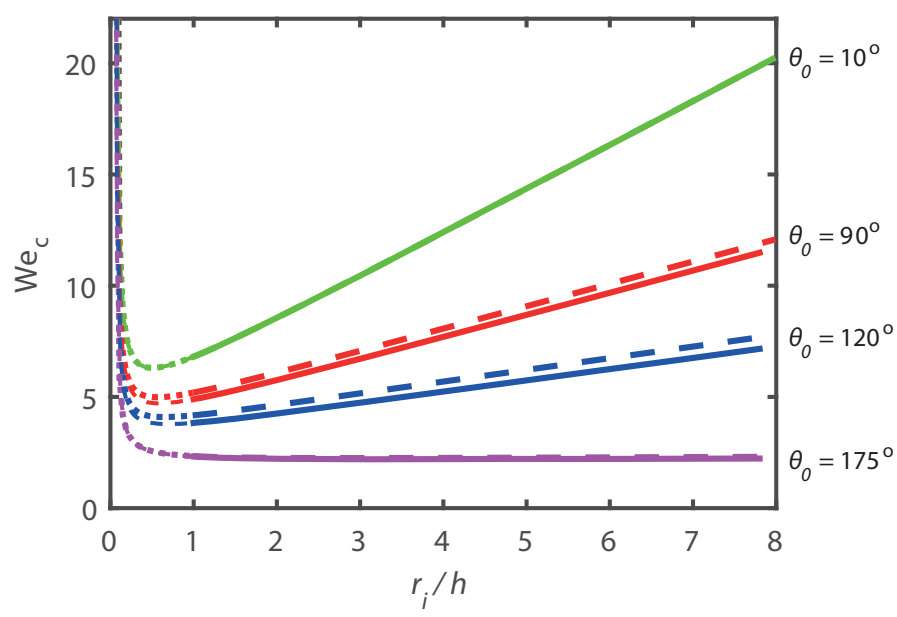

Figure 8.6: Critical Weber number $\mathrm{We}_{c}$ as a function of $\tilde{r}_{i}=r_{i} / h, h=$ $0.25 \mathrm{~mm}$, for $\theta_{0}=10^{\circ}, 90^{\circ}, 120^{\circ}$, and $175^{\circ}$. Solid lines are numerical solutions of the momentum balance; dashed lines correspond to the approximation (8.19). The model is not valid below $\tilde{r}_{i} \sim 1$, where the predictions are given by a dotted line. 
of $\tilde{r}_{i}$ (solid lines). The dependence is approximately linear, with a slope that increases for increasing $\tilde{r}_{i}$. The linearity no longer applies when $1+\cos \theta_{0}$ is small, i.e. the superhydrophobic limit, for which all curves converge to $\mathrm{We}_{c}=2$. In this case there is no adhesion by the solid and the minimal velocity is given by the Taylor-Culick velocity of a free sheet.

Figure 8.6 shows $\mathrm{We}_{c}$ as a function of the radius of curvature of the solid $\tilde{r}_{i}$, for several values of $\theta_{0}$ (solid lines). For large $\tilde{r}_{i}$ we again find a linear trend, reflecting that it is more difficult to separate the sheet when the edge of the solid is not sharp. Note that the model is only valid for $\tilde{r}_{i} \geq 1$, owing to the assumptions of the velocity field that leads to (8.8). It is still interesting to report the model prediction for $\tilde{r}_{i}<1$ : there is even a divergence that appears when $\mathcal{G}$ takes the value 1 (Fig. 8.6, dotted lines). In the Discussion section we briefly comment on the limit of small $\tilde{r}_{i}$.

\subsubsection{Asymptotic expansion}

It is instructive to attempt an approximate solution for the critical Weber number, based on the observation that at the critical point $\alpha=180^{\circ}$ and $\beta=180^{\circ}-\epsilon$, where $\epsilon$ turns out to be small $\left(<20^{\circ}\right)$. Hence, we expand Sys. $(8.16,8.17)$ around up to first order in $\epsilon$, replacing $\sin \epsilon \sim \epsilon$ and $\cos \epsilon \sim 1$. Using $\sin \left(\theta_{0}-\epsilon\right)=\sin \theta_{0} \cos \epsilon-\cos \theta_{0} \sin \epsilon$ and $\cos \left(\theta_{0}-\epsilon\right)=\cos \theta_{0} \cos \epsilon-$ $\sin \theta_{0} \sin \epsilon$, we find for $\epsilon$ (in radians):

$$
\epsilon \approx \frac{\sin \theta_{0}}{\mathcal{G}\left(\tilde{r}_{i}\right) \mathrm{We}+\cos \theta_{0}-\frac{\tilde{r}_{i}}{1+\tilde{r}_{i}},}
$$

and

$$
\mathrm{We}_{c}^{2}+A \mathrm{We}_{c}+B \approx 0,
$$

with

$$
A=\frac{2\left(\frac{\tilde{r}_{i}}{1+\tilde{r}_{i}}\right)+\cos \theta_{0}\left(3 \mathcal{G}\left(\tilde{r}_{i}\right)-2\right)+2 \mathcal{G}\left(\tilde{r}_{i}\right)\left(1-\frac{\tilde{r}_{i}}{1+\tilde{r}_{i}}\right)+\mathcal{G}\left(\tilde{r}_{i}\right)\left(\frac{1-\tilde{r}_{i}}{1+\tilde{r}_{i}}\right)}{-2 \mathcal{G}\left(\tilde{r}_{i}\right)+2 \mathcal{G}\left(\tilde{r}_{i}\right)^{2}}
$$

and

$$
B=\frac{-2\left(\frac{\tilde{r}_{i}}{1+\tilde{r}_{i}}\right)+2 \cos \theta_{0}+\frac{\tilde{r}_{i}\left(\tilde{r}_{i}-1\right)}{\left(1+\tilde{r}_{i}\right)^{2}}-\left(\frac{2 \tilde{r}_{i}-1}{1+\tilde{r}_{i}}\right) \cos \theta_{0}+1}{-2 \mathcal{G}\left(\tilde{r}_{i}\right)+2 \mathcal{G}\left(\tilde{r}_{i}\right)^{2}} .
$$


This gives a quadratic equation for $\mathrm{We}_{c}$, which is superimposed as dashed lines in Figs. 8.5 and 8.6. Indeed, this approximate solution gives a very good description of the full solutions. The difference is largest for $\theta_{0} \sim 90^{\circ}$, where the largest values for $\epsilon$ are encountered.

We could even further simplify (8.19) when $\tilde{r}_{i} \gg 1 . \mathcal{G}\left(\tilde{r}_{i}\right)$ can then be approximated by $\left.1-1 /\left(2 \tilde{r}_{i}\right)\right)$, and this yields

$$
\mathrm{We}_{c} \sim \tilde{r}_{i}\left(1+\cos \theta_{0}\right) .
$$

This predicts linear behavior of the critical Weber number with respect to both the aspect ratio $\tilde{r}_{i}$ and to $1+\cos \theta_{0}$, which is consistent with our results in Figs. 8.5 and 8.6.

\subsection{Discussion}

We have performed a control volume analysis of inertial pouring flows, taking into account the fluid inertia, surface tension, and the curvature of the edge of the solid. The analysis explicitly recovers that steady states can only exist above a critical Weber number $\mathrm{We}_{c}$, and thus captures the transition to trickling. The work also recovers the experimental trends that $\mathrm{We}_{c}$ decreases as the solid is sharper and more hydrophobic, and identify a lower bound $\mathrm{We}_{c} \geq 2$. Here we wish to conclude by making a direct comparison to experiments $[7,8]$.

Figure 8.7a reports the separation angle $\alpha$ against $\mathrm{We} / \mathrm{We}_{c}$ for three different contact angles, $\theta_{0}=10^{\circ}, 115^{\circ}$, and $175^{\circ}$. The value of $\tilde{r}_{i}=4$ was adapted from the experiment, and the symbols represent data from Ref. [7]. It is clear that the model captures the experimental trends, but is limited in terms of quantitative prediction. In particular, the change of angle $\alpha$ close to the critical point is underpredicted by the model. Also, the experiments at large Weber number exhibit a sheet deflection of the order of $10^{\circ}$, which points to either dissipation in the fluid or an influence of the axisymmetric setup - both of which are not taken into account in the model.

In Fig. 8.7b, we show the critical Weber number $\mathrm{We}_{c}$ as a function of $1+\cos \theta$, for three different $\tilde{r}_{i}$. The model nicely captures the linear dependence $\mathrm{We}_{c} \sim\left(1+\cos \theta_{0}\right)$ that is observed in experiment. In addition, the data in Fig. $8.7 \mathrm{~b}$ are consistent with the predicted lower bound on the critical Weber number of $\mathrm{We}_{c}=2$, set by the Taylor-Culick velocity. The model underestimates the dependence on $\tilde{r}_{i}$ : we predict a linear increase of $\mathrm{We}_{c}$ with $\tilde{r}_{i}$, while the experimental data are better described by $\tilde{r}_{i}^{2}$, as was also proposed by a scaling argument [7]. However, the recent data in Ref. [8] are 
(a)

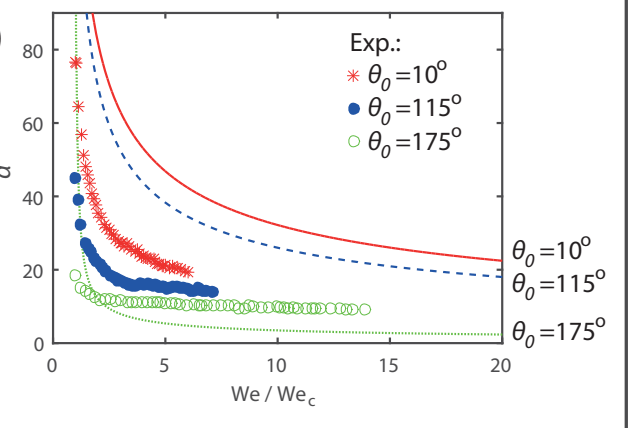

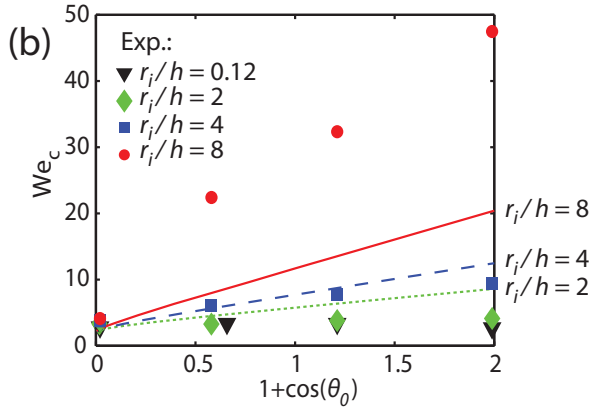

Figure 8.7: Quantitative comparison between our model and experimental data taken from Duez et al. [7]. (a) $\alpha$ vs. We $/ \mathrm{We}_{c}$ for $\theta_{0}=10^{\circ}, 115^{\circ}$, and $175^{\circ} ; r_{i}=1 \mathrm{~mm}$ and $h=0.25 \mathrm{~mm}$; (b) We $\mathrm{Ws}_{c}$. $\left(1+\cos \theta_{0}\right)$ for several $\tilde{r}_{i}=r_{i} / h$.

best described by a linear dependence on $\tilde{r}_{i}$, even though their experimental setup is in principle comparable. At present, there is thus some uncertainty on how the sharpness of the edge affects the trickling transition. We do note that the model predictions shown here are without adjustable parameters and the discrepancy with, and between, various experiments is always less than a factor of two.

The momentum balance presented in this chapter has the merit that it provides a general framework for future investigations of pouring flows. Namely, the capillary forces are described correctly here - apart perhaps from the question of whether $\theta_{0}$ can be interpreted as the equilibrium contact angle [21, 22] - and the main assumption is on the estimate of the hydrodynamic force $F_{d}$. As mentioned already, we only estimated this force in the case of large $\tilde{r}_{i}$, but $(8.1,8.2)$ can in fact be used to estimate $F_{d}$ based on experimental data. The black triangles in Fig. 8.7b correspond to experiments with $\tilde{r}_{i}=0.12$, for which $\mathrm{We}_{c}$ exhibits almost no dependence on wettability and is close to the lower bound $\mathrm{We}_{c}=2$. The momentum balance suggests that $F_{d} \rightarrow 0$ in this limit, implying a vanishing hydrodynamic retention around sharp edges. Interestingly, this is a strong departure from the exact potential flow solutions around a perfectly sharp edge [3, 4], which do not include capillary adhesion, for which trickling completely relies on a nonzero $F_{d}$. Future work should further reveal how the presence of a meniscus on a sharp edge influences trickling in inertial pouring flows. 


\section{References}

[1] M. Reiner, "The teapot effect... a problem", Phys. Today 9, 16 (1956).

[2] J. B. Keller, "Teapot effect", J. App. Phys. 28, 859-864 (1957).

[3] J.-M. Vanden-Broeck and J. B. Keller, "Pouring flows", Phys. Fluids 29, 3958-3961 (1986).

[4] J.-M. Vanden-Broeck and J. B. Keller, "Pouring flows with separation", Phys. Fluids A 1, 156-158 (1989).

[5] B.-S. Yoon and Y. A. Semenov, "Separated inviscid sheet flows", J. Fluid Mech. 678, 511-534 (2011).

[6] S. F. Kistler and L. E. Scriven, "The teapot effect: sheet-forming flows with deflection, wetting and hysteresis", J. Fluid Mech. 263, 19-62 (1994).

[7] C. Duez, C. Ybert, C. Clanet, and L. Bocquet, "Wetting controls separation of inertial flows from solid surfaces", Phys. Rev. Lett. 104, 084503 (2010).

[8] Z. Dong, L. Wu, J. Wang, J. Ma, and L. Jiang, "Superwettability controlled overflow", Adv. Mater. 27(10), 1745-1750 (2015).

[9] C. Clanet, "Waterbells and liquid sheets", Annu. Rev. Fluid Mech. 39, 469-496 (2007).

[10] H. Lhuissier and E. Villermaux, "Crumpled water bells", J. Fluid Mech. 693, 508-540 (2012).

[11] E. Dressaire, L. Courbin, A. Delancy, M. Roper, and H. A. Stone, "Study of polygonal water bells: inertia-dominated thin-film flows over microtextured surfaces", J. Fluid Mech. 721, 46-57 (2013).

[12] F. L. Orr, L. E. Scriven, and A. P. Rivas, "Pendular rings between solids: meniscus properties and capillary force", J. Fluid Mech. 67, 723-742 (1975).

[13] P. A. Kralchevsky and K. Nagayama, Particles at fluid interfaces and membranes, Elsevier, 469-502 (2001).

[14] E. Guyon, J. P. Hulin, L. Petit, and C. D. Mitescu, Physical Hydrodynamics, Oxford University Press, New York (2001). 
[15] H. N. Oguz and A. Prosperetti, "Dynamics of bubble growth and detachment from a needle", J. Fluid Mech. 257, 111-145 (1993).

[16] R. P. H. M. Bergmann, D. van der Meer, S. Gekle, J. van der Bos, and D. Lohse, "Controlled impact of a disk on a water surface: Cavity dynamics", J. Fluid Mech. 633, 381-409 (2009).

[17] S. Gekle and J. M. Gordillo, "Compressible air flow through a collapsing liquid cavity", Int. J. Numer. Meth. Fluids 67, 1456-1469 (2011).

[18] W. Bouwhuis, K. G. Winkels, I. R. Peters, P. Brunet, D. van der Meer, and J. H. Snoeijer, "Oscillating and star-shaped drops levitated by an airflow", Phys. Rev. E 88, 023017 (2013),

See Chapter 6 of this thesis.

[19] G. I. Taylor, "The dynamics of thin sheets of fluid. III. disintegration of fluid sheets", Proc. R. Soc. A 253, 313-321 (1959).

[20] F. E. C. Culick, "Comments on a ruptured soap film", J. Appl. Phys. 31(6), 1128-1129 (1960).

[21] D. Bonn, J. Eggers, J. Indekeu, J. Meunier, E. Rolley, "Wetting and spreading", Rev. Mod. Phys. 81(2), 739-805 (2009).

[22] J. H. Snoeijer, B. Andreotti, "Moving contact lines: scales, regimes and dynamical transitions", Ann. Rev. Fluid Mech. 45, 269-292 (2013). 


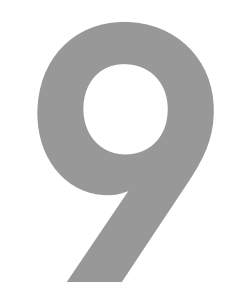

\section{Summary and outlook}

\subsection{Summary}

\subsubsection{Small air bubble entrapment at liquid impact}

In Chapters 2-4 we focus on small air bubble entrapment during the impact of a liquid drop on a solid surface, a liquid drop on a liquid pool, and a solid sphere on a liquid pool. There are a few different mechanisms for air bubble entrapment that appear for these impact scenarios, and in the first chapters of this thesis we study in detail the small scale deformations of the liquid surface(s) at the bottom of the drop/object, induced by the force exerted by the gas that is squeezed out between the falling object and the bottom surface. In Chapter 2 we start with impacting drops onto solid surfaces. By performing interferometry experiments, we are able to visualize the micrometer scale deformations of the drop, just before the drop touches and wets the surface. The results of the experiments and Boundary Integral simulation confirm that there exists a maximal entrained bubble size for some impact velocity (keeping the drop size, and, of course, the liquid and gas properties constant). In the simulations we assume that the liquid within the drop obeys potential flow, and the air in the small gas layer obeys viscous lubrication flow. We explain the maximal bubble entrapment as follows: for large drops and/or large impact velocities, it requires a large force to locally slow down and deform the drop - 
the entrained air bubble will be small. However, for small drops and/or small drop velocities, the surface tension of the liquid acts against the deformation of the drop, also minimizing the size of the air bubble. In between these two regimes, there is a cross-over, where we find maximal air bubble volumes. We estimate this maximum from scaling theory, the interferometry experiments, and the Boundary Integral simulations.

Once we resolved the mechanism for small air bubble entrapment for a drop impacting onto a solid surface, the natural question arises whether the same applies to the other impact scenarios: a sphere onto a pool, and a drop onto a pool. This is the central question addressed in Chapter 3. Using Boundary Integral simulations and a collection of experimental data from the literature, we indeed find that the mechanism explained above is universal and that the sizes of the entrained air bubbles are in fact equal for the three impact scenarios, except for the fact that the bubbles at impact of a liquid drop onto a liquid pool are larger by a factor of exactly 2 . The equivalence between the scenarios can be explained from the fact that the deformations are very localized, on a length-scale much smaller than the size of the drop/sphere, yielding a symmetry between the several cases. The factor 2 in case of liquid drop impact on a pool can be explained by noting that in that case the air pressure deforms two liquid surfaces instead of one.

In Chapters 2 and 3 we have been particularly interested in the final deformation of the involved liquid surfaces, up to the moment of touch-down. Namely, this determines the size of the entrapped air bubble. In Chapter 4 we take a step back and apply a perturbation expansion to find out how the ini- $^{-}$ tial deformations evolve over time and converge to the resulting dimple shape just before touch-down. This is of particular interest, since the scaling laws in Chapters 2 and 3 are based on the initial stages. It turns out that we need to distinguish between inertial gas flow and viscous gas flow, and large-gap gas flow and thin-gap gas flow. The two cross-overs are quantified in detail and scaling laws for the regimes are presented. For 'typical' impact parameters (a millimeter sized drop falling at its terminal velocity of a few meters per second), the falling drop/object first encounters a large-gap inertial regime, followed by a thin-gap inertial regime, and finally a thin-gap viscous flow regime. For all cases we derive the spatio-temporal evolution of the pool surface, and, surprisingly, our analysis reveals that inertial and viscous cushioning both lead to a pool deflection of the order of a micrometer. However, we still find that the bubble volume is mainly determined by the effect of gas viscosity, as the Stokes gas pressure is highly localized and most strongly increases during the 
final stages. This explains the very good agreement we found in Chapters 2 and 3 between the experiments and the numerical simulations, where in the latter case we neglect the inertia of the gas.

\subsubsection{High-speed microdrop train impact on a pool}

In Chapter 5 we consider the impact of a high-speed train of micrometer sized drops onto a deep liquid pool. The momentum of the successive impacting drops is large enough to create a deep and narrow cavity, with a depth of about one thousand times the size of the individual drops. The cavity shape and its maximal depth primarily depend on a balance between inertia and surface tension; due to the small size of the drops, hydrostatics only has a relevant influence if the cavity grows deep enough. The collapse of the cavity is thus fully determined by capillary effects, and typically occurs at the top, near the pool surface. We identify the scaling laws that characterize the shape, time to pinch-off and maximal depth of the cavity.

\subsubsection{Levitated drops}

In Chapter 6 we make the step from impacting drops to levitated drops, i.e., drops which are completely lifted from a surface as a result of a continuous gas flow at the drop bottom. This situation arises for example for Leidenfrost drops, where the gas on which the drop floats comes from the drop, which is evaporating, itself. Leidenfrost drops can spontaneously start to oscillate and break symmetry, and the mechanism of this behavior, called the star-drop instability, has not yet been resolved. By injecting the gas flow beneath the drop, we can make the drop float on an external gas layer, at room temperature. In these experiments, we observed the very same oscillating and symmetry-breaking behavior compared to the Leidenfrost case, ruling out the influence of temperature and heat transfer on the star-drop instability. The oscillations thus must have a hydrodynamic origin, and in Chapter 6 we indeed reproduce the instability using (hydrodynamic) Boundary Integral simulations. The simulations also reproduce steady levitated drops and gas pockets breaking through the drops (the 'chimney' instability). We find that the oscillatory instability consists of two stages: an axisymmetric stage, which is an interplay between the liquid flow and the gas flow, and a symmetrybreaking stage, where the oscillations make the drop break symmetry with the preferred oscillation mode (the Rayleigh mode). 


\subsubsection{Drop shaping by laser-pulse impact}

In Chapter 7 we study the response of liquid drops impacted by a focused laser pulse. This is motivated by Extreme Ultraviolet (EUV) lithography, which is used for the creation of nanometer-sized patterns on electronic chips. The role of technology in our society keeps growing at an impressively high rate. To print the small features at high resolution, one needs small wavelength radiation. In EUV-machines this radiation results from liquid tin sheets that are impacted by a laser-pulse, resulting in the formation of plasma. The sheets are created by drops impacted by a first laser-pulse. The strong local evaporation due to the impact of the laser results in a recoil of the drop: the drop propels and expands to a sheet till fragmentation occurs. The fluid dynamics play a crucial role for improving the efficiency of the EUV machine. We perform an experimental analysis and Boundary Integral simulations, in which in the latter case the energy input is modeled by a very short pressure pulse acting along the drop surface. We find that the propulsion speed and the maximal radius of the expansion are proportional to the laser pulse energy and provide scaling laws for the dependency of these parameters on the shape of the pulse. The expansion dynamics are limited by surface tension, in a way similar to the limitation of the spreading of a drop impacting on a solid surface.

\subsubsection{The effect of wettability on inertial pouring flows}

Chapter 8 describes a study of 'inertial pouring flows', fast flowing liquids separating from a curved solid surface. A typical daily life example of a pouring flow is the flow of tea from the spout of a teapot. If the pouring velocity is too small, the liquid does not separate from the solid, but trickles down the spout, a phenomenon which is called 'the teapot effect', and which annoys a lot of people. While a former study has shown that the angle of separation depends on the wettability of the solid in an unexpected degree (and that the teapot effect can even be completely beaten by using a super hydrophobic teapot spout), a physical explanation for the trickling behavior was not yet found. By solving the 2D linear momentum balance, taking into account the flow profile and the capillary forces on the top and bottom of the liquid, we analytically find that, indeed, no steady (stable) solutions exist for small flow velocities, which leads to trickling. We quantify this minimum flow velocity as a function of the flow parameters, and recover the main experimental trends. 


\subsection{Outlook}

To conclude this thesis, we now discuss some possible directions for future work.

\subsubsection{Small air bubble entrapment at liquid impact}

The maximal volume of a bubble entrapped during drop impact onto a solid surface is observed in both the experimental and Boundary Integral simulation results and explained by scaling theory. However, if one looks more critically to Fig. 2.2, two things catch the eye, which are left unaddressed in Chapter 2.

Firstly, the numerical results show that the maximum of the bubble volume is located at a smaller Stokes number than the maximum of the dimple height; the maximum in Fig. 2.2b is shifted somewhat to the left with respect to the maximum in Fig. 2.2a. Figure 2.5a shows how this can be possible: the numerical dimple profiles in the cross-over regime turn out to be typical 'double dimples': the profiles exhibit a kink. In the Boundary Integral simulations, it seems that, within the cross-over regime, the dimple profile is a superposition of the narrow profile induced by the inertial behavior of the liquid, and the typically broader (and lower) profile induced by capillary effects. In the crossover both play a role. It would be worth investigating these features in more detail.

Secondly, the scaling prediction of the bubble volume in the capillary regime does not agree quantitatively with the experimental and numerical results. These results seem to suggest $V_{b} \sim R^{3} \mathrm{Ca}^{2}$, instead of our scaling prediction of $V_{b} \sim R^{3} \mathrm{Ca}$. In the capillary regime, where the dimple formation process happens relatively slow, the air has much more time to 'drain out' after the central height of the drop bottom converged to $H_{d}$. This would indeed decrease the bubble volume with respect to our scaling predictions. Recently, it has been found that draining of the air possibly even changes the bubble volumes in the inertial regime [1]. We can also imagine that, for example, the wettability and roughness of a solid surface plays a crucial role during the formation of a bubble from an air film [2]. The touch-down between the two surfaces might imply the break down of our assumption of macroscopic hydrodynamics, and one should start to take into account the mean free path of the moving gas. A possible suggestion is to study the first contact using Molecular Dynamics (MD) simulations, which might give interesting clues.

Besides the touch-down and rupture mechanisms, one could think of an other kind of liquid-solid interaction at liquid impact, but before the moment of 
touch-down. Ongoing research focuses on drop impact on structured surfaces, pillars (with diameters with the order of magnitude of the size of the impacting drop), or on several - non-symmetric - shapes of the solid surface. Indeed, the assumption of axisymmetry in our simulations is also a limitation on the possible geometries. To account for 3D impact problems [3], the modeling technique should be extended to 3D. With a 3D model also oblique collisions can be investigated.

\subsubsection{High-speed microdrop train impact on a pool}

In our study of drop train impact, we observe that neglecting the airflow in the numerical simulations causes significant deviation between the experiments and the simulations on the width of the cavity (not on the depth). The falling drops induce an airflow within the cavity, slowly sucking inwards the cavity walls, which also reduces the collapse time. For the large cavities created by the impact of a solid cylinder on a pool [4], where the cavity collapse is dominated by hydrostatics, it turned out that the airflow only influences the liquid dynamics during the very final stages of the collapse, which is thus a significant difference with respect to the case of a microdrop train. We attribute the more relevant influence of the air to the higher impact velocity and smaller length scale of our problem. Simulating the airflow would thus be an interesting step in the simulations. One could think of performing twophase Boundary Integral simulations, comparable to what has been done for the impact of a solid cylinder on a pool, where the gas has been treated as an inertial fluid, possibly treating the drops as falling solid masses.

Furthermore, we observe that the collapse of the cavity primarily depends on capillary effects. This is specific to microsized drops, and very different from the more commonly studied mm-sized drops, for which gravity is important. However, the size of the drops is not the only parameter of interest. When we look at the impact of finite amounts of liquid volume, the timescale of the total impact is also expected to be highly relevant for the cavity behavior. The cavities induced by impacting small drops with high frequency or velocity is expected to be completely different from the cavities resulting from the impact of large drops with low frequency or velocity. The classification of impact phenomena of finite amounts of liquid volume would be an interesting new aspect with respect to both the work on large water masses by, for example, Oguz, Prosperetti \& Kolaini [5], and our work presented in Chapter 5. 


\subsubsection{Levitated drops}

Our experiments and numerical simulations on levitated drops at room temperature have demonstrated that the star-drop instability is induced by a hydrodynamic mechanism, but we still lack a detailed description of the origin of the instability. It is now obvious that the instability is a result from the interplay between the liquid flow and the gas flow, but why does the instability not occur for small drops and/or small flow rates? In our simulations, we found that the threshold depends strongly on the density of the liquid, the viscosity of the gas, gravity, the surface tension and the damping constant ('artificial liquid viscosity') - together with the drop size and the airflow velocity these are basically all the parameters involved in the simulations. In the experiments, the location of the threshold does not depend on the liquid viscosity (contrarily to the simulations), but the drop dynamics are strongly dependent on the viscosity. In case of a large liquid viscosity, only the axisymmetric ('breathing') modes are observed; for small viscosities, the drops break symmetry and we observe Rayleigh oscillations. Studying the influence of liquid viscosity on the drop dynamics might be the most interesting next step, because for simulations of pure viscous drops on viscous airflows (i.e., assuming Stokes flow for both the drop and the gas) no oscillations were found [6]. In the simulations, this would need for an alternative simulation method (dropping the assumption of potential flow behavior). Furthermore, this is a second example of a flow geometry where it would be very interesting to drop the assumption of axisymmetry, such that it is possible to simulate how the original axisymmetric interplay between the liquid flow and gas flow leads to a spontaneous symmetry breaking of the drop.

\subsubsection{Drop shaping by laser-pulse impact}

Since the fluid dynamics are crucial for the improvement of the efficiency of EUV lithography machines, quantifying the influence of the impact of a laserpulse onto a liquid drop is important future work within this subject. This includes new modeling techniques, experiments, and simulations. Obviously, our Boundary Integral method does not capture phase transitions (vaporization), which is why alternative simulation methods (Lattice-Boltzmann) will also be applied to study the interaction of the liquid with the laser. Another limitation of our method is fragmentation and, by this, symmetry breaking. In Chapter 7 we particularly study the dependence of the drop translation and deformation, but another interesting dependency is the shape of the impacting 
laser-pulse, or, in more technical terms, the focal distance of the laser with respect to the drop position. In our Boundary Integral simulations, besides the amplitude and duration of the pressure pulse, we can also directly vary the width of the pulse, referring to a varying focal distance of the laser. To understand the influence of the pulse shape on the drop in detail, more simulations and experiments are needed.

\section{References}

[1] E. Klaseboer, R. Manica, and D. Y. C. Chan, "Universal behavior of the initial stage of drop impact", Phys. Rev. Lett. 113, 194501 (2014).

[2] J. San Lee, B. M. Weon, J. H. Je, and K. Fezzaa, "How does an air film evolve into a bubble during drop impact?", Phys. Rev. Lett. 109, 204501 (2012).

[3] R. C. A. van der Veen, M. H. W. Hendrix, T. Tran, C. Sun, P. A. Tsai, and D. Lohse, "How microstructures affect air film dynamics prior to drop impact", Soft Matter 10(21), 3703-3707 (2014).

[4] S. Gekle, I. R. Peters, J. M. Gordillo, D. van der Meer, and D. Lohse, "Supersonic air flow due to solid-liquid impact", Phys. Rev. Lett. 104, 024501 (2010).

[5] H. N. Oguz, A. Prosperetti, and A. R. Kolaini, "Air entrapment by a falling water mass", J. Fluid Mech. 294, 181-207 (1995).

[6] J. H. Snoeijer, P. Brunet, and J. Eggers, "Maximum size of drops levitated by an air cushion", Phys. Rev. E 79, 036307 (2009). 


\section{Samenvatting (Nederlands)}

Vallende druppels of druppels zwevend op een stromend gas (lucht of damp), kunnen allerhande krachten ervaren, die de druppels van vorm doen veranderen ten opzichte van hun evenwichtsvorm, een perfecte bol. Deze vervormingen zijn een belangrijk onderzoeksgebied binnen de vloeistoffysica, omdat ze de landing van een druppel op een oppervlak, de vorming van een vloeistofsheet, en/of het opbreken in kleinere druppels significant beïnvloeden. Inslag van een vloeistof en/of $o p$ een vloeistof is alomtegenwoordig in de industrie en de landbouw.

In Hoofdstukken 2-4 onderzoeken we kleine luchtbellen die kunnen worden ingevangen tijdens de inslag van een vloeistofdruppel op een solide oppervlak, een vloeistofdruppel op een vloeistofbad, en een solide bol op een vloeistofbad. Voor invanging van een luchtbel bij deze inslagscenario's bestaan verschillende mechanismen. In de eerste hoofdstukken van dit proefschrift lossen we de kleine-schaal vervorming van het vloeistofoppervlak aan de bodem van het vallende object, als resultaat van de kracht uitgeoefend door de lucht die uit de ruimte tussen het vallende object en het onderoppervlak wordt geperst, in detail op. In Hoofdstuk 2 beginnen we met de inslag van een druppel op een solide oppervlak. Door middel van interferometrie-experimenten is het mogelijk om de vervormingen van de orde van een paar micrometer, net voordat de druppel het oppervlak raakt, in beeld te brengen. De hoogte en breedte van de vervorming en het volume van de ingevangen luchtbel worden gemeten voor variërende inslagsnelheden. Zogeheten 'Boundary Integral' computersimulaties laten samen met de experimenten zien dat er een maximaal belvolume bestaat voor een zekere inslagsnelheid (voor constante druppelgrootte en constante eigenschappen van de vloeistof en de lucht). We verklaren het gevonden maximum in het belvolume als volgt: voor grote druppels en/of grote inslagsnelheden vergt het een grote tegenwerkende kracht om de druppel lokaal af te remmen en te vervormen, en zal de achtergebleven bel dus klein zijn; echter, voor kleine druppels en/of kleine inslagsnelheden werkt de oppervlaktespanning van de vloeistof de vervorming tegen, hetgeen het belvolume 
tevens verkleint. Tussen deze regimes in bevindt zich een overgangsgebied ('crossover'), alwaar het volume van de ingevangen bel maximaal is. Dit maximum wordt nauwkeurig bepaald door middel van schalingstheorie, de interferometrie-experimenten en de simulaties.

$\mathrm{Nu}$ we het mechanisme van invanging van kleine luchtbelletjes voor een druppelinslag op een solide oppervlak begrijpen, kunnen we ons afvragen of hetzelfde geldt voor de andere inslagscenario's: een bol op een vloeistofbad en een druppel op een vloeistofbad. Deze vraag staat centraal in Hoofdstuk 3. Gebruikmakend van computersimulaties en een verzameling van experimentele data uit de literatuur vinden we inderdaad dat het bovenstaande mechanisme universeel is en dat de grootte van de ingevangen bellen kwantitatief hetzelfde zijn voor de drie inslagscenario's, behalve dat de bellen voor de inslag van een druppel op een bad groter zijn met een factor 2. De equivalentie tussen de scenario's kunnen we begrijpen doordat de vervormingen verantwoordelijk voor de luchtbellen zeer gelokaliseerd zijn, typisch op een lengteschaal veel kleiner dan de grootte van de druppel of de bol, wat duidt op een symmetrie tussen de verschillende situaties. De factor 2 in het geval van druppelinslag op een bad kunnen we verklaren door het feit dat de gasdruk in dat geval 2 oppervlakken vervormt, in plaats van 1.

In Hoofdstukken 2 en 3 hebben we onze aandacht gericht op de uiteindelijke vervorming van de betrokken vloeistofoppervlakken, op het moment dat de twee oppervlakken elkaar raken. Deze bepaalt immers de grootte van de achtergelaten luchtbel. In Hoofdstuk 4 doen we een stap terug en passen we een 'verstoringsanalyse' toe om te berekenen hoe de eerste vervormingen groeien in de tijd en convergeren naar het uiteindelijke resultaat. Dit is van bijzonder belang omdat de schalingsargumenten in Hoofdstukken 2 en 3 hierop gebaseerd zijn. Deze vraag is minder makkelijk te beantwoorden dan het allicht lijkt, omdat we onderscheid moeten maken tussen inertiële en viskeuze luchtstroming, en tussen een grote en kleine luchtspleet (bepalend voor de geometrie van de stroming). We kwantificeren de twee overgangen in detail en we leiden de schalingsargumenten voor de verschillende regimes af. Voor 'typische' inslagwaarden (een druppel ter grootte van een millimeter die met de limietsnelheid (terminale snelheid) van een aantal meters per seconde naar beneden valt) vinden we dat het object eerst een fase met inertiële luchtstroming in een grote luchtspleet, vervolgens een fase met inertiële luchtstroming in een kleine luchtspleet, en tot slot een fase met viskeuze luchtstroming in een kleine luchtspleet ondergaat. Voor al deze fases leiden we de tijden ruimte-afhankelijke profielen van het vloeistofoppervlak af. We vinden, 
verrassend genoeg, dat zowel de inertiële als de viskeuze invloeden naar een uiteindelijke vervorming leiden in de orde van een micrometer. Echter, we concluderen nog steeds dat het belvolume voornamelijk bepaald wordt door de viscositeit van de lucht, aangezien de Stokes gasdruk sterk gelokaliseerd is en het meest sterk toeneemt gedurende de laatste fase alvorens de landing.

In Hoofdstuk 5 beschouwen we een trein van microdruppels die met hoge snelheid inslaat op een diep bad. De kracht van de herhaalde inslag is groot genoeg om een diepe en relatief smalle holte in het bad te creëren, met een diepte in de orde van duizend maal de grootte van de druppels zelf. De vorm van de holte en de bereikte diepte hangen voornamelijk af van een balans tussen massatraagheid van de vloeistof en oppervlaktespanning; door de kleine afmeting van de druppels heeft zwaartekracht pas een relevante invloed wanneer de holte diep genoeg is gegroeid. De ineenstorting van de holte wordt dus volledig bepaald door capillaire effecten, en vindt typisch plaats aan de bovenkant, bij het wateroppervlak. We identificeren de vorm van de holte, de tijd tot ineenstorting, en de maximale diepte.

In Hoofdstuk 6 maken we de stap van inslaande druppels naar zwevende druppels, oftewel druppels die volledig opgetild worden als resultaat van een continue gasstroming langs de onderkant van de druppel. Een typisch voorbeeld hiervan zijn Leidenfrost druppels, alwaar het gas waarop de druppel zweeft afkomstig is van de druppel zelf, die langzaam verdampt. Leidenfrost druppels kunnen spontaan beginnen te oscilleren en hun symmetrie verliezen; het mechanisme van deze instabiliteit, genoemd de 'ster-druppel-instabiliteit', is nog niet bekend. Door de gasstroming onder de druppel extern aan te drijven kunnen we de druppel laten zweven op een gaslaag op kamertemperatuur. In experimenten herkennen we in dit geval exact dezelfde druppeltrillingen als voor de Leidenfrost druppels, hetgeen laat zien dat de genoemde instabiliteit niet een gevolg is van temperatuurgradiënten of warmteoverdracht. De trillingen hebben dus een hydrodynamische achtergrond, en in Hoofdstuk 6 reproduceren we de instabiliteit in (hydrodynamische) computersimulaties. Binnen de simulaties zien we ook stabiele (dus niet trillende) zwevende druppels, of groeiende gasholtes die de druppels doen breken (de zogeheten 'schoorsteeninstabiliteit'). We vinden dat de aanzet van de druppeltrillingen uit twee fases bestaat: een axisymmetrische fase, die een samenspel is tussen de vloeistof- en de gasstroming, en (afhankelijk van de viscositeit) een fase waarin de trillende druppel symmetrie breekt met een voorname oscillatie modus, de Rayleigh frequentie. 
In Hoofdstuk 7 onderzoeken we hoe een druppel reageert op de inslag van een korte laserpuls. Dit werk is gemotiveerd door Extreem-ultraviolette (EUV) lithografie, een proces dat wordt toegepast voor de creatie van nanometerschaal patronen op elektronische chips. De rol van technologie in onze samenleving blijft groeien met een indrukwekkend hoog tempo. Voor het printen van deze patronen met hoge resolutie is straling met een extreem korte golflengte benodigd. In EUV-machines komt deze straling van tindruppels die worden beschoten door laserpulsen. Een eerste puls vervormt de druppel naar een platte vorm (sheet); namelijk, de sterke lokale verdamping als gevolg van de laserpuls resulteert in een terugslag van de druppel. De druppel transleert, vervormt en breekt op. Vervolgens ontvangt de tindruppel een tweede laserpuls, resulterend in de vorming van plasma. De vloeistofdynamica is cruciaal voor het verbeteren van het rendement van een EUV-machine. We passen experimentele analyse en computersimulaties toe, waarbij in het laatste geval de invoer van energie gemodelleerd wordt aan de hand van een zeer korte drukpuls werkend langs het druppeloppervlak. We vinden dat de snelheid en de maximale grootte van de aangeslagen druppel proportioneel zijn met de energie van de laserpuls. De expansie van de druppel wordt begrensd door oppervlaktespanning, een mechanisme gelijkend aan de begrenzing van de uitspreiding van een druppel die inslaat op een oppervlak.

Hoofdstuk 8 beschrijft de stromingen bij 'gieten', oftewel een (snelle) vloeistofstroom vanaf een gekromd solide oppervlak. Een typisch voorbeeld uit het dagelijks leven is de stroming van thee vanuit een theepot. Wanneer de stroomsnelheid van de thee te klein is, maakt de thee zich niet los van het oppervlak, maar blijft 'kleven', en loopt langs de tuit omlaag. Dit 'kleven' is een hydrodynamisch verschijnsel dat ook wel 'het theepot effect' genoemd wordt en op de zenuwen werkt van velen. Een eerder onderzoek heeft aangetoond dat de hoek waarmee de vloeistofstraal van het solide oppervlak separeert in onverwacht sterke mate afhangt van de hydrofobiciteit van het oppervlak (en dat het theepot effect zelfs volledig voorkomen kan worden door een hydrofoob oppervlak te gebruiken). Een fysische verklaring voor het 'kleven' zelf was echter nog niet gevonden. Door de wet van impulsbehoud op te lossen, meenemende het precieze stromingsprofiel, de geometrie van het probleem, en de capillaire krachten, vinden we analytisch dat, inderdaad, er voor kleine stroomsnelheden geen stabiele stroming mogelijk is. We hebben deze minimale stroomsnelheid gekwantificeerd, als een functie van de relevante parameters, en vinden kwalitatieve overeenstemming met de belangrijkste experimentele resultaten. 


\section{List of scientific articles}

Drop shaping by laser-pulse impact

A.L. Klein, W. Bouwhuis, C.W. Visser, H. Lhuissier, C. Sun, J.H. Snoeijer, E. Villermaux, D. Lohse, H. Gelderblom

Physical Review Applied 3, 044018 (2015)

Initial surface deformations during impact on a liquid pool W. Bouwhuis, M.H.W. Hendrix, D. van der Meer, J.H. Snoeijer Journal of Fluid Mechanics 771, 503-519 (2015)

Oscillating and star-shaped drops levitated by an airflow W. Bouwhuis, K.G. Winkels, I.R. Peters, P. Brunet, D. van der Meer, J.H. Snoeijer

Physical Review E 88, 023017 (2013)

Erosion evolution in mono-crystalline silicon surfaces caused by acoustic cavitation bubbles

D.F. Rivas, J. Betjes, B. Verhaagen, W. Bouwhuis, T.C. Bor, D. Lohse, H. Gardeniers

Journal of Applied Physics 113, 064902 (2013)

Maximal air bubble entrainment at liquid drop impact

W. Bouwhuis, R.C.A. van der Veen, T. Tran, D.L. Keij, K.G. Winkels,

I.R. Peters, D. van der Meer, C. Sun, J.H. Snoeijer, D. Lohse

Physical Review Letters 109, 264501 (2012)

Laser impact on a drop

A.L. Klein, C.W. Visser, W. Bouwhuis, H. Lhuissier, C. Sun, J.H. Snoeijer, E. Villermaux, D. Lohse, H. Gelderblom Award winning video entry Gallery of Fluid Motion 2014

To appear in Phys. Fluids, 2015 
Universal mechanism for air entrainment during liquid impact M.H.W. Hendrix, W. Bouwhuis, D. van der Meer, D. Lohse, J.H. Snoeijer Submitted to Journal of Fluid Mechanics

Effect of wettability on inertial pouring flows

W. Bouwhuis, J.H. Snoeijer

Submitted to Physical Review E

Impact of a high-speed train of microdrops on a liquid pool

W. Bouwhuis, X. Huang, C.U. Chan, P.E. Frommhold, C.D. Ohl, D. Lohse, J.H. Snoeijer, D. van der Meer

In preparation

Drop deformation by laser-pulse impact

H. Gelderblom, H. Lhuissier, A.L. Klein, W. Bouwhuis, D. Lohse,

E. Villermaux, J.H. Snoeijer

In preparation

Droplet impact on a liquid pool and bubble entrainment for low Bond numbers

C.P. Sleutel, P.-H. Tsai, W. Bouwhuis, M.-J. Thoraval, C.W. Visser, A. Wang, M. Versluis, D. Lohse

In preparation 


\section{Acknowledgements}

The five years during which I participated in the research of the Physics of Fluids group (being the second half of my 10 years lasting journey at the University of Twente) have been fantastic. I was amazingly lucky to be surrounded by a large bunch of colleagues with a splendid enthusiasm for their own and other people's study, and very helpful family and friends. Without them, the studies presented in this thesis would not have been at this stage/level (probably would not exist at all), and, moreover, these five years would definitely not have been so pleasurable. I would like to thank all of these people, although it will be impossible to mention all of them who (and institutions which) contributed to the results presented in this thesis and the fun in the period September 2010 - August 2015.

I want to express my deepest gratitude to my advisers Jacco Snoeijer, Devaraj van der Meer, and Detlef Lohse, who gave me the opportunity to take part in their leading research.

Jacco, I do not think anyone could wish for a better supervisor and promotor than you have been during my master- and PhD-trajectory. Your enthusiasm is catching, which is only one of the reasons I experienced our 'weekly' meetings as very pleasant. Your insight in physical problems is amazing, and even more amazing is your ability to explain the strategies to solve these problems very clearly and inspiring. Moreover, you are a very good 'mentor' who always cares about someone's feelings about his or her work. I think all of your students/coworkers regard you as a real friend.

Devaraj, I am also very lucky to have collaborated with you during the past 5 years, in particular when it came to the broad spectrum of impact phenomena, or to the many details of the Boundary Integral (BI) code. It was exciting to experience again and again how much you know about the properties and possibilities of this (and other) simulation code(s). Your broad knowledge of fluid physics and also other topics in physics that we discussed, together with your great enthusiasm in sharing this knowledge with students and coworkers, has been very helpful. I am glad to have you as a second promotor. 
Detlef, I would like to thank you for your magnificent insight of which paths we needed to take in our research. You always had a perfect overview and vision on what parts of our work needed extension, which resulted in useful collaborations and nice publications. Also because of this, it was really a privilege to do research and collaborate with colleagues working in the Physics of Fluids group.

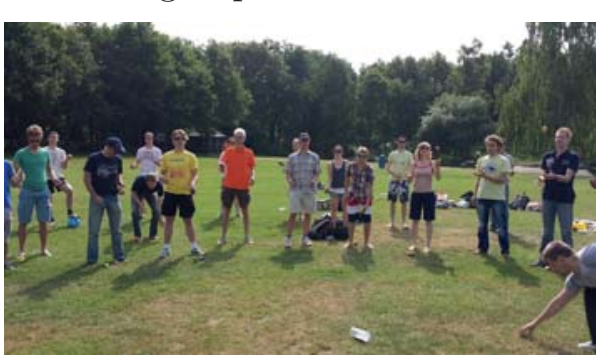

Juggling workshop during the Physics of Fluids group outing, Het Hulsbeek, Oldenzaal, the Netherlands, July 2013

I would like to stress that, besides Jacco, Devaraj, and Detlef, the other staff members were also able to convey their enthusiasm to me (and to my colleagues), of whom I in particular would like to mention Chao Sun and Leen van Wijngaarden. Chao, I really enjoyed our collaboration on impact phenomena, and I also want to thank you for making time to teach me and a few of my fellow students experimental techniques in physics of fluids (together with Yoshi Tagawa) - out of the regular class times. Leen, we once spent a full afternoon on brainstorming on possible mechanisms causing the fascinating large bubble entrapment phenomenon at liquid-drop impact. The reason that this took a full afternoon was the enormous number of inspiring suggestions that you provided on this subject. Surprisingly, the only parameter which I immediately excluded from having any influence on large bubble entrapment, the viscosity of the liquid, appeared to be the 'driving' parameter behind the mechanism later on. This was shown by the revealing GERRIS simulations run by my colleague Marie-Jean Thoraval. 
I want to thank Ivo Peters for his guidance on the BI code, which has become the key ingredient of all the chapters in this thesis. Ivo, this would not have been possible without your availability to discuss the several numerical stages we have passed, which actually continued until the moment that this thesis is in press.

Next I want to thank Maurice Hendrix. I supervised Maurice during his graduation assignment, but I experienced this period as a fruitful collaboration period, rather than as a

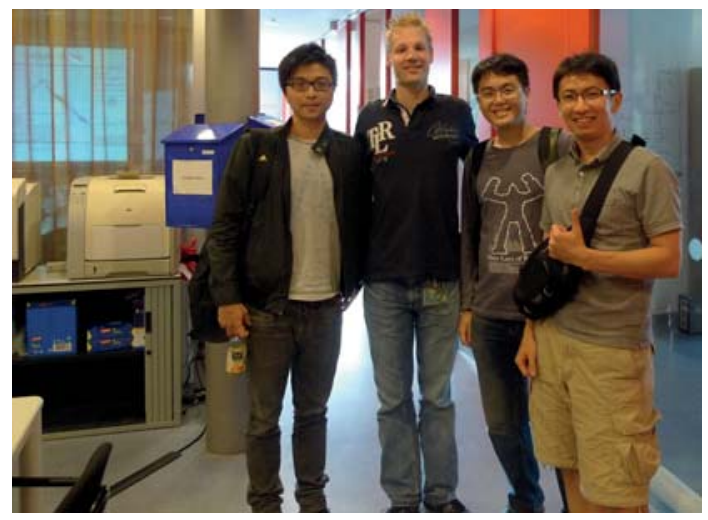

Several visitors have learned about impact and the Boundary Integral simulation code. On the background the 'maximum bubble entrapment' plot, which has become the key ingredient of this thesis.

'teaching' period. Basically, Maurice received the BI code, he left the Netherlands for a month, and returned with a working modified code, which was an important goal of his assignment. Maurice, your vision, independence, and motivation are fantastic, resulting in the fact that I might have learned as many from you, or more, as the other way around. You have done (and are still doing) an excellent job, which has been of great importance for this thesis.

I also want to thank the second master student I supervised, Akihito Kiyama, from Tokyo University. Akihito did an outstanding internship in our group; in only (about) 10 weeks, he showed to be able to learn the BI code and build a new setup, answered the research questions he proposed beforehand in detail, and even invited for doing nice future collaboration using the code. Aki, I really enjoyed working with you. 


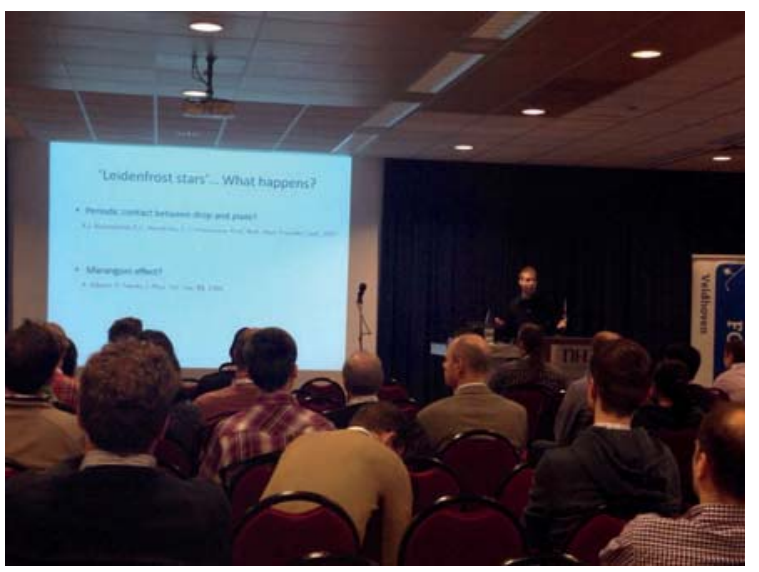

Giving a conference talk on star drops during the yearly FOM meeting at Veldhoven, the Netherlands, January 2014.
The list of people with whom I had the opportunity to cooperate on several projects is enormous. It is obvious that without them, the presented results in this thesis, being just a summary of all the output from the cooperations, would not have been there. In addition to the already mentioned people, I thank Roeland van der Veen and Tuan Tran for their experiments on drop impact, Koen Winkels for his work on levitated drops, initial deformations of drops or surfaces during impact, and the entrapped air bubble size predictions (together with Diederik Keij), and Hanneke Gelderblom, Alexander Klein, Marise Gielen, and Emmanuel Villermaux for the fruitful cooperation on deforming drops impacted by a laser pulse. Also many thanks to Philippe Brunet (levitated drops), David Fernandez Rivas and Bram Verhaagen (erosion by cavitation bubbles), Claas-Willem Visser, Peichun Amy Tsai, Francesco Fornarelli, Paolo Oresta, Pei-Hsun Tsai, MarieJean Thoraval, Pascal Sleutel, Michiel van Limbeek, Minori Shirota, Erik-Jan Staat, Yaxing Li, Sander Wildeman, Rodrigo Ezeta and Mahening Citravidya (drop impact), Xin Huang, Chon U Chan, Philipp Frommhold and ClausDieter Ohl (drop train impact), Tak Shing Chan (inertial contact lines), and Henri Lhuissier (liquid bells). A special gratitude to Pascal Sleutel for being an 'epic' lab teacher and coworker. Pascal, your dedication to your work is 'royal'! Arjan Fraters will take over the BI code from me. Arjan, I enjoyed working with you and you will be a great successor of the code! I am grateful to Christophe Clanet, Stephen Wilson, Andrea Prosperetti, James Sprittles, Michel Riepen (ASML), Herman Wijshoff (OCÉ), Hans Reinten (OCÉ), Antonin Eddi, and Stefan Karpitschka for the discussions/suggestions on our work. 


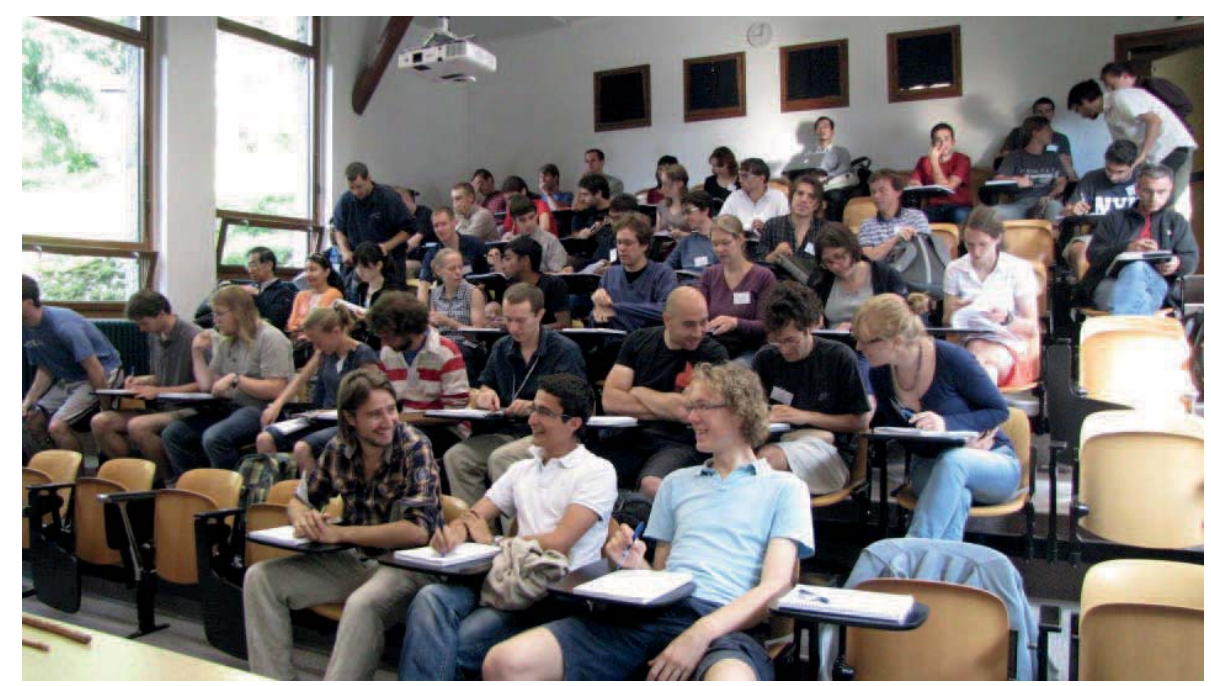

Entertained and entertaining colleagues at the Soft Interfaces Summer School at Les Houches, France, July 2012.

It was a great pleasure to share an office with Bram Verhaagen, Rajaram Lakkaraju, Erik-Jan Staat, Peter van Dijk, Maarten Kok, Adeline Pons, Theo Driessen, and Arjan Fraters. Office colleagues seem to be collaborators automatically and I do not think there is anyone who has (nolens volens) experienced this more than Peter... Peter, it was really a great pleasure to discuss with you on

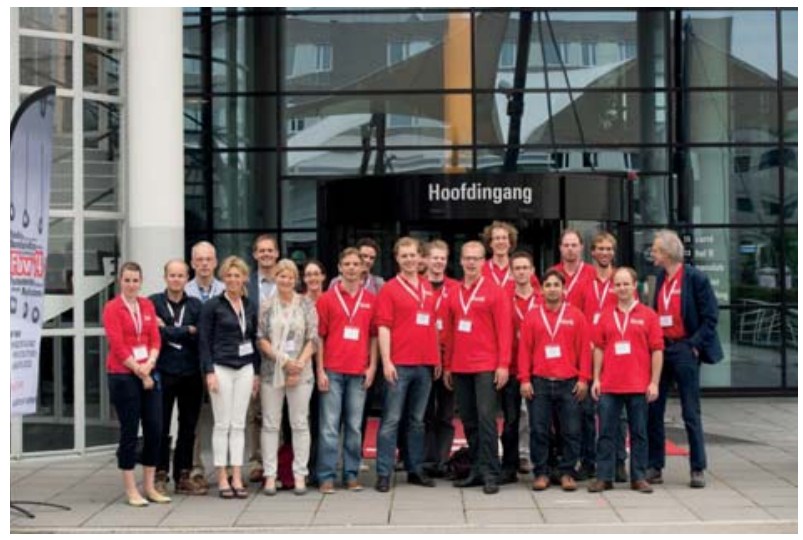

The 'Red Brigade', the local organizing committee of the wonderful Flow14 conference, University of Twente, the Netherlands, May 2014. physical and/or numerical phenomena/limitations, and all of your suggestions and comments were really helpful and inspiring! This also holds for your great advice on many English language issues. 
Thanks to Edip Can and Rory Dijkink (Saxion Hogeschool Enschede) for their willingness to share their experiences of being a 'university of applied sciences' teacher/researcher and giving me good advice. Thanks to Joke Oosterhuis and Cornelise Vreman (Onderwijskundige Dienst University of Twente) for the very useful workshops and meetings on teaching skills.

I had great fun taking part in the 'Fysica van Vloeistoffen' course team with Joost Weijs, Roeland van der Veen, Rianne de Jong, Jacco Snoeijer, and Devaraj van der Meer. It is not only because of the enthusiastic students, but also because of this magnificent team that I really enjoyed the teaching tasks. The same applies to the 'Dynamica' and 'Arago Ouderdag' course team; Bijoyendra Bera, Aram Klaassen, and Michel Duits.

Thanks to Joanita Leferink for her contribution to my PhD trajectory; Joanita seems to be the only group member who knows an (efficient) solution to every problem, which is actually a very good scientific skill... Thanks to Bas Benschop for frequently fixing computer problems, in particular the inevitable Kefalotiri-connection-, MAPLE-license-, and Windows-MAC-conversion-related issues. As one of the few non-experimenters in the group (until the final half year of my $\mathrm{PhD}$ ), I did not have the privilege to spend much time together with Gert-Wim Bruggert and Martin Bos, but I really appreciated their role as the technicians to whom I could ask everything.
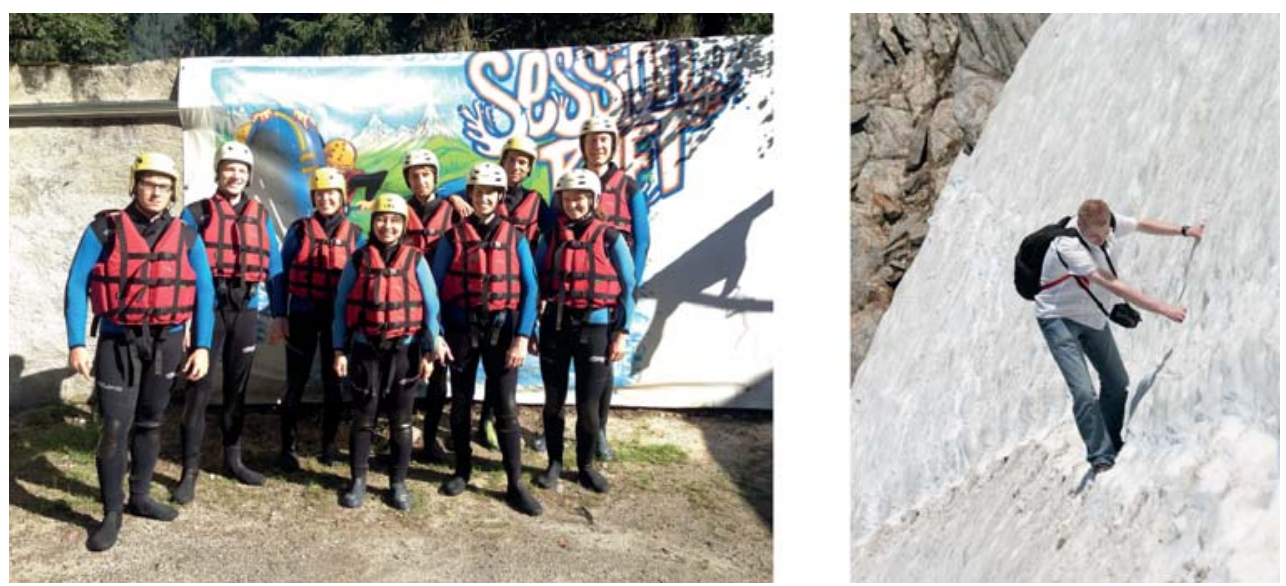

The tough Summer School at Les Houches, France, July 2012. 
I am grateful to all PoF-group (and Flow14-conference) colleagues for the nice conversations and for all the fun and excitement. Many thanks to Roeland van der Veen, Tuan Tran, Maarten Kok, Erik-Jan Staat, Sander Huisman, Tim Segers, and Guillaume Lajoinie for sharing their experience on making nice figures, nice movies, and nice posters.

I would never got where I am today without the unconditional support of my family throughout my entire study and PhD-trajectory in Twente. My parents Dick and Ilse have always been there for me. This means a lot to me. The same applies for my sister Suzan, and Floris, who nowadays also experience the beauty of Enschede. Oma Meulink, oma Bouwhuis, oom Jan Hendrik, tante Jannie, oom Wim, tante Tineke, Leonie, Erjan, Marieke, Simon, Kirsten, Richard, Maureen, Michiel, Matthijs, Vivian, Norah, Floor, Loes, Davida, Pascal, Kevin, Bart, Dennis, Bastiaan, Maarten, Delta FM and Glazen-Café DJs and colleagues (in particular the colleagues of 'De Delta FM Weekend Show' at Friday nights), former supermarket Veld Vroomshoop colleagues, former fellow villagers from 'The Diamond Of Twenterand' Vroomshoop, Paygo, Mellow, Sonny \& Cher, Smurf and Otto... and all my other friends and people I met so far..., thanks for the fun times, and for showing interest always (and even sometimes when I talked about my work! ;)).

Suzan and Leonie, my paranimphs, a special word for you, for giving very important input being mental supporters during this special ceremony in my life. Please know that 'during the ceremony' is really an understatement, because you have played this supporting role during my full $\mathrm{PhD}$ trajectory, and longer.

Wilco Bouwhuis Enschede, July 212015 



\section{About the author}

Wilco Bouwhuis was born on the first of May 1987 in Vroomshoop, Overijssel, The Netherlands. He graduated

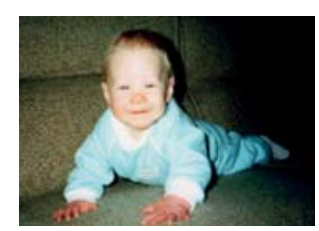
from high school (CSG Het Noordik, Almelo) in 2004, after which he studied one year at Saxion Hogeschool Enschede (2004-2005) and obtained the Propedeuse Media Engineering (Electrical Engineering), to develop a beautiful hobby at the local radio \& TV station Delta FM: presenting, taking care of the technical realization of several radio programs, and guiding new employees.

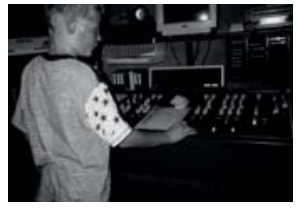

1997

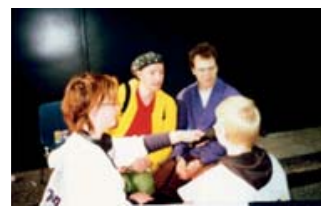

2000

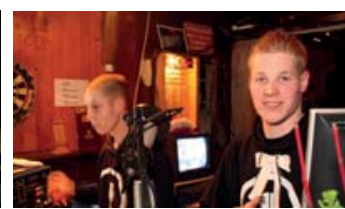

2008

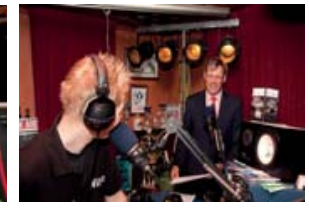

2010

Theoretical physics and mathematics were only a small portion of this study program, but he had so much fun with it that he decided to switch to the University of Twente to study Applied Physics. He obtained his Bachelor's degree in 2008 at the Physics of Complex Fluids group, with the thesis 'Improving immersion lithography using electrowetting'. In 2010 he obtained his Master's degree in Applied Mathematics in the Numerical Analysis and Computational Mechanics group at the University of

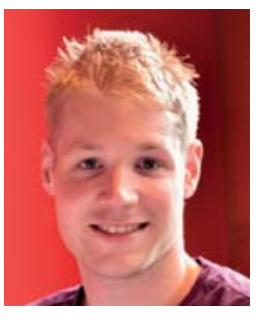
Twente, for his thesis 'Modeling the Neocortex: Large scale simulation \& analysis'. In the same year he joined the Physics of Fluids group at the University of Twente, to receive a second Master's degree in Applied Physics (2011). This is where he started his PhD project in September 2011 with professor Jacco Snoeijer, professor Devaraj van der Meer, and professor Detlef Lohse. 



\section{Stellingen \\ behorende bij het proefschrift \\ Dynamics of Deforming Drops}

Wilco Bouwhuis, 28 augustus 2015

1. De hoeveelheid lucht die wordt ingevangen in een bel tijdens de inslag van een druppel op een solide ondergrond, ten gevolge van de viskeuze weerstand geleverd door de tussenliggende luchtlaag, kent een maximum voor een bepaalde inslagsnelheid.

Hoofdstuk 2 van dit proefschrift

2. Het mechanisme voor de insluiting van een luchtbelletje als gevolg van de omringende lucht is hetzelfde voor de inslag van een druppel op een solide ondergrond, de inslag van een solide bol op een bad, en de inslag van een druppel op een bad.

Hoofdstuk 3 van dit proefschrift

3. De spontane trilling en symmetriebreking van Leidenfrost druppels hebben een hydrodynamische achtergrond en worden dus niet geïnduceerd door een eventueel temperatuurverschil binnen de druppel, of door de verdamping van de druppel.

Hoofdstuk 6 van dit proefschrift

4. Bij het theeschenken vindt het vervelende 'plakken' van de thee tegen de tuit van de theepot plaats bij een stroomsnelheid waarvoor er geen oplossingen bestaan voor een stabiele straal separerende vanaf de tuit. Dit gebeurt wanneer de gietsnelheid te klein is, de tuit te stomp is, of de tuit te hydrofiel is.

Hoofdstuk 8 van dit proefschrift

5. Het gebruik van een simulatiecode die een zuivere potentiaalstromingsvloeistof simuleert, met als doel het vinden van stabiele, niet in tijd veranderende, oplossingen, is dikwijls een slecht idee.

6. De kwaliteit van het uitleggen, opslaan en doceren van onderzoek is minstens even belangrijk als de kwaliteit van het onderzoek zelf.

7. Wetenschap is niet uit te drukken in werkuren. Isaac Newton was met het tegenovergestelde bezig van wat veel mensen definiëren als 'werkuren maken', toen, naar hoe de anekdote luidt, de vallende appel op zijn hoofd terecht kwam, met zijn baanbrekende gedachte betreffende zwaartekracht als gevolg. Ook in de tegenwoordige tijd lijken de beste wetenschappelijke ideeën eerder te ontstaan onder de douche of op de fiets dan achter het bureau. Les Houches in Frankrijk, gelegen aan de voet van de Mont Blanc, is dus een voorbeeld van een ultiem efficiënte locatie voor een fysische zomer- (of winter-) opleiding. Binnen dezelfde redenering moet het gebruik van Facebook door promovendi tijdens hun 'werkuren' toegestaan blijven. 


\section{Propositions}

accompanying the thesis

\section{Dynamics of Deforming Drops}

Wilco Bouwhuis, August 282015

1. The amount of air entrapped into a bubble during the impact of a liquid drop on a solid surface, caused by the viscous resistance delivered by the air-layer in between the drop and the surface, has a maximum for a certain impact velocity.

Chapter 2 of this thesis

2. The mechanism for the entrapment of a tiny air bubble due to the effect of the surrounding air is identical for drop impact on a solid surface, sphere impact on a pool, and drop impact on a pool.

Chapter 3 of this thesis

3. The spontaneous oscillation and symmetry breaking of Leidenfrost drops have a hydrodynamic origin, and, thus, are not induced by any temperature gradient within the drop, or by the evaporation of the drop.

Chapter 6 of this thesis

4. During the pouring of tea, the annoying 'sticking' of the tea against the spout of the teapot occurs at stream velocities at which there exists no physical solution for a steady separating jet. This is the case for too small pouring velocities, or for teapot spouts which are too blunt or too hydrophilic.

Chapter 8 of this thesis

5. The use of a numerical simulation code which assumes and simulates a pure potential flow liquid for finding stable, steady solutions, is often a bad idea.

6. The quality of explaining, reporting, and teaching research is at least as important as the quality of the research itself.

7. Science is not expressible in working hours. Sir Isaac Newton was doing the opposite of what many people define as 'working', when, to how the story reads, the falling apple landed on his head, which resulted in Newton's groundbreaking idea about gravity. Also in the present tense, the best scientific ideas seem to arise during cycling or taking a shower, rather than during sitting behind a desk. Les Houches in France, located at the foot of the Mont Blanc, is thus an example of an ultimately efficient location for a summer (or winter) school in physics. Within the same reasoning, using Facebook during 'working hours' should remain authorized for PhD students. 